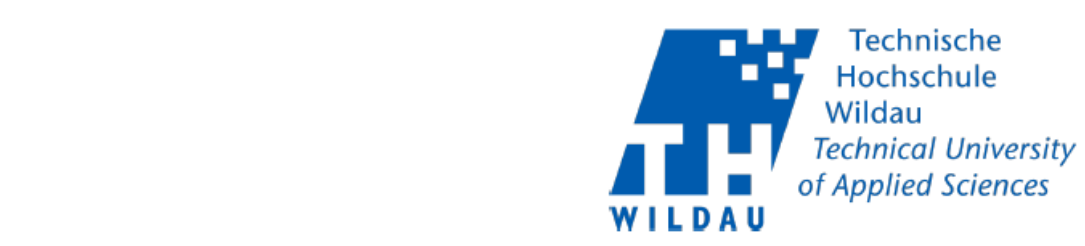

Masterarbeit

zur Erlangung des akademischen Grades

Master

Technische Hochschule Wildau

Fachbereich Wirtschaft, Informatik, Recht

Studiengang Bibliotheksinformatik

Thema (deutsch): Konzeption und prototypische Umsetzung eines mit standortbasierten Informationen arbeitenden Systems für die Nutzer des Philologicums der Ludwig-Maximilians-Universität München.

Thema (englisch): Conception and prototypical implementation of a system working with location-based information for the users of the Philologicum of the Ludwig-Maximilians-University Munich.

Autor/in: Kai Barth

Matrikel-Nr.: $\quad 50041583$

Seminargruppe: $\quad B I M / 16$

Betreuer/in: $\quad$ Prof. Dr. rer. nat. Janett Mohnke

Zweitgutachter/in: Dr. Frank Seeliger

Eingereicht am: $\quad 18.01 .2019$ 


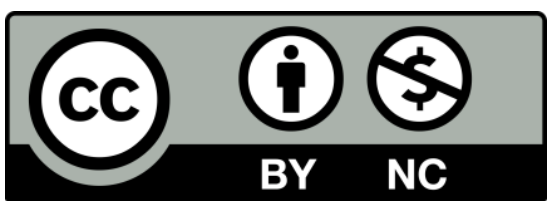

\section{Kai Barth}

Konzeption und prototypische Umsetzung eines mit standortbasierten Informationen arbeitenden Systems für die Nutzer des Philologicums der Ludwig-Maximilians-Universität München

Masterarbeit, 18.01.2019

Gutachter: Prof. Dr. rer. nat. Janett Mohnke und Dr. Frank Seeliger

Wildau Institute of Technology

Technische Hochschule Wildau

Hochschulring 1

15745 Wildau 


\section{Danksagung}

Ich möchte zunächst all jenen danken, die durch ihre fachliche und persönlichen Unterstützung zum Gelingen dieser Masterarbeit beigetragen haben.

An erster Stelle möchte ich Herrn Weiß nicht nur für die Bereitstellung des interessanten Themas, sondern ebenso für die stets tatkräftige Hilfe danken. Darüber hinaus danke ich ebenfalls Frau Berg für Ihre Unterstützung und gute Zusammenarbeit während zahlreichen Korrespondenzen und Konsultationen.

Mein Dank gilt gleichermaßen Frau Prof. Dr. Mohnke für die Begleitung und freundliche Hilfsbereitschaft während meiner Masterarbeit.

Mein besonderer Dank gilt meiner Freundin, die mich während der gesamten Zeit hervorragend unterstützt hat. Vor allem danke ich dir für deine aufmunternden Worte und deine engelsgleiche Geduld. Ohne dich wäre diese Arbeit nicht entstanden! 


\section{Abstract}

Ziel der Masterarbeit ist es ein, mit standortbasierten Informationen arbeitendes System für die Nutzer des Philologicums der Ludwig-Maximilians-Universität München zu konzipieren und prototypisch umzusetzen. Fundament eines solchen Systems muss eine valide Positionsbestimmung unter Beachtung der gegebenen Rahmenbedingungen sein. Neben der Vermittlung theoretischer Grundlagen, die das Thema „Indoor Positioning System" tangieren, ist ein wesentlicher Schwerpunkt der Arbeit den aktuellen Stand der Technik, im Hinblick auf die Positionsbestimmung in Innenräumen zu eruieren.

In einer kaskadierenden und durch praktische Analysen untermauerten Evaluation verschiedener Positionierungstechnologien ist schließlich Optical Character Recognition, als Mittel der Wahl bestimmt worden. Basierend auf den Ergebnissen der Anforderungs- und Zielgruppenanalyse sowie den gewonnen Erkenntnissen zu „Locationbased Services“ ist ein, in der Form im Bibliothekswesen neuartiges System konzipiert und entwickelt worden.

Die im Rahmen der prototypischen Umsetzung entstandenen Komponenten demonstrieren anschaulich die Funktionsweise und Machbarkeit eines Systems, dass mit standortbasierten Informationen arbeitet. Kernelement ist zum einen die Webanwendung "LMU-Linker" als Middleware, im Sinne eines verknüpfenden Bindeglieds zwischen Point of Interests und dazugehörigen Inhalten. Zum anderen ist das Frontend, die auf Android basierte App „Philologicum“, zur Bestimmung des Standortes und zur Anzeige der verknüpften Inhalte, essentieller Bestandteil des Prototyps.

Die Masterarbeit soll als Auftragsarbeit die Grundlage für den zukünftigen Produktivbetrieb des Systems, im entstehenden Philologicum, bilden. Die gewonnen Erkenntnisse können jedoch gleichermaßen auch für potentiell Interessierte, mit Fokus auf den Themenbereichen „Indoor Positioning“ und „Location-based Services“, aufschlussreich sein. 


\section{Abstract (engl.)}

The aim of the master thesis is to design a system working with location-based information for the users of the Philologicum of the Ludwig-Maximilians-University Munich and to implement it prototypically. The foundation of such a system must be a valid positioning under consideration of the given conditions. In addition to imparting further theoretical basics concerning the topic "indoor positioning system", an essential focus of the work is to determine the current state of the art with regard to positioning in indoor spaces.

In a cascading evaluation of different positioning technologies supported by practical analyses, optical character recognition was finally determined as the means of choice. Based on the results of the requirements and target group analysis as well as the findings on "location-based services", an innovative system has been conceived and developed.

The components developed within the prototypically implementation clearly demonstrate the functionality and feasibility of a system that works with location-based information. Core element is on the one hand the web-application "LMU-Linker" as a middleware in the sense of a connecting link between point of interests and related content. On the other hand, the frontend, the Android-based app "Philologicum", is an essential component of the prototype for determining the location and displaying linked contents.

The master thesis as a commissioned work should form the basis for the future productive operation of the system in the emerging Philologicum. However, the insights gained can also be informative for potentially interested parties, with a focus on the subject areas "indoor positioning" and "location-based services". 


\section{Hinweise zum Lesen der Arbeit}

Die in dieser Arbeit verwendeten Fachbegriffe werden in einem alphabetischen sortierten Glossar am Ende der Arbeit erläutert. Die Anordnung am Schluss soll der Verbesserung des Leseflusses dienen und dem Leser die eigenständige Entscheidungsmöglichkeit bieten weiterführende Informationen zu den Fachtermini nachzuschlagen. Begriffe, die im Glossar erklärt werden sind mit nach rechts und links zeigenden doppelten spitze Anführungszeichen umschlossen und eindeutig gekennzeichnet. (Beispiel: "Glossar«). Darüber hinaus sind die Fachtermini in der PDF-Version mit dem jeweiligen Glossareintrag verlinkt.

Abkürzungen werden bei erstmaligen Auftauchen ausgeschrieben und die danach verwendete Abkürzung in Klammern gesetzt. Taucht die Abkürzung im weiteren Verlauf des Textes erneut auf wird diese nicht noch einmal gesondert gekennzeichnet. Abkürzungen, die im Glossar erläutert werden sind auch im Abkürzungsverzeichnis mit den doppelten spitzen Anführungszeichen markiert.

Zitate im Text werden durch Quellen in Kurzform mit Seitenangabe gekennzeichnet und sind für den besseren Lesefluss in Fußnoten ausgelagert. Alle verwendeten Quellen werden ausführlich im alphabetisch sortierten Literaturverzeichnis aufgelistet.

Für Abbildungen und Tabellen gibt es je ein separates Tabellen- und Abbildungsverzeichnis am Schluss der Arbeit. Bei Abbildungen wird zusätzlich eine Herkunftsangabe über Fußnoten an der Bildunterschrift kenntlich gemacht. Da sämtliche Tabellen auf eigener Datenerhebung beruhen ist auf eine Herkunftsangabe, zugunsten der Lesbarkeit verzichtet worden.

Während der Bearbeitung der vorliegenden Arbeit sind zahlreiche Daten, in Form von Messungen, Analysen, Quellcode, Videos, usw. entstanden. Aufgrund des Umfanges hat sich der Autor entschieden den Anhang nur digital zur Verfügung zu stellen. In den Fußnoten findet sich die relative Pfadangabe, gekennzeichnet durch ein „Pfad:““. In der PDF-Version sind Verlinkungen zum Anhang gesetzt, sodass der Leser die entsprechenden Ordner bzw. Dateien direkt beim Rezipieren der Arbeit aufrufen kann. Dazu ist es wichtig, dass im Verzeichnis des PDF's der Ordner „Anhang“ vorhanden ist. Dieser Ordner sowie der Quellcode für sämtliche Anwendungen sind auf der beiliegenden $C D$ verfügbar. 
Des Weiteren ist der Quellcode über das GitHub-Repository des Autors zugänglich. Das Repository ist unter der URL https://github.com/KaiBarth/Masterarbeit erreichbar und enthält neben der Masterarbeit sämtliche Anhänge und Daten. Über die Funktion "Clone or download“" können alle Inhalte komfortabel erreicht werden. Es ist anzumerken, dass der aktuelle Entwicklungsstand, aufgrund des Voranschreiten des Projektes von der im Rahmen der Arbeit veröffentlichten Version abweicht. 


\section{Abkürzungsverzeichnis}

AOA Angle of Arrival

AP Access Point

API Application Programming Interface

AJAX Asynchronous JavaScript and XML«

BLE Bluetooth Low Energy

BT Bluetooth

CMS Content Management System

CSS Cascading Stylesheet

GPS Global Positioning System

GUI Graphical User Interface

ID Identifier

IPS Indoor Positioning System

JSON 》JavaScript Object Notation«

LBS Location-based Service

LMU Ludwig-Maximilians-Universität München

NFC Near Field Communication

OCR Optical Character Recognition

OPS Outdoor Positioning System

OS Operating System

PHP PHP: Hypertext Preprocessor

POI Point of Interest

RFID Radio-Frequency Identification

RSSI Received Signal Strength Indication

SQL Structured Query Language

TDOA Time Difference of Arrival

TOA Time of Arrival

UB Universitätsbibliothek

UI User Interface

UID Unique Identifier

WLAN Wireless Local Area Network

XML Extensible Markup Language 


\section{Inhaltsverzeichnis}

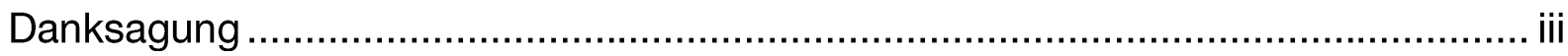

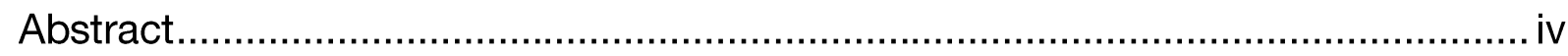

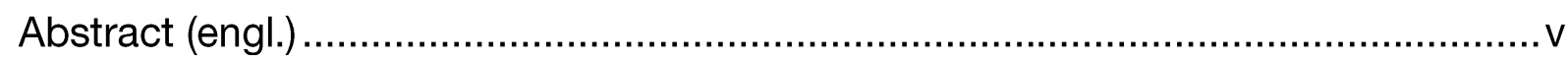

Hinweise zum Lesen der Arbeit........................................................................ vi

Abkürzungsverzeichnis ................................................................................. viii

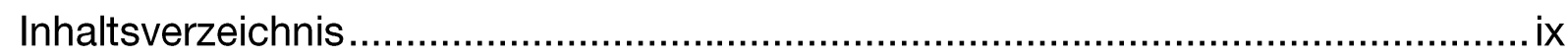

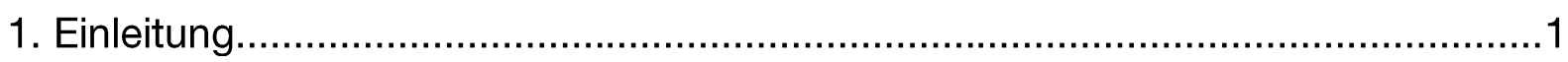

2. Der Neubau des Philologicums der Universitätsbibliothek ...................................4

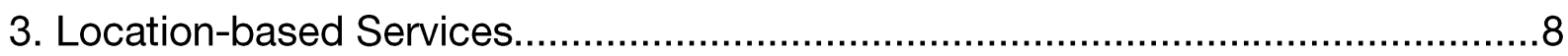

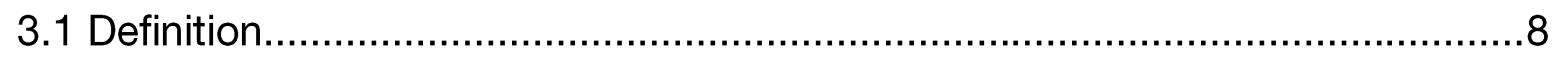

3.2 Architektur von Location-based Services...................................................10

3.3 Ausgewählte Location-based Services in Bibliotheken.................................14

4. Das Smartphone als Frontend für Location-based Services................................21

4.1 Überblick Smartphonemarkt................................................................21

4.2 Betriebssysteme von Smartphones........................................................22

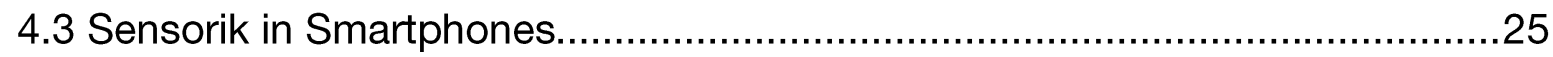

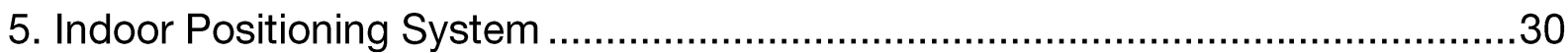

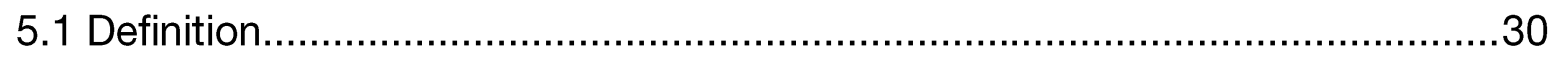

5.2 Nicht-funktionale Anforderungen..............................................................

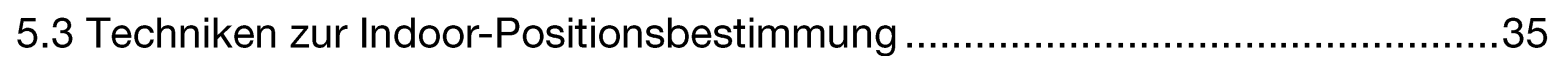

5.3.1 Positionsbestimmung anhand von Signaleigenschaften..........................36

5.3.2 Algorithmen zur Positionsbestimmung ………………………….........38

5.4 Technologien zur indoor Positionsbestimmung............................................

5.4.1 Positionierung auf Basis von Infrarot ...................................................

5.4.2 Positionierung auf Basis von Ultraschall \& hörbaren Tönen ......................43 
5.4.3 Positionierung auf Basis von Magnetismus

5.4.4 Positionierung auf Basis von optischen \& sichtbasierten Technologien ...45

5.4.5 Positionierung auf Basis von Funkfrequenzen .......................................47

5.4.6 Positionierung auf Basis von sichtbaren Licht .....................................51

5.4.7 Positionierung auf Basis von Koppelnavigation ......................................52

6. Anforderungs- und Zielgruppenanalyse .......................................................54

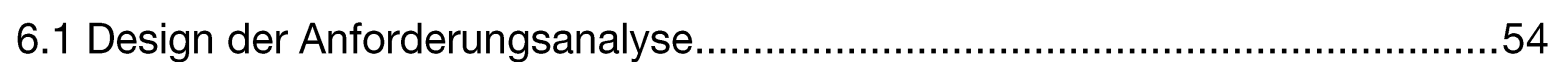

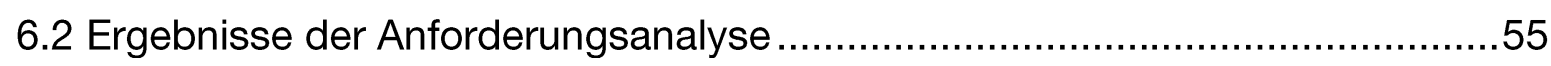

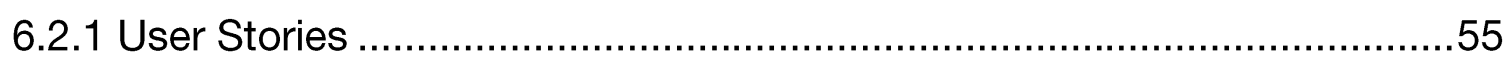

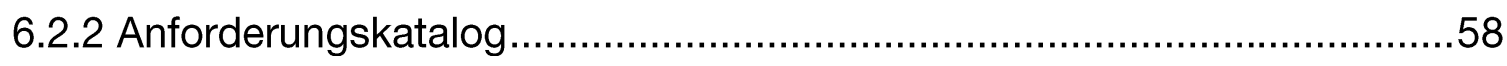

6.2.3 Sonstige zu beachtende Rahmenbindungen ........................................61

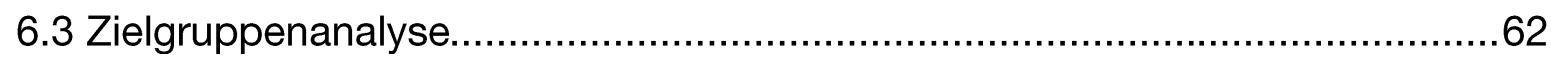

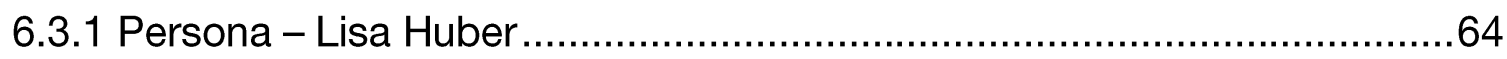

6.3.2 Persona - Krista Vogeler ..................................................................6

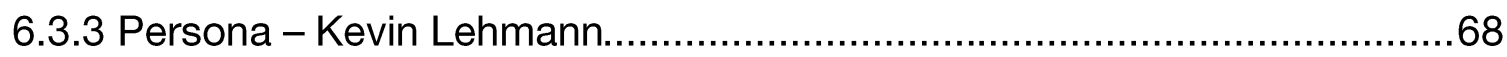

6.3.4 Persona - Louis Momodou ....................................................................69

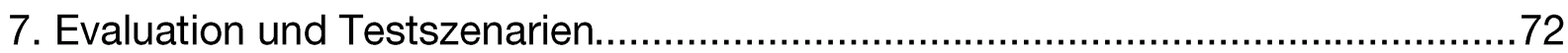

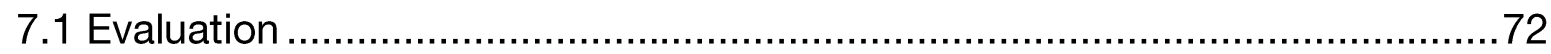

7.1.1 Evaluationsstufe 1 - Sensorik und Zukunftsfähigkeit ................................72

7.1.2 Evaluationsstufe 2 - zusätzliche technische Infrastruktur und Kosten ..........73

7.1.3 Evaluationsstufe 3 - Initialisierungs- und Wartungsaufwand........................74

7.1.4 Evaluationsstufe 4 - Anfälligkeit für Störfaktoren und Abhängigkeit von

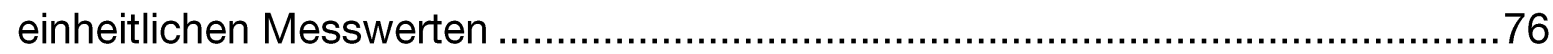

7.2 Evaluation ausgewählter Technologien mittels praktischer Testszenarien ......79

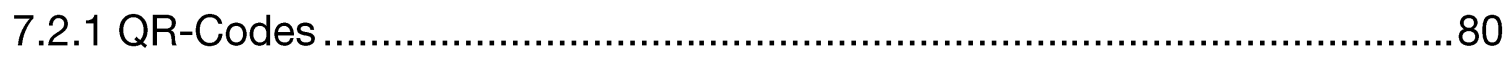

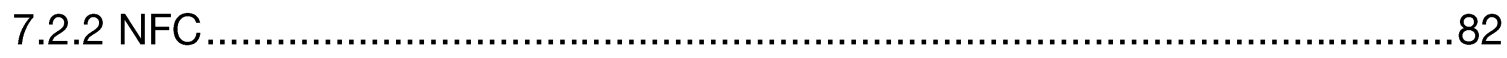

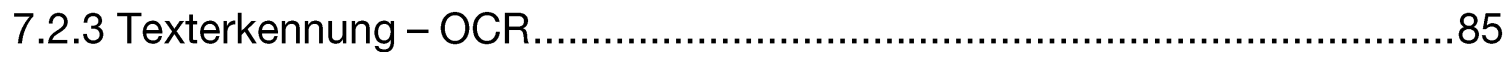


7.3 Ergebnis Evaluation und praktische Tests.................................................90

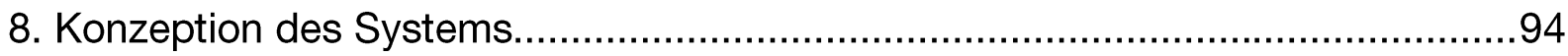

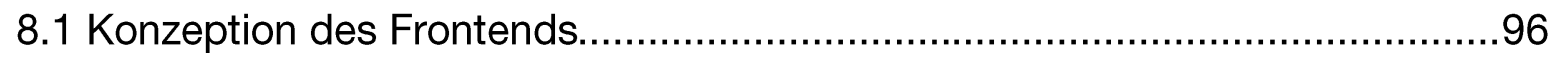

8.1.1 Frontend GUI - Gesamtüberblick.....................................................96

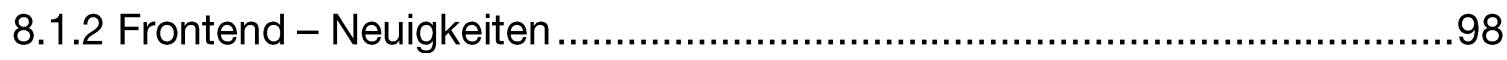

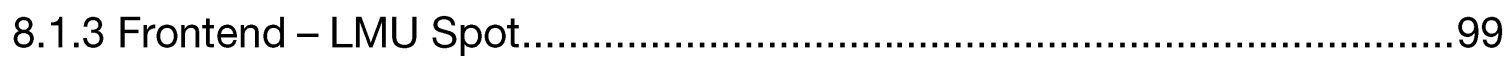

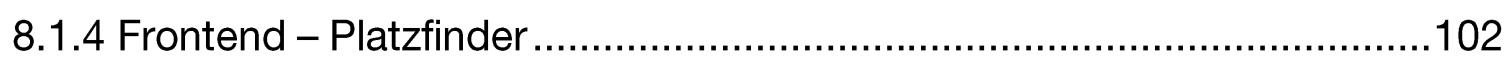

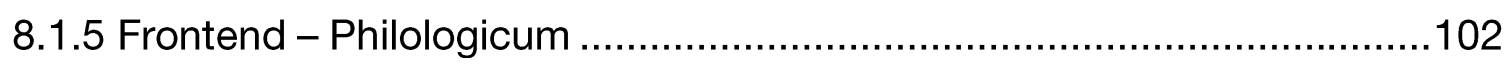

8.1.6 Frontend - Einstellungen................................................................103

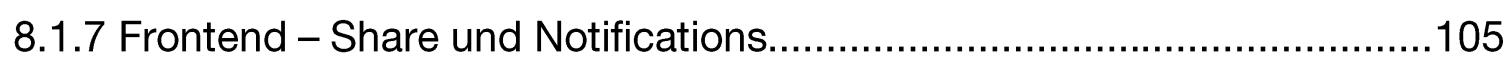

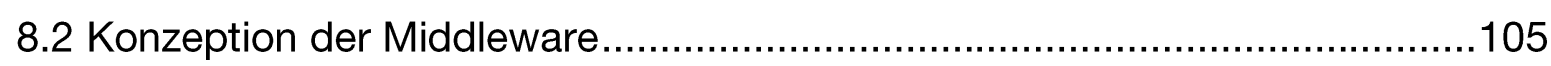

9. Prototypische Umsetzung „LMU Spot“.............................................................109

9.1 Prototypische Umsetzung der Middleware - „LMU Linker“...........................109

9.1.1 GUI der Webanwendung mittels Bootstrap .......................................110

9.1.2 Client- und serverseitige Logik mittels jQuery und PHP .......................111

9.1.3 Persistente Datensicherung mittels InnoDB.........................................113

9.1.4 Bereitstellung der Daten mittels einer API.............................................114

9.2 Prototypische Umsetzung des Frontends - „Philologicum“ ..........................115

9.2.1 Prototypische Umsetzung der Standortbestimmung.............................116

9.2.2 Prototypische Umsetzung der GUI .................................................119

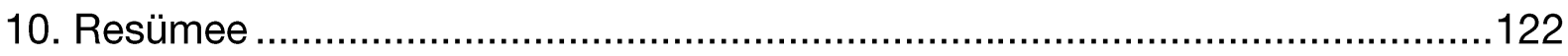

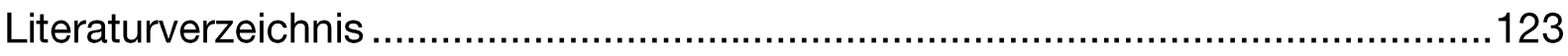

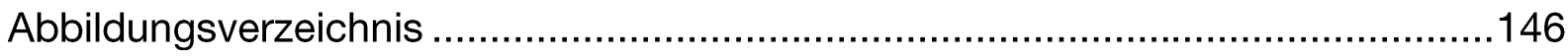

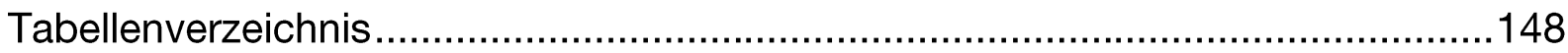

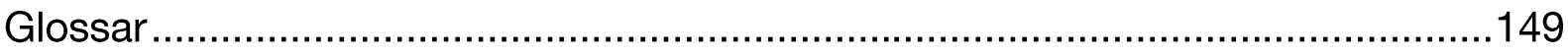

Eigenständigkeitserklärung ......................................................................157 


\section{Einleitung}

Unser Bedürfnis nach Informationen ist im Laufe der letzten Jahre stetig gestiegen. Begünstigt durch den Fakt, dass mobile Endgeräte, wie beispielsweise Smartphones, in unserer Gesellschaft nahezu omnipräsent sind, liegt es nahe, dass der Informationssuchende seinen persönlichen Assistenten zur Bewältigung der Informationsflut als Filter einsetzt. ${ }^{1}$ Eine Möglichkeit unser Informationsbedürfnis zu selektieren, ist der Bezug zu unserem aktuellem Standort. An dieser Stelle kommen Systeme zur Positionsbestimmung ins Spiel. Analysten der Unternehmensberatung ORBIS AG bescheinigen allein dem Markt für Indoor Positioning Systeme (IPS) eine verheißungsvolle Zukunft:

„Global Indoor Positioning and Indoor Navigation (IPIN) Market to Grow at a CAGR Of 58.90\% During The Period 2017-2021،

Mit diesen überdurchschnittlichen Wachstumsraten ist die Themensparte Locationbased Services (LBS) in den weltweiten wirtschaftlichen Märkten stark umkämpft. Auch in Deutschland nutzen ca. 77 Prozent der Unternehmen standortbasierte Informationen. ${ }^{4}$ Aufgrund der gesellschaftlichen Akzeptanz und des monetären Erfolges findet das Themenspektrum insbesondere in der aktuellen Forschung einen breiten Anklang. Der goldene Weg zur Beantwortung der Frage, wie der Standort beispielsweise in Innenräumen am treffendsten bestimmt werden kann, ist auch im Jahr 2018 noch längst nicht abschließend beantwortet. Bei näherer Betrachtung der Thematik scheinen sich diverse Rahmenbedingungen, Anforderungen, Lösungsmöglichkeiten und letztendlich die Umsetzung als zu diffizil, zu komplex oder auch zu variabel einem geplanten Vorhaben in den Weg zu stellen. Doch zunächst steht grundsätzlich die Frage im Mittelpunkt, warum sollten nicht auch Bibliotheken von dem aktuellen Trend und den neuartig erforschten Technologien der Standortbestimmung profitieren?

Die Frage nach einer möglichen Partizipation in dem Themenfeld „standortbasierte Informationen" hat die Abteilung der Informationstechnologie der Universitätsbibliothek (UB) der Ludwig-Maximilians-Universität München (LMU) für sich bereits positiv beantwortet. Anlass ist der Neubau des Philologicums, ein modernes,

\footnotetext{
${ }^{1}$ Vgl. Goldmedia GmbH Strategy 2014, S. 39.

${ }^{2}$ CAGR ist eine Abkürzung des englischen Begriffs "Compound Annual Growth Rate" und bedeutet übersetzt so viel wie "durchschnittliche jährliche Wachstumsrate".

${ }^{3}$ Thomson Reuters 2017.

${ }^{4}$ Vgl. Feigl 2018.
} 
dienstleistungsorientiertes und barrierefreies Fachzentrum für die Fakultät der Sprach- und Literaturwissenschaft, im Herzen von München. Als Ergänzung zu dem bisher geplanten Dienstleistungsportfolio soll ein System konzipiert werden, dass die Nutzer des zukünftigen Fachzentrums in den Mittelpunkt stellt und mit standortbasierten Informationen versorgen soll.

Ziel der Arbeit ist es, ein solches System zu entwerfen und dessen Machbarkeit anhand einer prototypischen Umsetzung zu demonstrieren. Um dieses geplante Vorhaben zu erreichen, ist die Separierung in mehrere Teilstationen, die sich in den einzelnen Kapiteln wiederfinden, notwendig. So ist zum Beispiel die Beantwortung der Frage nach der passendsten Technologie im Hinblick auf die Positionsbestimmung für die UB ein zentrales Element. Nach Möglichkeit soll der Leser alle notwendigen Informationen zum Verständnis des Themenkomplexes dieser Abhandlung entnehmen können.

Die Masterarbeit beginnt zunächst damit die allgemeinen Rahmenbedingungen zum Neubau des Philologicums zu beleuchten, damit ein Eindruck von dem Projekt vermittelt werden kann. In den Kapiteln 3 bis 5 beschäftigt sie sich mit den theoretischen Grundlagen, die das Spektrum rund um LBS und IPS beleuchten. Dieses Fundament ist essenziell, da mit dem Forschungsprojekt der aktuelle Stand der Technik möglichst umfänglich repräsentiert werden soll. Darüber hinaus bilden die gesammelten Erkenntnisse die Basis für zahlreiche Schlussfolgerungen und Ableitungen, ohne die einzelne getroffene Entscheidungen sonst nicht nachvollziehbar wären.

Die Anforderungs- und Zielgruppenanalyse in Kapitel 6 ist schließlich der Beginn der praktischen Ausführung und Umsetzung der zuvor geschaffenen Grundlage. Sie bestimmt die genauen Bedingungen, an denen sich das zukünftige System zu messen hat. Stütze der Analyse ist neben der Entwicklung von Personas mit konkreten User Stories, die Eruierung eines Anforderungskataloges, der auf die konkreten Bedürfnisse der Auftraggeberin abgestimmt ist. Das anschließende Kapitel aggregiert die gewonnen theoretischen Erkenntnisse und die aufgestellten Anforderungskriterien letztlich zu einer kaskadierenden Evaluation. Untermauert wird diese durch verschiedene praktische Testszenarien, mit dem Ziel die geeignetste Technologie für die indoor Positionsbestimmung festzulegen.

Die Kapitel 8 und 9 bilden schlussendlich die Synthese des zuvor erarbeiteten Kenntnisstandes und konzipieren zum einen ein System, das mit standortbasierten 
Informationen arbeitet und setzt dieses zum anderen prototypisch um. Schwerpunkt ist klar das Frontend des Systems. Damit dieses bereits in der Phase des Prototyps funktionell ist, wird jedoch auch die Middleware in die Entwicklungsphase mit einbezogen. In die Beschreibung des Prototyps fließen neben dem aktuellen Entwicklungsstand gleichermaßen Impulse für zukünftige Möglichkeiten zur Erweiterung und Verbesserung des Systems ein. 


\section{Der Neubau des Philologicums der Universitätsbibliothek}

Im Wintersemester 2017/2018 sind an der LMU München insgesamt 50.918 Studenten immatrikuliert. ${ }^{5}$ Gemessen an der absoluten Zahl der Studierenden ist die Fakultät für Sprach- und Literaturwissenschaften die größte Fachrichtung der LMU. ${ }^{6}$ Für Studierende hält die Fakultät ein differenziertes Spektrum an Studienrichtungen bereit. Diese reichen „Von der Indogermanistik, Finnougristik und klassischen Philologie über Anglistik, Amerikanistik, Slavistik, Romanistik und Italianistik bis zur Skandinavistik und Komparatistik. "'7

Zur Gewährleistung einer optimalen Versorgung mit wissenschaftlichen Fachinformationen jeglicher Art ist eine funktional orientierte Bibliotheksstruktur in Form einer Fachbibliothek unerlässlich. Aufgrund der historischen Bedingungen ${ }^{8}$ und einem organisch gewachsenen Bestand, ist die gedruckte Literatur auf über 10 Standorte in insgesamt 6 verschiedenen Gebäuden verteilt. ${ }^{9}$ Um eine Bündelung der gesamten Literatur und Schaffung eines zentralen Arbeits- und Lernortes für die Studenten zu erreichen, startete Anfang 2013 die Planung zum Neubau des Philologicums. ${ }^{10}$

Bereits mit Klärung der Standortfrage ist ein Zeichen für die Bedeutung und Wichtigkeit des Neubaus gesetzt worden. Das Philologicum soll an prominenter Stelle im Herzen von München Bestandteil eines der bedeutendsten Straßenzüge der Stadt werden. Das zu renovierende Gebäude in der Ludwigstraße 25, das „1833-35 in der Ära Ludwigs I. von Friedrich von Gärtner erbaut wurde ${ }^{\text {st1 }}$, soll im Zuge der geplanten Restaurationen zu einem modernen und barrierefreien Dienstleistungszentrum umfunktioniert werden. Mitte 2014 ${ }^{12}$, gut ein Jahr nach offiziellen Beginn des Projektes konnte der Architektenwettbewerb erfolgreich abgeschlossen werden und mit dem Siegerentwurf bekam ein erfahrenes österreichisches Architektenbüro den Zuschlag, dass bereits zuvor einige Projekte für die öffentliche Hand erfolgreich realisiert hat. ${ }^{13}$

\footnotetext{
${ }^{5}$ Vgl. Ludwig-Maximilians-Universität München 2018.

${ }^{6}$ Vgl. Ludwig-Maximilians-Universität München.

${ }^{7}$ Ebd.

${ }^{8}$ Schüller-Zwierlein 2014, S. 314.

${ }^{9}$ Vgl. Ludwig-Maximilians-Universität München 2017c.

${ }^{10}$ Vgl. Ludwig-Maximilians-Universität München 2013.

${ }^{11}$ Schüller-Zwierlein 2014, S. 313.

${ }^{12} \mathrm{Vgl}$. ebd.

${ }^{13}$ Weitere Informationen unter: http://www.cn-architekten.at/
} 


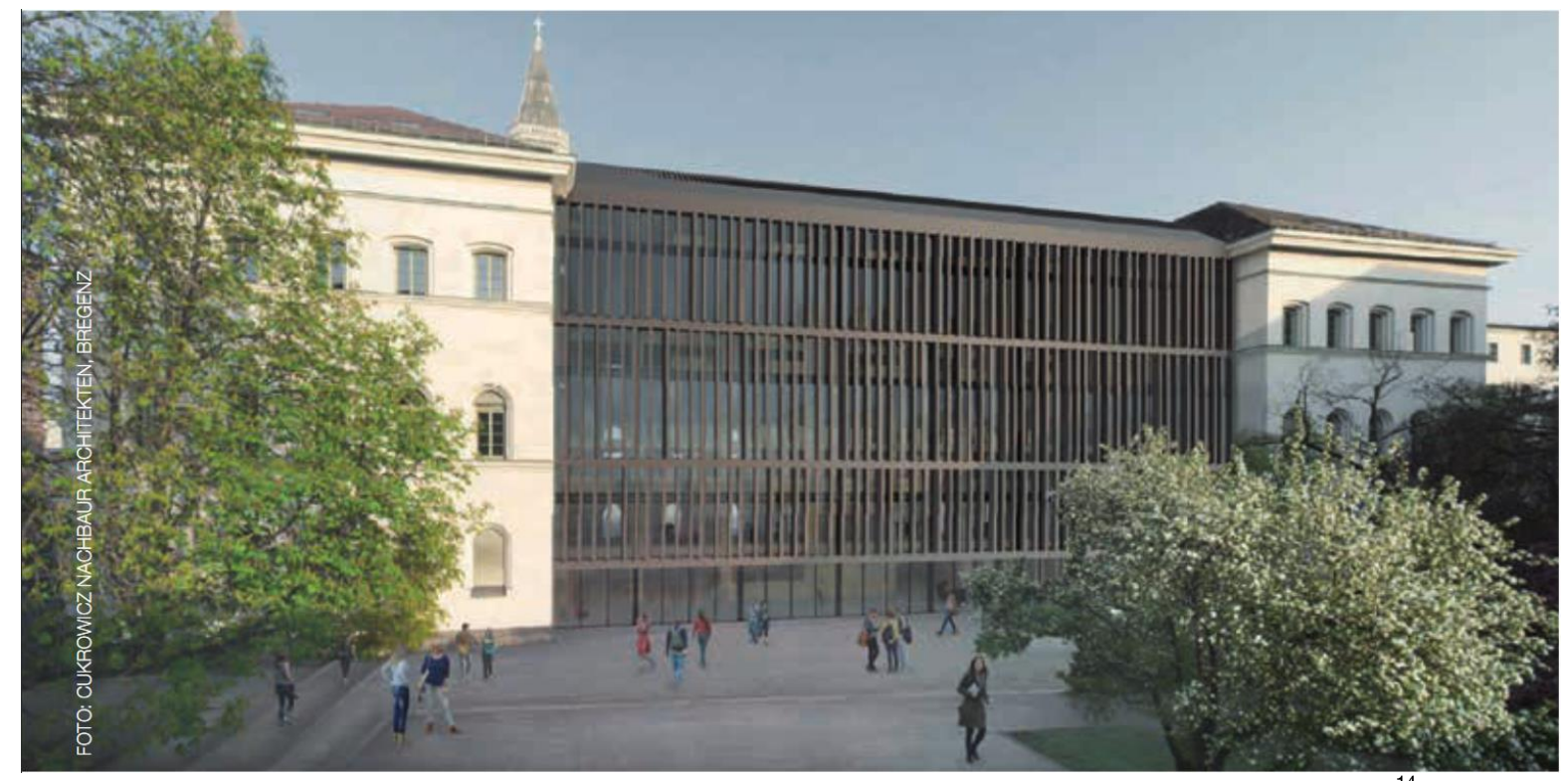

Abbildung 1: Entwurf der Fassade zum Innenhof - Fink Thurner und Cukrowicz Nachbaur ${ }^{14}$

Das zukünftige Gebäude sollte sich nach dem Entwurf des Architektenbüros Fink Thurnher und Cukrowicz Nachbaur in insgesamt vier Doppelstockwerke aufteilen.

Das Erdgeschoss und die drei Studienebenen bestehen jeweils aus einer zweigeschossigen Haupt- mit zentraler Galerieebene. Im Keller kommt noch ein Magazin mit Anlieferungszone hinzu. Durch den in sich geschlossenen Schnitt der einzelnen Geschosse soll ein guter Schallschutz garantiert werden. Die doppelstöckigen Etagen, wie in Abbildung 2 gezeigt ermöglichen durch ihre Bauweise zugleich eine luftige Raumatmosphäre.

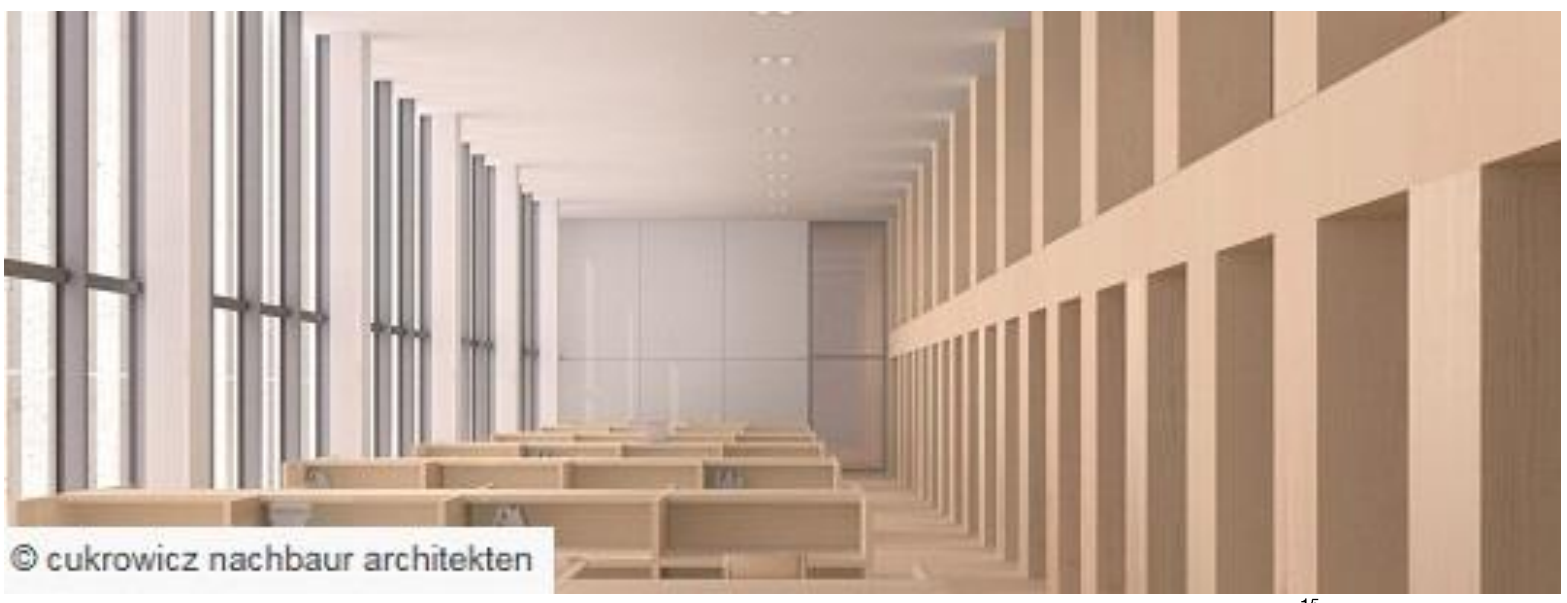

Abbildung 2: Entwurf der zweigeschossigen Hauptebene mit zentraler Galerieebene ${ }^{15}$

Der Entwurf des Architekturbüros sieht eine Gliederung der Nutzungsfläche in drei Zonen vor. ${ }^{16}$ Im Hinblick auf das Nutzungskonzept sollen zum einen Bereiche

\footnotetext{
${ }^{14}$ Quelle: Ludwig-Maximilians-Universität München 2017c.

${ }^{15}$ Quelle: Ludwig-Maximilians-Universität München 2017c.

${ }^{16}$ Vgl. Ludwig-Maximilians-Universität München 2017a.
} 
entstehen, in denen die Förderung der Konzentration, beispielsweise beim Lesen oder Lernen, im Fokus steht, wie auf Abbildung 3 skizziert. Zum anderen sollen Räume zum Arbeiten und Diskutieren in Gruppen entstehen. Abgerundet wird das Konzept durch eine verbindende Zone, die zum zwanglosen Aufenthalt und informellen Austausch, als ein Raum der Begegnung, aber auch als Rückzugsort fungieren soll.

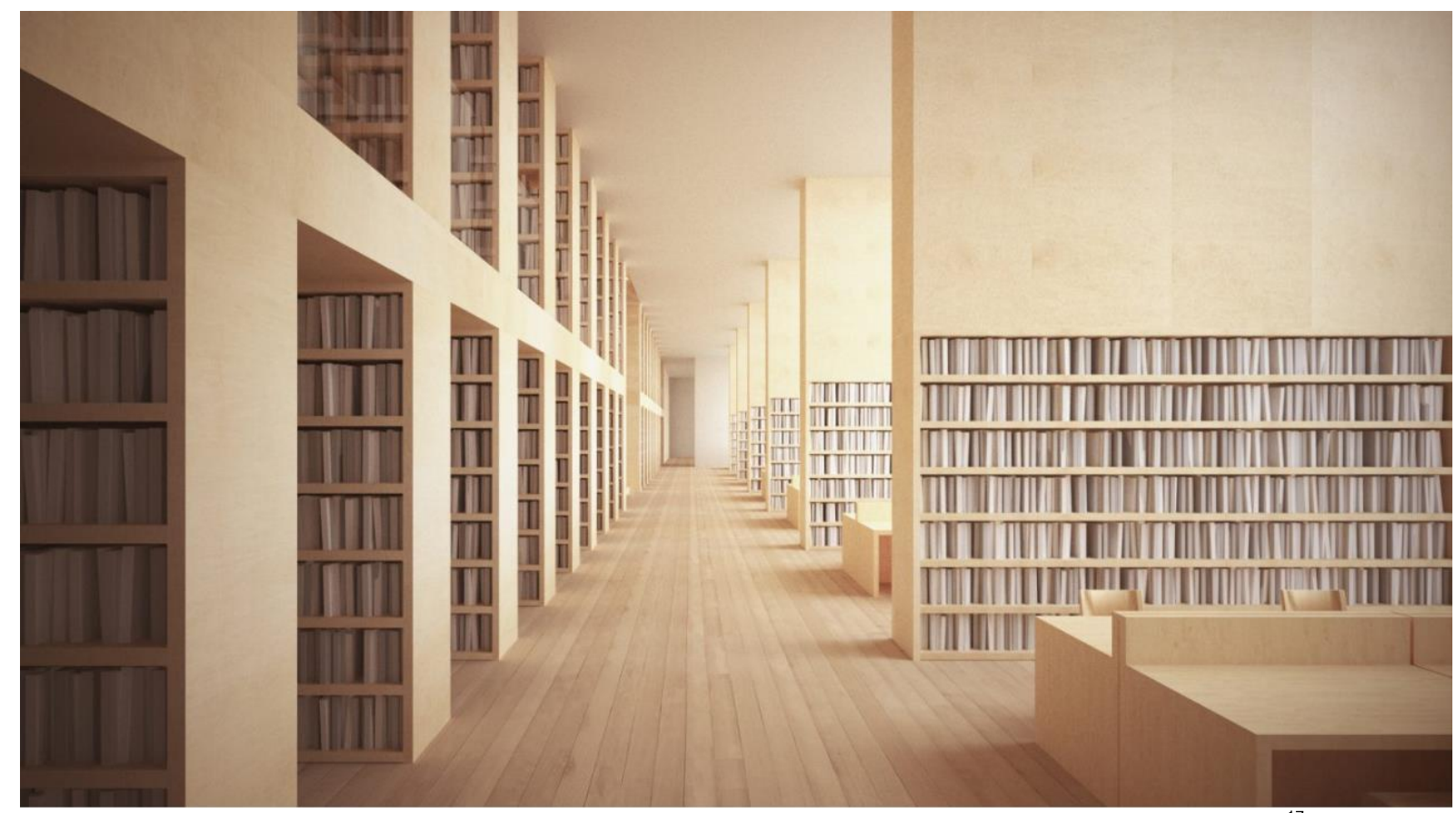

Abbildung 3: Entwurf der zweigeschossigen Hauptebene mit separierten Arbeitsbereichen ${ }^{17}$

Gerade im universitären Umfeld, aber auch in anderen Sparten des Bibliothekswesens, hat sich die Bibliothek als Institution einer „radikalen Transformation vom Buchorientierten Wissensspeicher hin zum Nutzer-orientierten ,Dritten-Ort"c18 unterzogen. Dieser funktionsorientierten Umwandlung trägt gleichfalls der Neubau des Philologicums Rechnung.

So wird neben dem Bestand von ca. 420.000 Bänden auf der Hauptnutzungsfläche von $6.000 \mathrm{~m}^{2}$ gleichermaßen Raum für insgesamt etwa 700 Arbeitsplätzen, in Form von Einzel- und Gruppenarbeitsplätzen, geschaffen. ${ }^{19}$ Damit wird den Studierenden ein Ort erbaut, der sowohl für konzentrierte Einzelarbeit, als auch für das Lernen und den Austausch in Gruppen geeignet sein soll. Für einen flexiblen Einsatz der vorwiegend mobilen Arbeitsgeräte der Studentinnen und Studenten soll im gesamten Gebäude die Verfügbarkeit von Internet via Wireless Local Area Network (WLAN)

\footnotetext{
${ }^{17}$ Quelle: PSA Publishers Ltd. 2014.

${ }^{18}$ Griebel et al. 2015, S. 95.

${ }^{19}$ Vgl. Ludwig-Maximilians-Universität München 2017c.
} 
sichergestellt sein. Nach der Planung auf dem Reißbrett wurden im Sommer 2015 die ersten sichtbaren Schritte zur Entstehung des Philologicums getan. Die bisher im historischen Gebäude der Ludwigstraße untergebrachten Institute für Romanische und italienische Philologie, samt der zugehörigen Teilbibliotheken sowie die Bibliothek und Studiobühne der Theaterwissenschaft sind in Ausweichgebäude bzw. an ihre neuen Standorte verlagert worden. ${ }^{20}$

Im Jahr 2016 erfolgte die Baufreigabe ${ }^{21}$ des Neubaus und der teilweise Abriss ${ }^{22}$ des bisherigen Gebäudes. Ein erster Meilenstein im Bauvorhaben ist die offizielle Grundsteinlegung am 24. November 2016 gewesen. ${ }^{23}$ Nach einem planmäßigen Verlauf des Rohbaus konnte am 25.07.2017 das Richtfest, unter anderem unter Anwesenheit des Staatsministers Dr. Ludwig Spaenle gefeiert werden. ${ }^{24}$ Im Anschluss wird nun zum aktuellen Stand der Innenausbau des Philologicums durchgeführt.

Durch die Bearbeitung und Fertigstellung einzelner Bauabschnitte ist der visuelle Fortschritt des Projektes für den Betrachter offensichtlich stets greifbar. Im Hintergrund sind darüber hinaus jedoch, scheinbar unsichtbar, zahlreiche Akteure mit dem unmittelbaren Erfolg des Vorhabens betraut. Das bibliothekarische Projektteam der UB begleitet beispielsweise neben zahlreichen Musterungen und Abnahmen des Baufortschrittes parallel die Planung des benötigten Mobiliars. ${ }^{25}$ Des Weiteren ist neben der Bearbeitung im physischen Raum zugleich eine digitale „[...] Vereinheitlichung und Bereinigung der Katalogdaten [...] der einziehenden elf Bibliotheksstandorte [.... ${ }^{\text {:26 }}$ von dem Projektteam eingeleitet worden. Das mit einem Budget von 33 Millionen Euro ${ }^{27}$ veranschlagte Projekt „Neubau Philologicum“, soll im Frühsommer 2019 bezogen werden. ${ }^{28}$

\footnotetext{
${ }^{20}$ Vgl. Ludwig-Maximilians-Universität München.

${ }^{21}$ Vgl. Unger 2016.

${ }^{22}$ Vgl. Tostmann 2016.

${ }^{23}$ Vgl. Ludwig-Maximilians-Universität München 2016.

${ }^{24} \mathrm{Vgl}$. Ludwig-Maximilians-Universität München 2017b.

${ }^{25}$ Vgl. Ludwig-Maximilians-Universität München Universitätsbibliothek 2018, S. 11.

${ }^{26}$ Ebd., S. 12.

${ }^{27}$ Vgl. Unger 2016.

${ }^{28}$ Vgl. Ludwig-Maximilians-Universität München Universitätsbibliothek 2018, S. 12.
} 


\section{Location-based Services}

\subsection{Definition}

LBS oder übersetzt ortsbezogene Dienste werden in der Literatur der vergangenen Jahre zum Teil recht diffizil und stark differenziert in ihrer Grundform abgegrenzt. ${ }^{29}$ In verschiedensten Definitionen häuft sich ein Bezug zu dem Begriff "Context-Awareness“, der allgemein als Oberbegriff für LBS verwendet werden kann. Dey und Abowd definieren den Begriff "Context" hierbei wie folgt:

"Context is any information that can be used to characterize the situation of an entity. An entity is a person, place, or object that is considered relevant to the interaction between an user and an application, including the user and applications themselves. ${ }^{\text {‘30 }}$

Darauf aufbauend formulieren beide Autoren eine allgemeine Beschreibung für den Begriff „Context-Aware“:

„A system is context-aware if it uses context to provide relevant information and/or services to the user, where relevancy depends on the user's task. ${ }^{\text {iB1 }}$

Spiekermann aggregiert diese allgemeinen Definitionen und stellt LBS als eine Teilmenge von "Context-Awareness", ,as services that integrate a mobile device's location or position with other information so as to provide added value to a user ${ }^{432}$ heraus. Wissenschaftliche Studien belegen, dass neben der Generierung eines informationellen Mehrwehrtes ${ }^{33}$ ferner die Ortsunabhängigkeit ${ }^{34}$ der angebotenen Information essentiell für den Erfolg des Dienstes sind. Neben diesen beiden Faktoren ist darüber hinaus die Verquickung mit dem mobilen Endgerät des Nutzers ${ }^{35}$ ebenfalls ein entscheidendes Kriterium für dessen tatsächliche Verwendung im Alltag.

\footnotetext{
${ }^{29}$ Vgl. Bauer et al. 2009, S. 208.

${ }^{30}$ Abowd et al. 1999, S. $304 f$.

${ }^{31}$ Ebd., S. 307.

${ }^{32}$ Spiekermann op. 2004 , S. 10 .

${ }^{33}$ Vgl. Bauer et al. 2009, S. 207.

${ }^{34} \mathrm{Vgl}$. Unni und Harmon 2007, S. 28.

${ }^{35}$ Vgl. Werner 2014, S. 4 und Steiniger et al. 2006, S. 3.
} 
Vor allem im Hinblick auf die Eigenschaften Lokalisierbarkeit, Erreichbarkeit und Ortsunabhängigkeit, die gerade im mobilen Umfeld relevant sind, zeigt die Klassifikation nach Tschersich in Abbildung 4, dass sich Smartphones und Mobiltelefone durch eine entsprechend hohe Auszeichnung dieser Merkmale hervorheben. ${ }^{36}$

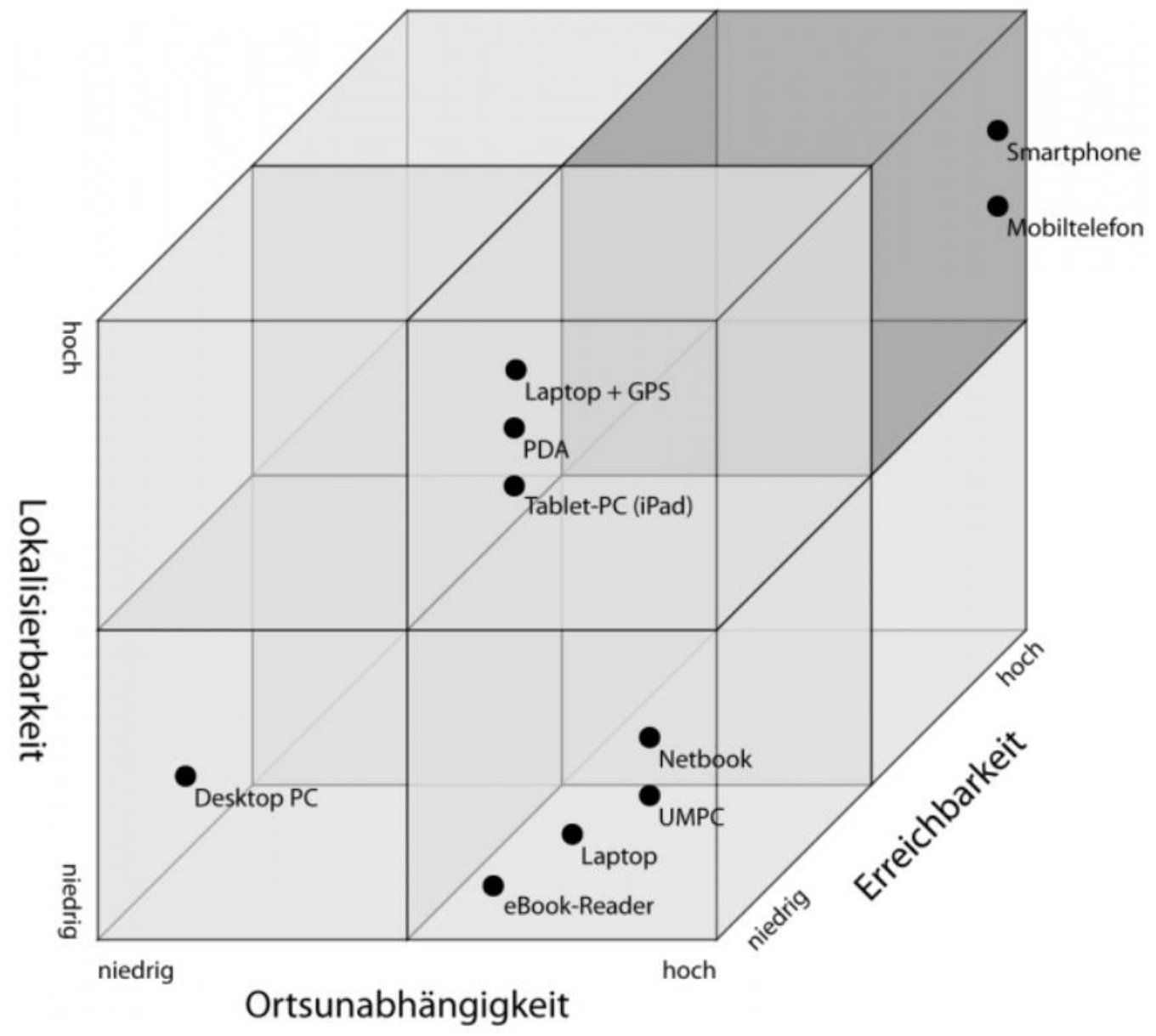

Abbildung 4: Einstufung mobiler Endgeräte (Tschersich) ${ }^{37}$

Gerade das Smartphone hat sich im Laufe der Jahre immer mehr zum persönlichen Assistenten und ständigen Begleiter entwickelt ${ }^{38}$, dementsprechend ist die Nutzung dieser Geräteklasse über verschiedene Altersstufen hinweg intensiver geworden. ${ }^{39}$ Diese Entwicklung begünstigt generell die Bekanntheit, Akzeptanz und Nutzung von divergenten LBS $^{40}$ über verschiedene Nutzergruppen hinweg. ${ }^{41}$

\footnotetext{
${ }^{36} \mathrm{Vgl}$. Tschersich 2010.

${ }^{37}$ Quelle: Tschersich 2010.

${ }^{38}$ Vgl. Bitkom e.V. 2017, S. 53.

${ }^{39} \mathrm{Vgl}$. Tesche 2018, S. 6.

${ }^{40} \mathrm{Vgl}$. Huang und Gartner 2018, S. 1.

${ }^{41}$ Vgl. Lopez 2013, S. 6.
} 


\subsection{Architektur von Location-based Services}

Ausgehend von der vorangegangenen Definition entwirft Spiekermann ein allgemeines LBS Kommunikationsmodell mit drei verschiedenen Schichten. Diese bilden gemeinsam ein Geoinformationssystem, dass einen LBS anbieten kann. ${ }^{42}$

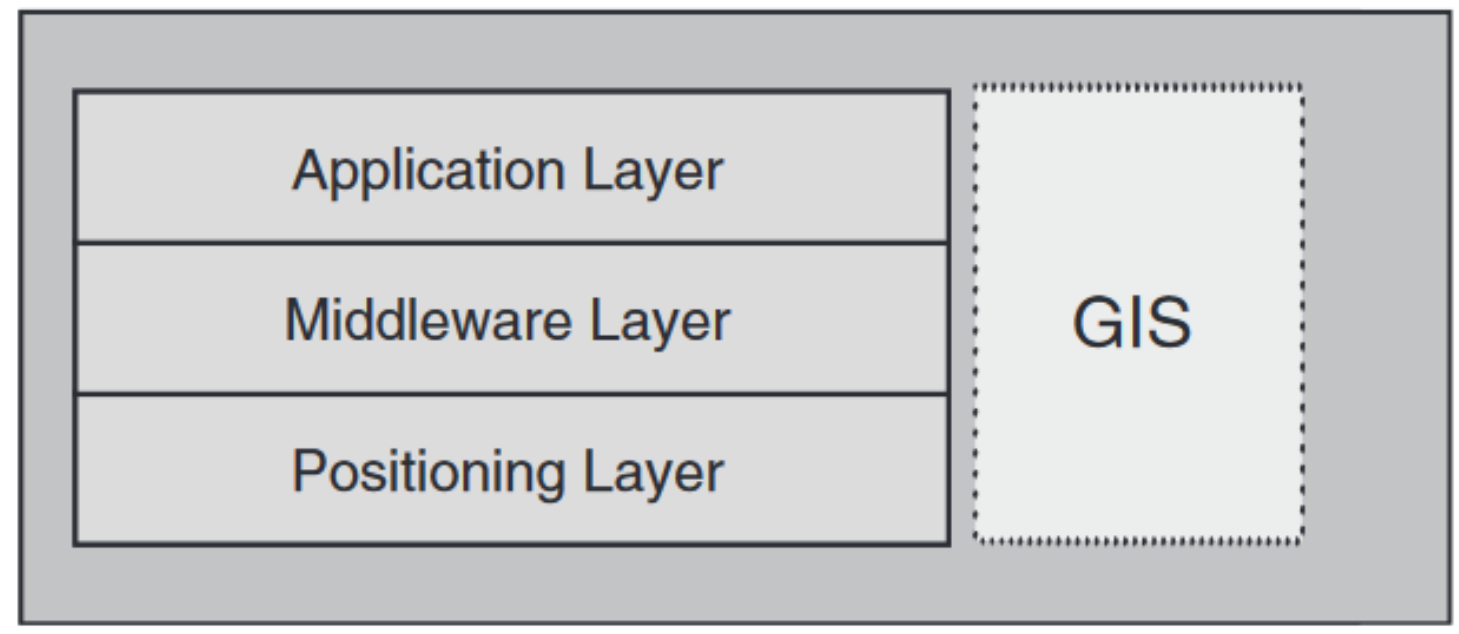

Abbildung 5: LBS Kommunikationsmodell nach Spiekermann ${ }^{43}$

Jede der drei Schichten wird auf eine spezifische Aufgabe zur Bereitstellung des LBS abstrahiert. In welcher Form die einzelnen Schichten in einer späteren Umsetzung implementiert werden lässt dieses Modell offen.

Der Positioning Layer übernimmt im Kontext des Kommunikationsmodells, die Aufgabe den Standort des Nutzers zu bestimmen. Im Wesentlichen soll dies mit der Hilfe von „position determination equipment ${ }^{* 44}$, also Technologien zur Standortbestimmung erfolgen. Weiterführende Informationen zum aktuellen Stand der Technik können dem Kapitel 5 dieser Arbeit entnommen werden. Das Ergebnis der Positionsbestimmung wird im folgenden Verarbeitungsschritt über eine Schnittstelle entweder direkt an den Application Layer oder zunächst über einen Middleware Layer geschickt. Der Middleware Layer kann als eine optional aufbereitende Prozessschicht in die Systemarchitektur eingebaut werden. Häufig sind LBS-Systemarchitekturen derartig komplex, dass es sinnvoll ist durch die Integration einer Zwischenschicht die Komplexität zu reduzieren. Beispielsweise können Prozesse zur Positionsbestimmung

\footnotetext{
${ }^{42}$ Vgl. Spiekermann op. 2004, S. 23.

${ }^{43}$ Quelle: Ebd.

${ }^{44}$ Ebd., S. 22.
} 
sehr tief in der eigenen oder der Systemarchitektur eines Drittanbieters verankert sein, sodass die Prozessierung einzelner Datenpakete nicht ohne weiteres möglich ist. ${ }^{45}$ Die Applikationsschicht, oder auch Application Layer genannt, verarbeitet, wie in anderen Kommunikations- und Referenzmodellen auch, die prozessierten Daten. Eine weitere Funktion ist die Integration der Daten in den jeweiligen Anwendungsdienst. Das heißt diese Schicht stellt entsprechende Datenstrukturen und Protokolle bereit, damit die Daten abschließend in der Anwendung verarbeitet werden können. ${ }^{46}$

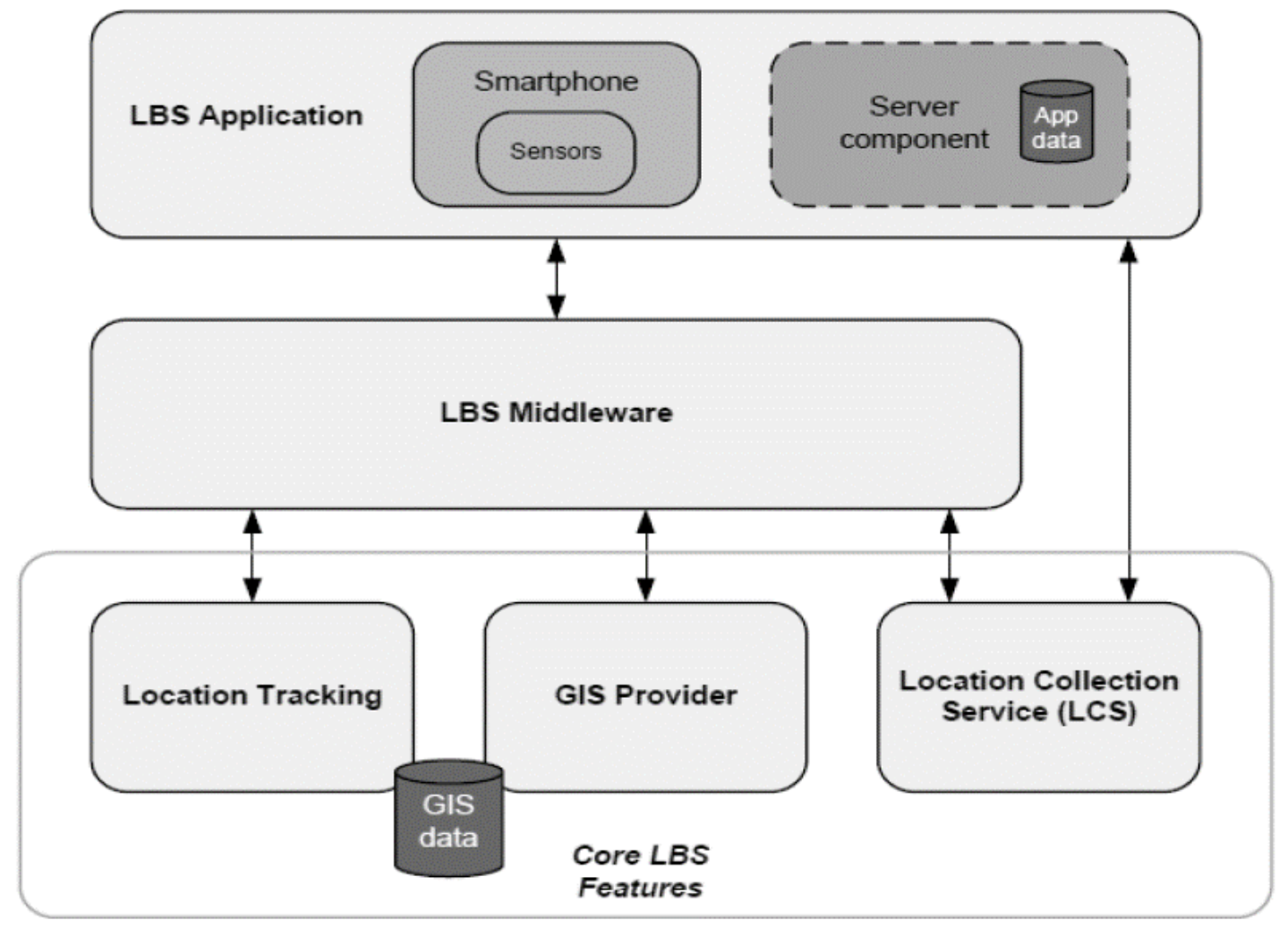

Abbildung 6: granulare LBS-Komponenten ${ }^{47}$

Wie Abbildung 6 beispielhaft anführt kann das generalisierte Modell nach Spiekermann um eine Granularität im Hinblick auf einzelne Komponenten des LBS-Systems ergänzt werden. ${ }^{48}$ An dieser Stelle zeichnet sich die Anwendungsschicht beispielsweise durch eine innere Aufteilung in eine Client-Server-Architektur aus. Zum einen verarbeitet ein Smartphone als Client mit diversen Sensoren, die über die Middleware oder über den „Core LBS Features“-Layer bzw. Positioning Layer zur Verfügung

\footnotetext{
${ }^{45}$ Vgl. Spiekermann op. 2004, S. 23.

${ }^{46}$ Vgl. Meinel und Sack 2012, S. 61.

${ }^{47}$ Quelle: Kushwaha und Kushwaha 2011, S. 16.

${ }^{48}$ Ebd.
} 
gestellten Daten. Zum anderen ist in den Application Layer parallel eine Server Komponente integriert, die unter anderem spezifische Anwendungsdaten vorhalten kann.

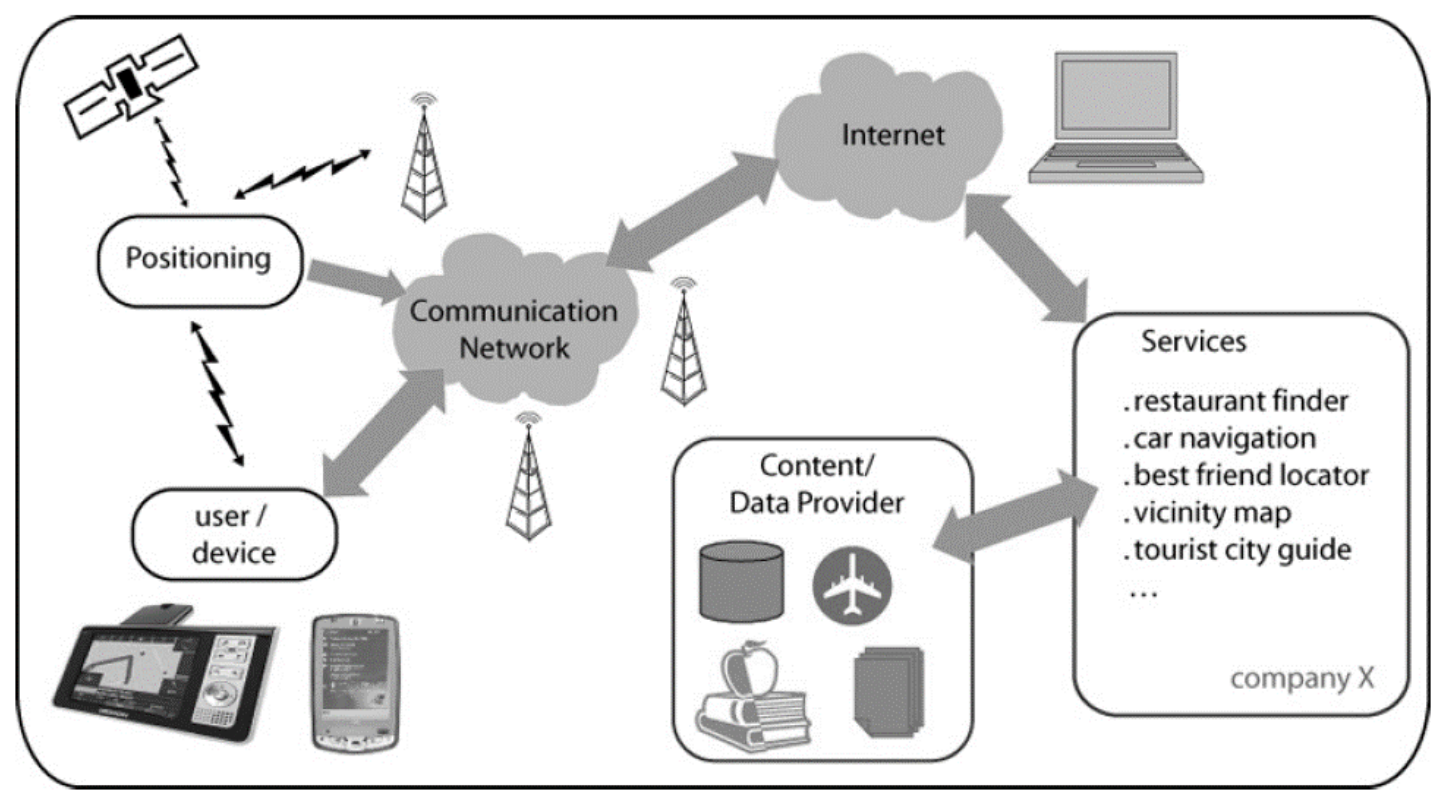

Abbildung 7: Zusammenspiel und Informationsfluss einzelner LBS-Komponenten ${ }^{49}$

In Anlehnung an Steiniger lässt sich auch aus heutiger Sicht eine detaillierte LBSArchitektur im Hinblick auf den Informationsfluss wie in Abbildung 7 dargestellt beschreiben ${ }^{50}$ und ist im Prinzip eine zusätzliche Erweiterung, des von Spiekermann entworfenen Kommunikationsmodells. Es zeigt jedoch, dass eine gewisse Grundinfrastruktur vorhanden sein muss, um einen LBS abbilden zu können.

So wird als eine Komponente der Nutzer mit seinem mobilen Endgerät benötigt, der über ein Positionierungsverfahren seinen Standort bestimmt. Dieser Standort wird anschließend über ein Kommunikationsnetzwerk an den Anbieter des LBS übermittelt. Dieser wiederum muss nicht zwingend standortbezogene Informationen in einer eigenen Datenhaltung gesammelt und gespeichert haben, sondern kann diesen Prozess zu einem Content / Data Provider auslagern. Im Zuge einer Anfrage des Nutzers können über entsprechende Schnittstellen die relevanten Daten abgefragt werden. Liegen die aggregierten Informationen zu dem übermittelten Standort vor, liefert der LBSProvider abschließend seine Daten über das Kommunikationsnetzwerk an das Endgerät des Nutzers zurück. Je nach Anwendungsszenario bzw. gegebener Systemarchitektur können einzelne Komponenten und Teilabschnitte des Workflows

\footnotetext{
${ }^{49}$ Quelle: Steiniger et al. 2006, S. 16.

${ }^{50}$ Ebd.
} 
zusammengelegt oder in anderer Reihenfolge wiedergegeben werden. Wie bereits angedeutet kann der Content / Data Provider zum Beispiel gleichzeitig der LBS-Provider sein. Zahlreiche Szenarien können jedoch auf diese aufgezeigte Grundstruktur abstrahiert werden.

LBS können im Hinblick auf die Klassifikation unterschiedlicher Dienstarten in zwei verschiedene Kategorien unterteilt werden. ${ }^{51}$ Zum einen können die Dienste reaktiv im Zuge eines Pull-Verfahrens dem Nutzer zur Verfügung gestellt werden und zum anderen im Rahmen eines proaktiven Push-Dienstes. Darüber hinaus kann es bei der Bereitstellung der Informationen für den Nutzer Mischformen beider Verfahren geben.

Bei einem reaktiven Pull-Dienst wird die Interaktion vom Benutzer initiiert und die Information auf direkte Anfrage vom LBS ausgeliefert. Bei diesem Verfahren handelt es sich um eine synchrone Kommunikation, in der der Nutzer eine Anfrage an den Dienst stellt, die dann vom System verarbeitet und mit einer entsprechend ortsabhängigen Information beantwortet wird. Beispielsweise kann hier das Anfragen von Informationen an einem Point of Interest (POI) aufgeführt werden, bei dem der Nutzer aktiv eine Anfrage am jeweiligen Standort stellt.

Im Gegenteil dazu stellt der Benutzer bei einem proaktiven Push-Dienst keine explizite Anfrage an das System, viel mehr wird im Vorfeld eine ortsabhängige Information für eine bestimmte Positionierung hinterlegt und bei Betreten eines speziellen Bereiches über den Dienst ausgeliefert. Folglich handelt es sich bei dem proaktiven Push-Dienst um eine asynchrone Kommunikation zwischen Nutzer und LBS. Als Beispiel kann hier angeführt werden, dass der Nutzer ein Gebäude betritt, das im Vorhinein als Eventbereich im System definiert worden ist. Dieses Betreten löst nun die Auslieferung einer entsprechenden Information aus, ohne dass eine direkte Initiierung durch den Nutzer notwendig ist. Allein das Betreten des vorher definierten Bereiches reicht aus, damit das System proaktiv standortbezogene Informationen bereitstellt. ${ }^{52}$

\footnotetext{
${ }^{51}$ Vgl. Steiniger et al. 2006, S. 5.

${ }^{52}$ Vgl. DATACOM Buchverlag GmbH 2017.
} 


\subsection{Ausgewählte Location-based Services in Bibliotheken}

In diesem Kapitel soll untersucht werden welche LBS Bibliotheken derzeit anbieten. Die untersuchten Beispiele erheben keinen Anspruch auf Vollständigkeit, sondern sollen vielmehr einen selektiven Querschnitt von potentiellen Anwendungsszenarien abbilden. Vor allem von Interesse ist im Rahmen der Arbeit welche Erkenntnisse aus den jeweiligen Projekten, beispielsweise in Bezug auf verwendete Technologien, gewonnen worden sind.

Im Zuge des Aufkommens von LBS wie Foursquare ${ }^{53}$, Facebook Places ${ }^{54}$ oder Google Maps $^{55}$, haben einige Bibliotheken in Deutschland das Potential dieser Dienste für sich erkannt und entsprechend genutzt. ${ }^{56}$ Die Funktionalität des Angebotes richtete sich entsprechend an der von dem Anbieter zur Verfügung gestellten Dienstleistungspalette aus. Damit beschränkten sich zunächst die Möglichkeiten auf eine Form des digitalen Marketings für die Institution Bibliothek. ${ }^{57}$ Die Möglichkeiten der Individualisierung sind durch die Nutzung der Dienstleistungsinfrastruktur der Anbieter infolgedessen limitiert gewesen und dienten mehr dem experimentellen Erkunden der Möglichkeiten von LBS.

Im Laufe der letzten Jahre sind einige Bibliotheken den Schritt gegangen und haben sich ein eigenes Portfolio von LBS, zur Ergänzung ihres Dienstleistungsspektrums aufgebaut. Mit der Investition in den Ausbau von eigenem Know-How und Infrastruktur ist es vereinzelten Bibliotheken gelungen, abseits großer Anbieter von LBS individuelle Strukturen und Angebote zu schaffen. Wie die folgenden Projekte zeigen werden, sind sowohl komplette Eigenentwicklungen, als auch Kooperationen mit kleinen und mittleren Technologieunternehmen zu finden.

Erfahrungsgemäß sind meist Wissenschaftliche Bibliotheken, gerade im Experimentieren mit technologischen Innovationen Öffentlichen Bibliotheken einen Schritt voraus. Begünstigt durch den Vorteil meist am aktuellen Puls von Wissenschaft und Technik, durch die zu versorgende Universität bzw. Hochschule zu sein, kann ein aktiver Wissenstransfer zwischen den Institutionen gelebt werden.

\footnotetext{
${ }^{53}$ Weitere Informationen zu dem Dienst unter: https://de.foursquare.com/

${ }^{54}$ Weitere Informationen zu dem Dienst unter: https://de-de.facebook.com/places/

${ }^{55}$ Weitere Informationen zu dem Dienst unter: https://www.google.de/maps

${ }^{56}$ Vgl. Böhner 2012.

${ }^{57}$ Vgl. Vatter 2011.
} 
Vor allem Öffentliche Bibliotheken verwenden LBS im Hinblick auf die Umsetzung des Gamification-Prinzips. Gamification meint nicht das Spielen an sich, sondern viel mehr die „...Übernahme der Mechaniken, Modelle und Denk- und Arbeitsweisen in NonGame-Kontexte. ${ }^{558}$ Stellvertretend sei an dieser Stelle Harri Ketamos Literaturrallye auf Basis der Near Field Communication (NFC)-Technologie genannt. ${ }^{59}$ Im Rahmen des Pilotprojektes zum Thema „Transmedia“ ist das Ziel die Untersuchung im Hinblick auf die Verknüpfung des analogen und digitalen Raumes gewesen. ${ }^{60}$

Die verwendete Technologie, in diesem Falle NFC, stand nicht im Fokus, sondern diente lediglich als verknüpfendes Bindeglied zum Transfer zwischen beiden Räumen. In dieser Rolle muss die verwendete Technologie vor allem primär ihre zugeordnete Funktion erfüllen. ${ }^{61}$ Ein Fazit, dass sich während der Umsetzung des Konzeptes herauskristallisiert hat ist folgendes gewesen: „[s]ome players also felt that scanning the books RFID tag with the tablets NFC reader was bit difficult ${ }^{\text {“62 }}$.

Ein wesentlich breiteres Spektrum an Projekten, in Bezug auf LBS ist im Bereich Wissenschaftlicher Bibliotheken zu finden. Die als sehr experimentierfreudig geltende Bibliothek des Karlsruher Instituts für Technologie hat mit der App „OnSitelnfo“63 einen ortsbezogenen Informationskanal für die KIT-Bibliothek geschaffen. ${ }^{64}$ Ursprünglich unter dem Namen „BibTip-Beacon“65 gestartet, sollte das Projekt, neben dem Wissensgewinn im Bereich IPS auf Basis der Bluetooth Low Energy (BLE)-Technologie, die bisherigen globalen Informationskanäle um eine digitale, lokale Komponente erweitern. ${ }^{66}$ Das Projekt wurde in Kooperation mit der vom KIT ausgegründeten BibTip $\mathrm{GmbH}^{67}$ realisiert. ${ }^{68}$

Die mobile Anwendung sollte hierbei im Vorbeigehen zuvor im Gebäude platzierte Beacons ohne eine temporäre Verzögerung erkennen. ${ }^{69}$ Der Nutzer sollte dann in der App eine Benachrichtigung über „eine ortsbezogene, noch aktuelle Information“"70

\footnotetext{
${ }^{58}$ Deeg Christoph 2017, S. 315.

${ }^{59}$ Vgl. Ketamo et al. 2014, S. 3.

${ }^{60}$ Vgl. ebd., S. 8.

${ }^{61}$ Vgl. ebd., S. 6.

${ }^{62}$ Ebd., S. 7.

${ }^{63}$ Karlsruher Institut für Technologie 2017.

${ }^{64} \mathrm{Vgl}$. Dierolf 2018, S. 256.

${ }^{65}$ Dierolf 2017, S. 371.

${ }^{66} \mathrm{Vgl}$. ebd.

${ }^{67}$ Vgl. Karlsruher Institut für Technologie 2009.

${ }^{68} \mathrm{Vgl}$. Dierolf 2018, S. 260.

${ }^{69}$ Vgl. Dierolf 2018, S. 256.

${ }^{70}$ Ebd.
} 
erhalten. Neben einem Frontend ist darüber hinaus ein funktional umfangreiches Backend zur Verwaltung der bereitgestellten Informationen konzipiert und umgesetzt worden. ${ }^{71}$ Im Rahmen der Umsetzung und damit verbunden dem Zusammenspiel der einzelnen Technologien sind Verlauf des Projektes jedoch zahlreiche Hindernisse aufgekommen, die zu Beginn nicht absehbar gewesen sind.

Als Hauptursache für das Scheitern des Projektes wird insbesondere das nicht fehlerfreie Zusammenspiel einzelner technischer Komponenten angeführt. ${ }^{72}$ Vor allem die zeitlich sehr variable Erkennungsrate einzelner Beacons vom Operating System (OS), sowohl bei Ausführung der App im Vorder-, als auch Hintergrund, hat letztendlich zum Scheitern des Unterfanges geführt. ${ }^{73}$ BLE-Beacons können sehr schnell vom mobilen OS erkannt werden, müssen es aber nicht. In Android 9 baut Google mit „Adaptive Battery" eine Funktion in das OS ein, die wenig genutzte Apps im Hintergrund schneller schlafen legt und baut damit den Doze-Stromsparmodus weiter aus. ${ }^{74}$ Dadurch sollte die Erkennungsrate gerade im Hintergrund laufender und nur moderat genutzter Anwendungen weiter sinken. Darüber hinaus ist neben dem Wartungsaufwand der Beacons, beispielsweise in Form von regelmäßigen Intervallen zur Kontrolle des Akkustandes, auch die Tatsache als Nachteil empfunden worden, dass der Nutzer zusätzlich die Bluetooth (BT)-Schnittstelle an seinem Smartphone aktivieren muss. ${ }^{75}$ Zahlreiche Internetportale empfehlen zur Schonung des Akkus die Schnittstelle, in Form des leichtgewichtigen Verbrauchers BT, zur Erhöhung der Standby-Laufzeit des Smartphones auszuschalten. ${ }^{76}$ Sollte der Anwender keine entsprechenden Peripherie Geräte auf Basis von BT, wie beispielsweise Kopfhörer oder »Wearables« nutzen, liegt es nahe, dass BT folglich deaktiviert wird.

Ein weiteres interessantes Pilot-Projekt, in Bezug auf die Vermittlung standortbasierter Online-Informationen, hat die UB Bern im Frühsommer 2016 unter dem Namen „CUBe“ konzipiert und angewendet. Im Zuge der Sanierung einer Teilbibliothek der UB ist der Versuch unternommen worden, den analogen und digitalen Raum

\footnotetext{
${ }^{71}$ Vgl. Dierolf 2018, S. 258.

${ }^{72} \mathrm{Vgl}$. ebd.

${ }^{73}$ Vgl. ebd., S. 257.

${ }^{74}$ Vgl. Porteck 2018, S. 29.

${ }^{75}$ Vgl. Dierolf 2018, S. 258.

${ }^{76}$ Eine Recherche nach den Stichwörtern „Smartphone Akku sparen“ oder „Smartphone Laufzeit verlängern" bringt zahlreiche Tipps- und Hilfeseiten einschlägiger Technikportale zum Vorschein, bei denen sich in der Regel die Empfehlung zum Deaktivieren nicht permanent genutzter Schnittstellen, wie beispielsweise BT findet.
} 
miteinander zu verknüpfen und damit dem Bibliothekspublikum elektronische Ressourcen besser zugänglich zu machen. ${ }^{77}$ Aufgrund extrinsischer Faktoren, wie Knappheit personeller und finanzieller Ressourcen, stand eine einfache technische und vor allem kostengünstige Umsetzung des Konzeptes im Vordergrund. ${ }^{78}$ Die Basis der Konzeptionierung bilden, wie im vorangegangenen Beispiel, gleichfalls BT-Beacons. ${ }^{79}$

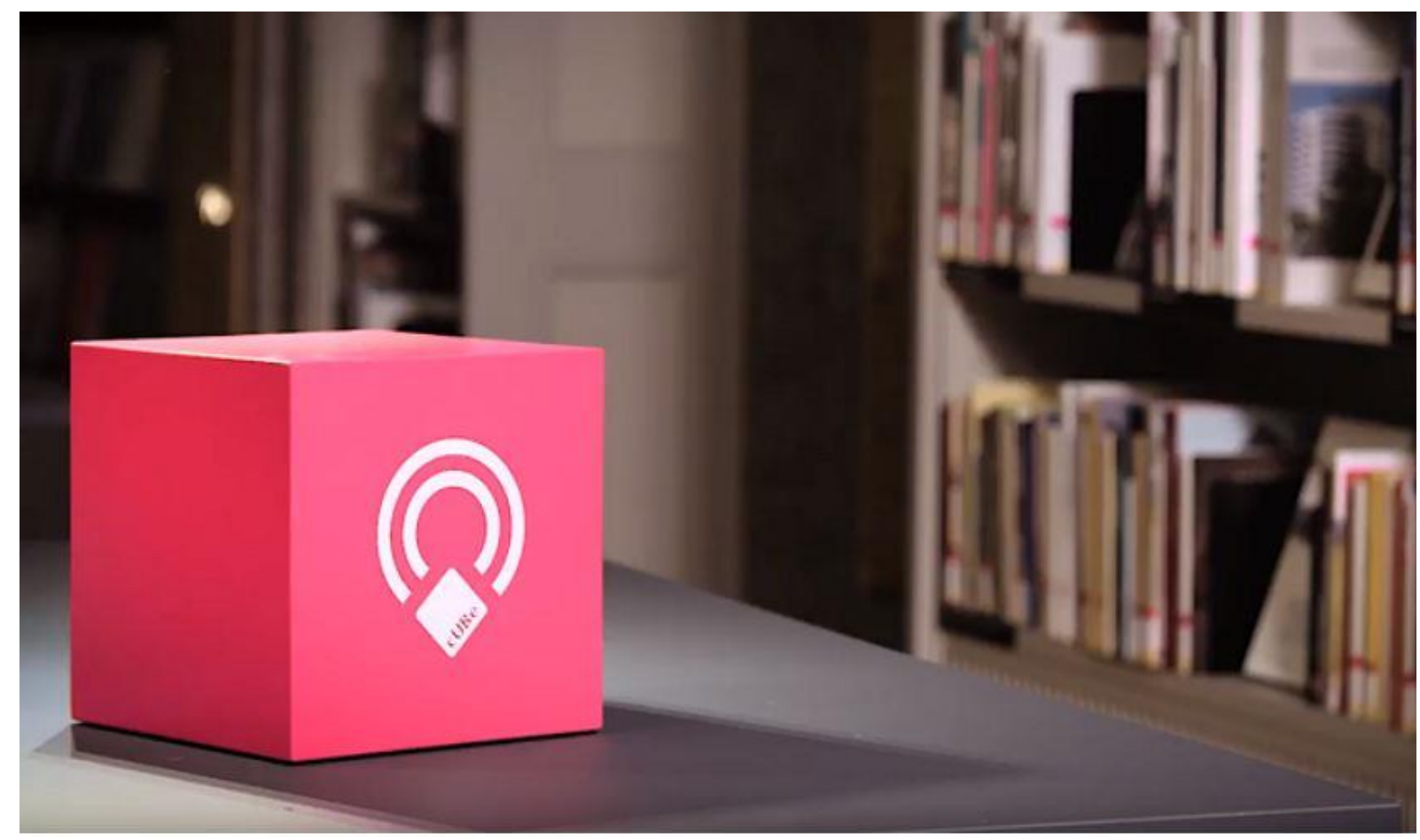

Abbildung 8: Promowürfel des Projektes $\mathrm{cUBe}^{80}$

Konträr ist jedoch, dass der Nutzer mittels der in Abbildung 8 gezeigten „Promowürfe/“81 direkt signalisiert bekommt an dieser Stelle einen LBS, in Form eines Pull-Dienstes, in Anspruch nehmen zu können. Dieser integrale Bestandteil der Verknüpfung des physischen Raumes mit digitalen Inhalten ist gerade bei Pull-Diensten für die erfolgreiche Anwendung essentiell.

Auf die Entwicklung eines eigenen Frontends ist vollständig verzichtet worden, stattdessen hat man sich auf die Nutzung von 3rd-Party-Apps zur Erkennung der Beacons festgelegt. ${ }^{82}$ Ein umfangreiches Backend zur Administration der lokal angebotenen Informationen ist ebenfalls nicht entwickelt worden. Alternativ hat man einen eigenen

\footnotetext{
${ }^{77}$ Vgl. List und Kirgus 2017, S. 63.

${ }^{78}$ Vgl. ebd., S. 66.

${ }^{79}$ Vgl. ebd., S. 67.

${ }^{80}$ Quelle: Universität Bern 2018.

${ }^{81}$ Landolt und Reto 2016, S. 16.

${ }^{82}$ Vgl. List und Kirgus 2017, S. 67.
} 
URL-Shortener verwendet, um die Beacons mit den jeweils informationstragenden bestehenden, bzw. neu entwickelten Websites zu verknüpfen. ${ }^{83}$

Die im Beispiel des KIT-Projektes genannten Nachteile haben auch im Hinblick auf cUBe weiterhin ihre Gültigkeit. Um den LBS für potentielle Nutzer noch einfacher zugänglich zu machen, laufen momentan Bestrebungen im Hinblick auf cUBe 2.0 alle Informationsressourcen gleichermaßen via QR-Code anzubieten. ${ }^{84}$ Abschließend lässt sich sagen, dass die UB Bern im Gegensatz zur KIT-Bibliothek die BT Technologie für eine andere Dienstart des LBS nutzt und damit verbunden versucht einigen Nachteilen aus dem Weg zu gehen. Jedoch soll schlussendlich die Einbindung einer weiteren Technologie eben diese systemimmanenten Beeinträchtigungen weitestgehend kompensieren.

Bezüglich der QR-Code-Technologie haben sich bereits einige deutsche UBs dazu entschieden, ähnlich wie im Beispiel der UB Bern aufgezeigt, den analogen Raum, um eine digitale Komponente zu erweitern. Dies bietet sich insbesondere für beispielsweise Lehrbuchsammlungen, Zeitschriftenfreihandmagazine oder Semesterapparate an. Die digitale Komponente besteht zumeist aus der Weiterleitung auf eine entsprechend elektronische Variante der analogen Form. An dieser Stelle ist oft kein umfangreiches IPS für diesen LBS konzipiert, sondern lediglich eine experimentelle Integration in die bestehende Infrastruktur vorgenommen worden. Als Frontend wird zumeist eine externe mobile Anwendung, beispielsweise einer der zahlreichen QR-CodeScanner verwendet. Als Beispiele für diesen Kontext lassen sich aus dem Bereich der Wissenschaftliche Bibliotheken die KIT-Bibliothek ${ }^{85}$, die UB der Freien Universität Ber$\operatorname{lin}^{86}$, die UB der Technischen Universität Hamburg ${ }^{87}$ oder die UB der Universität Augsburg $^{88}$ finden.

Darüber hinaus setzen daneben andere Bibliotheken im deutschen, aber auch internationalen Raum auf BT als Technologieträger für die Umsetzung individueller LBS. Die Bayerische Staatsbibliothek hat zum Beispiel mit der App „BSB-Navigator ${ }^{\star 89}$ auf

\footnotetext{
${ }^{83}$ Vgl. List und Kirgus 2017, S. 68.

${ }^{84} \mathrm{Vgl}$. List 2018.

${ }^{85}$ Vgl. Karlsruher Institut für Technologie 2013.

${ }^{86}$ Vgl. Berndt 2012.

${ }^{87}$ Vgl. Stabenau 2010.

${ }^{88}$ Vgl. Universitätsbibliothek Augsburg 2016.

${ }^{89}$ Weitere Informationen unter: https://play.google.com/store/apps/details?id=com.bsb.android.navigator
} 
Basis von BLE-Beacons ein IPS entwickelt, dass neben der Unterstützung von POI, vornehmlich der Navigation im historischen Gebäude der Bibliothek dient..$^{90} \mathrm{Im}$ amerikanischen Raum hat die UB der University of Illionois einen Navigations- und Empfehlungsdienst als LBS mit Hilfe von BLE-Beacons umgesetzt. ${ }^{91}$ Nach der erfolgreichen Detektion des Anwenderstandortes bekommt dieser aufgrund seiner Positionierung im Raum Vorschläge für weiterführende Titel aus dem elektronischen Portfolio der Bibliothek. ${ }^{92}$

Zusammenfassend lässt sich sagen, dass es im bibliothekarischen Bereich in den letzten Jahren vermehrt Projekte gegeben hat, die sich mit dem Angebot eines LBS in unterschiedlichen Dienstarten beschäftigt haben. Die Initiatoren haben vor allem, neben dem Mehrwert für Nutzerinnen und Nutzer, den Aufbau von eigenem KnowHow hinblickend auf IPS forciert. Derzeit omnipräsent im Bereich der Push-Dienstarten scheint die Nutzung von BT als Technologieträger zu sein. Alternative Technologien finden aktuell in der Bibliothekslandschaft nur geringen Anklang. Pull-Dienste werden zum Teil ebenso mit BLE-Beacons realisiert, hier finden sich jedoch gleichfalls vermehrt Beispiele im Bereich der wissenschaftlichen Bibliotheken, die in der Vergangenheit auf QR-Codes gesetzt haben. Für Öffentliche Bibliotheken scheinen neben der Verwendung von QR-Codes zur Realisierung, genauso andere Technologien wie NFC in Frage zu kommen.

Essentiell für den Erfolg eines LBS ist darüber hinaus die Einhaltung der im Kapitel 3.1 genannten Eckpfeiler eines solchen Dienstes. Sollte die Anwendung beispielsweise keinen informationellen Mehrwert für den Nutzer haben, oder nur schwer zugänglichsein, dann ist das Projekt meist zum Scheitern verurteilt. Des Weiteren lässt sich bei der Wahl des Frontends in den ausgewählten Beispielen eine Homogenität in der Konzeption der Dienste feststellen. An dieser Stelle ist in allen untersuchten Beispielen das Smartphone als primäre Basis für eine Realisierung des LBS-Frontends ausgewählt worden. Gegensätzlich hierzu zeichnet sich die Wahl bezüglich der architektonischen Implementierung des konkreten Systems aus. Haben einige der betrachteten Bibliotheken konkreten Vorstellungen bezüglich einer Administration zukünftiger Dienste, so sind andere wiederum mehr am experimentellen Charakter interessiert.

\footnotetext{
${ }^{90}$ Vgl. Ceynowa 2016, S. 15.

${ }^{91}$ Vgl. Hahn 2017, S. 9.

${ }^{92}$ Ebd., S. $13 f$.
} 
Für einen nachhaltigen Erfolg eines LBS, ohne dass dieser nach einiger Zeit wieder aus dem Dienstleistungsportfolio einer Bibliothek verschwindet, ist jedoch eine konzeptionelle Grundlage und fortlaufende Evaluierung des bestehenden Service zwingend notwendig. 


\section{Das Smartphone als Frontend für Location-based Services}

\section{1 Überblick Smartphonemarkt}

Die Nachfrage nach Smartphones ist selbst nach mehr als einer Dekade seit ihrer Einführung ungebrochen. Wie Abbildung 8 zeigt wurden allein in Deutschland im Jahr 2017 über 24 Millionen Geräte verkauft. ${ }^{93}$ Trotz zu erkennender leicht rückläufiger Absatzzahlen in Abbildung 9, bleibt die Umsatzentwicklung doch weiterhin auf einem konstanten Level. Interessant ist hierbei die zu beobachtende Tendenz zum »Phablet«.

Absatzentwicklung

Smartphones (inklusive Phablets) in Deutschland 2015-2017 in Millionen Stück

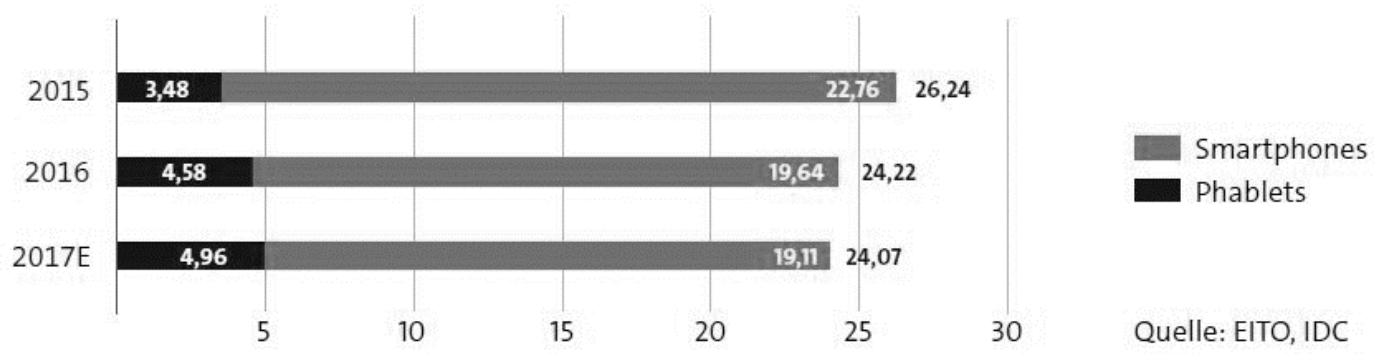

Abbildung 8: Absatzentwicklung Smartphones in Deutschland ${ }^{94}$

Diese Geräteklasse legte, selbst bei Stagnation des Smartphonemarktes, in den vergangenen Jahren fortwährend zu. Dies ist eine Tendenz, die bei der Entwicklung von mobilen Applikationen in den nächsten Jahren Berücksichtigung finden sollte.

\section{Umsatzentwicklung}

Smartphones (inklusive Phablets) in Deutschland 2015-2017 in Milliarden Euro

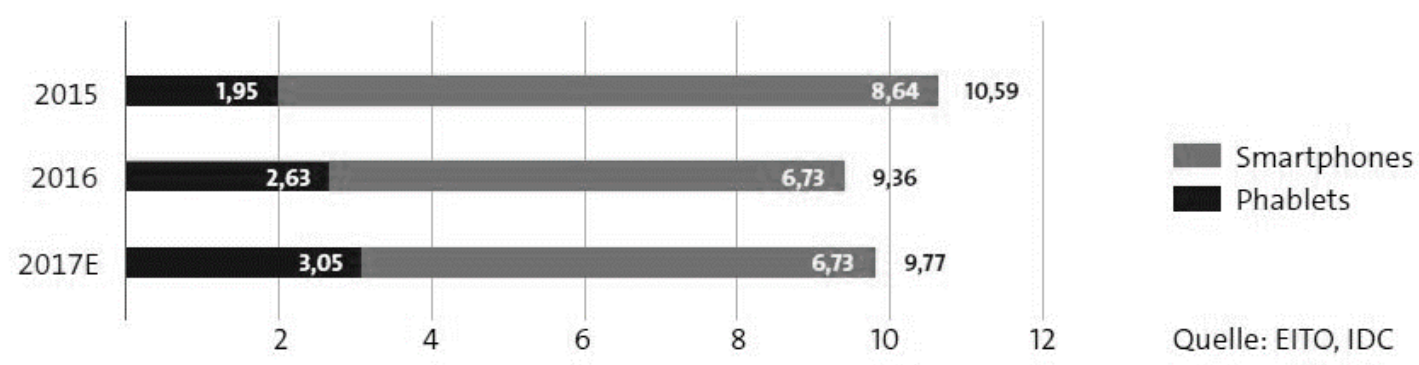

Abbildung 9: Absatzentwicklung Smartphones in Deutschland ${ }^{95}$

Das Marktvolumen für das Smartphone-Ökosystem wird in Deutschland für das Jahr 2018 auf etwa 33 Milliarden Euro prognostiziert. ${ }^{96}$ Neben der Tatsache, dass es sich

\footnotetext{
${ }^{93}$ Vgl. Bitkom e.V. 2017, S. 14.

${ }^{94}$ Quelle: ebd.

${ }^{95}$ Quelle: ebd.

${ }^{96}$ Vgl. Haas 2018, S. 2.
} 
um einen enormen wirtschaftlichen Markt handelt, ist ebenfalls entscheidend, dass allein in Deutschland acht von zehn Einwohnern ab 14 Jahren ein Smartphone nutzen. $^{97}$ In absoluten Zahlen ausgedrückt sind dies etwa 57 Millionen Personen.

In Kombination mit den gewonnenen Erkenntnissen aus Kapitel 3.1 und 3.3 bietet sich demnach das Smartphone als Basissystem im Hinblick auf die Konzeption und Implementierung eines LBS an. Vor allem bei der Entwicklung des Frontends liegt in dieser Plattform, aufgrund ihrer Marktkapitalisierung sowie Marktdurchdringung, Nutzungsquote und Zukunftsfähigkeit ein großes Potential für einen nachhaltigen Erfolg.

\subsection{Betriebssysteme von Smartphones}

Mit der Wahl des Smartphones als potentielle Plattform für die Entwicklung eines LBS kommt noch eine weitere Entscheidung auf den Entwickler zu. Die Auswahl des zukünftigen OS.

Wie die tabellarische Abbildung 10 zeigt, spielen neben Android und iOS andere OS kaum eine signifikante Rolle im Markt der Smartphones. Folglich beschränkt sich damit die Wahl auf die Optionen Android oder iOS, da diese am weitesten verbreitet sind. Obwohl Smartphones auf Basis von Android beinahe die Übermacht, gemessen an den absoluten Verkaufszahlen, haben, kann eine Entwicklung für iOS dennoch Sinn machen, da dadurch eine potentielle Zielgruppe nicht übergangen wird.

\begin{tabular}{|c|c|c|c|c|}
\hline Operating System & $\begin{array}{c}2017 \\
\text { Units }\end{array}$ & $\begin{array}{r}2017 \text { Market } \\
\text { Share }(\%)\end{array}$ & $\begin{array}{l}2016 \\
\text { Units }\end{array}$ & $\begin{array}{r}2016 \text { Market } \\
\text { Share }(\%)\end{array}$ \\
\hline Android & $1,320,118.1$ & 85.9 & $1,268,562.7$ & 84.8 \\
\hline iOs & $214,924.4$ & 14.0 & $216,064.0$ & 14.4 \\
\hline Other OS & $1,493.0$ & 0.1 & $11,332.2$ & 0.8 \\
\hline Total & $1,536,535.5$ & 100.0 & $1,495,959.0$ & 100.0 \\
\hline
\end{tabular}

Abbildung 10: Smartphone-Verkäufe nach $\mathrm{OS}^{98}$

\footnotetext{
${ }^{97}$ Vgl. Haas 2018, S. 3.

${ }^{98}$ Quelle: Gartner Says Worldwide Sales of Smartphones Recorded First Ever Decline During the Fourth Quarter of 20172018.
} 
Jedoch ist im Hinblick auf beide OS zu beachten, dass diese einer Fragmentierung bezüglich der installierten Versionen durch die Anwender unterliegen.

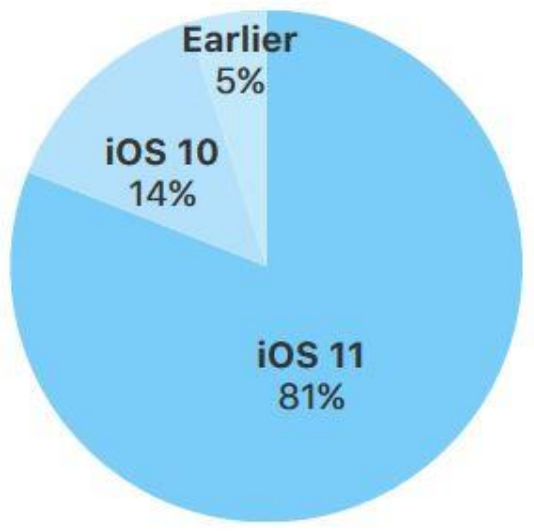

Abbildung 11: Fragmentierung iOS - Stand 31.05.2018

Es ist wichtig diese Fragmentierung zu kennen und in die Entwicklung mit einzubeziehen, da einige Features und Funktionen der beiden Plattformen erst mit einer bestimmten Versionierung Einzug halten. Android beispielsweise gibt bei jeder neuen Versionierung seines OS ein entsprechendes Application Programming Interface (API)-Level für die Entwickler frei. ${ }^{100}$ Die API-Level werden bei der Entwicklung benötigt, um auf das zugrunde liegende System und dessen Komponenten, wie Hardwareressourcen zu zugreifen. ${ }^{101}$

Die Fragmentierung beider OS unterscheidet sich stark voneinander. Das Diagramm in Abbildung 11 zeigt, dass diese bei iOS nicht so ausgeprägt ist im Vergleich zu Android. ${ }^{102}$ Dies liegt vor allem in der Art der Distribution. Das System iOS kann nur auf iPhones der Firma Apple verwendet werden, somit liegt es immer beim Nutzer, ob dieser ein Update für sein Smartphone installiert oder nicht. Einzige Beschränkung in diesem Hinblick sind die minimalen Installationsvoraussetzungen, die ein Update mit sich bringt. Sollte ein Gerät zu alt sein, dann wird es von Apple ab einem bestimmten Zeitpunkt nicht mehr mit Updates versorgt. Für die aktuelle Version 11 von iOS muss der Nutzer beispielsweise mindestens im Besitz eines iPhone 5 s sein. ${ }^{103}$

\footnotetext{
${ }^{99}$ Quelle: Vgl. Apple Inc. 2018a.

${ }^{100}$ Vgl. Schmidt 2018.

${ }^{101}$ Vgl. Google LLC 2018a.

${ }^{102}$ Vgl. Apple Inc. 2018a.

${ }^{103}$ Vgl. Apple Inc. 2018c.
} 


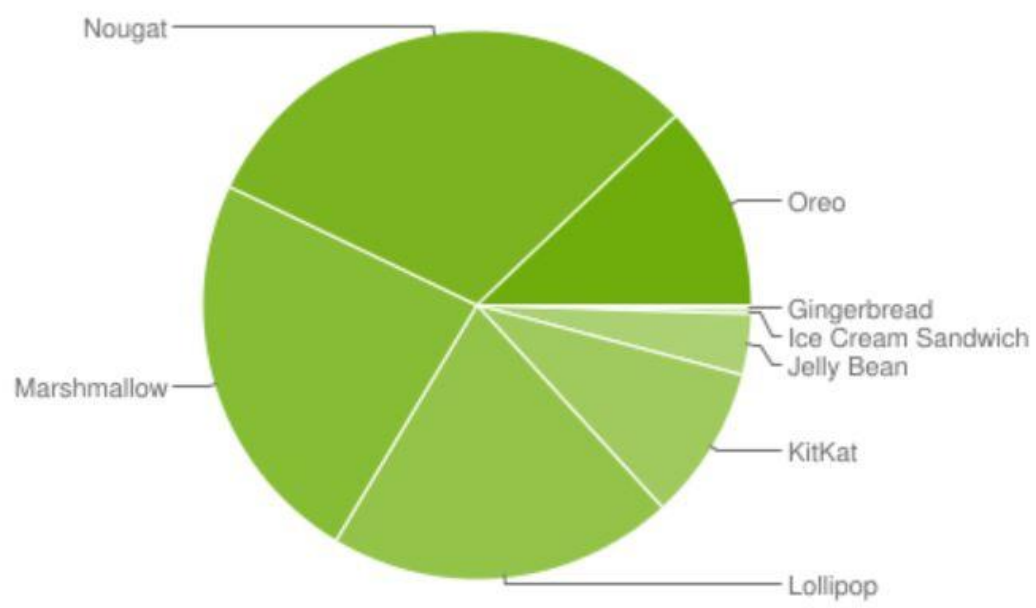

Abbildung 12: Fragmentierung Android - Stand 23.07.2018 ${ }^{104}$

Google als Betriebssystementwickler verfolgt in diesem Kontext eine andere Distributionsstrategie. Die jeweiligen Smartphone-Hersteller, wie Samsung, Huawei oder Sony können selbst entscheiden, ob, wann und wie sie kommende Updates von Android auf die Endgeräte der Käufer ausrollen. In der Regel benötigen Hersteller mehrere Monate bis ein von Google bereitgestelltes Update von Android auf die jeweiligen Geräte portiert wird. ${ }^{105}$

Darüber hinaus führt diese Art der Distribution zu einer stark ausgeprägten Fragmentierung von Android, wie in Abbildung 12 dargestellt. ${ }^{106}$ Diese zeigt, dass immerhin ein Großteil der Geräte noch nicht die aktuelle Version nutzen. Im Gegenteil die gegenwärtig neuste Version befindet sich mit einer relativen Durchdringungsquote von circa $10 \%$ deutlich in der Minderheit. Selbst die beiden zuvor erschienen Versionen von Android 6.0 und 7.0, respektive 7.1, teilen unter sich jeweils circa ein Viertel der in Betrieb befindlichen Smartphones auf.

Zumindest bezüglich Android könnte, dass im letzten Jahr gestartete Projekt „Treble“ endgültig die Problematik der Fragmentierung nachhaltig lösen. Abbildung 13 zeigt, dass durch die Einführung eines neuen Layers, dem „Vendor Interface“ ${ }^{\text {"107 }}$ in die Architektur von Android, die Portierung einer neuen Version für die Hersteller beschleunigt werden kann. ${ }^{108}$ Durch die Trennung von OS und Hardware, in Kombination mit der Kommunikation über den neuen Layer können Hersteller an ihren Treibern arbeiten,

\footnotetext{
${ }^{104}$ Quelle: Vgl. Google LLC 2018c.

${ }^{105}$ Beineke und Spier 2018, S. 120.

${ }^{106} \mathrm{Vgl}$. edb.

${ }^{107}$ Weitere Informationen unter: https://source.android.com/devices/architecture/

${ }^{108}$ Vgl. Minor 2017.
} 
während das OS schon vorher aktualisiert werden kann. ${ }^{109}$ Momentan müssen Hardwarehersteller bei jedem Versionsupdate von Android dieses vorher für ihre Geräte jeweils entsprechend portieren.

\section{Before Treble}

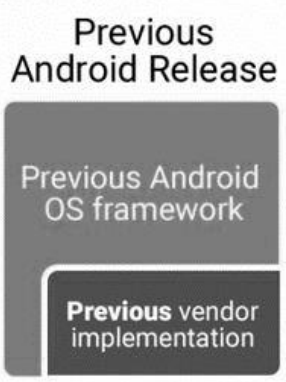

\section{With Treble}

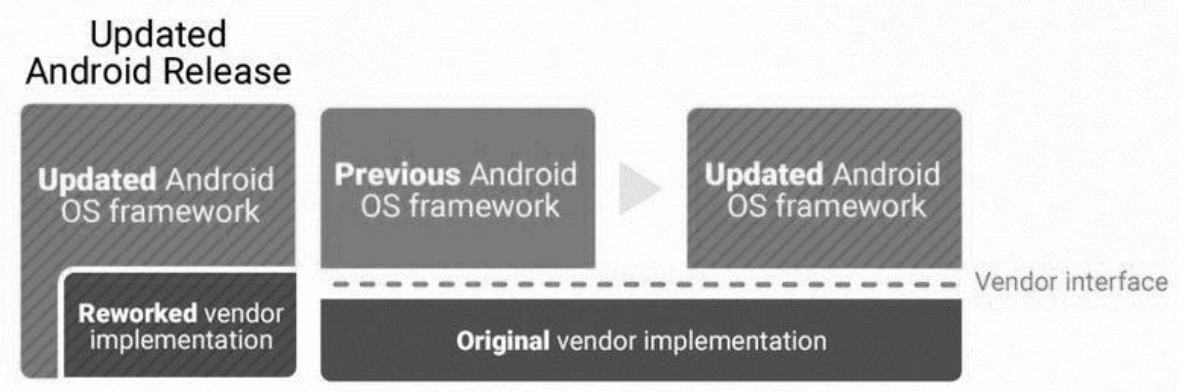

Abbildung 13: Ausschnitt der Architektur von Projekt „Treble ${ }^{\text {"110 }}$

Die Wahl des zukünftigen OS für eine mobile Anwendung ist ein wegweisender Meilenstein in einem Entwicklungsprojekt. Trotz der Bemühungen der Distributoren eine Fragmentierung innerhalb ihrer OS zu vermeiden, ist es wichtig eben diese im Rahmen einer Konzeption zu berücksichtigen.

\subsection{Sensorik in Smartphones}

Neben den bisher thematisierten Vorzügen eines Smartphones als Plattform für das Frontend eines LBS bietet es den Vorteil, mit einer breiten Palette an unterschiedlichen Sensoren ab Werk ausgestattet zu sein. Stellvertretend für die verschiedenen Produktpaletten, sei an dieser Stelle die Entwicklung der Sensorik in der Galaxy S Produktreihe des Smartphoneherstellers Samsung dargestellt. Die Grafik in Abbildung 14 verdeutlicht, dass die reine Anzahl der Sensoren in der Smartphonereihe kontinuierlich zugenommen hat. Damit verbunden ist zeitgleich eine erhöhte Funktionalität durch differenzierte Messwerte, auf die zurückgegriffen werden kann. Gerade für die Indoor- aber auch Outdoor-Positionsbestimmung kann diese Entwicklung nur begünstigend wirken.

\footnotetext{
${ }^{109}$ Vgl. Grüner 2017.

${ }^{110}$ Quelle: Vgl. Malchev 2017.
} 


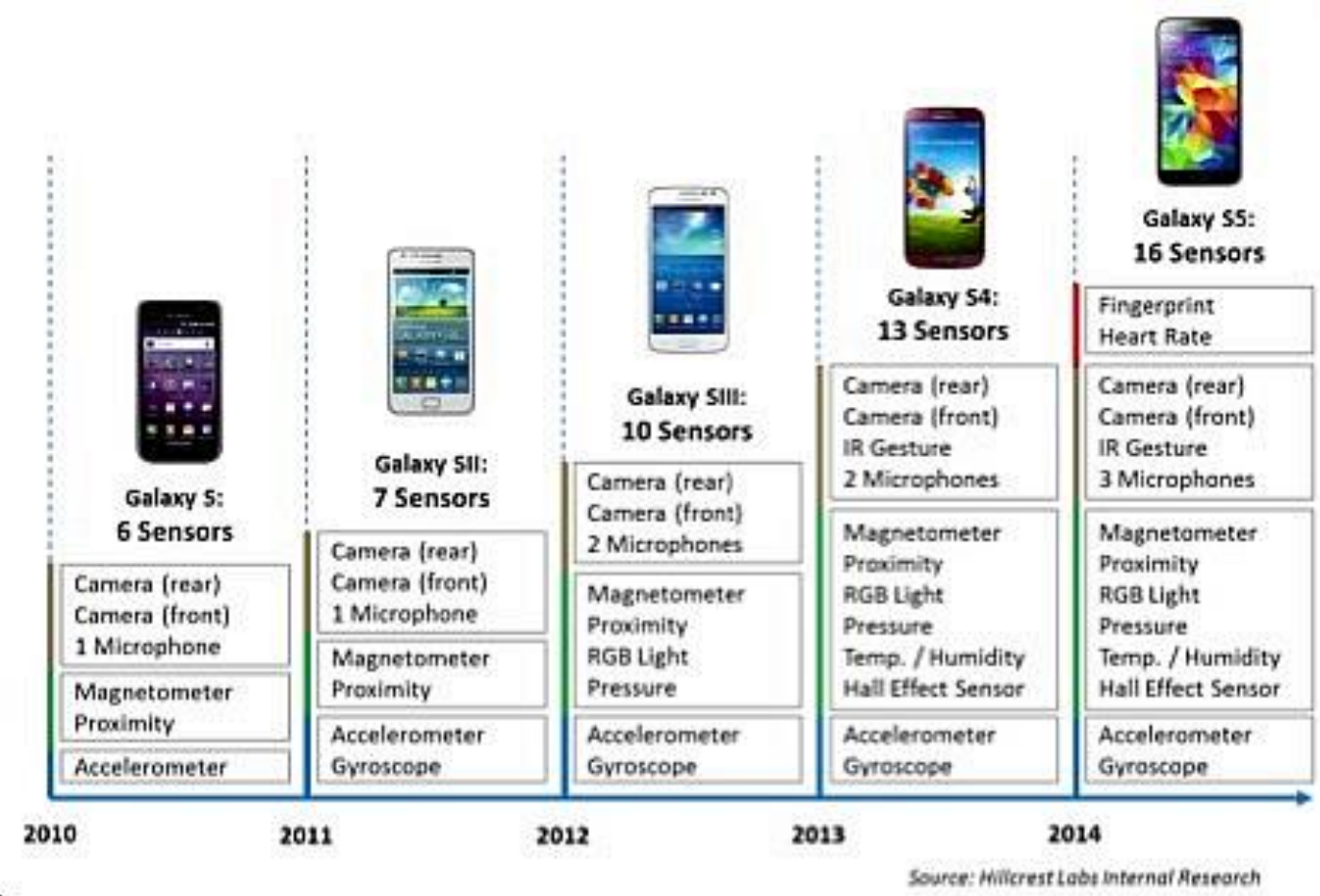

Abbildung 14: Sensoren der Produktreihe Samsung Galaxy $S^{111}$

Die abgebildeten Sensoren sind jedoch nur eine Auswahl, an potentiell verbauter Hardware in Smartphones. Jeder Hersteller entscheidet im Zuge der Produktentwicklung wie viele und welche konkreten Sensoren im Rahmen der Produktion tatsächlich im Endgerät verbaut werden. Dadurch variiert die hardwareseitige Ausstattung der verwendeten Sensorik von Modell zu Modell.

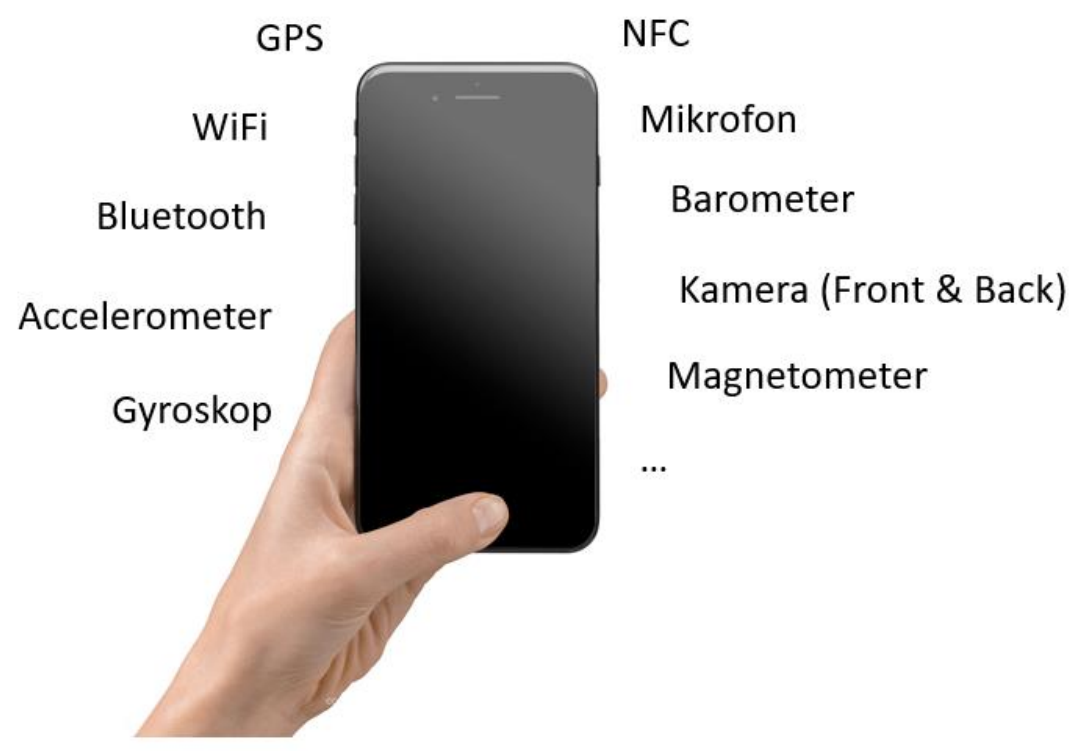

Abbildung 15: Hardwarekomponenten für die Standortbestimmung ${ }^{112}$

\footnotetext{
${ }_{111}^{11}$ Quelle: Kumar 2016.

${ }^{112}$ Quelle: Eigene Darstellung auf Basis von https://maquette.pro/product/hand-with-iphone-7s-pluspsd-mock-up/
} 
Abbildung 15 zeigt welche weiteren Hardwarekomponenten für eine Standortbestimmung Messwerte liefern könnten. Die Abbildung erhebt vor allem im Hinblick auf die gesamt verfügbare Sensorik keinen Anspruch auf Vollständigkeit, soll jedoch die Grundlage für Kapitel 5.4 - Technologien zur indoor Positionsbestimmung legen.

Im Rahmen einer stichprobenartigen Evaluation unter aktuell verfügbaren Smartphones, denen das OS Android oder iOS zu Grunde liegt, sollte die Frage der Verfügbarkeit oben genannter Sensoren geklärt werden. Als Basis für die Untersuchung diente das Online Portal „inside handy“1133 und dessen Handy-Finder ${ }^{114}$ in der Profi-Ansicht. Die verfügbare Grundmenge an Smartphones zum aktuellen Bearbeitungszeitpunkt beläuft sich, unter Berücksichtigung der genannten Präferenzen auf insgesamt 659 Stück. ${ }^{115}$ Im Hinblick auf die Fehlerbetrachtung gilt zu berücksichtigen, dass die ermittelten Treffermengen von der Korrektheit der zur Verfügung gestellten Daten durch das Portal abhängen. Zur Verschaffung eines Überblickes, über die derzeitige Marktabdeckung bezüglich der Sensorik, die in Smartphones verbaut ist, sollte die bereitgestellte Datenbasis jedoch ausreichend sein.

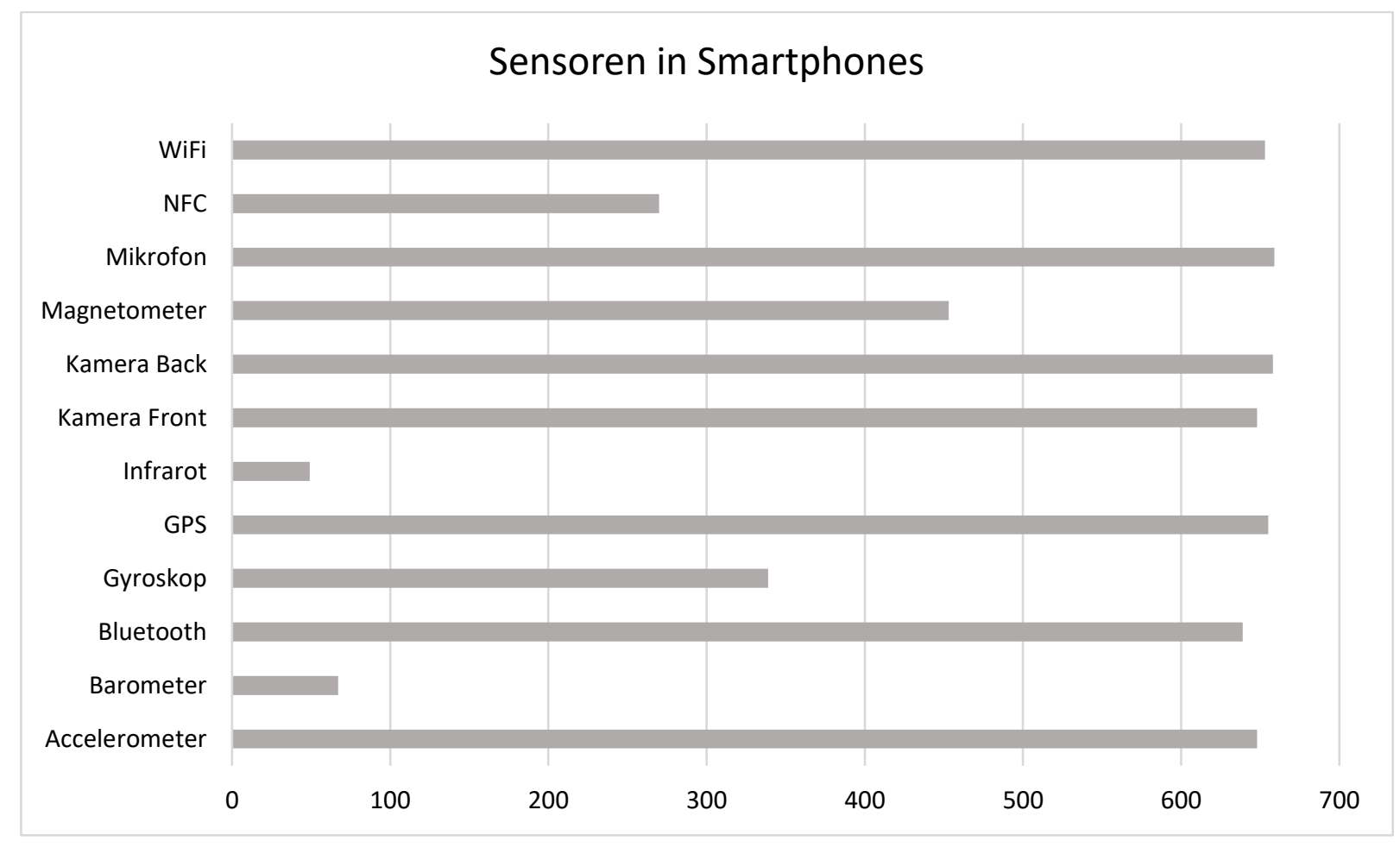

Abbildung 16: Überblick über verbaute Sensoren in Smartphones ${ }^{116}$

\footnotetext{
${ }^{113}$ Weitere Informationen unter: www.inside-handy.de

${ }^{114} \mathrm{Vgl}$. inside-intermedia GmbH 2018.

${ }^{115}$ Vgl. ebd. - mit den Filtern Verfügbarkeit: ja; Betriebssystem: Android, iOS.

${ }^{116}$ Quelle: Eigene Darstellung auf Datenbasis von www.inside-handy.de
} 
Erwartungsgemäß sind Standard-Komponenten, wie beispielsweise WiFi, in Form einer WLAN-Antenne, Mikrofon, Global Positioning System (GPS), Kamera, BT und Accelerometer in nahezu allen derzeit verfügbaren Endgeräten verbaut. Bezüglich Magnetometer, Gyroskop und NFC lässt sich sagen, dass durchschnittlich jedes zweite Gerät eine verbaute Hardwarekomponente aufweist. Exotische Schlusslichter sind das Barometer und Infrarot. Folglich können Technologien auf alleiniger Grundlage der beiden Bauteile für ein IPS nicht relevant sein.

Interessant wäre eine Untersuchung des Erscheinungsdatums der Smartphones, in Korrelation mit den drei Komponenten NFC, Magnetometer und Gyroskop. Haben beispielsweise moderne Geräte tendenziell öfter die drei Komponenten verbaut als ältere Geräte? Leider lässt die Vergleichsplattform hier keine Filtermöglichkeit zu.

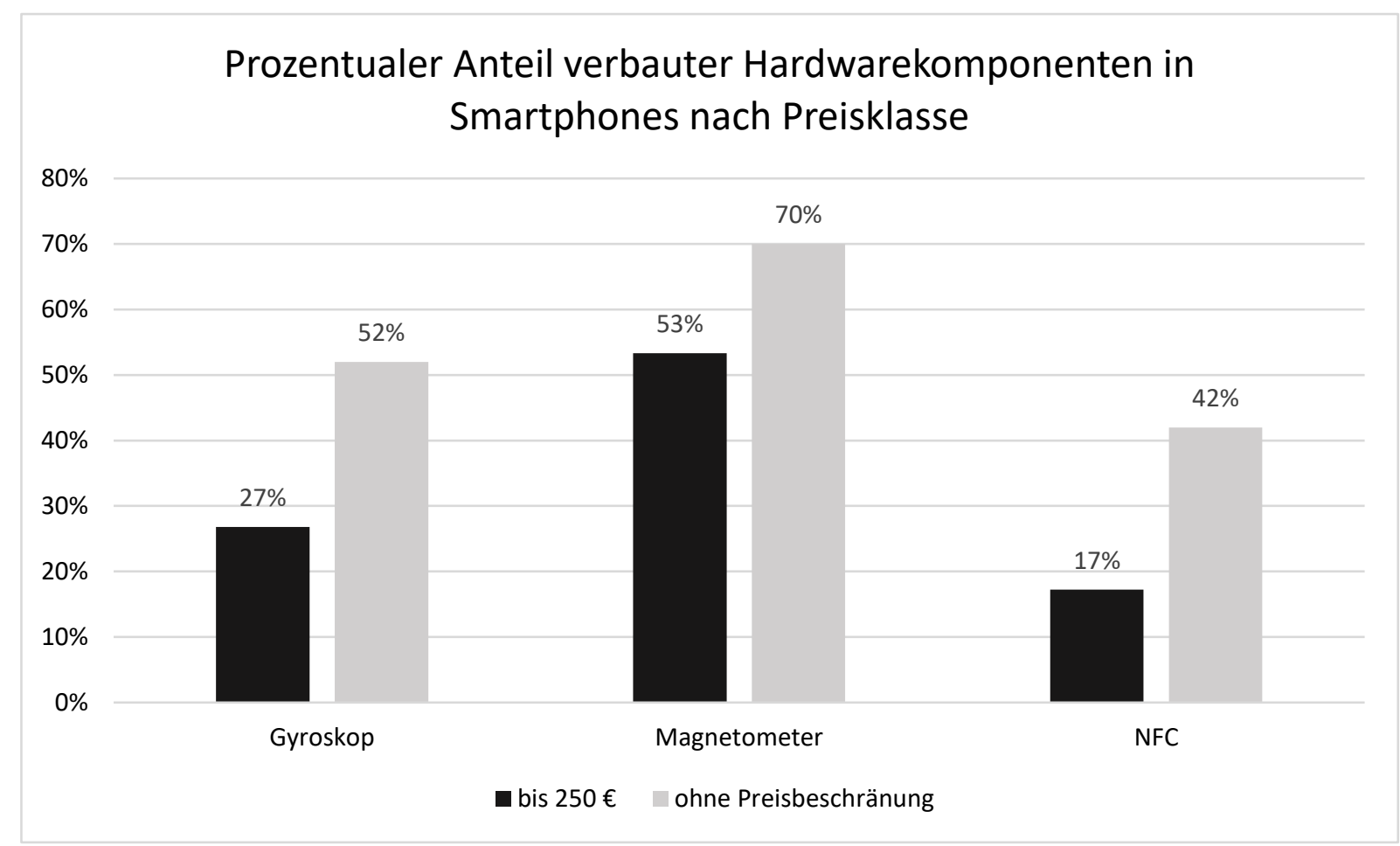

Abbildung 17: Überblick über verbaute Sensoren in Smartphones ${ }^{117}$

Einzig die Wechselwirkung zum Preis kann evaluiert werden. Im Segment bis $250 €$ unverbindliche Preisempfehlung bei Markteinführung finden sich insgesamt $302 \mathrm{Ge}-$ räte $^{118}$ der Einstiegs- und Mittelklasse. Das Säulendiagramm in Abbildung 17 zeigt deutlich, dass gerade im Hinblick auf die Integration der Hardwarekomponenten für NFC und Gyroskop der Preisfaktor für das Endgerät offensichtlich eine entscheidende

\footnotetext{
${ }^{117}$ Quelle: Eigene Darstellung auf Datenbasis von www.inside-handy.de

${ }^{118}$ Vgl. inside-intermedia GmbH 2018 - mit den Filtern Verfügbarkeit: ja; Betriebssystem: Android, iOS; Einführungspreis: $0-250 €$.
} 
Rolle spielt. Beide Sensoren weichen im Vergleich zu Smartphones ohne Preisbeschränkung um jeweils 25 Prozent ab. Es lässt sich schlussfolgern, dass Smartphones im oberen Preissegment tendenziell eine breitere Palette an Sensorik von Haus aus mitbringen. Dementsprechend ist die Sensorik bei günstigeren Produkten ein Einsparungspotential, dass die Hersteller aktiv nutzen und bewusst auf einige Bauteile verzichten. 


\section{Indoor Positioning System}

\subsection{Definition}

Um einen LBS, anbieten zu können wird ein System zur Standortbestimmung benötigt. Je nach Kontext und Anforderungsszenario ist ein geeignetes IPS oder Outdoor Positioning System (OPS) zu wählen. Aufgrund der Zielstellung der Auftraggeberin soll die Entwicklung eines IPS im Vordergrund stehen. Dies impliziert die Konzeption und Bereitstellung eines Indoor-LBS.

Sofern der Begriff IPS in seine originären Wortbestandteile zerlegt wird, bleibt eine Kombination aus den Begriffen „Indoor“, „Positioning“ und „System“ zurück. Der Begriff "Indoor" legt in diesem Kontext den Grundstein für das Anwendungsgebiet eines solchen Systems und definiert klar seine Ausrichtung. Ein IPS ist ein System, dass Objekte oder Personen innerhalb eines Gebäudes lokalisieren kann. ${ }^{119}$ Aufgrund der Fokussierung auf den Innenraum grenzt sich ein IPS eindeutig von einem OPS ab. Demnach unterscheiden sich beide Systeme im Hinblick auf ihre Anwendungsbereiche und damit verbunden divergieren auch ihre Anforderungen.

Der Begriff „Positioning“ verdeutlicht den funktionalen Charakter des Systems. Es muss demnach Verfahren und Techniken zur Standortbestimmung geben, die sich für den Aufbau eines IPS anbieten. Die Vielzahl an Möglichkeiten, hinblickend auf die verwendbaren Technologien zur Bestimmung der Positionierung in Innenräumen soll in diesem Kapitel eingehend dargestellt werden. Wie in der Einleitung bereits angedeutet wird in dem Bereich IPS aktuell viel Forschung betrieben. Beispielsweise werden immer weiter verbesserte Algorithmen zur Positionsberechnung in verschiedenen, teils speziellen Anwendungsszenarien erforscht und evaluiert. Diese ausgefeilten mathematischen Kalkulationen bedingen jedoch teilweise sehr spezifische Konstellationen der verwendeten Technologien zur Bestimmung des Standortes. Diese Arbeit grenzt sich insofern ab, als dass keine Grundlagenforschung in dem Bereich der Positionsbestimmung betrieben, sondern vielmehr ein Überblick bestehender Möglichkeiten gegeben werden soll.

\footnotetext{
${ }^{119}$ Vgl. Gu et al. 2009, S. 15.
} 
Das Wort „System“ als letzter Teil in der Wortfolge IPS verweist auf den Grad der Komplexität. Ein System ist „[e]ine Sammlung (oder eine Menge) von Elementen, die in einer bestimmten Umgebung oder in einem bestimmten Kontext eine Einheit bilden oder als Einheit aufgefasst werden “120. Ein System der Informationstechnik wiederum ist "[j]edes Gebilde, das Daten verarbeiten kann und in einem gegebenen Kontext (in einer bestimmten Umgebung) als Funktionseinheit betrachtet wird“" ${ }^{\text {121 }}$. Diese Definitionen verdeutlichen, dass ein IPS ein Zusammenspiel aus zahlreichen verschiedenen Komponenten sein muss. Eine Reduktion auf einzelne Elemente, wie beispielsweise dem Frontend steht im Hintergrund, sondern es soll vielmehr eine funktionale Einheit gebildet werden.

\subsection{Nicht-funktionale Anforderungen}

Neben den funktionalen Anforderungen, die im Kapitel 6 beschrieben werden und im Wesentlichen durch die Auftraggeberin festgelegt werden, gibt es eine Reihe von nicht-funktionalen Anforderungen, die generell an ein IPS gestellt werden können. Diese allgemeine Gültigkeit bedingt die Herauslösung aus dem Kapitel „Anforderungs- und Zielgruppenanalyse“.

„Funktionale Anforderungen beziehen sich darauf, was ein System leisten soll“"122 und können auf konkrete anwendungsspezifische Beschreibungen abstrahiert werden. So kann beschrieben werden, welche Dienste ein IPS anbieten soll, welche Eingaben entgegengenommen, Verarbeitungen durchgeführt oder Ausgaben getätigt werden. ${ }^{123}$ Darüber hinaus kann das Verhalten in bestimmten Situationen festgelegt werden, dass das System gegebenenfalls explizit nicht tun soll. ${ }^{124}$

Im Gegensatz dazu beziehen sich nicht-funktionale Anforderungen darauf, „wie das System seine Leistung erbringen soll. " ${ }^{\text {“125 }}$ Diese Anforderungen können allgemein an IPS formuliert werden und haben gleichermaßen für das zu konzipierende System Gültigkeit.

\footnotetext{
${ }^{120}$ Dierstein 2003, S. 4.

${ }^{121}$ Ebd., S. 5.

${ }^{122}$ Taentzer 2014, S. 83.

${ }^{123}$ Vgl. Ostermann, S. 15.

${ }^{124} \mathrm{Vgl}$. ebd.

${ }^{125}$ Taentzer 2014, S. 88.
} 
Aufgrund der allgemeinen Relevanz über eine Vielzahl an Systemen hinweg, sind nicht-funktionale Anforderungen komplex und umfassend. Abbildung 18 zeigt eine Basis an Anforderungen an ein IPS und wichtige technische Parameter, die bei der Entwicklung dieses Systems berücksichtigt werden können. Für den spezifischen Anwendungsfalls ist es jedoch ratsam genau abzuwägen welche der gezeigten Kriterien für ein Projekt wichtig sind und welche eine geringere Gewichtung erhalten. ${ }^{126}$

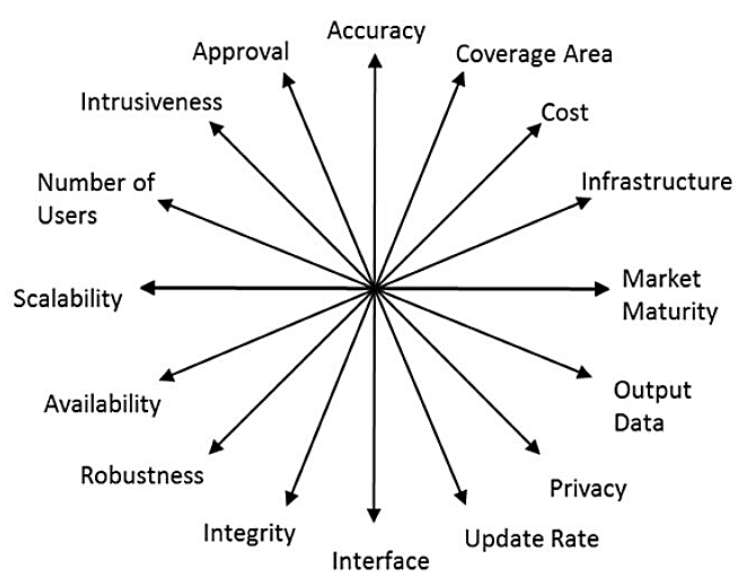

Figure 2.1 User requirements

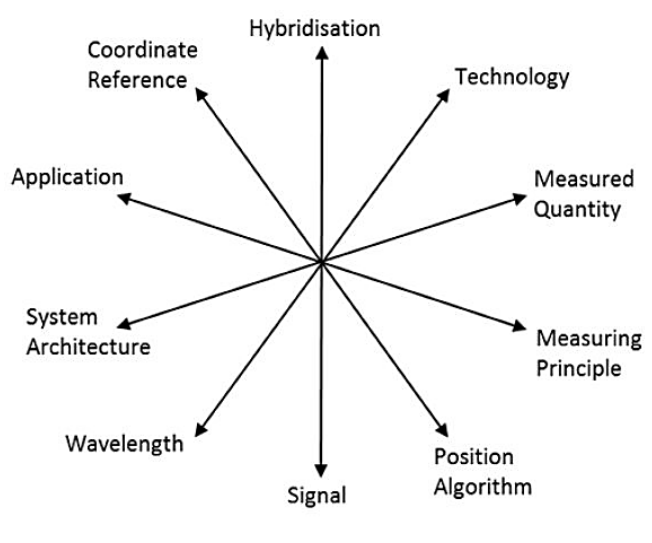

Figure 2.2 Important technical parameters being

Abbildung 18: nicht-funktionale Anforderungen und technische Parameter ${ }^{127}$

Im Zuge der Erstellung der Anforderungsanalyse und den daraus resultierenden Ergebnissen hat sich herauskristallisiert, dass einige der genannten nicht-funktionalen Anforderungen im Vergleich zu anderen von geringerer Bedeutung sind. Dies spiegelt sich, bedingt durch die Anwendungsszenarien gleichfalls in den technischen Parametern wieder. Die essentiellen Elemente sollen im Folgenden beschrieben werden.

Die Genauigkeit oder Accuracy wurde vom Joint Committee for Guides in Metrology definiert. Die Ungenauigkeit eines Systems wird über den Vergleich gemessener und erwarteter Werte bestimmt. ${ }^{128}$ Für ein IPS wird eine möglichst geringe Varianz beider Werte angestrebt, da die Genauigkeit eine der wichtigsten Anforderungen ist. ${ }^{129}$ Es ist anzumerken, dass eine hohe Genauigkeit oft nur zu Lasten anderer Kriterien realisiert werden kann und die Gewichtung immer individuell, in Bezug auf die zu realisierenden Anforderungsszenarien, abgewägt werden sollte. ${ }^{130}$

\footnotetext{
${ }^{126}$ Vgl. Mautz 2012, S. 15.

${ }^{127}$ Quelle: Ebd.

${ }^{128} \mathrm{Vgl}$. International vocabulary of metrology 2012, S. 21.

${ }^{129}$ Vgl. Liu et al. 2007, S. 1071.

${ }^{130}$ Vgl. ebd., S. 1072.
} 
Oft im Einklang mit dem Kriterium der Genauigkeit wird die Präzision oder Precision genannt. Mit dieser wird der Prozentsatz bezeichnet, mit der die vorab bestimmte Genauigkeit erwartet werden kann. ${ }^{131}$ Es kann damit als Maß für die oben genannte Varianz der gemessenen Punkte und der zugehörigen Referenzpunkte dienen. Im Einklang mit einer möglichst hohen Genauigkeit sollte demnach eine hohe Präzision der gelieferten Positionsdaten einher gehen. Im Zweifelsfall sollte der Präzision Vorzug gegenüber der Genauigkeit gegeben werden. ${ }^{132}$

Ein weiteres Element ist die Reichweite oder Coverage, dass in Bezug auf IPS eine Rolle spielt. Diese beschreibt die räumliche Ausdehnung, bei der die erfolgreiche Positionsbestimmung durch das IPS garantiert sein muss. Bezüglich der Konzeption wird empfohlen sich auf eine von drei Kategorien, Local, Scalable und Global Coverage festzulegen. ${ }^{133}$ Während Local Coverage einen kleinen, fest definierten Bereich, wie beispielsweise einen Raum oder ein Gebäude beschreibt, meint Scalable Coverage, dass das System in der Lage ist, um zusätzliche Areale erweitert zu werden. Dies kann zum Beispiel durch das Hinzufügen von weiterer Hardware erreicht werden. Eine globale Reichweite wird nur durch eine weltweite Abdeckung des Systems erreicht und ist im Sinne dieses zu konzipierenden IPS nicht zielführend. Prinzipiell gilt, je größer der potentiell abgedeckte Bereich durch das System ist, desto besser ist dies. ${ }^{134}$ Wie bereits ersichtlich ist, spielt an dieser Stelle außerdem die Skalierbarkeit oder Scalability des Systems mit hinein. Dies ist somit ein Beispiel, dass mehrere nicht-funktionale Anforderungen ineinandergreifen und sich nicht vollständig voneinander abgrenzen lassen.

Ein anderes wichtiges Kriterium sind Kosten, die im Zusammenhang mit einem IPS entstehen. Bereits im Rahmen der Konzeption sollte die Quantifizierung aller einhergehenden Faktoren sorgfältig vorgenommen werden. Faktoren, die unter anderem im Hinblick auf Kosten eine Rolle spielen sind Geld, Zeit, Raum und Energie. ${ }^{135}$ Zeit wird sowohl für Wartung, als auch für die Installation der verschiedenen Komponenten eines Systems benötigt. Dies kann sich wiederum in dem Bedarf personeller Ressourcen niederschlagen, die am besten von Anfang an mit eingeplant werden. Monetäre

\footnotetext{
${ }^{131}$ Vgl. Stephan et al. 2009, S. 202.

${ }^{132}$ Vgl. Liu et al. 2007, S. 1072.

${ }^{133}$ Vgl. Mautz 2012, S. 18.

${ }^{134}$ Vgl. Al Nuaimi und Kamel 2011, S. 186.

${ }^{135}$ Vgl. Liu et al. 2007, S. 1072.
} 
Mittel sind im Rahmen von Installation und Wartung, zum Beispiel zur Beschaffung von Hard- und Software mit zu kalkulieren. Neu aufzustellende Infrastruktur für ein IPS kann ferner einen bestimmten räumlichen Bedarf erfordern, der vorhanden sein muss. ${ }^{136}$ Darüber hinaus ist Energie ein wesentlicher Faktor. So muss berücksichtigt werden, dass verschiedene Technologien, wie zum Beispiel BLE-Beacons teilweise auf eine externe Energiequelle in Form von Akkus angewiesen sind. ${ }^{137}$ Gerade im Hinblick auf die Anforderung Kosten ist es zielführend Synergieeffekte zu nutzen. Wenn Infrastruktur, wie WLAN-Access Points (AP) für andere Zwecke angeschafft und betrieben werden, dann könnten eben diese für ein IPS genutzt werden.

Neben den bereits genannten Anforderungen spielt die Robustheit des zu entwickelnden Systems eine tragende Rolle. Diese Anforderung kann auf diverse Ebenen abstrahiert werden und geht teilweise auch mit dem Thema Sicherheit einher. Robustheit bezeichnet die Fähigkeit eines Systems, sich in Fehlerfällen oder unvorhergesehenen Ereignissen akzeptabel zu Verhalten. ${ }^{138}$ Unter anderem muss das System gegen unbefugten Zugriff, Diebstahl, physischen Schaden oder Manipulationsversuchen gesichert sein. ${ }^{139}$ Des Weiteren geht damit einher, dass das IPS mit einer gewissen Fehlertoleranz ausgestattet ist und Routinen bereithält, die beispielsweise den Fall abfangen, dass keine relevanten oder nur ungenaue Daten zur Positionsbestimmung empfangen werden können. ${ }^{140}$

Wie bereits erwähnt spielt der Aspekt der Sicherheit in verschiedenen Kontexten zu anderen Anforderungen eine Rolle. Dies ist ebenfalls in Bezug auf den Aspekt der Privatsphäre oder Privacy der Fall. Gerade im Zuge, der vor kurzem in Kraft getretenen Datenschutz-Grundverordnung ist der Schutz sensibler, personenbezogener Daten ernst zu nehmen. Das System sollte im Hinblick auf Privacy by Design so gestaltet sein, das nur zwingend benötigte Daten erhoben und gespeichert werden. ${ }^{141}$ Zum einen ist bezüglich dieses Kriteriums die generelle Absicherung des Systems, wie im Zuge der Robustheit beschrieben, gemeint und zum anderen soll ebenfalls bereits die Architektur des IPS die Privatsphäre des Nutzers sicherstellen. So besteht ein Unterschied, ob ein Frontend Daten der Positionsbestimmung an das Backend weiter reicht

\footnotetext{
${ }^{136} \mathrm{Vgl.} \mathrm{Mautz} \mathrm{2012,} \mathrm{S.} 20$.

${ }^{137} \mathrm{Vgl}$. Gu et al. 2009, S. 18.

${ }^{138}$ Vgl. Fernandez et al. 2005, S. 333.

${ }^{139}$ Vgl. Mautz 2012, S. 16.

${ }^{140} \mathrm{Vgl}$. Liu et al. 2007, S. 1072.

${ }^{141}$ Vgl. Weitere Informationen liefert Art. 25 DSGVO.
} 
und dieser anhand der übermittelten Daten den Standort berechnet, oder ob dies bereits im Frontend geschieht und das Backend die erhobenen Daten des Anwenders nicht gesendet bekommt. Im zweiten Fall ist architektonisch sichergestellt, dass nur das Frontend personenbezogene Daten erhebt und verarbeitet, womit prinzipiell eine höhere Privatsphäre des Nutzers einhergeht.

Neben den genannten nicht-funktionalen Anforderungen gibt es noch zahlreiche weitere, die im Rahmen einer Konzeptionierung und Entwicklung eines Systems berücksichtigt werden müssen. An dieser Stelle ist keine Vollständigkeit beabsichtigt, sondern vielmehr sollen die genannten Charakteristika als Ausschnitt in Betracht gezogen werden und mit den spezifischen Anforderungen der Auftraggeberin korrespondieren.

\subsection{Techniken zur Indoor-Positionsbestimmung}

Techniken der Indoor-Positionsbestimmung dienen bei IPS dazu den Standort des Anwenders zu bestimmen bzw. einzuschätzen. Es existieren eine Reihe von Algorithmen und Techniken, um Messdaten im Sinne von Peilungs-, Entfernungs- oder Abstandsinformationen basierend auf Signalmessungen oder Signaleigenschaften zu erhalten. ${ }^{142}$ Das IPS nutzt die Algorithmen, um aufgezeichnete Signaleigenschaften in Abstände und Winkel zu übersetzen, aus denen wiederum auf die aktuelle Position des Nutzers eines IPS geschlossen werden kann. ${ }^{143}$ Die meisten der bisher bekannten Techniken, Algorithmen und Komponenten zur Positionsbestimmung sind nicht neu, sondern sind in Teilen von OPS übernommen, da hier die selben Signaleigenschaften zu Grunde liegen. ${ }^{144}$ Jedoch unterscheiden sich Rahmenbedingungen und Anforderungen von IPS und OPS, sodass in den letzten Jahren zunehmend Forschungsaufwand im Hinblick auf Indoor-Positionsbestimmung betrieben worden ist.

\footnotetext{
${ }^{142}$ Vgl. Amundson und Koutsoukos 2009, S. $242 \mathrm{f}$.

${ }^{143}$ Vgl. Al Nuaimi und Kamel 2011, S. 186.

${ }^{144}$ Vgl. Sakpere et al. 2017, S. 150.
} 


\subsubsection{Positionsbestimmung anhand von Signaleigenschaften}

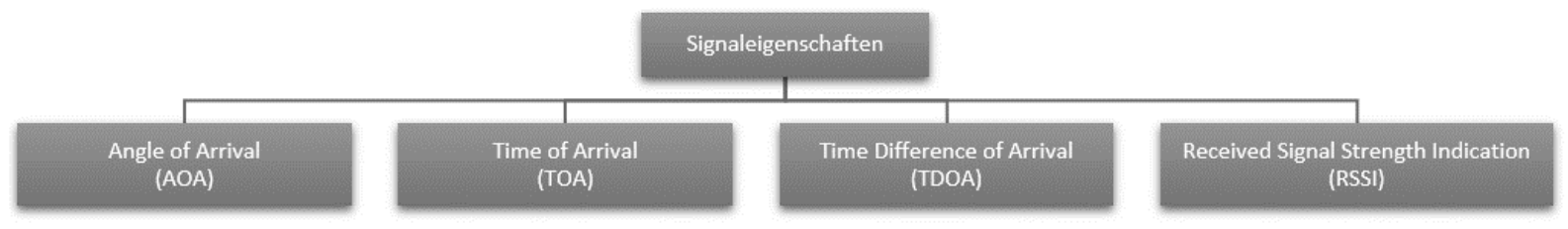

Abbildung 19: Techniken zur Positionsbestimmung anhand der Signaleigenschaften ${ }^{145}$

Signale weisen unterschiedliche Attribute bzw. Eigenschaften auf, mit denen es möglich ist den Standort von Objekten zu messen. ${ }^{146} \mathrm{Im}$ Wesentlichen können über die vier in Abbildung 19 gezeigten Methoden relevante Daten für eine Positionsbestimmung anhand der Signaleigenschaften gesammelt werden. Diese Charakteristika können von einem Sender emittiert oder von einem Empfänger bei Ankunft des Signales gemessen werden. ${ }^{147}$

Die Angle of Arrival (AOA)-Methode nutzt die Winkel der eintreffenden Signale des Zielgerätes zu den Empfängern, um daraus die Position des Objektes zu bestimmen. ${ }^{148}$ Im zweidimensionalen Raum reichen bereits zwei Empfänger bzw. Referenzpunkte, um über diese Methode den Standort zu schätzen. ${ }^{149}$ Für eine Indoor-Positionsbestimmung über mehrere Räume hinweg ist diese Technik jedoch kritisch zu sehen, da "Störfaktoren«, wie die Reflektion des Signals an den Wänden die Messergebnisse derart verzerren kann, dass diese nahezu unbrauchbar werden. ${ }^{150}$

Während Positionsdaten mit AOA auf Winkeln basieren, beruht die Time of Arrival (TOA)-Methode hauptsächlich auf Entfernungen. Bei Verwendung der Technik TOA wird die Zeit gemessen, die ein Signal, dass von einem festen Sender ausgestrahlt wird benötigt, um bei dem Empfänger anzukommen. ${ }^{151}$ Wichtig ist zu wissen, wann das Signal vom Sender emittiert worden ist. Darüber hinaus müssen die verwendeten Uhren bei Sender und Empfänger zur akkuraten zeitlichen Messung exakt synchronisiert sein. ${ }^{152}$ Problematisch ist in diesem Hinblick, dass sich Funksignale nahezu mit Schallgeschwindigkeit ausbreiten, was es schwierig macht diese zeitlich präzise zu

\footnotetext{
${ }^{145}$ Quelle: Eigene Darstellung.

${ }^{146}$ Vgl. Al Nuaimi und Kamel 2011, S. 186.

${ }^{147}$ Vgl. ebd.

${ }^{148}$ Vgl. Amundson und Koutsoukos 2009, S. 242.

${ }^{149}$ Vgl. Sakpere et al. 2017, S. 152.

${ }^{150}$ Vgl. Farid et al. 2013, S. 3.

${ }^{151}$ Vgl. Al Nuaimi und Kamel 2011, S. 186.

${ }^{152}$ Vgl. Amundson und Koutsoukos 2009, S. 242.
} 
messen. ${ }^{153}$ Kleinste Abweichungen im Bereich von Nanosekunden können die Messergebnisse sehr stark verzerren und eine Positionsbestimmung ungenau machen. ${ }^{154}$ Des Weiteren ist eine Verwendung in der Praxis, aufgrund der Anfälligkeit gegenüber »Störfaktoren« bedenklich. ${ }^{155}$

Wie die Positionierungstechnik TOA ist auch Time Difference of Arrival (TDOA) eine Methode, die auf der Entfernung basiert. Die Idee hinter TDOA ist, die relative Positionierung eines mobilen Senders anhand der Zeitdifferenz des Signals zu verschiedenen Referenzpunkten zu bestimmen. ${ }^{156}$ Mit anderen Worten misst diese Methode die Differenz von TOA-Messungen an zwei verschiedenen Sensoren. ${ }^{157}$ Dies macht wiederum das Wissen, um den exakten Startzeitpunkt des zu lokalisierenden Senders obsolet, womit lediglich die verschiedenen Empfänger des Signals synchronisiert sein müssen. ${ }^{158}$

Die letzte zu betrachtende Methode ist die Received Signal Strength Indication (RSSI). Diese Technik ist nur für Funksignale relevant, da sie die Stärke der empfangenen Signale auswertet und basierend auf dieser die Entfernung zwischen Sender und Empfänger bestimmt. ${ }^{159}$ RSSI schätzt anhand der Reduktion bzw. dem Verlust der Signalstärke, beispielsweise aufgrund der Ausbreitung des Signals, die Entfernung. ${ }^{160}$ Je näher sich ein zu lokalisierendes Objekt zum Beispiel an einem WiFi-AP befindet, umso höher wird die empfangene Signalstärke sein, worauf wiederum auf die Entfernung geschlossen werden kann. Es ist zu beachten, dass diese Methode anfällig für "Störfaktoren« ist, was wiederum ihre Genauigkeit einschränkt. ${ }^{161}$ Dafür lässt sich meist bestehende Infrastruktur für diese Technik der Messung von Positionsdaten nutzen, womit zumeist eine Kostenersparnis verbunden ist. ${ }^{162}$

Abbildung 20 aggregiert die vorgestellten Methoden zur Positionsbestimmung anhand der Signaleigenschaften. Sie verdeutlicht, dass situativ abzuwägen ist, welche der Techniken im Hinblick auf die Signaleigenschaften für ein Anwendungsszenario

\footnotetext{
${ }^{153}$ Vgl. Amundson und Koutsoukos 2009, S. 243.

${ }^{154}$ Vgl. Mautz 2012, S. 30.

${ }^{155}$ Vgl. Brás et al. 2012, S. 2.

${ }^{156}$ Vgl. Liu et al. 2007, S. 1069.

${ }^{157}$ Vgl. Sakpere et al. 2017, S. 153.

${ }^{158}$ Vgl. Amundson und Koutsoukos 2009, S. 243.

${ }^{159}$ Vgl. Sakpere et al. 2017, S. 154.

${ }^{160}$ Vgl. Liu et al. 2007, S. 1069.

${ }^{161}$ Vgl. Brás et al. 2012, S. 4.

${ }^{162}$ Vgl. ebd.
} 
zu wählen ist. Wie im folgenden Kapitel beschrieben wird, ist erst eine Kombination mit verschiedenen passenden Algorithmen für eine effiziente Standortbestimmung des zu lokalisierenden Objektes zielführend.

\begin{tabular}{|c|c|c|c|}
\hline Signal property & Measurement metric & Pros & Cons \\
\hline Angle of Arrival (AOA) & Angle-based & High accuracy at room level & $\begin{array}{l}\text { Complex, expensive and low } \\
\text { accuracy at wide coverage }\end{array}$ \\
\hline Time of Arrival (TOA) & Distance-based & High accuracy & Complex and expensive \\
\hline $\begin{array}{l}\text { Time Difference of Arrival } \\
\text { (TDOA) }\end{array}$ & Distance-based & High accuracy & Expensive \\
\hline $\begin{array}{l}\text { Received Signal Strength } \\
\text { Indication (RSSI) }\end{array}$ & Signal-based (RSS) & Low cost & Medium accuracy \\
\hline
\end{tabular}

Abbildung 20: Zusammenfassung der betrachteten Techniken ${ }^{163}$

\subsubsection{Algorithmen zur Positionsbestimmung}

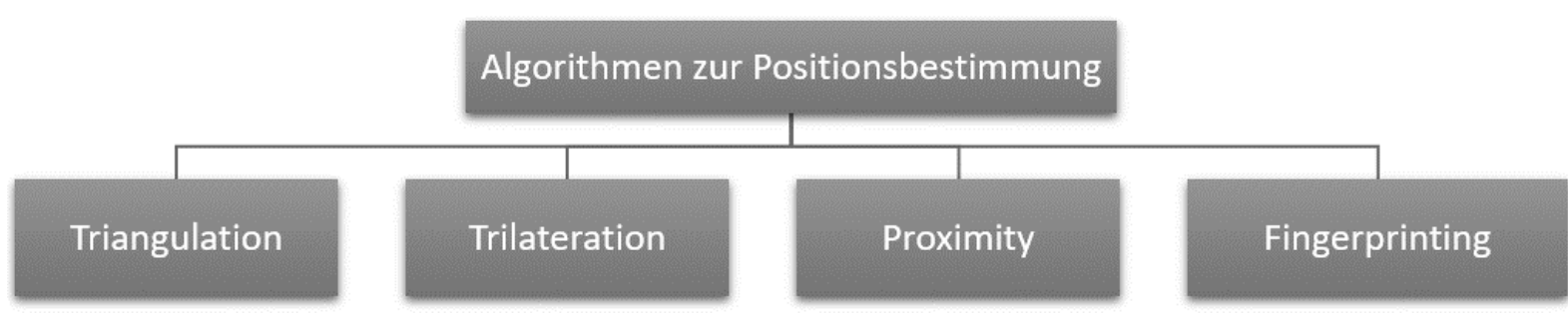

Abbildung 21: Algorithmen zur Positionsbestimmung ${ }^{164}$

Positionsbestimmungsalgorithmen definieren, wie genau die Position des Zielobjektes kalkuliert wird. ${ }^{165}$ Folglich übersetzen diese Algorithmen die aufgezeichneten Signaleigenschaften in Entfernungen und Winkel, mit denen anschließend die Berechnungen durchgeführt werden können. ${ }^{166}$ Das heißt die Algorithmen verarbeiten die Attribute bzw. Eigenschaften der Signale und liefern eine Position des zu lokalisierenden Objektes zurück. Die wesentlichen Algorithmen zur Standortbestimmung sind Triangulation, Trilateration, Proximity und Fingerprinting. ${ }^{167}$ Eigentlich lässt sich die Triangulation in ihre zwei Derivate Lateration (Trilateration) und Angulation aufteilen. ${ }^{168} \mathrm{Im}$ Sinne der Übersichtlichkeit sind diese beiden Verfahren jedoch auf zwei separate Bezeichnungen aufgeteilt worden, da diese in der Form in der Literatur zu finden sind.

\footnotetext{
${ }^{163}$ Quelle: Sakpere et al. 2017, S. 154.

${ }^{164}$ Quelle: Eigene Darstellung.

${ }^{165} \mathrm{Vgl}$. Gu et al. 2009, S. 17.

${ }^{166}$ Vgl. Sakpere et al. 2017, S. 155.

${ }^{167}$ Vgl. Mautz 2012, S. $31 f$.

${ }^{168}$ Vgl. Liu et al. 2007, S. 1068.
} 
Triangulation oder Angulation nutzt die geometrischen Eigenschaften von Dreiecken, um eine Positionsbestimmung durchzuführen, indem Winkelberechnungen relativ zu mindestens zwei bekannten Referenzpunkten durchgeführt werden. ${ }^{169}$ Darüber hinaus wird zur Kalkulation eine Länge, beispielsweise zwischen den beiden Referenzpunkten benötigt. ${ }^{170}$ Im Zuge des Algorithmus wird die AOA-Methode verarbeitet, indem der Schnittpunkt von Winkellinien der Referenzpunkte zur Standortbestimmung des zu lokalisierenden Objektes genutzt wird. ${ }^{171}$ Je mehr Referenzpunkte bekannt sind und zur Berechnung des Schnittpunktes verwendet werden, desto präziser ist der Algorithmus. $^{172}$ Im Zuge der Umsetzung eines IPS mit Triangulation ist zu berücksichtigen, dass ein System damit rasch komplex wird und meist erhöhte finanzielle Mittel erfordert.

Der Algorithmus Trilateration oder Lateration bedient sich ebenfalls der geometrischen Eigenschaften von Dreiecken, um die Position des Zielobjektes zu bestimmen. ${ }^{173}$ Jedoch werden an dieser Stelle keine Winkelberechnungen durchgeführt, sondern es werden die Abstände zu mehreren verschiedenen Referenzpunkten zur Standortbestimmung herangezogen. ${ }^{174}$ In einem zweidimensionalen Raum sind hierbei drei Längenmessungen von bekannten Referenzpunkten notwendig. ${ }^{175}$ Wenn sich je ein Signal kreisförmig von den drei genannten Referenzpunkten ausbreiten würde, dann würde der Schnittpunkt aller drei Kreise der Standort des Zielobjektes sein. ${ }^{176}$ Die Längen vom Referenzpunkt zum Zielobjekt, sozusagen die Radien der gedachten Kreise, werden mittels der Abschwächung des ausgestrahlten Signals berechnet. ${ }^{177}$ Für die Umsetzung der Trilateration werden die Signaleigenschaften TOA und TDOA, mit ihren spezifischen Vor- und Nachteilen verwendet. ${ }^{178}$ Es ist zu beachten, dass »Störfaktoren« durch beispielsweise Umwelteinflüsse die Genauigkeit des Algorithmus beeinflussen können. ${ }^{179}$

\footnotetext{
${ }^{169}$ Vgl. Liu et al. 2007, S. 1070.

${ }^{170}$ Vgl. Amundson und Koutsoukos 2009, S. 244.

${ }^{171}$ Vgl. Liu et al. 2007, S. 1070.

${ }^{172}$ Vgl. Gu et al. 2009, S. 16.

${ }^{173}$ Vgl. Sakpere et al. 2017, S. 155.

${ }^{174}$ Vgl. Liu et al. 2007, S. 1068.

${ }^{175}$ Vgl. Amundson und Koutsoukos 2009, S. 244.

${ }^{176}$ Vgl. ebd.

${ }^{177}$ Vgl. Sakpere et al. 2017, S. 155.

${ }^{178}$ Vgl. Liu et al. 2007, S. $1068 f$.

${ }^{179}$ Vgl. Sakpere et al. 2017, S. 156.
} 
Ein gänzlich anderes Verfahren als Triangulation und Trilateration verwendet Proximity. Hierbei geht es nicht darum eine absolute oder relative Position zurück zu liefern, sondern lediglich Informationen über die Nähe zum tatsächlichen Standort anzubieten. ${ }^{180}$ Voraussetzung ist eine beliebige Anzahl an Detektoren, deren Position bekannt ist, um den Standort des zu lokalisierenden Objektes annäherungsweise zu schätzen. ${ }^{181} \mathrm{Im}$ Hinblick auf die Signaleigenschaften verwendet Proximity das Attribut RSSI. $^{182}$ Je nach Anforderung an das IPS kann die Anzahl an Detektoren variiert werden. Je größer deren Anzahl, desto genauer und fein granularer kann der Algorithmus arbeiten. Sollte das Objekt von mehr als einem Detektor gefunden werden, dann wird derjenige mit der größten Signalstärke zur Lokalisierung verwendet. ${ }^{183}$

Vorteil dieser Methode ist, dass Sie einfach und darüber hinaus meist kosteneffizient zu implementieren ist. ${ }^{184}$ Des Weiteren können für diesen Algorithmus unterschiedlichste Technologien verwendet werden. ${ }^{185}$ Ein simples Anwendungsbeispiel könnte die Beantwortung der Frage sein, ob sich ein Zielobjekt in einem Quadranten befindet oder nicht.

Der letzte zu betrachtende Algorithmus zur Bestimmung der Position ist Fingerprinting oder auch Scene Analysis genannt. Dieses Berechnungsverfahren operiert unabhängig von gemessenen Winkeln oder Längen. ${ }^{186}$ Ein Fingerprint ist eine eindeutige Signatur oder eine Sammlung von Signaturen, die einen Standort von einem anderen unterscheidet bzw. unterscheiden. ${ }^{187}$ Der Algorithmus kann die RSSI-Methode verwenden, um die Standortbestimmung durchzuführen und bietet sich vor allem bezüglich der Verwendung in drahtlosen bzw. auf Funkwellen basierten Netzwerken an. ${ }^{188}$ Es ist hingegen möglich, dass auch andere Daten, außer RSSI-Werte als Fingerprints verwendet werden. Solange die Messwerte konsistent und gleichbleibend sind können diese als Signaturen genutzt werden.

Zumeist unterscheidet sich der Ablauf in zwei verschiedene Phasen, eine OfflinePhase, die dem Training des Systems und der Kalibrierung dient und einer Online-

\footnotetext{
${ }^{180}$ Vgl. Gu et al. 2009, S. 16.

${ }^{181}$ Vgl. Liu et al. 2007, S. 1071.

${ }^{182}$ Vgl. Sakpere et al. 2017, S. 156.

${ }^{183}$ Vgl. Al Nuaimi und Kamel 2011, S. 186.

${ }^{184}$ Vgl. Farid et al. 2013, S. 3.

${ }^{185}$ Vgl. Liu et al. 2007, S. 1071.

${ }^{186} \mathrm{Vgl}$. Sakpere et al. 2017, S. 156.

${ }^{187}$ Vgl. Al Nuaimi und Kamel 2011, S. 186.

${ }^{188}$ Vgl. Mautz 2012, S. $57 f$.
} 
Phase, die zur Bestimmung der Position fungiert. ${ }^{189}$ Während der Offline-Phase werden Fingerprints gesammelt und einem bestimmten Standort zugeordnet. Nach Möglichkeit sollen von einem Standort mehrere verschiedene räumliche Stellen erfasst und damit verbunden auch differierende Signaturen hinterlegt werden, um die spätere Matching-Phase zu erleichtern. ${ }^{190}$ Dies kann zum Beispiel mit einer Unterteilung des Standortes in einzelne Raster erfolgen. Jedem der Raster wird anschließend eine Liste von RSSI-Tupeln zugeordnet. ${ }^{191}$

In der Online-Phase werden die gesammelten Fingerprints mit den aktuell vorliegenden Daten verglichen. Der Standort wird aufgrund der größtmöglichen Übereinstimmungsrate zwischen den Datensets ermittelt. ${ }^{192}$ Dieses Matching-Verfahren wiederum kann mithilfe von unterschiedlichen Algorithmen, beispielsweise Entscheidungsbäumen oder dem k-Nearest-Neighbor-Algorithmus gelöst werden. ${ }^{193}$ Fingerprinting ist während der Trainingsphase mit einem initialen Aufwand zur Erfassung der Datenbasis verbunden. Darüber hinaus ist die Flexibilität in Bezug auf räumliche Veränderungen eingeschränkt. ${ }^{194}$ Je nach angeforderter Genauigkeit kann der Positionsbestimmungsprozess eine erhöhte Verarbeitungszeit und Rechenkapazität benötigen. ${ }^{195}$ Vorteilhaft kann jedoch sein, dass bestehende Infrastruktur genutzt und somit im Vergleich zur Triangulation sowie Trilateration eine Reduktion der Kosten und Komplexität erreicht werden kann. ${ }^{196}$

\subsection{Technologien zur indoor Positionsbestimmung}

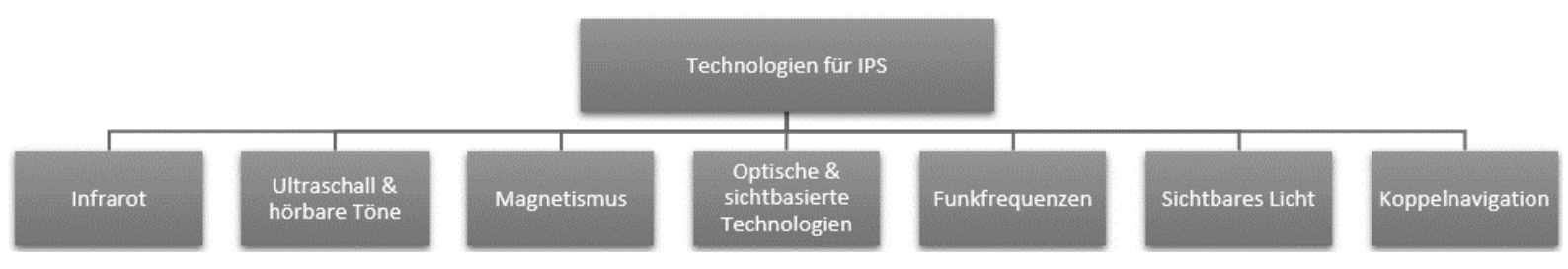

Abbildung 22: Klassifikation von Technologien für $\operatorname{IPS}^{197}$

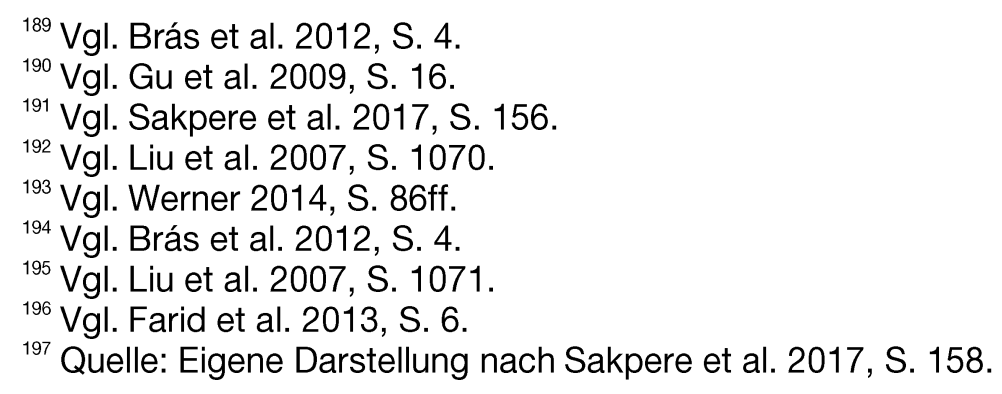


Neben den beschrieben Methoden und Algorithmen besteht ein IPS aus einer oder mehreren technologischen Komponenten, die unter anderem Messungen der relevanten Daten überhaupt erst ermöglichen. Es ist anzumerken, dass zu jeder der klassifizierten Technologien eigene Abhandlungen verfasst werden könnten. Des Weiteren beschäftigen sich diverse Forschungsarbeiten mit der Konzeption und Evaluation von IPS, die nur auf einer der gezeigten Unterkategorien basieren. Im Rahmen der vorliegenden Arbeit soll ein selektiver Überblick mit aggregierten Vor- und Nachteilen aus der aktuellen Forschung als Stand der Technik geliefert werden, der vor allem bezüglich der Verwendung von Sensoriken in Smartphones abgegrenzt wird. Daneben soll dieses Kapitel die Grundlage für die Evaluation in Teil 7 der Arbeit bilden.

Es sei bereits vorab erwähnt, dass Schwächen einzelner Technologien durch eine Kombination mehrerer Techniken kompensiert werden können und im Rahmen einer „Sensorfusion“ oder „Hybrid-Lösung“ die Stärken mehrerer Technologien gebündelt werden können. Dies ist auch bei zahlreichen IPS im Rahmen diverser Forschungsarbeiten konzipiert und umgesetzt worden sowie ebenfalls im Rahmen dieser Arbeit, je nach Anforderungen, vorstellbar.

\subsubsection{Positionierung auf Basis von Infrarot}

Eines der ersten mobilen Positionsbestimmungssysteme basierte auf Infrarot und nennt sich „Active Badge“ ${ }^{\text {(198 }}$. Grundlage dieses Systems ist, dass der Nutzer ein kleines elektronisches Gerät bei sich trägt, dass in periodischen Abständen ein Infrarotsignal emittiert. ${ }^{199}$ Dieses Signal wiederum wird von einem Sensornetzwerk, dass in die Infrastruktur des Gebäudes integriert ist ausgewertet und bestimmt die Position des Nutzers. ${ }^{200}$

Vorteilhaft ist, dass Infrarotstrahlung vom menschlichen Auge nicht wahrgenommen und somit unsichtbar eingesetzt werden kann. ${ }^{201}$ Nachteilig ist jedoch, dass wie in

\footnotetext{
${ }^{198}$ Active Badge ist ein Projekt, dass zwischen 1989 und 1992 an den AT\&T Laboratories in Cambridge konzipiert und umgesetzt worden ist. Wesentlich an der Entwicklung sind Andy Hopper, Roy Want, Andy Harter, Tom Blackie, Mark Chopping, Damian Gilmurray, und Frazer Bennett beteiligt gewesen. Weitere Informationen zu dem Projekt sind unter: https://www.cl.cam.ac.uk/research/dtg/attarchive/ab.html zu finden.

${ }^{199} \mathrm{Vgl}$. Want et al. 1992, S. $92 f$.

${ }^{200}$ Vgl. ebd., S. 93ff.

${ }^{201}$ Vgl. Mautz 2012, S. 42.
} 
Kapitel 4.4 aufgezeigt, nur noch ein verschwindend geringer Anteil der aktuellen Smartphones diese Technologie als Schnittstelle beinhaltet. Ein zusätzliches elektronisches Gerät ist in der heutigen Zeit aus Nutzersicht nicht mehr zielführend.

Darüber hinaus kann die Anschaffung zusätzlicher Hardware, vor allem basierend auf einer hohen Skalierbarkeit, den Faktor Kosten negativ beeinflussen. ${ }^{202}$ Neben dem Kostenfaktor wird diese Technologie darüber hinaus durch die Notwendigkeit des Bestehens einer Sichtlinie zwischen Sender und Empfänger limitiert. ${ }^{203}$

\subsubsection{Positionierung auf Basis von Ultraschall \& hörbaren Tönen}

Ultraschall und hörbare Töne sind sich im Prinzip als Technologie sehr ähnlich. Primärer Unterschied ist die Wahrnehmung der Technologien durch den Menschen. Im Bereich des Ultraschalls gibt es ähnlich, wie für Infrarot-Systeme eine Vielzahl an bestehenden und erforschten Systemen, die eine unterschiedliche Herangehensweise an die Positionsbestimmung ermöglichen.

Stellvertretend sei das IPS „Active Bat ${ }^{\star 204}$ genannt und betrachtet. Prinzipiell funktioniert dies ähnlich, wie das unter Infrarot beschriebene System „Active Badge“ und basiert auf Trilateration kombiniert mit TOA. ${ }^{205}$ Ein Sender strahlt ein Ultraschallsignal aus, dass wiederum von einem Sensornetzwerk ausgewertet wird. ${ }^{206}$ Bei einem aktiven System wie diesem, in dem der Sender ein, für den Menschen nicht hörbares Ultraschallsignal aussendet, muss das Endgerät überhaupt dazu in der Lage sein, diesen Ton zu emittieren. Die Unterstützung dieser Frequenzen ist bei Smartphones tendenziell fragwürdig. ${ }^{207}$

Im Gegensatz zu Ultraschall nutzen hörbare Töne für den Menschen akustisch wahrnehmbare Schallwellen, um den Anwender innerhalb von Gebäuden zu lokalisieren. Gerade im Umfeld von Bibliotheken, die häufig als Lernort fungieren und in denen eine Atmosphäre der Ruhe zum ungestörten Arbeiten sowie Lernen herrscht, sind hörbare

\footnotetext{
${ }^{202}$ Vgl. Mautz 2012, S. 42.

${ }^{203}$ Vgl. Sakpere et al. 2017, S. 159.

${ }^{204}$ Active Bat ist ein Projekt, das ebenfalls an den AT\&T Laboratories in Cambridge entwickelt worden ist. Es ist in der Zeit zwischen 1997 und 2001 von verschiedenen Forschungsmitgliedern konzeptioniert und betreut worden. Die wesentlichen Akteure innerhalb des Projektes waren Andy Ward, Peter Steggles, Rupert Curwen, Paul Webster, Mike Addlesee, Joe Newman, Paul Osborn, Steve Hodges.

${ }^{205} \mathrm{Vgl}$. AT\&T Laboratories Cambridge 2005.

${ }^{206}$ Vgl. ebd.

${ }^{207}$ Vgl. Lazik und Rowe 2012, S. 11.
} 
Töne störend für die Nutzer und somit nicht zielführend. Eine prinzipielle Umsetzung eines solchen IPS mit einem Smartphone demonstriert das System „Beep“ ${ }^{\text {208 }}$.

Ein generelles Problem birgt jedoch, wie bei TOA üblich, die Voraussetzung der Synchronität von Sender und Empfänger sowie die Beeinträchtigung der Messergebnisse durch andere Geräusche. ${ }^{209}$ Nachteilig wirkt sich darüber hinaus die potentielle Komplexität und damit verbunden, die nicht zu unterschätzenden Kosten des Systems aus. ${ }^{210}$ Sowohl aktive, als auch passive Systeme leiden unter "Störfaktoren«. Es können zum Beispiel Schallwellen an der Infrastruktur von Innenräumen gebrochen oder reflektiert werden. ${ }^{211}$ Vorteilhaft ist hingegen, dass eine Genauigkeit im Bereich von Zentimetern erzielt werden kann. ${ }^{212}$

\subsubsection{Positionierung auf Basis von Magnetismus}

Diese Methode der Positionsbestimmung basiert auf der Nutzung von künstlich erzeugten magnetischen Feldern bzw. dem Magnetfeld der Erde. ${ }^{213}$ Ein solches IPS nutzt magnetische Signale innerhalb eines magnetischen Feldes, um eine Positionsbestimmung durchzuführen. ${ }^{214}$ Neben der Nutzung von künstlich erzeugten Magnetfelder, beispielsweise durch starke Magneten oder elektromagnetische Spulen, ist die Verwendung des Magnetfeldes der Erde ein gängiges Verfahren für IPS. ${ }^{215}$ Das natürliche Magnetfeld der Erde wird innerhalb eines Gebäudes durch Anomalien, hervorgerufen durch Metalle und Elektrogeräte, wie Stahlträger oder Aufzüge, beeinflusst. ${ }^{216}$ Unter der Voraussetzung, dass sich das magnetische Feld in Innenräumen, eben unterbrochen durch künstliche und natürliche Anomalien, nicht ändert, kann der Fingerprinting Algorithmus eindeutige magnetische Signaturen erfassen. ${ }^{217}$

Wie bei Fingerprinting Algorithmen üblich, können nach einer Trainingsphase die Signaturen zur Standortbestimmung miteinander verglichen werden. Ein Nachteil bei der

\footnotetext{
${ }^{208} \mathrm{Vgl}$. Mandal et al. 2005.

${ }^{209} \mathrm{Vgl}$. Angelis et al. 2017, S. 4.

${ }^{210}$ Vgl. Sakpere et al. 2017, S. 163.

${ }^{211} \mathrm{Vgl}$. Gu et al. 2009, S. 22.

${ }^{212}$ Vgl. Mautz 2012, S. 56.

${ }^{213} \mathrm{Vgl}$. Li et al. 2012, S. $1 f$.

${ }^{214} \mathrm{Vgl}$. Sakpere et al. 2017, S. 165.

${ }^{215}$ Vgl. Mautz 2012, S. $100 f f$.

${ }^{216} \mathrm{Vgl}$. Kim et al. 2012, S. 1.

${ }^{217}$ Vgl. Carrillo et al. 2015, S. $17170 f$.
} 
Sensornutzung des Smartphones als Frontend für die passive Fingerprinting Methode ist, dass die magnetischen Sensoren im Idealfall korrekt kalibriert sein müssen, um akkurate und damit verwertbare Messwerte zu liefern. ${ }^{218}$ Andernfalls bedarf es ausgefeilter Algorithmen, um eben diese Messungenauigkeiten zu kompensieren. ${ }^{219}$ Gerade bei einem Szenario mit einer Vielzahl an unterschiedlichen Geräten kann es fraglich sein, ob eine vorherige Kalibrierung immer praktikabel ist.

In Kombination mit verschiedenen Berechnungsverfahren wird Magnetic-Fingerprinting häufig mit anderen Technologien zur Standortbestimmung, wie Koppelnavigation oder WLAN, verknüpft. ${ }^{220}$ Dies führt zu einer genaueren Positionsbestimmung, erhöht jedoch die Komplexität der Implementierung.

Darüber hinaus kann die Bauweise von Smartphones im Hinblick auf elektromagnetische Komponenten eine Ungenauigkeit der Messwerte hervorrufen. ${ }^{221}$ Auch muss in Betracht gezogen werden, dass einige Positionen innerhalb des Gebäudes Bezug nehmend auf ihre magnetische Signatur einander stark ähneln oder, dass sich magnetische Feldstärken ändern können, beispielsweise durch das Aufstellen elektromagnetischer Geräte. 222

Im Gegensatz dazu ist von Vorteil, dass diese Technologie vollkommen frei von infrastrukturellen Veränderungen oder Erweiterungen ist. ${ }^{223}$ Des Weiteren befindet sich, wie in Kapitel 4.4 aufgezeigt, in einem Großteil der Smartphones ein Magnetometer. Abschließend sei erwähnt, dass es sich um eine energieeffiziente Technologie handelt, da keine zusätzliche Infrastruktur benötigt wird und die Nutzung des Magnetfeldmessers im Smartphone wenig Akku-Leistung in Anspruch nimmt. ${ }^{224}$

\subsubsection{Positionierung auf Basis von optischen \& sichtbasierten Technologien}

Optische oder sichtbasierte IPS sind Systeme, bei denen der Standort des Zielobjektes innerhalb eines Gebäudes unter Verwendung einer Kamera bestimmt wird. ${ }^{225}$

\footnotetext{
${ }^{218}$ Vgl. Shahidi und Valaee 2015, S. $1 f$.

${ }^{219}$ Vgl. Shahidi und Valaee 2015, S. 4f \& Carrillo et al. 2015, S. $17174 f$.

${ }^{220}$ Vgl. Kim et al. 2012, S. 5 \& Liu et al. $2017-2017$, S. 129.

${ }^{221}$ Vgl. Shao et al. 2016, S. 2

${ }^{222}$ Vgl. Carrillo et al. 2015, S. 17169.

${ }^{223}$ Vgl. ebd., S. 17191.

${ }^{224}$ Vgl. Shao et al. 2016, S. 1

${ }^{225}$ Vgl. Sakpere et al. 2017, S. 167.
} 
Unterschieden wird diese Technologie zum einen in statische, im Raum aufgestellte Kameras, die sich bewegende Objekte in Bildern erkennen und bestimmen können. ${ }^{226}$ Zum anderen in Systeme, die den Standort eines mobilen optisch Sensors, wie zum Beispiel einer Smartphonekamera, ermitteln. ${ }^{227}$

Aufgrund der Verquickung zum Smartphone als Frontend soll im Rahmen der Arbeit, in Bezug auf optische IPS, der Fokus auf der zweiten Variante liegen und näher betrachtet werden. Hierbei werden künstliche Markierungen verwendet, um den Standort des Zielobjektes zu bestimmen. ${ }^{228}$ Die Markierungen sind überwiegend feststehende Objekte, die ein bestimmtes Muster aufweisen. ${ }^{229}$ Zum Beispiel können diese »optoelektronisch lesbaren Codes«, wie 1D-Codes beispielsweise EAN-Barcodes, 2D-Codes, wie QR-Codes, Aztec-Codes oder ähnliche sein.

Die Kamera des Smartphones erfasst die Muster und wertet die darin codierten Informationen aus. ${ }^{230}$ Je nach Architektur des IPS bestimmt anschließend die Softwareanwendung im Server oder im Frontend die Position des Nutzers. Diese Technologie bietet sich insbesondere für Pull-Dienste eines LBS an. Wenn auf Markierungen in genannter Form verzichtet werden soll, dann kann ein aufgenommenes Bild hinsichtlich seines RGB-Farbraumes analysiert und mit hinterlegten Signaturen, im Rahmen eines Fingerprinting Algorithmus, verglichen werden. ${ }^{231}$ Eine andere Möglichkeit Markierungen in einer künstlichen Form zu vermeiden, die sofort durch den Nutzer als solche erkannt werden ist, vorhandenen Text als Kennzeichnung für einen Standort zu verwenden. Beispielsweise könnten bereits bestehende Beschriftungen, die vom Smartphone des Nutzers mittels Texterkennung erfasst werden, zur Positionsbestimmung ausgewertet werden. Denkbar wäre es, bestimmte eindeutige Schlüsselwörter einer konkreten Position im Raum zuzuordnen.

Vorteilhaft bei Verwendung eines optischen bzw. sichtbasierten IPS ist, dass jedes derzeit verfügbare Smartphone eine Kamera integriert hat, wie in Kapitel 4.4 ersichtlich ist. Darüber hinaus sind solche Systeme, aufgrund der minimalen Komplexität und des niedrigen Materialaufwandes meist sehr kosteneffizient zu implementieren. Dies

\footnotetext{
${ }^{226}$ Vgl. Mautz 2012, S. 34.

${ }^{227} \mathrm{Vgl}$. ebd.

${ }^{228}$ Vgl. Mautz 2012, S. 37.

${ }^{229} \mathrm{Vgl}$. ebd.

${ }^{230}$ Vgl. Sakpere et al. 2017, S. 168.

${ }^{231} \mathrm{Vgl}$. Jiao et al. 2016, S. 658.
} 
gilt auch im Hinblick auf die Energieeffizienz. Die Genauigkeit eines solchen Systems ist ebenfalls als sehr hoch einzuschätzen. ${ }^{232}$

Nachteilig können sich externe Faktoren wie beispielsweise die Lichtverhältnisse oder keine klare Sichtlinie in Räumen auswirken. ${ }^{233}$ Darüber hinaus hängt die korrekte Erkennungsrate häufig von der Qualität der verwendeten Smartphonekamera und deren Auflösungsrate $a b .^{234}$ Eine Echtzeitverfolgung der Positionierung des Nutzers, wie bei anderen Technologien ist, zumindest mit der beschriebenen Variante eines optischen IPS, nicht realisierbar. ${ }^{235}$ Dies kommt jedoch der Privatsphäre des Anwenders zugute, da dieser meist aktiv bestimmt, wann eine Positionsbestimmung erfolgen soll.

\subsubsection{Positionierung auf Basis von Funkfrequenzen}

IPS, die auf Funkfrequenzen beruhen, bestimmen den Standort mittels unterschiedlicher Funksignale und deren emittierender Infrastruktur. ${ }^{236}$ Bezüglich der verwendbaren Algorithmen zur Positionsbestimmung ist diese Technologie sehr flexibel und ermöglicht es mit allen in Kapitel 5.3.2 vorgestellten Berechnungsverfahren kombiniert zu werden. ${ }^{237}$ Abhängig von der jeweiligen Unterkategorie eignet sich ein bestimmter Algorithmus jeweils besser zur Standortbestimmung.

Radio Frequency IPS unterteilen sich in sechs Unterkategorien, mit jeweils eigenen Charakteristika sowie spezifischen Vor- und Nachteilen. Die Unterkategorien sind BT, »Ultra Wideband«, Wireless Sensor Network, WLAN, Radio-Frequency Identification (RFID), NFC und »Zigbee «. ${ }^{238}$ In diesem Kapitel werden jedoch nur die für ein Smartphone relevanten Unterkategorien näher betrachtet. Dies sind BT, WLAN und NFC. Die verbleibenden Technologien sind teilweise nur mit zusätzlicher Hardware für ein Smartphone messbar und damit nicht Gegenstand dieser Arbeit. Vorweg soll generell erwähnt werden, dass bei der Nutzung von Funksignalen Wände oder andere Hindernisse bis zu einem gewissen Grad durchdrungen werden. ${ }^{239}$ Diese Tatsache kann für

\footnotetext{
${ }^{232}$ Vgl. Puertolas-Montaez et al. 2013, S. 46.

${ }^{233}$ Vgl. Mautz 2012, S. 37.

${ }^{234}$ Vgl. Nowicki et al. 2016, S. $159 f$.

${ }^{235}$ Vgl. Sakpere et al. 2017, S. 168.

${ }^{236}$ Vgl. ebd., S. 170.

${ }^{237}$ Vgl. Gu et al. 2009, S. 17.

${ }^{238}$ Vgl. Sakpere et al. 2017, S. 170 \& Mautz 2012, S. 83.

${ }^{239}$ Vgl. Farid et al. 2013, S. 5.
} 
ein IPS sowohl ein Vor-, als auch ein Nachteil sein. Zum einen kann sich die erhöhte Reichweite vorteilhaft auf den potentiell abzudeckenden Bereich auswirken. Zum anderen können sich Signale, aufgrund von »Störfaktoren«, beispielsweise mangels Abschirmung, überlagern und eine korrekte Standortbestimmung erschweren.

BT als erste zu betrachtende Unterkategorie ist ein drahtloser Industriestandard, der für den Datenaustausch über kurze Distanzen gedacht ist und somit in Wireless Personal Area Network genutzt wird. ${ }^{240}$ Hierbei wird das $2.4 \mathrm{GHz}$ Frequenzband, wie bei WLAN teilweise auch, zu Kommunikation zwischen Sender und Empfänger verwendet. ${ }^{241}$ Im Hinblick auf IPS, vor allem im Zusammenspiel mit einem Smartphone als Frontend werden häufig BLE-Beacons zur Positionsbestimmung verwendet.

BLE ist in Version 4.0 der BT Core Specification eingeführt worden und ergänzt den BT-Standard, um eine stromsparende Komponente. ${ }^{242}$ Hauptsächlich wird die Reichweite des emittierten Signals reduziert, um den Akku des Senders zu schonen. ${ }^{24} \mathrm{Im}$ Vergleich zu einem gewöhnlichen BT-Sender weist ein BLE-Beacon einen bis zu 10fach niedrigeren Stromverbrauch auf. ${ }^{244}$ Die angesprochenen Beacons sind hierbei kleine Sender, zum Teil nur von der Größe einer Streichholzschachtel, die via BLE ihren Unique Identifier (ID) und andere Informationen emittieren. ${ }^{245}$

Die Produktpalette an Beacons ist mannigfaltig. Apple und Google haben mit ihren Standards iBeacon ${ }^{246}$ und Eddystone ${ }^{247}$ jeweils verschiedene Technologien als Basis für BLE-Beacons entwickelt. Firmen wie Estimote ${ }^{248}$ oder Kontakt.io ${ }^{249}$ vertreiben verschiedenste Beacons, die auf den beiden genannten Technologien basieren können. Vereinfacht beschrieben überträgt der Beacon seine Identifikation in Form des Unique ID bzw. der Universal Unique ID in den Raum, die dann wiederum von einem Smartphone empfangen wird. ${ }^{250}$ Nun muss der ausgelesenen ID noch eine Position im Raum zugeordnet werden. Idealerweise übernimmt ein Backend die Funktion der Verwaltung und Sicherung dieser Verknüpfung. Es kann nun abhängig von der Architektur

\footnotetext{
${ }^{240}$ Vgl. Schnabel.

${ }^{241}$ Vgl. Taskin 2017, S. 60.

${ }^{242}$ Vgl. Noertjahyana et al. 2017 - 2017, S. 186.

${ }^{243}$ Vgl. ebd.

${ }^{244}$ Vgl. Taskin 2017, S. 60.

${ }^{245}$ Vgl. Strobel 2015.

${ }^{246}$ Vgl. Apple Inc. 2018b.

${ }^{247}$ Vgl. Google LLC 2018d.

${ }^{248}$ Vgl. Estimote, Inc. 2018.

${ }^{249}$ Vgl. Kontakt.io, Inc. 2018.

${ }^{250}$ Vgl. Noertjahyana et al. 2017 - 2017, S. 187.
} 
des IPS sein, ob die Standortbestimmung ausschließlich im Frontend stattfindet oder Teile des Prozesses auf das Backend ausgelagert werden. Auch die verwendete Technik und der Algorithmus zur Standortermittlung sind vom IPS abhängig.

Vorteilhaft ist, dass sich BT durch eine hohe Sicherheit vor beispielsweise Manipulation, sowie kompakte Abmaße der Sender auszeichnet. ${ }^{251}$ Wichtig zu wissen ist, dass Hersteller für ihre Endgeräte selbstständig auswählen, welchen BT-Standard sie integrieren wollen. ${ }^{252}$ Dies gilt sowohl für Hersteller von Smartphones, als auch von Beacons. Wenn bestimmte Endgeräte unterschiedliche Standards integriert haben, dann kann es sein, dass diese sich Gegenseitig nicht erkennen. Wenn zum Beispiel ein Smartphone keine BT-Standard 4.0 integriert hat, dann kann es auch keine BLE-Beacons erkennen. Auch die Geschwindigkeit der Erkennung kann unterschiedlich lange Zeitspannen in Anspruch nehmen. ${ }^{253}$ Eine mögliche Ursache könnte hier die Verwendung unterschiedlicher Standards von BT, aber, wie in Kapitel 3.3 beschrieben, auch das Zusammenspiel unterschiedlichster Komponenten sein.

Von Nachteil ist des Weiteren, dass immer zusätzliche Hardware in Form von Beacons benötigt wird, die zum einen initiale und zum anderen fortlaufende Kosten, im Hinblick auf die Wartung, generieren. Beispielsweise muss bei der Verwendung externer Sender regelmäßig ein Batteriewechsel stattfinden, damit keiner der Beacons nach einiger Zeit ausfällt. Bei einer Vielzahl zusätzlich installierter Geräte erhöht sich dadurch der Wartungsaufwand, sowohl in Form von Personal-, als auch Materialkosten, immens. Nachteilig ist gleichwohl, dass der Batteriestatus der Beacons überwacht werden sollte, um einen verlässlichen Indikator für deren Einsatzbereitschaft zu ermitteln.

WLAN bezeichnet einen industriellen Standard der IEEE-802.11-Familie, die als Norm die Kommunikation in Funknetzwerken beschreibt. ${ }^{254} \mathrm{Im}$ Unterschied zu BT kann diese Technologie neben dem 2.4 GHz- parallel das $5 \mathrm{GHz}-$ Frequenzband nutzen. Das $5 \mathrm{GHz}$-Frequenzband arbeitet mit Super-Hochfrequenzwellen, die kürzer als die Ultra-Hochfrequenzwellen des 2.4 GHz-Bandes sind. Folglich strahlen die Wellen des 2.4 GHz-Bandes weiter als das $5 \mathrm{GHz}$-Pendant, dafür breiten sich diese wiederum

\footnotetext{
${ }^{251}$ Vgl. Mautz 2012, S. 84.

${ }^{252} \mathrm{Vgl}$. Schnabel.

${ }^{253}$ Vgl. Sakpere et al. 2017, S. $170 f$ \& vgl. Farid et al. 2013, S. 5.

${ }^{254}$ Vgl. IEEE Std 802.11ah-2016 (Amendment to IEEE Std 802.11-2016, as amended by IEEE Std 802.11ai-2016) 2017.
} 
schneller aus. ${ }^{255}$ Häufig verwenden WLAN-AP's noch das $2.4 \mathrm{GHz}-$ Frequenzband und leiden dadurch möglicherweise an »Störfaktoren«, wie Interferenzen. ${ }^{256}$

Typischerweise kann WLAN eine Distanz von bis zu 100 Metern, je nach einwirkenden "Störfaktoren«, abdecken und hat damit eine größere potenzielle Reichweite als BT und NFC. ${ }^{257}$ Vorteilhaft ist, dass meist die bereits bestehende Infrastruktur, in Form von WLAN-AP's, verwendet werden kann. ${ }^{258}$ Bezüglich der Kosten wirkt sich dies für ein IPS positiv aus. Oft werden WLAN-AP's generell in regelmäßigen Intervallen gewartet und generieren demnach keine zusätzlichen Wartungskosten.

Identisch zu BT kann das Smartphone als Empfänger der Funksignale fungieren. Auch hier kann je nach Architektur der Standort direkt im Frontend bestimmt werden, oder zentral auf einem Server. Der Algorithmus zur Standortbestimmung kann, wie bereits einleitend erwähnt frei gewählt werden, jedoch ist RSSI und damit Fingerprinting oder Proximity im Allgemeinen das gängige Mittel der Wahl. ${ }^{259}$

IPS, die auf WLAN basieren benötigen zur Standortbestimmung keine direkte Sichtlinie. ${ }^{260}$ Allerdings wird WLAN, wie generell auf Funkwellen basierte Technologie, von externen »Störfaktoren« wie Abschwächung, Absorption, Reflektion, Zerstreuung, Brechung und Beugung des Signals beeinträchtigt. ${ }^{261}$

Im Gegensatz zu den bisher vorgestellten Funk-Technologien benötigt NFC keinen aktiven Sender, sondern stellt eine passive Variation dar und bietet sich damit vor allem für LBS-Pull-Dienste an. ${ }^{262}$ NFC ist ein auf RFID basierender internationaler Übertragungsstandard zum kontaktlosen Austausch von Daten mittels elektromagnetischer Induktion über kurze Strecken von wenigen Zentimetern. ${ }^{263}$ Die Technologie benutzt den Hochfrequenz-Bereich bei $13.56 \mathrm{MHz}$ und ermöglicht Datenraten bis zu $424 \mathrm{kbit} / \mathrm{s}^{264}$ Vorab ist anzumerken, dass diese Technologie momentan einen enormen Aufschwung, durch die verstärkte Nutzung im bargeldlosen Zahlungsverkehr, erhält. $^{265}$

\footnotetext{
${ }^{255} \mathrm{Vgl}$. Fischer 2017.

${ }^{256}$ Vgl. Farid et al. 2013, S. 7.

${ }^{257}$ Vgl. Mautz 2012, S. 57.

${ }^{258} \mathrm{Vgl}$. Gu et al. 2009, S. 19.

${ }^{259} \mathrm{Vgl}$. ebd.

${ }^{260}$ Vgl. Farid et al. 2013, S. 6.

${ }^{261}$ Vgl. Puussaar 2014, S. 25.

${ }^{262}$ Vgl. Ozdenizci et al. 2015, S. 7575.

${ }^{263}$ Vgl. DATACOM Buchverlag GmbH 2014.

${ }^{264} \mathrm{Vgl}$. Ozdenizci et al. 2015, S. 7577.

${ }^{265}$ Vgl. Janssen und Porteck \& Bender 2018.
} 
Im Sinne eines IPS besteht das System hauptsächlich aus drei Komponenten, einem passiven NFC-Tag, einem Lesegerät und einer Datenbank. ${ }^{266}$ Das Smartphone als Lesegerät erzeugt ein magnetisches Feld, dass den NFC-Tag mit Strom versorgt, der wiederum anschließend seine gespeicherten Daten an das Smartphone sendet. ${ }^{267}$ Diese gespeicherten Daten können mit einer Datenbank abgeglichen werden, um den Standort zu bestimmen. ${ }^{268}$ Vorteilhaft ist folglich zum einen, dass keine externe Energiequelle benötigt wird und zum anderen sind die NFC-Tags klein, kostengünstig und weisen eine hohe Robustheit auf. ${ }^{269}$

Nachteilig wirkt sich die sehr geringe Kommunikationsreichweite zwischen Sender und Empfänger aus. Mit der geringen Distanz geht dagegen eine hohe Präzision einher. Bezüglich der NFC-Tags sollte vor allem darauf geachtet werden, dass Tags mit einem Schreibschutz ${ }^{270}$ akquiriert werden, damit die Daten nicht nachträglich manipuliert werden können. ${ }^{271}$ Wie in Kapitel 4.4 aufgezeigt, weißt aktuell nur knapp die Hälfte der derzeit verfügbaren Smartphones ein integriertes NFC-Modul auf.

\subsubsection{Positionierung auf Basis von sichtbaren Licht}

Die Technologie sichtbares Licht oder auch bekannt unter Visible Light Communication, Light-Fidelity oder Optical Wireless Communication, ist eine Datenkommunikationstechnologie, bei der Informationen mithilfe von sichtbaren Licht übertragen werden. ${ }^{272}$ Die Technologie nutzt Kompaktleuchtstoff- oder LED-Lampen, um Signale im sichtbaren Lichtspektrum zwischen $400 \mathrm{THz}$ und $800 \mathrm{THz}$ zu emittieren. ${ }^{273}$

Eine Steuereinheit moduliert das ausstrahlende Licht in dem genannten hochfrequenten Bereich, sodass Daten übermittelt werden können, ohne eine Veränderung des Lichtes für das menschliche Auge vorzunehmen. ${ }^{274}$ Das Signal wird im Anschluss von einem Bildsensor, beispielsweise der Front- oder Hauptkamera eines Smartphones,

\footnotetext{
${ }^{266}$ Vgl. Sakpere et al. 2017, S. 175.

${ }^{267}$ Vgl. Ozdenizci et al. 2015, S. 7577.

${ }^{268}$ Vgl. Sakpere et al. 2017, S. 175.

${ }^{269}$ Vgl. Mautz 2012, S. 66.

${ }^{270}$ Vgl. Stack Exchange 2011.

${ }^{271}$ Vgl. Krautz 2014, 212.

${ }^{272}$ Vgl. Gutierrez et al. 2014, S. 163.

${ }^{273}$ Vgl. Sakpere et al. 2017, S. 176.

${ }^{274}$ Vgl. Zachár et al. 2016, S. 1.
} 
verarbeitet. ${ }^{275}$ Die Aufgabe des Detektors ist es anschließend den Bitstream, der über das Licht gesendet wurde, wieder zu dekodieren. ${ }^{276}$

Je nach Architektur des IPS könnte an dieser Stelle zum Beispiel in einem bestimmten Bereich eine ID emittiert werden, die vom Frontend ausgewertet und einem Standort zugeordnet wird. ${ }^{277}$ Optional könnte die Position an ein Backend übermittelt werden. Prinzipiell kann bei dieser Technologie jeder der vorgestellten Algorithmen zur Standortbestimmung verwendet werden. ${ }^{278}$ Meist bedingt die konkrete Architektur des Systems, wie in anderen Beispielen bereits aufgezeigt, die Wahl des Algorithmus.

Vorteilhaft ist, dass nahezu jeder Innenraum durch künstliches Licht ausgeleuchtet werden muss und handelsübliche Leuchtdioden als Trägermedium dienen können. Wie bei WLAN, kann demnach bestehende Infrastruktur nachgenutzt werden und reduziert gerade die initialen Kosten, es sei denn die Leuchtmittel sind nicht für diesen Einsatz geeignet. ${ }^{279}$

Andererseits ist VLC genau in dieser Hinsicht anfällig, wenn der Detektor bei natürlicher Sonnenlichteinstrahlung oder einer verdeckten Kamera das modulierte Signal nicht eindeutig empfangen und interpretieren kann. Darüber hinaus wird im Zuge von Stromeinsparungsprozessen meist das Licht gedimmt oder tagsüber gänzlich abgeschaltet. Zusammenfassend betrachtet wird eine direkte Sichtlinie zwischen Sender und Empfänger benötigt. Bezug nehmend auf Smartphones lässt sich darüber hinaus anmerken, dass verschiedene Hardware das modulierte Licht unterschiedlich prozessieren und in Konsequenz interpretieren kann. ${ }^{280}$

\subsubsection{Positionierung auf Basis von Koppelnavigation}

Koppelnavigation bezeichnet einen Prozess, bei dem der aktuelle Standort auf Grundlage einer zuvor bekannten Positionierung zu einer bestimmten Zeit geschätzt wird. ${ }^{281}$ Die Vorhersage des Standortes basiert auf der Richtung und der Geschwindigkeit, mit

\footnotetext{
${ }^{275}$ Vgl. Chow et al. 2015, S. 2.

${ }^{276}$ Vgl. Zachár et al. 2016, S. 2.

${ }^{277}$ Vgl. Sakpere et al. 2017, S. 177.

${ }^{278}$ Vgl. ebd.

${ }^{279}$ Vgl. Sakpere et al. 2017, S. 178.

${ }^{280}$ Vgl. Chow et al. 2015, S. 3.

${ }^{281}$ Vgl. Al Nuaimi und Kamel 2011, S. 188.
} 
der sich das zu lokalisierende Objekt fortbewegt. ${ }^{282}$ Oftmals wird im Zusammenhang mit Koppelnavigation die Position von einzelnen Personen bestimmt. Man spricht in diesem Kontext in der englischen Forschungsliteratur oft von Pedestrian Dead Reckoning. Neben dieser Bezeichnung ist darüber hinaus häufig die Rede von sogenannten Inertial Navigation System, da zur Erfassung der genannten Vektoren umfangreiche Sensorikdaten benötigt und ausgewertet werden müssen.

Ein Smartphone bietet sich demnach sehr gut für diese Technologie an, da eine Vielzahl der Sensoren, wie Beschleunigungssensor, Gyroskop, Magnetometer oder Barometer vorhanden sind. Demnach wird beispielsweise der Beschleunigungssensor verwendet, um die Geschwindigkeit des zu lokalisierenden Objektes zu bestimmen oder mittels Gyroskop die eingeschlagene Richtung nachzuvollziehen. ${ }^{283}$ Folglich findet die gesamte Bestimmung der Positionierung im Frontend statt. Je nach Architektur des IPS kann an dieser Stelle wieder die Meldung an ein Backend integriert werden.

Bei den Themenpunkten Datenschutz und Privatsphäre ist die Koppelnavigation generell empfehlenswert. Ein weiterer, dadurch entstehender Synergieeffekt ist, dass keine zusätzliche Infrastruktur für die Nutzung dieser Technologie benötigt wird. ${ }^{284}$ Dies wirkt sich gleichermaßen positiv auf den Faktor Kosten aus. Es ist jedoch wichtig, dass die Sensoren im Smartphone korrekt kalibriert sind, um genaue Messwerte zu liefern.

Ein enormer Nachteil dieser Technologie ist, dass sich über eine längere Zeit eingeschlichene Fehler, zum Beispiel in Form von Messungenauigkeiten über die Zeit kumulieren und verstärken. ${ }^{285}$ Dieses Phänomen wird als „Sensor-Drift“-Effekt beschrieben und führt zu Ungenauigkeiten in der Standortbestimmung. ${ }^{286}$ Diese Abweichung kann über aufwendige Algorithmen abgeschwächt werden, erhöht im Zuge der Implementierung dafür die Komplexität des Systems. ${ }^{287}$ Des Weiteren muss der originäre Standort des Nutzers bekannt sein. ${ }^{288}$ Um dies zu ermöglichen, bedient sich die Koppelnavigation meist weiterer Technologien.

\footnotetext{
${ }^{282}$ Vgl. Harle 2013, S. 1282.

${ }^{283}$ Vgl. Sakpere et al. 2017, S. 179.

${ }^{284} \mathrm{Vgl}$. Agrawal und Toshniwal 2013 - 2013, S. 137.

${ }^{285}$ Vgl. Harle 2013, S. 1286.

${ }^{286}$ Vgl. Correa et al. 2017, S. 14

${ }^{287}$ Vgl. Harle 2013, S. 1286.

${ }^{288}$ Vgl. Štefanička et al. 2017, S. 51.
} 


\section{Anforderungs- und Zielgruppenanalyse}

\subsection{Design der Anforderungsanalyse}

Zur eindeutigen Bestimmung aller funktionalen und nichtfunktionalen Anforderungen an das zu konzipierende System, ist ein Fragebogen entworfen worden, der nach Möglichkeit sämtliche beeinflussenden Inhalte und Wünsche der Auftraggeberin Rechnung tragen soll. Die Anforderungsanalyse soll darüber hinaus für die Auftraggeberin eine Stütze sein, um eine möglichst umfangreiche gedankliche Vorstellung, ihres zukünftigen Systems zu erhalten.

Der Fragebogen soll als Leitfaden für ein Experteninterview mit den beiden Ansprechpartnern der Auftraggeberin, Frau Berg und Herrn Weiß, fungieren. Er zielt auf die realitätsnahe Erfassung aller Anforderungen und Prioritäten ab, damit im Rahmen der Konzeption ein maßgeschneiderter Entwurf für ein mit standortbasierenden Informationen agierendes System entsteht. Des Weiteren kann er anderen Bibliotheken, die ein ähnliches Vorhaben realisieren wollen, als Instrument zur Konkretisierung ihres Projekts dienen. Der vollständige Fragebogen sowie die entsprechenden Antworten der Interviewten sind dem Anhang zu entnehmen. ${ }^{289}$

Der erste Teil der Anforderungsanalyse gestaltet sich als Abfrage der inhaltlichen Rahmenbedingungen. Der Katalog an Fragen reicht von der gesamtstrategischen Einbettung des Systems, über bauliche Rahmenbedingungen, hin zu funktionalen Anforderungen an Front- und Backend. Es werden jedoch auch Themen wie Datenschutz, Präsentation des Systems im physischen Raum und konkrete abzubildende Inhalte in der späteren Anwendung abgefragt. Abgerundet werden soll der Fragebogen durch die Prioritätsanalyse im zweiten Teil. Ziel dieser Untersuchung soll ein Einblick in den Stellenwert diverser Parameter, des zu konzipierenden Systems der Auftraggeberin sein. Die Erstellung und Durchführung der Anforderungsanalyse stützt sich auf die empirische Forschungsmethodik der qualitativen Befragung. ${ }^{290}$

\footnotetext{
${ }^{289}$ Fragebogen Blanko: Pfad: ../Anhang/Anforderungsanalyse/Anforderungsanalyse.docx Protokoll Interview: Pfad: ...Anhang/Anforderungsanalyse/Interview Anforderungsanalyse.docx ${ }^{290}$ Vgl. Lang, S. 6.
} 


\subsection{Ergebnisse der Anforderungsanalyse}

Basierend auf der Auswertung der Antworten des Experteninterviews ist ein Ergebnis der Anforderungsanalyse in Form eines Anforderungskataloges, User Stories und sonstigen zu beachtenden Rahmenbedingungen entstanden. Kombiniert mit den einleitenden Beschreibungen des Philologicums in Kapitel 2 - „Portrait der Auftraggeberin“ können die Ergebnisse als Darstellung des Ist-Zustandes betrachtet werden. Im Folgenden werden die einzelnen Punkte überblicksartig abgebildet.

\subsubsection{User Stories}

Die Formulierung der User Stories lehnt sich an die Methodik der agilen Softwareentwicklung nach Scrum an. ${ }^{291}$ Dabei sollen User Stories in Alltagssprache verfasst und Softwareanforderungen in leicht verständlicher Sprache wiedergeben werden. ${ }^{292}$ Es sollen in der Regel nicht mehr als zwei Sätze zum Beschreiben einer Anforderung verwendet werden. Ein Beispiel sieht syntaktisch wie folgt aus: Als <Nutzer> möchte ich $<$ Funktion $>$, um $<$ Nutzen $>$

1. Als Student / Externer / wissenschaftlicher Mitarbeiter möchte ich mein eigenes Smartphone zur Nutzung des Systems verwenden, damit ich keine weiteren zusätzlichen Geräte benötige, sondern die Information direkt und zeitlich dann abrufen kann, wenn es die Situation erfordert.

2. Als Student / Externer / wissenschaftlicher Mitarbeiter möchte ich über aktuelle Ereignisse, wie beispielsweise einen defekten Lift, einen beginnenden Kurs, für den noch Plätze frei sind oder eine derzeit stattfindende Ausstellung informiert werden, damit ich keine zusätzlichen Informationskanäle zum Beschaffen der Informationen nutzen muss.

3. Als Student / Externer / wissenschaftlicher Mitarbeiter möchte ich Basisinformationen wie Öffnungszeiten, Telefonnummer oder Ansprechpartner des Philologicums erfahren, damit ich keine zusätzlichen Informationskanäle zum Beschaffen der Informationen nutzen muss.

\footnotetext{
${ }^{291}$ Vgl. Schwaber und Sutherland 2018.

${ }^{292}$ Vgl. Meindl 2013.
} 
4. Als Student / Externer / wissenschaftlicher Mitarbeiter möchte ich mich über die aktuellen Sitzplatzkapazitäten im Philologicum informieren, damit ich einschätzen kann, ob die Chance besteht einen freien Platz zum Arbeiten zu bekommen.

5. Als Student der Sprach- und Literaturwissenschaft / Externer / wissenschaftlicher Mitarbeiter möchte ich beim Entdecken von Kunst am Bau im Philologicum, während ich zum Betrachten davor stehen bleibe, zusätzliche Informationen auf meinem Smartphone präsentiert bekommen, damit ich ergänzende, interessante Fakten zu den Zitaten erfahre.

6. Als Student möchte ich einen Gruppenarbeitsraum direkt vor Ort reservieren und nicht beim Personal bzw. auf der Website der UB, um Studienarbeiten in diesem zu erledigen.

7. Als Student möchte ich mir die aktuellen Belegungspläne der Gruppenarbeitsräume direkt vor Ort ansehen, um zu entscheiden, ob ich den Raum kurzfristig nutzen kann, bzw. einzusehen, wie lange die aktuelle Belegung noch andauert.

8. Als Student / Externer / wissenschaftlicher Mitarbeiter möchte ich mich über die Funktionsweise der technischen Geräte im Neubau informieren, damit ich diese korrekt bedienen und verwenden kann.

9. Als Student / Externer / wissenschaftlicher Mitarbeiter möchte ich alle Informationen auch in englischer Sprache abrufen können, da Deutsch nicht meine Muttersprache ist und ich sicher sein will, alles richtig verstanden zu haben.

10. Als Student / Externer / wissenschaftlicher Mitarbeiter möchte ich die Möglichkeit haben, Inhalte wie Neuigkeiten oder Raumbuchungen von Arbeitsräumen über verschiedene Kanäle zu teilen, damit ich andere darüber informieren kann.

11. Als Student / Externer / wissenschaftlicher Mitarbeiter möchte ich auf einer Karte nachschauen, wo sich was im Philologicum befindet, damit ich mich im Gebäude schneller orientieren kann.

12. Als Student der Sprach- und Literaturwissenschaft / Externer / wissenschaftlicher Mitarbeiter möchte ich bei der Suche im Freihandbestand wissen, welche Literatur zu meinem Thema elektronisch in Form von E-Books oder Datenbanken verfügbar ist, damit ich keine separate Suche im Discovery-System der UB absetzen muss. 
13. Als Mitarbeiter möchte ich mein vertrautes Content Management System (CMS) zur Verwaltung der Inhalte des Systems verwenden, um den Arbeitsaufwand, zur vollständigen Erlernung eines neuen Systems in Grenzen zu halten.

14. Als Mitarbeiter möchte ich die anzuzeigenden Inhalte im Frontend dynamisch gestalten können, um flexibel auf Änderungen reagieren zu können.

15. Als Mitarbeiter möchte ich eine Gültigkeitsdauer für Nachrichten festlegen können, um Nutzer beispielsweise auf nur temporär gültige Angebote hinweisen zu können.

16. Als Mitarbeiter möchte ich abrufen können, welche Informationen derzeit aktiv sind und welche Informationen bereits verteilt worden sind, um stets einen Überblick über die bereitgestellten Inhalte zu haben.

17. Als Mitarbeiter möchte ich ein einfaches Tool zur Verknüpfung von Standorten mit Informationen nutzen, um steuern zu können, welche Informationen dem Nutzer an welchem Ort angezeigt werden und damit entsprechend zielgerichtet sowie flexibel agieren zu können.

\begin{tabular}{|c|c|c|c|}
\hline \multicolumn{3}{|c|}{ User Stories } \\
\hline ID & obligatorisch & ortsabhängig & \multicolumn{1}{|c|}{ Anmerkungen } \\
\hline 1 & ja & nein & \\
\hline 2 & ja & nein & \\
\hline 3 & ja & nein & \\
\hline 4 & ja & nein & Einbindung System "Platzfinder" \\
\hline 5 & ja & ja & \\
\hline 6 & ja & ja & Einbindung System "Raumreservierung" \\
\hline 7 & ja & ja & Einbindung System "Raumreservierung" \\
\hline 8 & ja & ja & \\
\hline 9 & ja & nein & \\
\hline 10 & nein & nein & \\
\hline 11 & nein & nein & Keine Echtzeitnavigation \\
\hline 12 & nein & ja & \\
\hline 13 & ja & nein & bisheriges CMS - Fiona \\
\hline 14 & ja & nein & Umsetzung im bisherigen CMS durch die UB \\
\hline 15 & ja & nein & Umsetzung im bisherigen CMS durch die UB \\
\hline 16 & ja & nein & Umsetzung im bisherigen CMS durch die UB \\
\hline 17 & ja & nein & $\begin{array}{l}\text { Notwendigkeit einer Middleware, da nicht } \\
\text { mit dem bisherigen CMS abbildbar }\end{array}$ \\
\hline
\end{tabular}

Tabelle 1: Detailangaben User Stories

Die beschriebenen User Stories sollen möglichst anschaulich darstellen, welche Anwendungsszenarien das zukünftige System bedienen können soll. Zur Priorisierung im Rahm der vorliegenden Arbeit bietet es sich an, primär ortsabhängige User Stories 
zu konzipieren und prototypisch umzusetzen. Die ortsunabhängigen Szenarien hingegen sind sekundär und werden vor allem der Vollständigkeit halber erfasst. Eine detaillierte Auflistung und Kategorisierung der User Stories sind der Tabelle 1 zu entnehmen. Im Rahmen der späteren prototypischen Umsetzung sind die User Stories, die die Merkmale „obligatorisch“ und „ortsabhängig“ erfüllen mit Vorrang umzusetzen.

\subsubsection{Anforderungskatalog}

\begin{tabular}{|c|c|c|c|}
\hline \multicolumn{4}{|c|}{ Frontend } \\
\hline ID & Typ & Beschreibung der Anforderung & Referenz \\
\hline FE 0010 & $\mathrm{~F}$ & Umsetzung als mobile Anwendung auf dem Smartphone & - \\
\hline FE 0020 & $\mathrm{~F}$ & Indoor Positionsbestimmung durchführen & FE 0010 \\
\hline FE 0030 & $\mathrm{~F}$ & Inhalte vom Backend abrufen & FE 0010 \\
\hline FE 0040 & $\mathrm{~F}$ & Integration bestehender Systeme & FE 0010 \\
\hline FE 0050 & $\mathrm{~F}$ & User Interfaces zur Interaktion und Darstellung von Informationen & FE 0010 \\
\hline FE 0060 & NF & UI in deutscher Sprache & FE 0050 \\
\hline FE 0070 & NF & UI in englischer Sprache & FE 0050 \\
\hline FE 0080 & NF & Prototyp auf Android Basis & FE 0010 \\
\hline FE 0090 & $\mathrm{~F}$ & Integration System "Platzfinder" & FE 0040 \\
\hline FE 0100 & $\mathrm{~F}$ & Integration System "Raumreservierung" & FE 0040 \\
\hline FE 0110 & NF & Darstellung statischer Inhalte & FE 0050 \\
\hline FE 0120 & NF & Darstellung dynamischer Inhalte & FE 0050 \\
\hline FE 0130 & $\mathrm{~F}$ & Inhalte mit anderen Applikationen teilen & FE 0010 \\
\hline FE 0140 & NF & UI muss sich an die Richtlinien des Corporate Design halten & FE 0050 \\
\hline FE 0150 & NF & UI soll barrierefrei sein & FE 0050 \\
\hline FE 0160 & NF & Darstellung und Aufbereitung von Text & FE 0050 \\
\hline FE 0170 & NF & Darstellung und Aufbereitung von Bildern & FE 0050 \\
\hline FE 0180 & NF & Darstellung und Aufbereitung von HTML & FE 0050 \\
\hline FE 0190 & $\mathrm{~F}$ & Verknüpfungen von Middleware abrufen & FE 0010 \\
\hline FE 0200 & NE & Auswertung strukturierter Ratenformate & FE 0030 \\
\hline FE UZUO & IN & Auswertung strukturterter vatenromate wie XIVIL oder ssuiv & FE 0190 \\
\hline FF 0210 & $\mathrm{NE}$ & Verwendung yon Standort-1R & FE 0020 \\
\hline FE UL10 & INr & verwenuung von standort-lis & FE 0190 \\
\hline FE & $\mathrm{NF}$ & Verwendung von Content-IR & FE 0030 \\
\hline 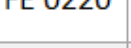 & IN & vervenuming von comtemis & FE 0190 \\
\hline & & & FE 0020 \\
\hline & & & FE 0030 \\
\hline FE 0230 & NF & Routinen und Logik zur Umsetzung von Fehlertoleranz & FE 0040 \\
\hline & & & FE 0050 \\
\hline & & & FE 0190 \\
\hline FE 0240 & NF & Abwärtskompatibilität zu Android 4.4 & FE 0080 \\
\hline FE 0250 & NF & Abwärtskompatibilität zu iOS 9.3 & FE 0010 \\
\hline
\end{tabular}

Tabelle 2: Auszug Anforderungskatalog - Frontend

Der entworfene Anforderungskatalog unterteilt sich in die jeweiligen Komponenten, des zu konzipierenden Systems, Anforderungen an die Positionsbestimmung und 
sonstige Anforderungen, die in keiner der erst genannten Bereiche untergebracht werden können. Die folgenden Tabellen sind Auszüge aus dem im Anhang befindlichen Anforderungskatalog. ${ }^{293}$ Zur Wahrung der Übersichtlichkeit sind die Spalten „Obligatorisch“ und „Anmerkung“ an dieser Stelle ausgeblendet worden. Jede Anforderung hat eine eindeutige ID, einen Typ, wobei „, $F^{\prime}$ für funktionale und „NF“ für nicht-funktionale Anforderung steht. Neben einer kurzen Beschreibung ist des Weiteren eine Referenz als Bezugspunkt angegeben.

An dieser Stelle sei angemerkt, dass das Corporate Design der UB vollständig im Anhang zu finden ist. ${ }^{294}$ Darüber hinaus ist ersichtlich, dass im Zuge der Erstellung des Anforderungskataloges bereits logische Schlussfolgerungen für die zukünftige Konzeption des Systems getroffen worden sind. Diese spiegeln sich beispielsweise in der Integration einer Middleware oder der Möglichkeit zur Auswertung strukturierter Datenformate wieder. Diese Schlussfolgerungen können als grundlegende Teilbausteine, des zu entwickelnden Konzeptes betrachtet werden und werden im Kapitel 8 entsprechend weiter ausgebaut. Abschließend erwähnt sei die Abbildung des ISTZustandes der UB, zum Beispiel ersichtlich, im Hinblick auf die Integration bestehender Systeme, wie den Platzfinder oder die Raumreservierung.

\begin{tabular}{|c|c|c|c|}
\hline \multicolumn{4}{|c|}{ Indoor Positioning } \\
\hline ID & Typ & Beschreibung der Anforderung & Referenz \\
\hline IP 0010 & $\mathrm{~F}$ & Positionsbestimmung für User Story Nr. 5 (Kunst am Bau) & FE 0020 \\
\hline IP 0020 & $\mathrm{~F}$ & $\begin{array}{l}\text { Positionsbestimmung für User Story Nr. 6, 7, 8, } 12 \\
\text { (Raumreservierungssystem, technische Geräte, Freihandbestand) }\end{array}$ & FE 0020 \\
\hline IP 0030 & NF & geringe Genauigkeit / Präzision der Positionsbestimmung & IP 0010 \\
\hline IP 0040 & NF & sehr hohe Genauigkeit / Präzision der Positionsbestimmung & IP 0020 \\
\hline IP 0050 & NF & mittlere Reichweite der Positionsbestimmung & IP 0010 \\
\hline IP 0060 & NF & geringe Reichweite der Positionsbestimmung & IP 0020 \\
\hline IP 0070 & NF & Verwendung einer Technologie die keiner baulichen Veränderungen bedarf & FE 0020 \\
\hline IP 0080 & NF & Verwendung einer Technologie die keine zusätzliche technische Infrastruktur benötigt & FE 0020 \\
\hline IP 0090 & NF & keine visuellen Veränderungen oder sonstigen Ergänzungen bezüglich der Kunst am Bau & IP 0010 \\
\hline IP 0100 & NF & Verwendung einer kostengünstigen Technologie & FE 0020 \\
\hline IP 0110 & NF & Verwendung einer wartungsarmen Technologie & FE 0020 \\
\hline IP 0120 & NF & Verwendung einer fehlertoleranten / robusten Technologie & FE 0020 \\
\hline
\end{tabular}

Tabelle 3: Auszug Anforderungskatalog - Indoor Positioning

Die Positionsbestimmung des Nutzers soll im Frontend stattfinden. Die einzelnen funktionalen und nichtfunktionalen Anforderungen sind im Sinne der Übersichtlichkeit

\footnotetext{
${ }^{293}$ Pfad: ...Anhang/Anforderungsanalyse/Anforderungskatalog.xIsx

${ }^{294}$ Pfad: ../Anhang/Corporate Design/
} 
jedoch in eine eigene Kategorie ausgelagert worden. Es ist anzumerken, dass gerade die nichtfunktionalen Anforderungen an die Bestimmung des Standortes wesentlich auf der Prioritätsanalyse des Fragebogens beruhen.

Die nichtfunktionale Anforderung IP 0090 leitet sich vor allem aus der Tatsache ab, dass aufgrund der Rechte des Architekten und der Fachplaner, wie Grafiker keine baulichen bzw. visuellen Veränderungen, vor allem an der Kunst am Bau, erlaubt sind. Gerade die Kunst am Bau ist als visuelle Einheit konzipiert sowie integriert worden und soll als diese auch auf den Betrachter wirken. Sie dient nicht der Informationsvermittlung, sondern soll vielmehr eine Atmosphäre schaffen. Somit ist es zum Beispiel nicht wünschenswert einen Aufsteller oder Display davor anzubringen, da sonst die visuelle Einheit als solches zerstört wird.

\begin{tabular}{|l|l|l|c|}
\hline \multicolumn{1}{|c|}{ ID } & Typ & \multicolumn{1}{|c|}{ Beschreibung der Anforderung } & Referenz \\
\hline MI 0010 & F & Verknüpfung zwischen Standort und Information & - \\
\hline MI 0020 & NF & persistente Sicherung der Daten & MI 0010 \\
\hline MI 0030 & NF & Bereitstellung einer API zum Abruf der Verknüpfungen & MI 0010 \\
\hline MI 0040 & NF & Nutzung eines strukturierten Datenformates wie XML oder JSON & MI 0030 \\
\hline MI 0050 & NF & Verwendung eindeutige Standort-ID & MI 0010 \\
\hline MI 0060 & NF & Verwendung eindeutige Content-ID & MI 0010 \\
\hline MI 0070 & F & User Interface zur Verwaltung der Verknüpfungen & MI 0010 \\
\hline MI 0080 & F & Abfragen bestehender Verknüpfungen & MI 0010 \\
\hline MI 0090 & F & Hinzufügen bestehender Verknüpfungen & MI 0010 \\
\hline MI 0100 & F & Löschen bestehender Verknüpfungen & MI 0010 \\
\hline MI 0110 & NF & Nutzung von Webtechnologien wie HTML, jQuery, PHP, MySQL zur Umsetzung & MI 0070 \\
\hline & & & MI 0080 \\
\hline MI 0120 & F & clientseitige Validierung der Eingaben & MI 0090 \\
& & & MI 0100 \\
\hline & & & MI 0080 \\
\hline MI 0130 & F & serverseitige Validierung der Eingabe & MI 0090 \\
& & & MI 0100 \\
\hline
\end{tabular}

Tabelle 4: Auszug Anforderungskatalog - Middleware

Wie bereits in den Hinweisen zum Frontend beschrieben hat sich die Notwendigkeit einer Middleware zur Verknüpfung des Front- und Backends im Laufe der Erstellung der Anforderungsanalyse ergeben. Genauere Ausführungen und detaillierte Angaben sind der Konzeption des Systems in Kapitel 8 zu entnehmen. Der Vollständigkeit halber sind die Anforderungen an die Middleware bereits in diesem Kapitel mit aufgeführt. 


\begin{tabular}{|r|l|l|r|}
\hline \multicolumn{1}{|c|}{ Beschreibung der Anforderung } & Referenz \\
\hline ID & Typ & - \\
\hline BE 0010 & F & Bereitstellung von Inhalten & BE 0010 \\
\hline BE 0020 & NF & Verwendung des bestehenden CMS auf Basis von Fiona & BE 0020 \\
\hline BE 0030 & NF & Bereitstellung einer API zur Auslieferung von Informationen & BE 0030 \\
\hline BE 0040 & NF & Nutzung eines strukturierten Datenformates wie XML oder JSON & BE 0020 \\
\hline BE 0050 & NF & Inhalte in deutscher Sprache vorhalten & BE 0020 \\
\hline BE 0060 & NF & Inhalte in englischer Sprache vorhalten & BE 0020 \\
\hline BE 0070 & NF & Verwendung von Content-ID & BE 0010 \\
\hline BE 0080 & F & Inhalte können mit einem Archivstatus versehen werden & BE 0010 \\
\hline BE 0090 & F & Inhalte können mit einen Gültigkeitsdatum versehen werden & \\
\hline
\end{tabular}

Tabelle 5: Auszug Anforderungskatalog - Backend

Als Backend soll nach Angaben der Auftraggeberin das bestehende CMS auf Basis von Fiona fungieren. Eine Modifizierung, für die Einbindung in das zu konzipierende System wird von Seiten der UB als realistisch und kurzfristig machbar angesehen. Gerade die Bereitstellung einer Schnittstelle zum Abruf der Inhalte ist in diesem Kontext essentiell.

\begin{tabular}{|r|l|l|r|}
\hline \multicolumn{2}{|c|}{ Sonstige } & \multicolumn{1}{c|}{ Beschreibung der Anforderung } & Referenz \\
\hline ID & \multicolumn{1}{|c|}{ Typ } & FE 0010 \\
\hline SO 0010 & F & Erstellung einer Dokumentation & MI 0010 \\
\hline SO 0030 & NF & Erstellung des Quellcodes als Open Source und Veröffentlichung & FE 0010 \\
\hline
\end{tabular}

Tabelle 6: Auszug Anforderungskatalog - Sonstige

Die sonstigen Anforderungen dienen im Grunde zur Sicherstellung der Nachhaltigkeit und dem Open Source Gedanken des Systems.

\subsubsection{Sonstige zu beachtende Rahmenbindungen}

Das Nutzungskonzept des Philologicums sieht vor dieses in drei verschiedene Zonen aufzuteilen. Die Zonen richten sich nach der darin erwarteten zukünftigen Lautstärke. Es wird in eine laute und leise sowie eine "Silentium“-Zone unterschieden. Der Silentium Bereich sind hierbei die Lesesäle, in denen absolute Stille herrschen soll. Dies ist vor allem für eine eventuelle Verwendung akustischer Technologien zu beachten.

Im Hinblick auf die Kunst am Bau sei aufgeführt, dass es sich immer um Zitate und Sprüche der Literaturgeschichte in diversen Sprachen handelt. Alle Texte sind auf Glasscheiben angebracht und weisen dieselbe Schriftfarbe auf. Lediglich die 
typographische Gestaltung, beispielsweise Schriftgröße oder Abstände, können von Zitat zu Zitat variieren. Wichtig anzumerken ist, dass es sich nicht um Bilder handeln wird.

Die von der Auftraggeberin zur Verfügung gestellten Bau- und Etagenpläne des Philologicums sind in Auszügen dem Anhang zu entnehmen. ${ }^{295}$ Sie beinhalten unter anderen die konkrete Positionierung technischer Geräte im Neubau sowie die Verortung der Kunst am Bau. ${ }^{296}$ Darüber hinaus sind alle Zitate der Kunst am Bau ebenfalls im Anhang aufgeführt. ${ }^{297}$

\subsection{Zielgruppenanalyse}

Im Rahmen der Konzeption wird die umfangreiche quantitative Befragung potentieller Zielgruppen als zu komplex und zeitintensiv eingeschätzt, deswegen dienen statistische Daten aus diversen Studien als Grundlage für die Zielgruppenanalyse. Basierend auf den ausgewerteten Daten und den, in der Anforderungsanalyse entwickelten User Stories sollen als Resultat separate Personas für jede einzelne Zielgruppe entstehen. Personas sind im Bereich der Softwareentwicklung ein probates Mittel, um eine Zielgruppe zu identifizieren, definieren und auszugestalten. ${ }^{298}$

Als archetypische Nutzer von Software beruhen Personas auf Analysen, statistischen Daten, Tests, Beobachtungen und sonstigen vorhandenen Informationen. ${ }^{299}$ Darüber hinaus werden die Profile der skizzierten Nutzergruppen um weitere persönliche Fakten angereichert und repräsentieren die Bedürfnisse, Charakteristika sowie Ziele einzelner potentieller Anwendergruppen. ${ }^{300}$ Sie dienen folglich primär dem Verständnis der Anwenderbedürfnisse.

Aus den Ergebnissen der Anforderungsanalyse lassen sich insgesamt fünf Zielgruppen des Systems ableiten. Die Hauptzielgruppen des Systems sind in diesem Sinne die Nutzer des Philologicums, beispielsweise Studenten der Fakultät für Sprach- und

\footnotetext{
${ }^{295}$ Die vollständigen Baupläne können aufgrund der Rechte der Architekten nicht in die Arbeit übernommen werden.

Pfad: ../Anhang/Baupläne/

${ }^{296}$ Die Kunst am Bau ist in Form von blauen Linien eingezeichnet.

${ }^{297}$ Pfad: ../Anhang/Kunst am Bau/

${ }^{298} \mathrm{Vgl}$. Cooper 2018.

${ }^{299}$ Vgl. Nielsen und Storgaard Hansen 2014, S. $1665 f$.

${ }^{300} \mathrm{Vgl}$. Holt et al. 2010.
} 
Literaturwissenschaft. Des Weiteren die Mitarbeiter der UB, die das zu konzipierende System administrieren. Die Randzielgruppen setzen sich unter anderem aus den Studenten anderer Studienrichtungen der LMU, externen Nutzern und wissenschaftlichen Mitarbeiter der Hochschule zusammen. Für die ersten vier genannten potentiellen Nutzergruppen ist im Folgenden ein Profil in Form von Personas entworfen werden. Auf die Entwicklung einer expliziten Persona für die wissenschaftlichen Mitarbeiter der Hochschule ist verzichtet worden, da sich deren Profil in den anderen entwickelten Personas wiederspiegelt und keine zusätzlichen Anforderungen an das System mit sich bringt.

Die Entwicklung der Personas basiert zum einen, wie bereits einleitend erwähnt auf statistischen Daten in Form von allgemeinen Studien und anonymisierten Daten der Hochschulverwaltung der LMU. ${ }^{301}$ Zum anderen liegen den Personas klar definierte User Stories zu Grunde, die gleichfalls funktionale und nicht funktionale Anforderungen des Systems wiederspiegeln. Darüber hinaus komplettiert die Anforderungsanalyse die entwickelten Personas und ist im Gesamtzusammenhang zu betrachten. Abschließend sei erwähnt, dass der Neubau des Philologicums noch im Bau befindlich ist und die Entwicklung eines Systems zum Angebot von standortbezogenen Informationen gerade angekündigt ist.

\footnotetext{
${ }^{301}$ Vgl. Grohnert 2016 \& vgl. Simon 2018 \& vgl. TERRITORY Embrace 2017 \& vgl. Statista 2017 \& vgl. Statista 2018.
} 


\subsubsection{Persona - Lisa Huber}

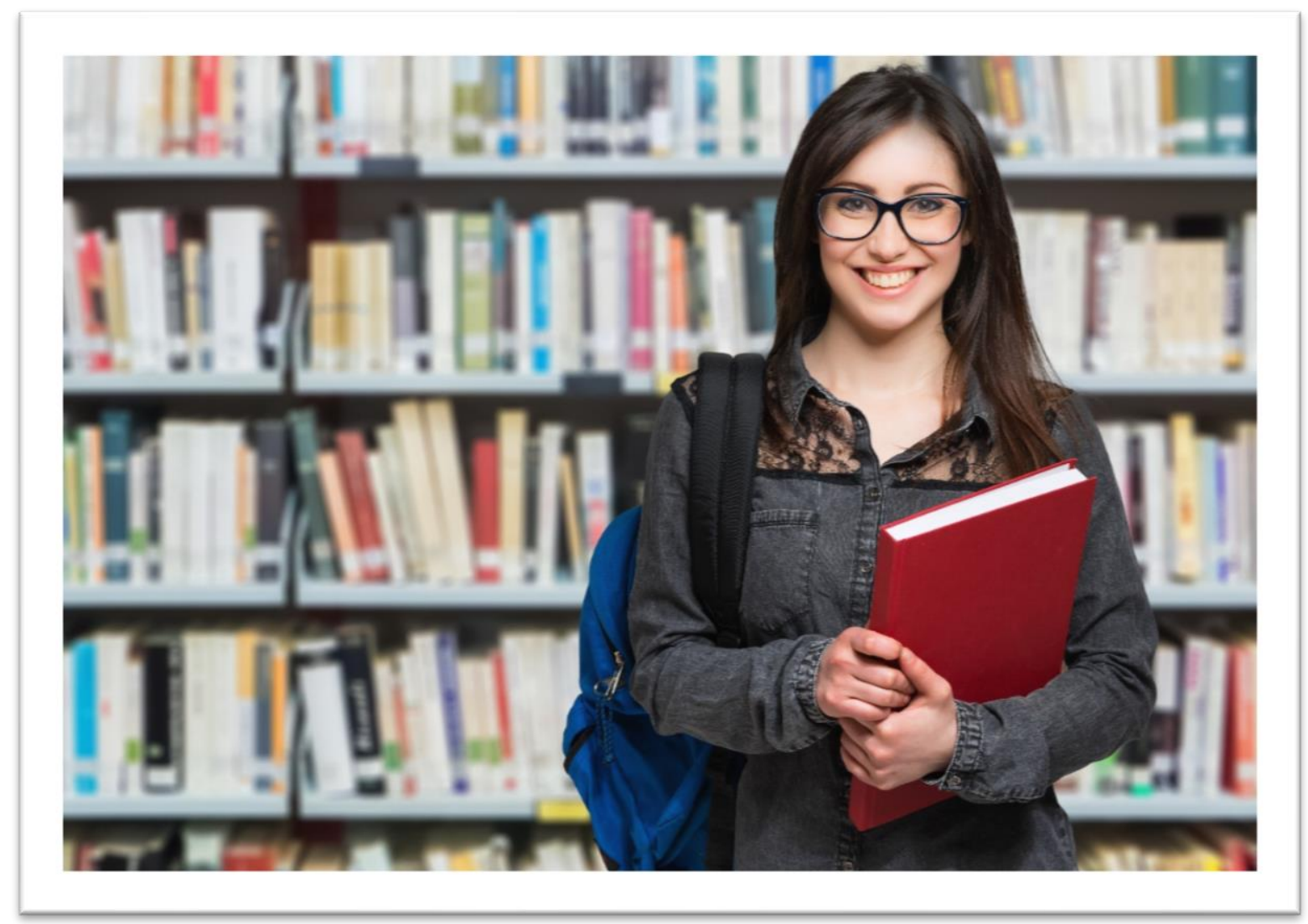

Abbildung 23: Persona Lisa Huber ${ }^{302}$

Lisa Huber ist eine ledige Studentin der Sprach- und Literaturwissenschaft. Sie ist 20 Jahre alt und studiert seit dem Wintersemester 2017 an der LMU München. Vor Ihrem Studienbeginn hat sie das Maria-Theresia-Gymnasium besucht und ihr Abitur mit gutem Erfolg abgelegt. Lisa hat sich für das B.A. Studium „Sprache, Literatur, Kultur“ entschieden, da sie bereits in der gymnasialen Oberstufe Ihr Interesse für deutsche und englische Literatur entdeckt hat.

Mit Ihrer Immatrikulation hat sich Lisa als Nutzerin der UB angemeldet. Neben der aktuellen Literatur im Präsenz- und Magazinbestand, ist für sie vor allem eine ruhige Lern- und Arbeitsatmosphäre im zukünftigen Philologicum wichtig. Da das kooperative Arbeiten in Ihrem Studium fokussiert wird, würde Lisa die Gruppenarbeitsräume des Philologicums gerne für den studentischen Austausch nutzen.

Für Lisa wird der Neubau ein zentraler Lernort sein. Sie möchte deswegen gern über Neuigkeiten, beispielsweise außergewöhnliche Schließzeiten oder verfügbare

${ }^{302}$ Quelle: https://stock.adobe.com/de/images/young-student-in-a-library/103932333 
Seminare fortlaufend informiert werden. Für Lisa ist Ihr Android Smartphone, ein Samsung Galaxy A5 2017, seit dem ersten Tag ein nicht mehr wegzudenkender Begleiter. Sie hat bei einem Gespräch mit einer Mitarbeiterin der UB erfahren, dass im neuen Philologicum interessante Kunst am Bau zu entdecken sein wird. Die Bibliothekarin hat darüber hinaus verraten, dass wohl überwiegend Zitate aus verschiedenster bedeutender Literatur auf großen Glasscheiben angebracht werden. Lisa kann sich gut vorstellen, dass Sie vor einer dieser Glasscheiben steht und die Zitate begutachtet. Ihr kommt dabei die Idee wie genial es wäre, wenn eine App der UB auf ihrem Smartphone zusätzliche Hinweise anzeigen würde. Ihr würde es gefallen, wenn Sie dabei nicht umständlich weitere Eingaben machen müsste, sondern in einer Art Entdeckungsmodus interaktiv interessante Informationen angeboten bekommt.

Darüber hinaus würde sie es praktisch finden, wenn man die Raumbuchungen nicht immer persönlich bei einem Mitarbeiter tätigen müsste, sondern dies bequem aus einer App heraus machen kann. Lisa hat in der Vergangenheit schon viel Zeit darauf verwenden müssen, in ihrer bisherigen Teilbibliothek einen entsprechenden Mitarbeiter für ihr Anliegen zu finden.

Aufgrund Ihrer Verbundenheit zur Literatur ist für sie die Arbeit mit Printpublikationen selbstverständlich. Enzyklopädien und allgemeine Nachschlagewerke nutzt sie jedoch gern elektronisch. Lisa würde es deswegen großartig finden, wenn sie beim Stöbern im Freihandbestand sehen würde, welche der Werke oder weiterführende Datenbanken im elektronischen Portfolio der UB vorhanden sind.

Sie mag gern intuitiv zu bedienende Apps und hat Bedenken, dass die Anwendung zu komplex ist. Des Weiteren sorgt sich Lisa, dass sie nach einer erfolgreichen Raumreservierung vergisst, diese Ihren Kommilitonen mitzuteilen und das die App keine unmittelbare Benachrichtigung weiterer Personen zulässt. 


\subsubsection{Persona - Krista Vogeler}

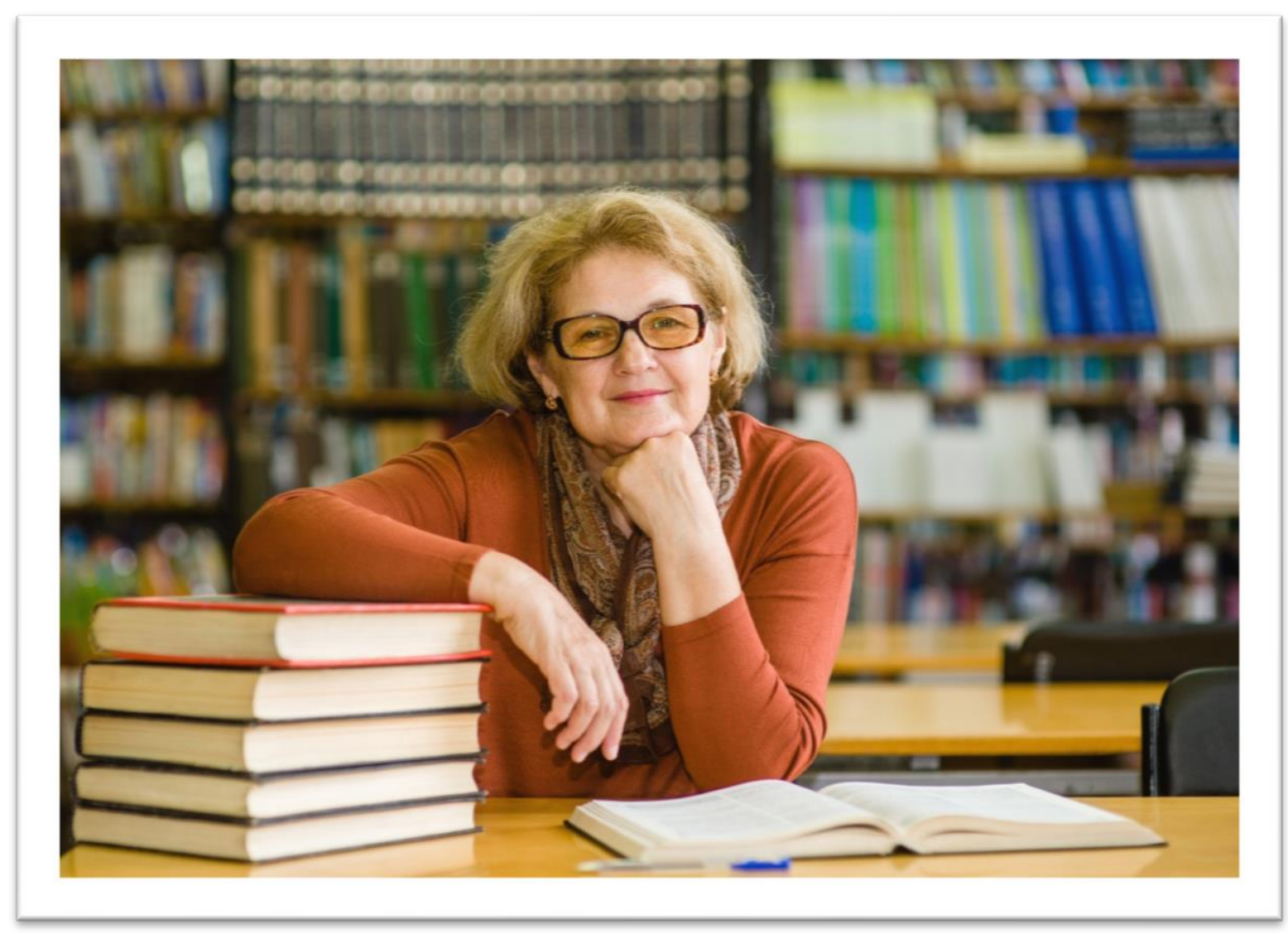

Abbildung 24: Persona Krista Vogeler ${ }^{303}$

Krista Vogeler, eine 57-jährige angestellte Diplom-Bibliothekarin der UB, ist verheiratet und hat 2 Kinder. Insgesamt ist sie schon über 20 Jahre innerhalb der UB in verschiedenen Funktionen tätig gewesen. Angefangen mit einer klassischen Tätigkeit in der Katalogisierung, damals noch im Zettelkatalog, hat Krista im Laufe der Zeit ihre Freude am Umgang mit Menschen und die Begeisterung für die Thekenarbeit, direkt am Nutzer entdeckt. Mit Einzug der einzelnen Fachbibliotheken in den Neubau des Philologicums soll Sie vor Ort Ansprechpartnerin in der Benutzung sein. Ihre berufliche Passion ist es, mit den Studierenden und wissenschaftlichen Mitarbeitern der LMU tagtäglich in Kontakt zu treten, um deren Interessen bestmöglich bedienen zu können. Während Ihrer Laufbahn hat Krista den sicheren Umgang mit dem PC und seinen diversen Anwendungen verinnerlicht. Sie weiß jedoch auch, dass dies nicht immer ein leichtes Unterfangen gewesen ist. Generell hat sie Angst davor, dass die technologische Entwicklung inr den Job kosten könnte.

\footnotetext{
${ }^{303}$ Quelle: https://stock.adobe.com/de/images/happy-senior-woman-in-library/179533011
} 
In einer Dienstbesprechung erfährt Sie von ihrer Sachgebietsleitung, dass die UB eine neue mobile Anwendung für das Philologicum einführen möchte. Die Pläne der Leitung sehen vor, das bisherige CMS als Teil des neuen Systems zu integrieren. Krista hat schon oft mit dieser Software gearbeitet und kennt sich gut darin aus. Jedoch könne das Programm wohl nicht so weit angepasst werden, dass eine vollständige Verwaltung darin möglich sei. Darum soll ein einfach zu bedienendes Tool entwickelt werden, dass die Verknüpfung eines Standortes mit Informationen ermöglicht und so eine Brücke zwischen dem CMS sowie der mobilen Anwendung schlägt. So hat Krista es zumindest verstanden.

Ziel der Besprechung ist es vor allem herauszufinden, wie das CMS angepasst werden muss, um es für das neue System nutzen zu können. Nach Kristas Meinung sollte es vor allem möglich sein, verschiedene Inhalte transportieren zu können. Dies würde ihr ermöglichen, flexibel auf unterschiedliche Gegebenheiten zu reagieren. Darüber hinaus findet sie es wichtig, eine Gültigkeitsdauer für beispielsweise Sofortmeldungen festlegen zu können. Des Weiteren würde sie sich wünschen, dass bereits eingestellte, aber nicht mehr gültige Informationen weiterhin im CMS abrufbar sind. Dies wäre ihrer Meinung nach praktisch, um den Überblick zu behalten.

Krista versteht im Laufe der Besprechung immer besser, warum es ein weiteres Programm als Vermittler zwischen den Inhalten und der Anzeige geben sollte. Sie stellt sich vor, dass es auch für sie, ohne weitere Vorkenntnisse intuitiv zu bedienen ist. Nach Wortmeldungen von Kollegen wirft sie in die Runde, dass es doch reichen sollte, damit eine Verbindung zwischen Standort und Inhalt herstellen zu können und diese wieder zu lösen. Man bräuchte natürlich eine Übersicht über die Standorte schlussfolgert sie in einer lauten Überlegung. „Dafür haben wir doch die Baupläne“ ergänzt ein anderer Kollege.

Jedenfalls ist Krista erleichtert, sich in den letzten Jahren vor Ihrer Rente nicht noch in ein neues, hoch komplexes System einarbeiten zu müssen und freut sich über ein zusätzliches Instrument zur Kommunikation mit den Studierenden. Sie hegt die Hoffnung, dass immer alles reibungslos funktioniert wie gewünscht, denn für sie als technischen Laien ist eine Fehlersuche in der Regel nur mit Unterstützung möglich. 


\subsubsection{Persona - Kevin Lehmann}

Kevin Lehmann ist ein lediger, 25 Jahre alter Masterstudent der Fachrichtung Informatik. Er hat bereits seinen Bachelor an der LMU München absolviert und ist mit dem Umgang der UB vertraut. Aktuell konzentriert sich Kevin auf seine Abschlussarbeit und sucht vor allem eine ruhige Arbeitsatmosphäre. Er wohnt unweit der Fachbibliothek Mathematik und Physik, jedoch bietet diese Zweigstelle der UB keine Gruppenarbeitsräume an und ist zu Stoßzeiten immer sehr gut besucht, sodass er meist nur zufällig einen Platz ergattern kann. Da das Philologicum nicht weit von seiner Wohnung entfernt sein wird, möchte Kevin diese Fachbibliothek zukünftig gern als Ausweichmöglichkeit nutzen.

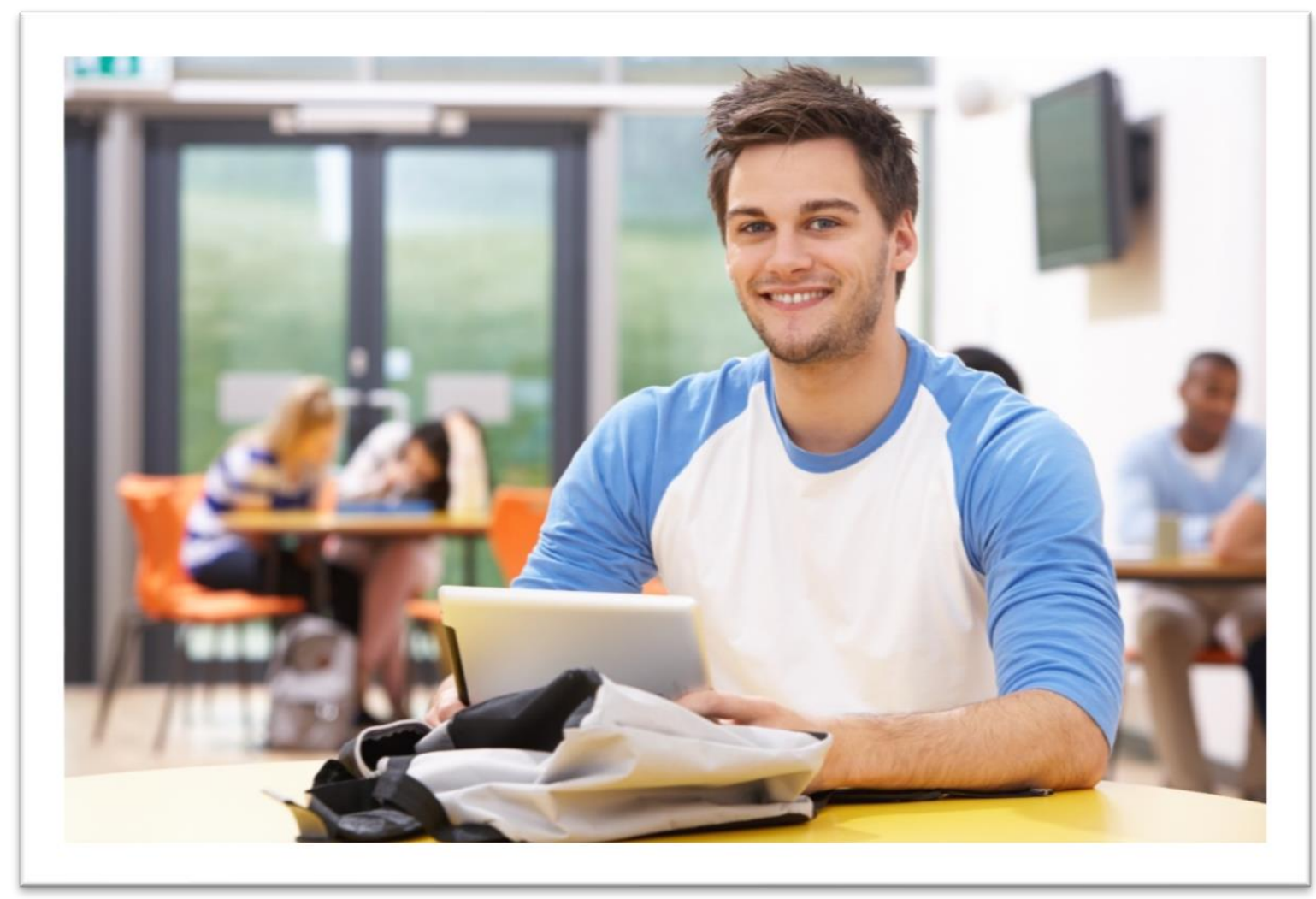

Abbildung 25: Persona Kevin Lehmann ${ }^{304}$

Als technikaffiner Digital Native ist für ihn ein Highendgerät als mobiler Begleiter äuBerst wichtig. Häufig nimmt Kevin sein Studium voll in Beschlag, sodass er nur bei Gelegenheit als wissenschaftliche Hilfskraft an der Universität arbeiten kann. Um Geld zu sparen, hat er sich darum für ein Smartphone abseits der großen Hersteller entschieden. Er wollte im Hinblick auf die Ausstattung indessen keine Abstriche

\footnotetext{
${ }^{304}$ Quelle: https://stock.adobe.com/de/images/male-student-studying-in-classroom-with-digital-tablet/61323371
} 
hinnehmen. In seinem privaten Umfeld erleichtert er sich das Leben mit zahlreichen praktischen Apps. Er hofft, dass auch die UB einen passenden Dienst zur Verfügung stellen wird. Idealerweise informiert dieser ihn über die Verfügbarkeit von Arbeitsplätzen sowie außerplanmäßige Schließzeiten, denn nichts wäre für Kevin ärgerlicher als einen vermeidbaren Weg umsonst anzutreten. Darüber hinaus ist es für ihn hilfreich, sich in einer mobilen Anwendung über allgemeine Informationen, wie Öffnungszeiten oder Telefonnummern für spezielle Ansprechpartner zu erkundigen.

Kevin ist es in anderen Fachbibliotheken der LMU schon oft passiert, dass Gruppenarbeitsräume auf der Website zwar als reserviert angezeigt worden sind, tatsächlich dann aber niemand da war und diese in Anspruch genommen hat. Er wäre begeistert, wenn man einfach direkt am Gruppenarbeitsraum vor Ort prüfen könnte, ob dieser belegt ist oder nicht. Damit er ihn kurzfristig nutzen kann.

In der Vergangenheit hat er sich oft darüber geärgert, dass er die Bedienung von Druckern erst durch umständliches Ausprobieren am Gerät herausfinden musste. Gerade solche Spezialitäten wie Duplexdruck oder eine Vergrößerung von Dokumenten hat inn bisher viel Zeit und Geld gekostet. Kevin fände es innovativ, wenn er eine Anleitung für solche speziellen Fälle mit seinem Smartphone abrufen könnte.

Kevin mag es gar nicht in der heutigen Zeit zusätzliche Eingaben in sein Smartphone tippen zu müssen. Für ihn sollte idealerweise alles einfach automatisch funktionieren.

\subsubsection{Persona - Louis Momodou}

Louis Momodou ist ein Literaturwissenschaftler aus Südafrika. Er ist 33 Jahre alt und nicht verheiratet. Vor ein paar Jahren hat er seine Leidenschaft für historische Literatur deutscher Dichter entdeckt. Passenderweise hat sein Arbeitgeber, eine Universität in seinem Heimatland, ihm ein Projekt in diesem Themenbereich übertragen. Mit einer einjährigen Forschungsreise nach Deutschland möchte er seine Grundkenntnisse der deutschen Sprache weiter ausbauen und Primärquellen sowie passende Sekundärliteratur zu seinem Projekt sammeln. Über einen ortsansässigen, befreundeten Literaturwissenschaftler hat Louis vom Neubau des Philologicums und seinem Bestand erfahren. Vor allem interessiert inn der Präsenzbestand der Fachbibliothek sowie die Kunst am Bau, über die er gerne mehr Hintergrundinformationen in Erfahrung bringen 
würde. Er hofft auch als nicht Universitätsangehöriger die Bibliothek und ihre Dienstleistungen nutzen zu können.

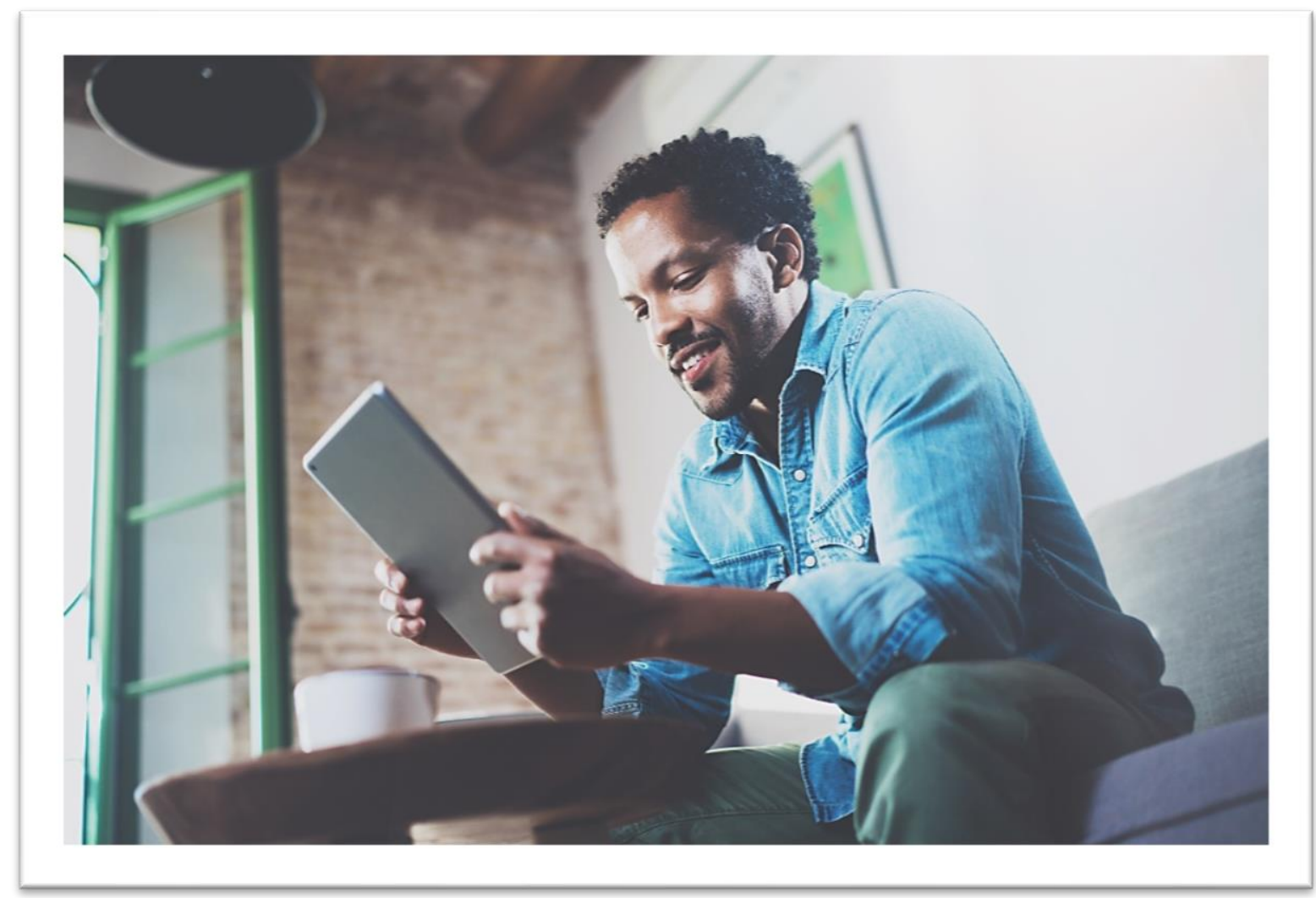

Abbildung 26: Persona Louis Momodou ${ }^{305}$

Sein Bekannter hat inm erzählt, dass die UB eine mobile Anwendung entwickelt, die Informationen rund um das Philologicum anbieten wird. Louis findet das Vorhaben klasse, da er sich vor kurzem ein neues Smartphone in der Einstiegsklasse zugelegt und sein altes Handy ausrangiert hat. Seitdem ist er auf der Suche nach informativen sowie hilfreichen Apps.

Louis kennt sich in München nicht aus und nutzt momentan Google Maps, um sich von A nach B zu navigieren. Er hofft, dass er neben der allgemeinen Angabe des Standortes gleichfalls eine Verknüpfung zu Google Maps vorfindet, um auf dem kürzesten Weg zum Neubau zu gelangen.

Aus den Gesprächen mit seinem Freund hat er erfahren, dass das Philologicum sehr übersichtlich gestaltet sein soll. Dennoch hat Louis es sich zur Angewohnheit gemacht, sich gern im Vorhinein, über die örtlichen Gegebenheiten zu informieren. Er

\footnotetext{
${ }^{305}$ Quelle: https://stock.adobe.com/de/images/bearded-smiling-african-man-using-tablet-for-videoconversation-while-relaxing-on-sofa-in-modern-office-concept-of-young-business-people-workingat-home-blurred-background-selective-focus/135174678
} 
wäre glücklich, wenn er auf einem Lageplan nachschauen könnte wo sich was im Neubau befindet, denn er irrt nicht gern in ungewohnten Lokalitäten planlos umher. Wie in seiner Heimatregion üblich spricht Louis fließend Englisch. Er wünscht sich ebenfalls ein elektronisches Informationsangebot in dieser Sprache, da er Angst hat, dass er auf Deutsch nicht alles verstehen würde. Darüber hinaus hat Louis die Befürchtung, dass die App auf seinem Android-Einstiegsgerät nicht lauffähig sein könnte. 


\section{Evaluation und Testszenarien}

\subsection{Evaluation}

Prinzipiell könnte mit jeder der vorgestellten Technologien in Kapitel 5 eine Konzeption und Realisierung eines funktionstüchtigen IPS für die Auftraggeberin erstellt werden. Aufgrund der erarbeiteten spezifischen Vor- und Nachteile sowie den gestellten Anforderungen und zukünftigen Anwendungsszenarien soll in diesem Kapitel eine kleine Schnittmenge optimal passender Technologien und Techniken selektiert werden.

Zur Wahrung einer kompakten und nachvollziehbaren Darstellung wird im Folgenden eine kaskadierende Evaluation vorgenommen. Jede Stufe der Bewertung beinhaltet ein oder mehrere KO-Kriterien, die eine Filterung der übrigbleibenden möglichen Technologien sicherstellen. Diese KO-Kriterien leiten sich aus der Anforderungsanalyse $a b$ und stellen sicher, dass den Bedürfnissen der Auftraggeberin vollumfänglich, vor allem im Hinblick auf die Priorisierung, Rechnung getragen wird.

Nach Erreichen der letzten Selektionsstufe werden die verbleibenden Technologien einer praktischen Untersuchung unterzogen, um beispielsweise weitere, sekundäre Kriterien zu bewertet. Ziel dieser abschließenden Analyse soll es sein, wichtige zu beachtende Faktoren, bezüglich der Konzeption und prototypischen Umsetzung zu identifizieren.

\subsubsection{Evaluationsstufe 1 - Sensorik und Zukunftsfähigkeit}

In der ersten Evaluationsstufe werden die vorgestellten Technologien anhand der Kriterien "Sensorik“ und „Zukunftsfähigkeit" bewertet. Diese leiten sich aus den Anforderungen FE 0010 und FE 0020 der vorangegangenen Anforderungsanalyse ab. Die Rahmenbedingungen der Auftraggeberin sehen vor, dass das Frontend als Softwareanwendung auf Basis eines mobilen Endgerätes entwickelt wird. Diese Anforderung wird durch die theoretischen Erkenntnisse aus Kapitel 3 und 4 untermauert. Folglich ist das erste KO-Kriterium, dass zu einer Disqualifizierung einer Technologie führt, die Verbreitung der Sensoren in Smartphones und dessen Zukunftsfähigkeit. Sollte jeweils bei beiden Merkmalen das Attribut "gering" vergeben werden, dann wird die Technologie herausgefiltert. 


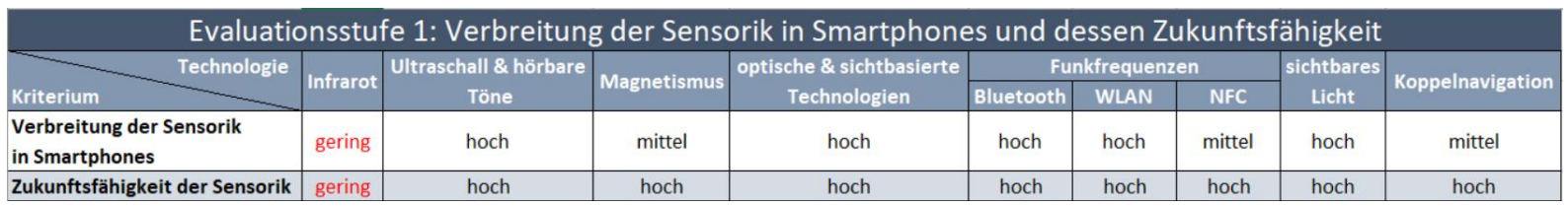

Tabelle 7: Evaluationsstufe 1

Die Attribuierung „hoch“ ist vergeben worden, wenn nahezu alle Endgeräte diese Hardwarekomponente aufweisen. Entsprechend hoch ist folglich auch deren $\mathrm{Zu}$ kunftsfähigkeit eingeschätzt worden. Sollte eine Vielzahl von Endgeräten, mindestens jedoch jedes Dritte, eine Hardwarekomponente enthalten, dann ist die Bewertung in der Kategorie „mittel“ erfolgt. Bei den analysierten Sensoren für Magnetismus, NFC und Koppelnavigation ist dennoch ein hohes Potential für die Zukunftsfähigkeit evaluiert worden, da zum aktuellen Zeitpunkt keine Anzeichen, für ein Verschwinden der Bauteile aus dem Smartphonemarkt vorhanden sind.

Lediglich die Technologie Infrarot, die sich der entsprechenden Infrarotschnittstelle bedient hat eine sehr geringe Verbreitung im derzeitigen Smartphonemarkt. Aufgrund der Marktdurchdringung von gerade einmal 7\% wird dieser Komponenten keine Zukunftsfähigkeit vorhergesagt. Aufgrund der Erfüllung des zuvor definierten KO-Kriteriums, ist Infrarot die erste ausscheidende Technologie.

\subsubsection{Evaluationsstufe 2 - zusätzliche technische Infrastruktur und Kosten}

In der Evaluationsstufe 2 sind die verbleibenden Technologien auf die Anforderungen IP 0080 und IP 0100 hin, keine zusätzliche technische Infrastruktur zu benötigen, eruiert worden. Zur Disqualifizierung einer Technologie führt, wenn diese die besagte Infrastruktur benötigt und deren Anschaffungskosten hoch sind. Die ausschließende Attribuierung ist bewusst in dieser Kombination gewählt worden, um keine potentiell vielversprechende Technologie zu früh herauszufiltern. Jedoch kann eine entsprechende Ausprägung bereits Indikator für dessen zukünftige Eignung sein.

\begin{tabular}{|c|c|c|c|c|c|c|c|c|}
\hline \multicolumn{9}{|c|}{ Evaluationsstufe 2: zusätzliche technische Infrastruktur und Kosten } \\
\hline \multirow[b]{2}{*}{ Kriterium } & \multirow{2}{*}{$\begin{array}{l}\text { Ultraschall \& hörbare } \\
\text { Töne }\end{array}$} & \multirow{2}{*}{ Magnetismus } & \multirow{2}{*}{$\begin{array}{c}\text { optische } \& \text { sichtbasierte } \\
\text { Technologien }\end{array}$} & \multicolumn{3}{|c|}{ Funkfrequenzen } & \multirow{2}{*}{\begin{tabular}{|c|} 
sichtbares \\
Licht
\end{tabular}} & \multirow{2}{*}{ Koppelnavigation } \\
\hline & & & & \begin{tabular}{|l|} 
Bluetooth \\
\end{tabular} & WLAN & NFC & & \\
\hline $\begin{array}{l}\text { zusätzliche technische } \\
\text { Infrastruktur }\end{array}$ & ja & nein & nein & ja & nein & ja & ja & nein \\
\hline $\begin{array}{l}\text { Kosten der zusätzlichen } \\
\text { technischen Infrastruktur }\end{array}$ & hoch & keine & keine & mittel & keine & gering & hoch & keine \\
\hline
\end{tabular}

Tabelle 8: Evaluationsstufe 2 
Die Hälfte der verfügbaren Technologien sind auf die Installation zusätzlicher technischer Infrastruktur angewiesen, jedoch variieren die entstehenden Anschaffungskosten. NFC ist mit geringen initialen Kosten evaluiert worden, da sich die Preise für NFCTags im Bereich von Cents bewegen. ${ }^{306}$ Die Kosten für BLE-Beacons sind als deutlich teurer im Vergleich zu NFC-Tags eingeschätzt worden, jedoch günstiger, als die benötigten Komponenten für Ultraschall und sichtbares Licht. Demnach sind die Kosten mit der Ausprägung „mittel“ bewertet worden.

Ultraschall und hörbare Töne sowie sichtbares Licht benötigen beide Infrastruktur, die mit hohen Kosten veranschlagt werden. Wie in den einleitenden theoretischen Kapiteln beschrieben, benötigen diese Technologien zentrale Steuereinheiten und weitere Komponenten, die aufgrund ihrer speziellen Anforderungen in einer preislich anderen Klasse als BT und NFC spielen. Aufgrund der Erfüllung der KO-Kriterien findet an dieser Stelle ein Ausscheiden statt.

\subsubsection{Evaluationsstufe 3 - Initialisierungs- und Wartungsaufwand}

Die dritte und vorletzte Evaluationsstufe beschreibt die Kriterien Initialisierungs- und Wartungsaufwand. Basis für diese KO-Kriterien sind die Anforderungen IP 0100 und IP 0110. Es ist anzumerken, dass der Initialisierungsaufwand nicht den Faktor Kosten beinhaltet, da dieser bereits in der Evaluationsstufe 2 eruiert worden ist. Vielmehr meint der Initialisierungsaufwand den Faktor Personalaufwand, der betrieben werde muss, um eine Technologie zur Standortbestimmung einsatzbereit zu machen. Der Wartungsaufwand hingegen inkludiert sowohl Materialkosten, als auch benötigte personelle Kapazitäten, um die Technologie zu unterhalten sowie einen funktionstüchtigen Zustand zu gewährleisten. Zum Ausscheiden einer Technologie führt eine Kombination aus hohem Initialisierungs- und Wartungsaufwand.

In den ersten beiden Evaluationsstufen hat im Hinblick auf die Technologie WLAN keine Berücksichtigung bezüglich der Kombination mit einzelnen Algorithmen stattgefunden, da die Kriterien eine allgemeine Bedeutung auf gesamter Technologieebene hatten. In dieser Stufe spielen jedoch die einzelnen Algorithmen eine bedeutungstragende Rolle, da mit ihnen spezifische Ausprägungen, der zu bewertenden

\footnotetext{
${ }^{306}$ Vgl. NFC21 GmbH.
} 
Kriterien auftreten. Zur besseren Analyse ist eine granulare Feinjustierung in Form der Ergänzung aller Kombinationsmöglichkeiten vorgenommen worden.

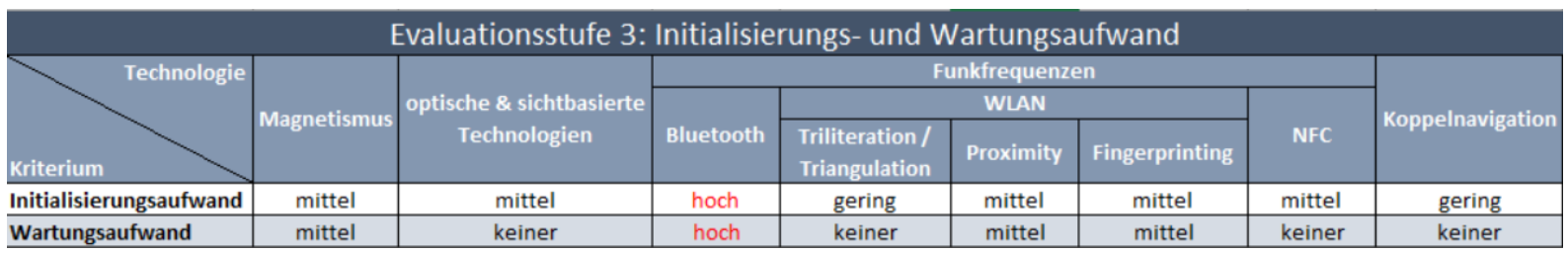

Tabelle 9: Evaluationsstufe 3

Beide Aufwände werden Bezug nehmend auf die Technologie Magnetismus als mittel eingeschätzt. Sie definieren sich vor allem in der Tatsache, dass sowohl initial, als auch im Rahmen von Wartungsintervallen eindeutige Fingerprints gesammelt werden müssen, um dauerhaft eine funktionstüchtige Standortbestimmung zu gewährleisten. Zum Einmessen der Signaturen benötigt es vor allem Personal, dass dieses an einer Vielzahl von Punkten durchführt, denn je mehr Werte für den späteren Abgleich vorhanden sind, desto genauer wird der Algorithmus arbeiten können.

Selbiges gilt für WLAN in Kombination mit Fingerprinting. Auch bei einer Verwendung von Proximity muss zunächst durch einen Mitarbeiter an verschiedenen Standorten ein Referenz-RSSI eingelesen werden. Darüber hinaus sollte dieser in regelmäßigen Intervallen nach justiert werden. Die verbleibende Standortbestimmung basierend auf WLAN, mit den Algorithmen Trilateration und Triangulation haben nur einen geringen initialen Aufwand und beschränken sich auf das Testen der korrekten Funktionsweise. Ein Wartungsaufwand ist praktisch nicht vorhanden, da dieser Algorithmus autark arbeitet und nicht auf vorher eingemessenen Werten beruht.

Bei der Koppelnavigation zeichnet sich ein ähnliches Bild, da bei fehlerfreier Arbeitsweise der Technologie keine externe Wartung notwendig ist, sondern die Positionsbestimmung vollständig mit Werten, die das Smartphone selbstständig liefert, funktioniert. Sicher sollte die vorschriftsmäßige Standortbestimmung im Vorfeld erprobt werden. Aus diesem Grund lässt sich ein minimaler Initialisierungsaufwand verzeichnen.

NFC und optische sowie sichtbasierte Technologien zeichnen sich durch einen kongruenten Initialisierungsaufwand aus. Beide bieten auf unterschiedliche Art und Weise ein Trägermedium zur persistenten Sicherung von Informationen, beispielsweise einem Standort in Form einer codierten ID an. Es entsteht vor allem ein personeller Aufwand bei dem Vorgang der Codierung. Sobald diese abgeschossen und eine 
Platzierung an dem jeweiligen Standort erfolgt ist, muss keinerlei regelmäßiger Wartungsaufwand betrieben werden, damit eine Standortbestimmung möglich ist.

BT scheidet in dieser Evaluationsstufe aus, da beide Aufwände als sehr hoch bewertet werden. Zum einen ist ein hoher Personalaufwand erforderlich, um die Beacons an Ort und Stelle zu positionieren, und zum anderen ist der Wartungsaufwand, gerade im Vergleich zu den anderen Technologien, um ein Vielfaches höher. Gerade in einem Szenario, wo eine Vielzahl an Beacons verwendet wird steigt der Wartungsaufwand exorbitant an. Die Kosten sowie der Bedarf an Personal, beispielsweise bei einem Batteriewechsel, übersteigen die verglichenen Technologien bei weitem und sind Grund für die Disqualifikation.

\subsubsection{Evaluationsstufe 4 - Anfälligkeit für Störfaktoren und Abhängigkeit von ein- heitlichen Messwerten}

Die vierte und letzte vergleichende Bewertungsstufe wertet die Anfälligkeit für »Störfaktoren« und die Abhängigkeit von einheitlichen Messwerten zwischen verschiedenen Endgeräten aus. Diese beiden Kriterien leiten sich aus den Anforderungen IP 0030, IP 0040 und IP 0120 sowie den aufgezeigten Abhängigkeiten in den Kapiteln 5.3.2 und 5.4 ab. Die Anfälligkeit für »Störfaktoren« ist im Wesentlichen auf Algorithmus- und Technologieebene beschrieben und genannt worden. Die Abhängigkeit von einheitlichen Messwerten zur korrekten Positionsbestimmung basiert vor allem auf der logischen Schlussfolgerung für die verbleibenden Technologien. Zum Ausschluss einer Technologie oder einer Kombination dieser mit einem Positionierungsalgorithmus führt die Bewertung mit dem Attribut „hoch“ in einer der beiden Kriterien.

\begin{tabular}{|c|c|c|c|c|c|c|c|}
\hline Technologie & \multirow{3}{*}{ Magnetismus } & \multirow{3}{*}{$\begin{array}{l}\text { optische \& sichtbasierte } \\
\text { Technologien }\end{array}$} & \multicolumn{4}{|c|}{ Funkfrequenzen } & \multirow{3}{*}{ Koppelnavigation } \\
\hline \multirow{3}{*}{$\begin{array}{l}\text { Evaluationskriteriu } \\
\text { Anfälligkeit für Störfaktoren }\end{array}$} & & & & WLAN & & & \\
\hline & & & $\begin{array}{l}\text { Triliteration / } \\
\text { Triangulation }\end{array}$ & Proximity & Fingerprinting & NFC & \\
\hline & hoch & gering & hoch & hoch & hoch & gering & hoch \\
\hline $\begin{array}{l}\text { Abhängigkeit von einheitlichen } \\
\text { Messwerten auf unterschiedlichen } \\
\text { Endgeräten }\end{array}$ & hoch & gering & hoch & hoch & hoch & gering & gering \\
\hline
\end{tabular}

Tabelle 10: Evaluationsstufe 4

Gerade im Hinblick auf Technologien, die auf dem Vergleichen von zuvor erfassten Messwerten beruhen, ist die Abhängigkeit von einheitlichen Messwerten sehr hoch. Zur besseren Verdeutlichung, wie verschieden die ausgelesenen Messwerte bei 
unterschiedlichen Endgeräten sind, ist für Magnetismus und WLAN jeweils eine Anwendung programmiert worden, die diese erfassen, visualisieren und speichern. ${ }^{307}$

\begin{tabular}{|l|r|r|r|r|}
\hline \multirow{2}{*}{ Smartphone } & \multicolumn{4}{|c|}{ Durchschnitt } \\
\cline { 2 - 5 } & $\mathrm{X}$ & \multicolumn{1}{c|}{$\mathrm{Y}$} & \multicolumn{1}{c|}{$\mathrm{Z}$} & Magnitude \\
\hline OnePlus 6 & $-7,012088095$ & $-14,23957245$ & $-12,11869313$ & 19,97401538 \\
\hline Samsung Galaxy A5 & 15,13979975 & 1,77119998 & $-20,86379982$ & 25,84369265 \\
\hline Samsung Galaxy A3 & 16,13881993 & $-0,391249771$ & $-20,31060059$ & 26,355439 \\
\hline \hline $\begin{array}{l}\text { maximale Abweichung } \\
\text { der Messwerte }\end{array}$ & 23,15090802 & 16,01077243 & 8,74510669 & 6,381423617 \\
\hline
\end{tabular}

Tabelle 11: Auszug der Messwertanalyse mittels der App „MagneticFingerprint“

In einem Test sind Mithilfe der App „MagneticFingerprint“ einhundert Signaturen des magnetischen Umfeldes an einem festen Standort mit drei unterschiedlichen Endgeräten gesammelt worden. ${ }^{308}$ Es ist zu beachten, dass die Geräte im Vorhinein kalibriert worden sind. Während des Tests sind diese an einer festen Position im Raum fixiert und nicht bewegt worden, bis die Erfassung der Fingerprints vollständig abgeschlossen gewesen ist. Diese idealen Bedingungen würden in einem produktiven Einsatzszenario wohl kaum vorliegen. Tabelle 11 verdeutlicht, dass selbst bei einer kleinen Basis von lediglich drei Endgeräten bereits eine große Varianz der erfassten Sensorwerte vorliegt und somit eine Attribuierung von „hoch“ vergeben worden ist.

\begin{tabular}{|c|c|c|c|}
\hline $\begin{array}{l}\text { OnePlus } 6 \\
\text { Fingerprint } 1\end{array}$ & Samsung Galaxy A3 & Samsung Galaxy A5 & $\begin{array}{l}\text { ZTE Blade L5 Plus } \\
\text { Fingerprint } 1\end{array}$ \\
\hline Model: ONEPLUS A6003 & Model: SM-A310F & Model: SM-A520F & Model: ZTE Blade L5 Plus \\
\hline Time: 2018-09-29 13:54:57 & Time: 2018-09-29 13:54:50 & Time: 2018-09-29 13:54:55 & Time: 2018-09-29 13:54:55 \\
\hline SSID: FritzBox YK | RSSI: -66 & SSID: FritzBox YK | RSSI: -71 & SSID: DIRECT-veM2020 Series | RSSI: -78 & SSID: FritzBox YK | RSSI: -70 \\
\hline SSID: DeathStar_2.4GHz | RSSI: -72 & SSID: DeathStar_2.4GHz | RSSI: -91 & SSID: FritzBox YK | RSSI: -79 & SSID: DIRECT-q3-FireTV_2562 | RSSI: -62 \\
\hline SSID: WLAN-405361 | RSSI: -75 & SSID: DIRECT-veM2020 Series | RSSI: -82 & SSID: DeathStar_2.4GHz | RSSI: -80 & SSID: | RSSI: -62 \\
\hline SSID: DIRECT-veM2020 Series | RSSI: -77 & SSID: WLAN-405361 | RSSI: -83 & & SSID: WLAN-405361 | RSSI: -82 \\
\hline SSID: FRITZ!Box 7560 GK | RSSI: -83 & SSID: | RSSI: -67 & & SSID: DIRECT-veM2020 Series | RSSI: -73 \\
\hline \multirow[t]{2}{*}{ SSID: FRITZ!Box 7490 | RSSI: -86} & SSID: FRITZ!Box 3272 | RSSI: -86 & & SSID: DeathStar_2.4GHz | RSSI: -81 \\
\hline & & & SSID: FRITZ!Box 3272 | RSSI: -90 \\
\hline \multicolumn{4}{|l|}{ Legende } \\
\hline \multicolumn{4}{|c|}{ Fett = AP's kommen in allen Fingerprints des Endgerätes vor } \\
\hline Rot $=$ AP's kommen bei allen Endgeräten & des Fingerprints vor & & \\
\hline
\end{tabular}

Tabelle 12: Auszug der Messwertanalyse mittels der App „WLANScanner"

Zur Evaluation der Technologie WLAN ist die App „WLANScanner" programmiert worden, um insgesamt zehn Fingerprints von vier Endgeräten zu erfassen. Alle

${ }^{307}$ Der Quellcode für die Apps „MagneticFingerprinting“ und „WLANScanner“ können der CD entnommen werden.

MagneticFingerprinting: Pfad: ../Anhang/App/MagneticFingerprinting/

WLANScanner: Pfad: .../Anhang/App/WLANScanner/

${ }^{308}$ Die vollständige Analyse, Testbedingungen, Anmerkungen und die zugrunde liegenden Daten sind dem Anhang zu entnehmen.

Analyse: Pfad: ../Anhang/Evaluation/Test Magnetismus.xlsx

Rohdaten: Pfad: ../Anhang/Evaluation/Rohdaten/Magnetismus 
Smartphones sind an der gleichen festen Position im Raum nebeneinander fixiert worden. Während der Ausführung der Scans sind die Geräte an dieser Position verblieben und zeitgleich gestartet worden. ${ }^{309}$

Interessant ist die Beobachtung, dass selbst wenn ein AP bei allen Endgeräten innerhalb eines Fingerprints vorkommt, die RSSI-Level fast durchgehend voneinander abweichen. Auch bei dem durchgehenden Vorkommen eines AP's in allen zehn Signaturen, weichen die RSSI-Level häufig voneinander ab und sind selten konstant. Tabelle 12 visualisiert, die Varianz der Messwerte und verdeutlicht deren Uneinheitlichkeit. Der Ausgleich von unterschiedlichen Referenzwerten und deren Zuordnung zu einem Standort im Raum müsste folglich durch einen aufwendigen Algorithmus kompensiert werden.

Negativ auf WLAN wird sich darüber hinaus das mit Android 9 eingeführte „Throttling“ auswirken. Innerhalb einer Zeit von zwei Minuten darf ein Smartphone zukünftig nur noch eine Anzahl von vier WLAN-Scans durchführen. ${ }^{310}$ Weitere Scans werden durch das OS an dieser Stelle dann bis zum Ablauf der Wartezeit unterbunden. Dieses Verhalten ist bei dem Testgerät OnePlus6, dass bereits mit Android 9 ausgestattet ist, beobachtbar, da ab dem vierten Scan keine sich verändernden Messwerte mehr ausgelesen werden.

Optische und sichtbasierte Technologien, NFC und Koppelnavigation zeichnen sich durch eine geringe Abhängigkeit zu einheitlichen Messwerten aus. Im Rahmen dieser Evaluationsstufe verbleiben jedoch, aufgrund der Anfälligkeit für »Störfaktoren« nur die beiden erst genannten Technologien, da diese jeweils in beiden Kriterien ohne eine KO-Attribution bewertet worden sind. Die Koppelnavigation scheidet vor allem aufgrund des „Sensor-Drifts“, beschrieben in Kapitel 5.4.7 aus.

Im Folgenden soll deswegen mit den positiv evaluierten Technologien in praktischen Tests herausgefunden werden, ob sich diese zur Umsetzung der Anforderungen eignen. Sollte dies nicht der Fall sein, so können alternativ Technologien der vierten Evaluationsstufe zur praktischen Erprobung herangezogen werden.

\footnotetext{
${ }^{309}$ Die vollständige Analyse, Testbedingungen, Anmerkungen und die zugrunde liegenden Daten sind dem Anhang zu entnehmen.

Analyse: Pfad: ../Anhang/Evaluation/Test WLAN.xIsx

Rohdaten: Pfad: ../Anhang/Evaluation/Rohdaten/WLAN

${ }^{310} \mathrm{Vgl}$. Google LLC 2018k.
} 


\subsection{Evaluation ausgewählter Technologien mittels praktischer Testszenarien}

Aus den funktionalen Anforderungen der Analyse in Kapitel 6 zeichnet sich ab, dass der LBS der Auftraggeberin ebenfalls, wie grundsätzlich in Kapitel 3 beschrieben, in einen Push- sowie Pull-Dienst unterschieden werden könnte. Zunächst ist die Umsetzung der Anforderung IP 0020 ein klassischer Pull-Dienst, bei dem sich der Nutzer an einem POI zusätzliche Informationen beschaffen möchte. Dieser Service kann mithilfe von Radio Frequency, genauer gesagt NFC und einer Technologie aus der Kategorie Optical and Vision, beispielsweise QR-Codes, exemplarisch umgesetzt werden.

Die Anforderung IP 0010 (Positionsbestimmung „Kunst am Bau) in Kombination mit einer geringen Genauigkeit (IP 0030) und mittleren Reichweite (IP 0050) implizieren auf den ersten Blick eine Umsetzung mittels eines Push-Dienstes. Die sich dafür anbietenden Technologien, wie BT, WLAN oder Magnetismus, sind jedoch im Rahmen der Evaluation in verschiedenen Stufen ausgeschieden. Bei eingehender Betrachtung fällt auf, dass sich diese Standortbestimmung in gleicher Weise mittels eines PullDienstes umsetzen lässt.

Bei der Kunst am Bau handelt es sich immer um Schrift auf Glasscheiben. Eine Möglichkeit der Palette aus den optischen und sichtbasierten Technologien ist die Texterkennung, bei der eine automatisierte optische Zeichenerkennung durchgeführt wird. Begünstigt wird diese Methode durch die Notwendigkeit des Bestehens einer Sichtlinie zur Rezeption der visuellen Kunst durch den Nutzer. Damit transformiert sich die Kunst am Bau gleichfalls zu einem POI, an dem sich der Nutzer weiterführende Informationen anzeigen lassen kann.

Um eine optimale Synthese aus Anforderungen sowie Vor- und Nachteilen einzelner Technologien zur Standortbestimmung herbeizuführen, sollen ausgewählte Testszenarien durchgeführt werden. Ziel der Tests soll die Schaffung einer fundierten Grundlage für Konzeption und prototypischen Umsetzung sein.

Alle Testszenarien werden auf insgesamt vier Smartphones auf Basis von Android durchgeführt, wie in der optionalen Anforderung FE 0080 (Prototyp auf Basis von Android) analysiert. Jedes der Geräte repräsentiert im Hinblick auf Markteinführung und Preis eine unterschiedliche Kategorie, sodass vom Highendgerät bis zur günstigen Einstiegsklasse jeder Gerätetyp vertreten ist. Von Vorteil ist, dass alle Geräte unterschiedliche Hardwareeigenschaften aufweisen und so eine detaillierte Evaluation der 
Technologien erlauben. Die technischen Datenblätter der Smartphones befinden sich im Anhang. ${ }^{311}$

\subsubsection{QR-Codes}

Primär soll bei dieser Untersuchung die Frage geklärt werden, bis zu welchen Grad bzw. bei welchen Abhängigkeiten die Information richtig erkannt wird. Ziel ist es den besten Kompromiss zwischen der höchsten Informationsdichte, bei kleinster QRCode Größe mit bestmöglicher Erkennungsrate zu finden. Die zukünftigen Markierungen sollen gemäß den Anforderungen visuell so unauffällig wie möglich in den Standort integriert werden. Die Informationsdichte ist mit der Codierung verschieden langer IDs bzw. einer URL getestet worden. Zum einen ist eine vierstellige und zum anderen eine zehnstellige ID codiert worden. Bei der URL handelt es sich um eine Adresse zur Reservierung eines Raumes in dem webbasierten Dienst der LMU. Diese besteht insgesamt aus 47 Zeichen.

Die QR-Codes sind mit einem kostenfreien Generator $^{312}$ erstellt worden. Mit $2000 \mathrm{Pi}$ xeln ist für jede Codierung die höchste Auflösung gewählt worden. Neben der Integration des Logos der LMU ist der QR-Code darüber hinaus durch andere optische Veränderungen individualisiert worden, um seine Funktionalität bereits mit modifizierten Bedingungen aussagekräftig untersuchen zu können.

Um der initial zu untersuchenden Fragestellung gerecht zu werden, sind die QRCodes jeweils in unterschiedlichen Größen getestet worden. Konkret sind die Abmaße $3 \mathrm{~cm}^{2}, 5 \mathrm{~cm}^{2}, 8 \mathrm{~cm}^{2}$ und $10 \mathrm{~cm}^{2}$ verwendet worden. Des Weiteren sind die Markierungen nicht durch Farben individualisiert worden, da dies den Ergebnissen der Anforderungsanalyse widersprechen würde. Der somit verwendete maximale Schwarz-WeißKontrast begünstigt dahingehend die Erkennungsrate.

Gemäß den funktionalen Anforderungen wird diese Technologie tendenziell auf kurzen Distanzen eingesetzt werden. Beispielsweise beträgt die Breite eines Korridors, von dem die Einzel- und Gruppenarbeitsräume abzweigen, gemäß den Bauplänen ca. $1,5 \mathrm{~m}$. Aus diesem Grund sind die Entfernungen $15 \mathrm{~cm}, 30 \mathrm{~cm}, 45 \mathrm{~cm}, 60 \mathrm{~cm}$ und 75

\footnotetext{
${ }^{311}$ Pfad: ../Anhang/Evaluation/Datenblätter.xlsx

${ }^{312}$ Vgl. Sroke 2018.
} 
cm gewählt worden. Abschließend sind jeweils ein horizontaler und vertikaler Neigungswinkel zwischen Smartphone und QR-Code in Betracht gezogen worden, um möglichst realitätsnah eine Aussage zur Erkennungsrate aus unterschiedlichen Blickwinkeln treffen zu können.

Die Messungen sind auf allen Smartphones mit einem kostenfreien QR Code Reader ${ }^{313}$ durchgeführt worden. Insgesamt sind im Rahmen der Tests mit allen Geräten 720 Messwerte erhoben worden. Als korrekt erkannt ist eine Messung gewertet worden, wenn die Daten fehlerlos decodiert worden sind und der Vorgang nicht länger als eine Sekunde gedauert hat. Als nicht korrekt ist ein Ergebnis festgehalten worden, bei dem entweder der QR-Code gar nicht oder nur fehlerhaft decodiert worden ist. Ist der Inhalt einer Kodierung fehlerfrei erkannt worden, hat aber länger als eine Sekunde Reaktionszeit benötigt, dann ist dies entsprechend mit einem dritten Status vermerkt worden. Während der Messungen haben sich die QR-Codes hinter einem transparenten Plexiglas befunden und die Smartphones sind immer im Hochformat verwendet worden.

Es sind während der gesamten Messung keine QR-Codes fehlerhaft decodiert worden. Damit sind auch Kodierungen mit einem hohen Informationsgehalt bei einer korrekten Erkennung im Allgemeinen fehlerfrei auswertbar. Interessant ist, dass es eine Korrelation zwischen der Erkennungsrate und der Informationsdichte im Hinblick auf die Blickwinkelstabilität gibt. Ab einer Größe von $5 \mathrm{~cm}^{2}$ werden QR-Codes bei einem vertikalen Blickwinkel von den Smartphones in unterschiedlichen Distanzen zunehmend besser erkannt. Bei einem horizontalen Neigungswinkel ist tendenziell eine höhere Erkennungsrate ab $8 \mathrm{~cm}^{2}$ auf verschiedene Entfernungen zu verzeichnen. Die Markierungen mit geringerem Informationsgehalt, wie die vierstellige und zehnstellige ID sind hingegen sehr anfällig für horizontale sowie vertikale Neigungswinkel. Diesbezüglich sollte zumindest der horizontale Blickwinkel, durch eine nicht zu tiefe und nicht zu hohe Befestigung minimiert werden. Dafür sind diese QR-Codes bei einem direkten Scannen ohne einen Neigungswinkel, gerade bei größeren Distanzen gegenüber den Markierungen mit einer hohen Informationsdichte überlegen. So werden selbst $3 \mathrm{~cm}^{2}$ große QR-Codes von drei der vier Smartphones über eine Distanz von $75 \mathrm{~cm}$ fehlerfrei interpretiert.

\footnotetext{
${ }^{313}$ Sustainable App Developer 2018.
} 
Des Weiteren sollten QR-Codes, die eine hohe Anzahl an Zeichen kodieren nicht zu groß erstellt werden, da diese sonst nicht mehr korrekt von der Kamera des Smartphones erfasst werden können. Gerade bei den aktuelleren Smartphones ist im Test aufgefallen, dass diese nicht nur von den besseren Auflösungen der Hauptkameras, sondern ebenfalls einer öfters stattfindenden Autofokussierung profitieren. Häufig führte eine manuelle Fokussierung der Kamera zu einer Erkennung der Markierungen, wenn auch nicht innerhalb der gewünschten Reaktionszeit von einer Sekunde.

Bezüglich der Fehlerbetrachtung lässt sich anmerken, dass die Auswertung Messfehler durch Ungenauigkeiten in der Bestimmung der Winkel und Längen beinhalten kann. Darüber hinaus können die Bewertung und Erfassung der Reaktionszeit durch den Autor subjektiv gefärbt sein. Abschließend ist anzuführen, dass sich keine durchgehend konstante Raumhelligkeit bei der Durchführung aller Messungen herbeiführen ließ, sodass dies eine weitere mögliche Fehlerquelle sein könnte. Alle ausgewerteten Bögen mit sämtlichen erfassten Messungen sind dem Anhang zu entnehmen. ${ }^{314}$ Darüber hinaus enthält dieser die erstellten QR-Codes für das Testszenario. ${ }^{315}$

Zusammenfassend lässt sich die Aussage treffen, dass QR-Codes ein probates Mittel für die Standortbestimmung im Rahmen eines Pull-Dienstes sind. Wenn die Blickwinkelstabilität und eine hohe Informationsdichte keine Rolle spielen, können sehr kleine Abmaße für die einzusetzenden QR-Codes verwendet werden. Spielen die genannten Faktoren jedoch eine tragende Rolle, so ist eine Größe von mindestens $5 \mathrm{~cm}^{2}$, besser jedoch $8 \mathrm{~cm}^{2}$ zu wählen, um eine hohe Erkennungsrate gewährleisten zu können. Anzumerken ist, dass der QR-Code zur Umsetzung der Anforderung IP 0010 (Positionsbestimmung „Kunst am Bau“) nicht geeignet ist, da er gegen die Anforderung IP 0090 verstoßen würde.

\subsubsection{NFC}

Wie bei QR-Codes, soll gleichermaßen bei der Technologie NFC untersucht werden, wie hoch die korrekte Erkennungsrate bei unterschiedlicher Informationsdichte ist. Von Interesse ist darüber hinaus durch welche Materialien die Verbindung zwischen

\footnotetext{
${ }^{314}$ Pfad: ...Anhang/Evaluation/Test QR.xlsx

${ }^{315}$ Pfad: ../Anhang/Evaluation/Rohdaten/QR
} 
NFC-Tag sowie Smartphone erfolgreich zustande kommt und welche Materialien ein Auslesen der gespeicherten Daten verhindern. Abschließend soll herausgefunden werden wie die maximale Distanz zwischen Sender und Empfänger bei den unterschiedlichen Materialien ist.

Die Messungen sind, wie zuvor bei den QR-Codes hinsichtlich fehlerfreier Interpretation und schneller Reaktionszeit mit drei verschiedenen Status bewertet worden. Im Hinblick auf die Informationsdichte sind die drei unter QR-Code beschriebenen und angewendeten Parameter zum Testen genutzt worden. Eine Varianz hat sich bezüglich der eingesetzten NFC-Tags ergeben. Zum einen sind NTAG 213- und NTAG 215Chips verwendet worden. Die beiden Tags unterscheiden sich marginal in ihrer Größe und dem Speichervolumen. Während sich der NTAG 213 durch einen Durchmesser von $22 \mathrm{~mm}$ und ein Speichervolumen von 142 Byte auszeichnet, weist der NTAG 215 einen Durchmesser von 30 mm und ein Speichervolumen von 540 Byte auf. Zum Kodieren der Testdaten waren die Speichervolumina beider Chipvarianten mehr als ausreichend. Es ist anzumerken, dass das Samsung Galaxy S3 Mini hardwareseitig keinen NFC-Chip integriert hat und deswegen nicht als Testgerät fungieren konnte. Zum Kodieren und Dekodieren der NFC-Tags ist die App „Tag Writer ${ }^{\text {r316 }}$ verwendet worden. Die erfassten Messbögen sind dem Anhang zu entnehmen. ${ }^{317}$

Gesamt sind im Rahmen des Tests 396 Messungen vorgenommen worden. Es lässt sich im Allgemeinen die Aussage treffen, dass sämtliche erfolgreiche Verbindungen zwischen Sender und Empfänger innerhalb der angestrebten Reaktionszeit erfolgt und keine der codierten Daten fehlerhaft interpretiert worden sind. Es ist dabei kein Unterschied im Hinblick auf die Erkennungsrate bei den verschiedenen Chipvarianten festgestellt worden. Lediglich Metall blockiert das Auslesen der Tags, aufgrund seiner abschirmenden Eigenschaften. Durch alle anderen Materialien hindurch kann erfolgreich eine Verbindung zu dem NFC-Tag hergestellt werden. Je nach Dicke des Materials bzw. der Kombination unterschiedlicher Materialien muss das Smartphone näher an den Chip herangehalten werden. Des Weiteren wirkt sich die Nutzung einer TPUbzw. Silikon-Hülle nicht nennenswert auf die Erkennung oder die maximale Distanz aus.

\footnotetext{
${ }^{316}$ NXP Semiconductors 20018.

${ }^{317}$ Pfad: ../Anhang/Evaluation/Test NFC.xlsx
} 
Während des Tests mit unterschiedlichen Geräten ist zu beobachten gewesen, dass die Hardwarekomponente zum Auslesen der NFC-Chips bei den Smartphones an unterschiedlichen Stellen verbaut ist. So ist der NFC-Reader des OnePlus 6 beispielsweise zwischen Hauptkamera und Fingerabdrucksensor im oberen Drittel des Gerätes angesiedelt. Bei den Galaxy-Modellen hingegen sitzt die Hardwarekomponente genau in der Mitte der Smartphones. Da die Distanz zwischen Sender und Empfänger nur sehr gering sein darf, muss der Anwender nach Möglichkeit wissen, wo sich der NFC-Tag ungefähr befindet. Denkbar wäre eine Markierung, die anzeigt, wo der Chip unter der Oberfläche angebracht ist. Alternativ könnten mehrere NFC-Tags mit dem gleichen Inhalt beschrieben werden, um eine größere Fläche ab zu decken, damit Fehlversuche vonseiten des Anwenders minimiert werden.

Laut Angabe des Herstellers sind beide Chips mit einem Schreib- und Passwortschutz versehbar. Dies konnte in diversen Tests bestätigt werden. Zum einen können Daten auf die Chips geschrieben und mit einem Read-only-Merkmal versehen werden. Ein erneutes Überschreiben oder Löschen der NFC-Tags ist dann nicht mehr möglich. Zum anderen kann der Chip vor Manipulation geschützt werden, aber trotzdem wiederbeschreibbar sein, indem die Möglichkeit des Passwortschutzes angewendet wird. Hierbei wird der NFC-Tag mit einem selbst wählbaren Passwort codiert, der ein Überschreiben oder Löschen der gespeicherten Daten nur in Kombination mit dem zugehörigen Passwort erlaubt.

Zusammenfassend betrachtet lässt sich sagen, dass NFC, wie bereits der QR-Code sehr gut zur Umsetzung eines Pull-Dienstes verwendet werden kann. Vorteilhaft ist die 100 prozentige Erkennungs- und Decodierungsrate der NFC-Tags bei kompatiblen Oberflächenmaterial. Darüber hinaus können die Chips visuell unsichtbar in den Standort integriert werden. Diese vollständige Integrität wird jedoch durch die Notwendigkeit eines Hinweises für den Nutzer aufgeweicht. Man hat dafür eine größere gestalterische Freiheit, als bei QR-Codes und kann diese Technologie individueller in ein Designkonzept einbetten.

Nachteilig ist hingegen, wie in Kapitel 4 aufgezeigt, dass nicht jedes Smartphone über die entsprechende Sensorik zum Auslesen der Chips verfügt. Darüber hinaus kann diese Technologie nicht die Anforderung IP0010 (Positionsbestimmung „Kunst am Bau) umsetzen und könnte somit, wie der QR-Code nur einen Teil der 
Standortbestimmung übernehmen. Es wäre demnach eine Kombination mehrerer Technologien, zur vollständigen Umsetzung aller Anforderungen zu präferieren.

\subsubsection{Texterkennung - OCR}

Wie bereits im Ist-Zustand beschrieben, befindet sich das Philologicum noch in der Fertigstellung. Die Kunst am Bau ist eines der letzten zu integrierenden Bauelemente und zum Zeitpunkt des Testes noch nicht vor Ort verfügbar. Deswegen soll die Kunst am Bau mit skalierten Ausdrucken im Maßstab 16:1 der einzelnen Entwurfsmuster im Rahmen der praktischen Tests nachgestellt werden.

Ziel des Testszenarios ist es herauszufinden, mit welcher Präzision die verwendeten Endgeräte die drei selektierten Vorder- und Rückseiten der Texttafeln erkennen können. ${ }^{318}$ Des Weiteren ist es für die spätere Performance des Systems wichtig zu wissen, wie viel Zeit bzw. wie hoch die Anzahl an Scans ist, die für eine korrekte Erkennung benötigt wird.

Zur Umsetzung der Texterkennung ist eine eigene Anwendung „OCR Recognition“319 programmiert worden, deren Quellcode auf der „Google Mobile Vision Text APl“320 basiert. Mithilfe dieser API ist es, unter Verwendung der Kamera eines Smartphones, möglich Texte, auf Basis von lateinischen Schriftzeichen, in Echtzeit zu erkennen und programmatisch weiter zu prozessieren. ${ }^{321}$ Basierend auf der Struktur eines Textes kann die Anwendung automatisiert verschiedene Elemente identifizieren und auslesen.

Wie Abbildung 27 visualisiert segmentiert die Programmbibliothek der Mobile Vision API einen Text in unterschiedliche Bausteine. Die Gesamtheit eines Abschnittes wird als „Blocks“ bezeichnet. Dieser wiederrum besteht aus einzelnen „Lines“, eine kontinuierliche Ansammlung von „Words“ auf derselben vertikalen Ebene. Die elementaren Komponenten „Words“ bilden schließlich das Fundament eines Textes. ${ }^{322}$ Eine Optical

\footnotetext{
${ }^{318}$ Die verwendeten Texttafeln sind dem Anhang beigefügt.

Pfad: ../Anhang/Evaluation/Rohdaten/OCR

${ }^{319}$ Der Quellcode für die App „OCR Recognition“ kann dem Anhang entnommen werden.

Pfad: ../Anhang/App/OCRRecognition/

${ }^{320}$ Google LLC 2018h.

${ }^{321}$ Vgl. Google LLC 2017.

${ }^{322}$ Vgl. Google LLC 2017.
} 
Character Recognition (OCR)-Erkennung ist prinzipiell auf jeder strukturellen Ebene möglich und über die API abbildbar.
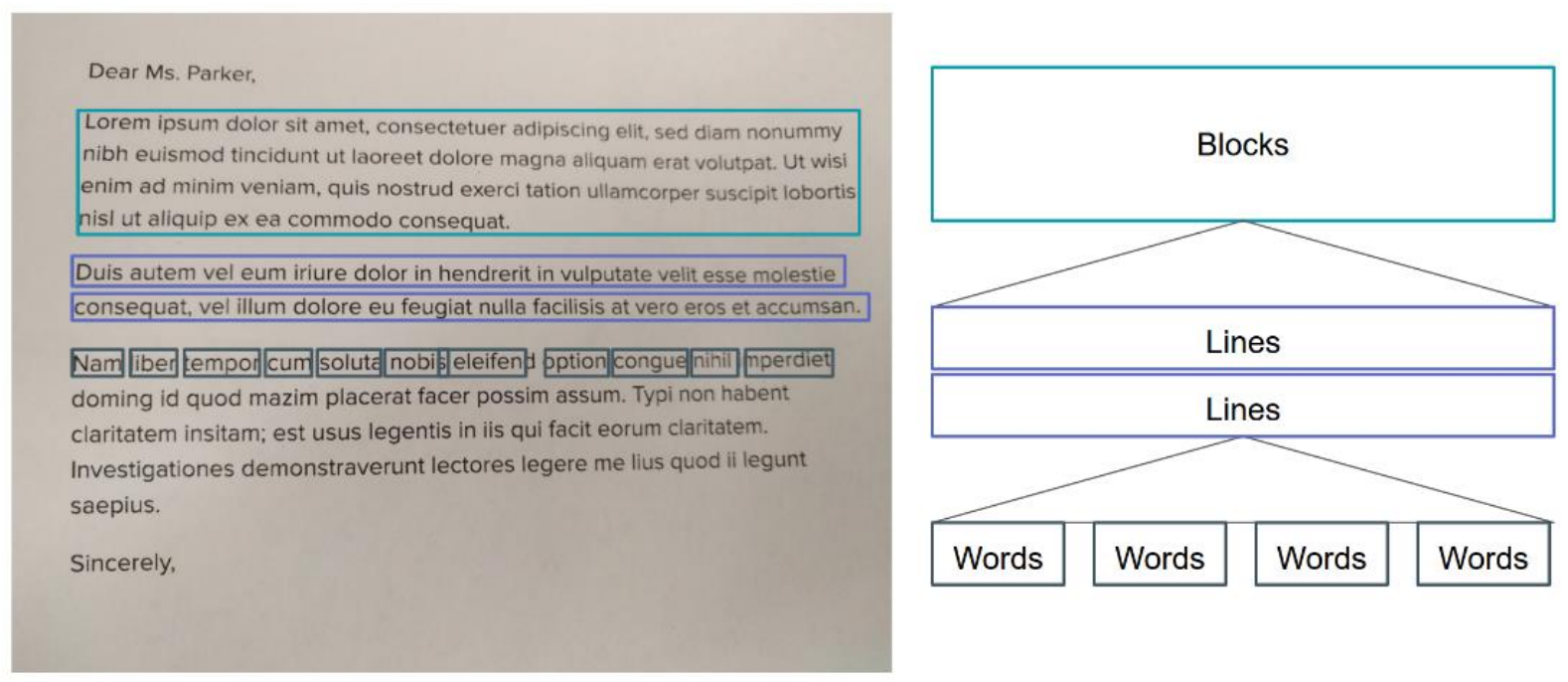

Abbildung 27: Strukturelemente eines Textes der Mobile Vision AP ${ }^{323}$

Im Zuge der App „OCR Recognition“ und damit innerhalb des Testszenarios ist der Grundgedanke, einzelne Textpassagen aus den Beispieltafeln zu erkennen und einem Standort zu zuordnen. Dafür ist es notwendig eindeutige Schlüsselwörter bzw. Kombination von Schlüsselwörtern aus den Texten zu extrahieren, die im Zuge der Zitatsammlungen einmalig sind. Diese Schlüsselwörter werden in der Anwendung als Basis für die Vergleiche und folglich der Zuordnung zu einem Standort hinterlegt. Im Hinblick auf die drei unterschiedlichen strukturellen Erkennungsstufen der Mobile Vision API ist das Element „Lines“ für die Texterkennung ausgewählt worden, da es den besten Kompromiss aus Erkennungsrate und Vergleichbarkeit abbildet. Das Element „Block“ ist für einen Vergleich zu umfangreich und die Erkennungsrate würde wahrscheinlich darunter leiden. Das kleinste Element „Words“ wiederum würde die korrekte Zuordnung bzw. die Integrität im Sinne der Eindeutigkeit gefährden.

Erkennt die App nun eine Textzeile vergleicht Sie diese, mit den in der Anwendung zuvor hinterlegten Schlüsselwörterkombinationen. Bei einer Übereinstimmung kann eine Zuordnung zu einem Standort, in Form einer ID erfolgen. Im Prinzip handelt es sich um einen klassischen Fingerprinting Algorithmus. Die Signaturen bilden die konkreten Kombinationen der Schlüsselwörter, die in einer Trainingsphase eingesammelt werden müssen.

\footnotetext{
${ }^{323}$ Quelle: Google LLC 2017.
} 
Abbildung 28 zeigt eine Beispieltafel und die darauf enthaltenen Zitate. Die Textzeile „Miss Broke had" ist beispielsweise als Schlüsselwörterkombination in der Anwendung für den Standort mit der ID 11 hinterlegt worden. Erkennt die Anwendung diese Textzeile kann somit über einen Vergleich erfolgreich die aktuelle Position bestimmt werden.

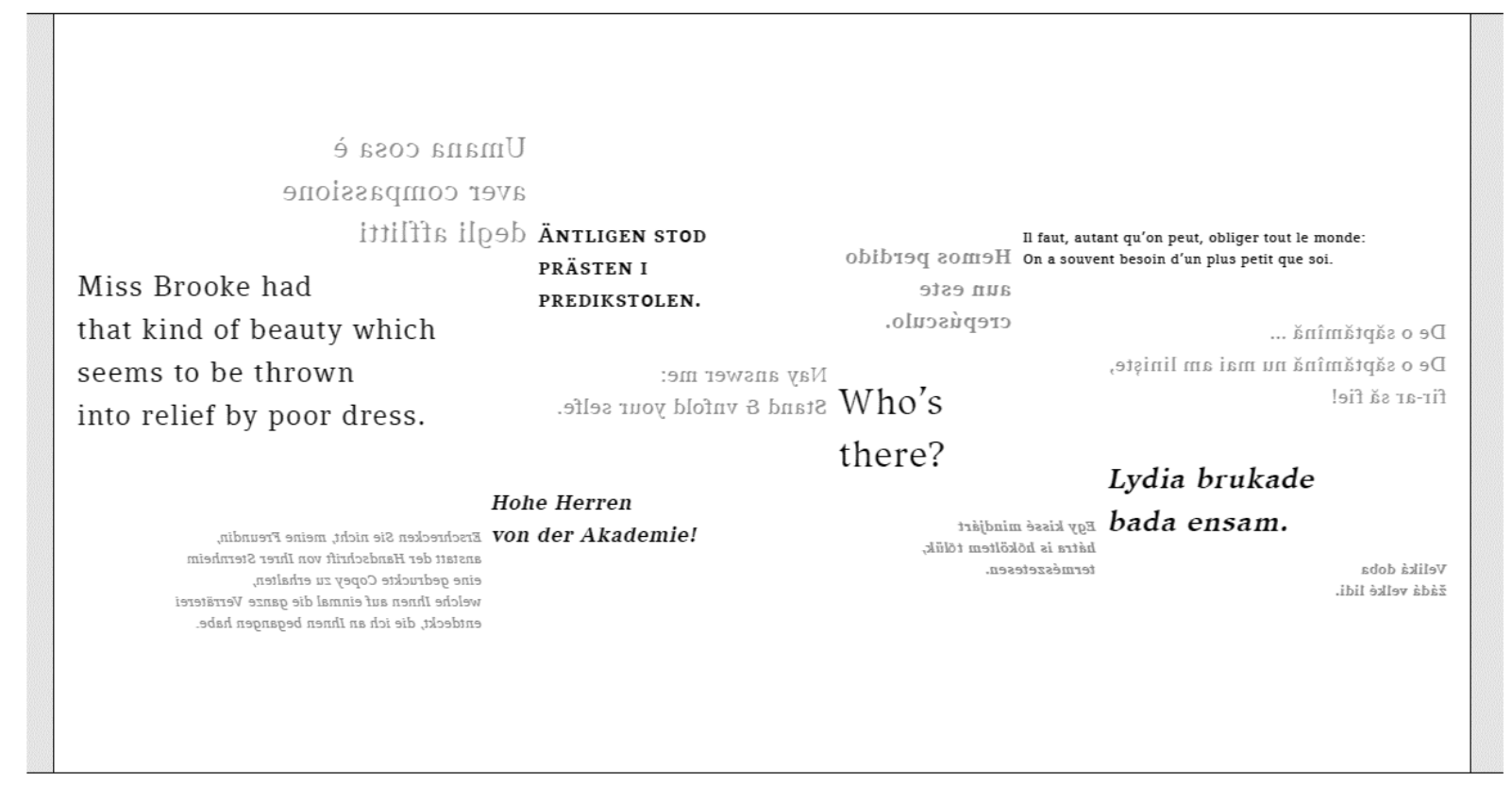

Abbildung 28: Beispiel „Kunst am Bau“ im Philologicum ${ }^{324}$

Im Rahmen des Testszenarios ist jede Textzeile, der zu untersuchenden Tafeln in originärer Form in der App „OCR Recognition“ hinterlegt und einem korrelierenden Standort zugeordnet worden. ${ }^{325}$ Auf Grundlage der Spezifikationen und der Einschränkung der Mobile Vision API ist es zudem interessant herauszufinden, wie sich die Schnittstelle verhält, wenn ein Text erkannt wird, der nicht auf lateinischer Schrift beruht, da einige Texttafeln der Kunst am Bau dem kyrillischen oder griechischen Alphabet entspringen. Die verwendeten Tafeln enthalten demnach unter anderem Beispiele der russischen und griechischen Schrift.

Die Testreihe hat für jedes der verwendeten Endgeräte mehrere hundert Messergebnisse hervorgebracht. Gesamt betrachtet ist die Erkennungsrate von lateinischer Schrift über alle Smartphones hinweg hervorragend und eine Zuordnung zu einem Standort kann in der Regel innerhalb kürzester Zeit erfolgen. Beeindruckend ist gleichfalls das Verhältnis aus Scans und erfolgreichen Vergleichen mit den hinterlegten

\footnotetext{
${ }^{324}$ Quelle: Eigene Darstellung, auf Basis der Bau- und Etagenpläne des Philologicums.

${ }^{325}$ Die gesamte Zuordnung der Kombinationen aus Schlüsselwörtern zu den jeweiligen Standort-ID's ist dem Quellcode der App „OCR Recognition“ zu entnehmen.
} 
Schlüsselwörtern. Es ist keine Seltenheit, dass innerhalb eines Scans mehrmals erfolgreich eine Position bestimmt werden kann.

Es hat sich herausgestellt, dass gerade moderne Hardware, wie bei dem Testgerät OnePlus 6, innerhalb einer Sekunde gleich mehrere Scans erfassen, prozessieren und auswerten kann. Damit steigt die Wahrscheinlichkeit einer raschen Erkennung von Kombinationen aus Schlüsselwörtern enorm an. Aber selbst Geräte der Einstiegsklasse, wie das ZTE Blade 5 werten einzelne Scans in einem zeitlichen Abstand von etwa 2 Sekunden aus und erkennen zuverlässig die hinterlegten Textsamples.

Bezüglich der Verarbeitungsgeschwindigkeit eines Scans ist in der App ein Optimierungspotential, vor allem bei einer späteren Verwendung für einen Prototypen, erkannt worden. Momentan zeichnet die App bei der Verwendung den erkannten Text auf dem Display des Smartphones nach und rendert diesen. Dieser Arbeitsschritt der Visualisierung kann zu einem späteren Zeitpunkt entfallen und bindet folglich keine Ressourcen mehr. Es ist anzunehmen, dass trotz einer größeren Anzahl an Vergleichen, durch die Hinterlegung aller Schlüsselwortkombinationen, die Performance ohne die Visualisierung steigen wird.

Interessant ist die Beobachtung gewesen, dass nicht lateinische Schrift von den Endgeräten trotzdem ausgelesen, aber eben nicht in der korrekten Schrift extrahiert wird, da der Zeichensatz offenbar unbekannt ist. Ein Beispiel hierfür sind die Auswertungen der Rückseite von Beispieltafel 2. Hier hat nur das OnePlus 6, aufgrund seiner raschen Auswertung und somit der enormen Anzahl an Scans eine korrekte Zuordnung durchgeführt.

Es wäre jedoch denkbar, den Algorithmus zur Texterkennung so anzupassen, dass nicht etwa immer eine gesamte Zeile korrekt erkannt werden muss, sondern auch einzelne übereinstimmende Bestandteile zu einem erfolgreichen Vergleich führen können. Darüber hinaus kann eine Speicherung von modifizierten Schlüsselwortformen oder Kombinationen von diesen, ebenfalls einen positiven Effekt für einen erfolgreichen Vergleich liefern. Als logische Schlussfolgerung lässt sich subsummieren, dass je größer die Basis an eindeutigen Schlüsselwortkombinationen, desto höher ist die Wahrscheinlichkeit einer raschen und erfolgreichen Erkennung sowie Zuordnung zu einem Standort.

Empfehlenswert ist daher, verschiedene Smartphones im Rahmen der initialen Trainingsphase des Systems, zur Sammlung von eindeutigen Kombinationen von 
Schlüsselwörtern zu verwenden. Vorteilhaft ist, dass dann neben den originären auch modifizierte und von den Geräten erkannte Wortformen in die Vergleichsbasis Einzug halten. Mit diesem breiten Fundament an Vergleichsoptionen und Zuordnungen zu verschiedenen Standorten, kann eine effizientere Standortbestimmung gewährleistet werden.

Anzumerken ist, dass im Verlauf der Programmierung der Anwendung bereits die Erprobung eines Filterschrittes durchgeführt worden ist. Die Idee ist es gewesen, dass eine Textzeile in zwei aufeinanderfolgenden Scans erkannt werden muss und anschließend erst der Vergleich mit den Schlüsselwörtern stattfinden sollte. Dieser Filter ist als eine Art Verifizierung der gescannten Inhalte gedacht gewesen und sollte die Anzahl der Vergleiche reduzieren. Darüber hinaus könnte bei einer großen Anzahl an Kombinationen verschiedener Schlüsselwörter, die eindeutige Zuordnung zu einem Standort leiden. Jedoch hat sich im Laufe der Analyse herausgestellt, dass dieser Schritt obsolet ist und die erfolgreiche Positionsbestimmung nur verzögert. Gerade bei Smartphones mit einer tendenziell leistungsschwachen Hardware führt dieser Verifizierungsschritt zu einer unnötigen Verlangsamung.

Sollte ein Verifizierungsschritt im produktiven Betrieb notwendig sein, dann ist dieser anders zu wählen. Im Sinne der Prävention einer falschen Positionsbestimmung und damit einer gewissen Robustheit kann dieser jedoch sinnvoll sein. Beispielsweise könnte geprüft werden, wie oft innerhalb eines oder mehrerer aufeinanderfolgender Scans eine bestimmte Standort-ID zugeordnet worden ist. Die am häufigsten vergebene ID kann anschließend als zu bestimmende Position verwendet werden. Denkbar wäre auch, dass wenn mehrere Standorte in Frage kommen, der Nutzer ein kurzes Feedback im Sinne von „Das ist nicht die richtige angezeigte zusätzliche Information!“ abgeben kann. Anschließend könnte die nächst häufige und damit wahrscheinlichere Standort-ID zur Positionsbestimmung verwendet werden.

Zusammenfassend betrachtet lässt sich über OCR, als optische und visuelle Technologie zur Positionsbestimmung sagen, dass diese für die Umsetzung der Anforderung IP 0010 (Positionsbestimmung „Kunst am Bau), mit allen korrelierenden Anforderungen sehr gut geeignet ist und für eine Weiterführung im Zuge der Konzeption empfohlen wird. Des Weiteren ist anzumerken, dass auch die Anforderung IP 0020 (Positionsbestimmung Raumreservierungssystem, technische Geräte, Freihandbestand) mit dieser Technologie umsetzbar ist. Die User Story zur Raumreservierung könnte über 
ein Auslesen der Raumnummer realisiert werden, da die Anwendung bereits im Test erfolgreich Nummern aus den Beispieltafeln extrahiert hat. Für die restlichen verbleibenden Userszenarien ist ebenfalls eine Umsetzung mit OCR realisierbar. Gerade im Vergleich zu QR-Codes ist eine größere Gestaltungsfreiheit der Markierung von Vorteil, da sich Text als identifizierendes Merkmal individueller gestalterisch verarbeiten lässt. Diese Flexibilität in der Umsetzbarkeit ist ein weiteres positives Indiz für eine Verwendung der Technologie hinblickend auf die Konzeption des Systems.

Abschließend verleibt noch zu nennen, dass alle ausgewerteten Bögen, mit sämtlichen erfassten Messungen dem Anhang zu entnehmen sind. ${ }^{326}$ Es ist zu beachten, dass aufgrund der Komplexität der erfassten Daten pro Endgerät separate Datenblätter gespeichert worden sind.

\subsection{Ergebnis Evaluation und praktische Tests}

Nach Abschluss der mehrstufigen Evaluation sowie den praktischen Testszenarien hat sich klar die optische und visuelle Technologie der Texterkennung - OCR gegenüber anderen Technologien im Anwendungsszenario der Auftraggeberin durchgesetzt. Zum einen kann sie alle aufgestellten nichtfunktionalen Anforderungen im Hinblick auf Indoor Positioning umsetzen und zum anderen die genannten Funktionen der User Stories in gleicher Weise bedienen.

Diese Technologie und OCR im speziellen bringen gegenüber anderen Technologien zahlreiche Vorteile mit sich. Einer der größten Vorteile ist, dass jedes Smartphone auf dem heutigen Markt über eine Kamera verfügt. Es wird somit kein weiterer Sensor benötigt, der explizit durch den Nutzer aktiviert oder dessen Berechtigung eingeholt werden müsste. Im Hinblick auf Android wird zum Beispiel lediglich der initiale Zugriff auf die Hardwareressource der Kamera angefordert. Andere Technologien zur Positionsbestimmung sind dahingehend nicht so restriktiv, sondern fordern eine umfangreiche Einräumung von Zugriffsrechten. Darüber hinaus ist die Verwendung der Kamera als Hauptsensor im Vergleich zu anderen Technologien, die zusätzliche Sensorik benötigen, überaus stromsparend.

\footnotetext{
${ }^{326} \mathrm{Pfad}:$../Anhang/Evaluation/Test OCR [Gerätename].xIsx
} 
Damit einher geht, zumindest für die Standortbestimmung, eine unkomplizierte Handhabung des Datenschutzes. Die gesamte Prozessierung kann in der Anwendung des Smartphones stattfinden, ohne das datenschutzrechtlich bedenkliche Daten dieses verlassen müssen. Des Weiteren stellt Google mit seiner Mobile Vision API ein unkompliziertes Framework zur Verfügung, das auch für den Einsatz auf iOS zur Verfügung steht. ${ }^{327}$ Eine Portierung auf iOS ist folglich auf die Positionierung nicht erschwert, sondern kann vielmehr problemlos adaptiert werden. Interessant ist anzumerken, dass das Framework die Google Play Services nutzt und damit eine Entkopplung vom API-Level des Android OS stattfindet. ${ }^{328}$ Vorteilhaft ist, dass dieses Framework unabhängig von der Versionierung des OS auf jedem Android Gerät, dass den Google Playstore nutzt, zur Verfügung steht.

Wie die praktischen Tests gezeigt haben, ist die Texterkennung eine performante Technologie, die sich bei einer gut kalibrierten Trainingsphase durch eine hohe Genauigkeit und Präzision auszeichnet. Im eigentlichen Sinn handelt es sich hierbei um einen klassischen Fingerprinting Algorithmus, der bei softwareseitigen Anpassungen individuell und flexibel erweiterbar ist. Vorteilhaft ist, dass nach initialer Erfassung aller Signaturen kein bzw. nur ein marginaler Wartungsaufwand entsteht, da sich in dem Anwendungsszenario die Signaturen (Kombination der Schlüsselwörter) nicht ändern, sondern maximal neue hinzukommen. Bei anderen Technologien müssten in regelmäßigen Intervallen alle Signaturen aktualisiert werden, damit die Standortbestimmung dauerhaft präzise funktioniert. Auch sind keine zusätzlichen elektronischen Geräte notwendig, die extra gewartet werden müssten.

Immanent mit der Kamerasensorik verbunden ist überdies hinaus, dass auf unterschiedlichsten Endgeräten nahezu identische Messwerte, gerade im Vergleich zu Magnetismus, WLAN oder BT aufgezeichnet werden. Dies bedingt sich durch die weitest gehende Unabhängigkeit von »Störeffekten«. Dadurch wird eine einheitliche Prozessierung, Analyse und Adaptierbarkeit auf verschiedenen Smartphones enorm begünstigt. Eine Einbindung der entsprechenden Hardwareressource in die mobile Anwendung ist, beispielsweise bei Android unproblematisch, da bereits im API-Level 1 die entsprechenden Pakete zur Nutzung bereitgestellt werden.

\footnotetext{
${ }^{327} \mathrm{Vgl}$. Google LLC $2018 \mathrm{e}$.

${ }^{328}$ Vgl. Google LLC 2018g.
} 
Innerhalb des optischen bzw. visuellen Technologiezweiges ist OCR, im Vergleich zu QR-Codes, individueller in den physischen Raum integrierbar. Begünstigt durch die Tatsache, dass Text als Markierung für dieses Verfahren verwendet wird, ist es für den Nutzer unauffällig, dass es sich um eine Kennzeichnung zur Standortbestimmung handelt. Folglich wird in der Regel die visuelle Einheit durch diese Technologie erhalten bleiben können.

Durch die Abbildung aller Anforderungen mit nur einer Technologie, ohne eine Kombination mehrerer zu verwenden, ist mit einer Reduzierung der Komplexität des zu konzipierenden Systems zu rechnen. Dies ist laut Anforderungsanalyse im Sinne der Auftraggeberin.

Von Nachteil ist, dass zur Standortbestimmung eine Sichtlinie zwischen Endgerät und der zu erkennenden visuellen Einheit benötigt wird. Jedoch wird dieser Nachteil durch den Fakt, der Notwendigkeit einer visuellen Rezeption, zum Beispiel der Kunst am Bau“, durch den Nutzer aufgeweicht. Auch die anderen ortsabhängigen User Stories bedingen eine Sichtlinie des Nutzers zwischen inm und dem einzelnen Objekt, mit dem interagiert werden soll.

Bezüglich der vollständig individuellen Integration der Technologie in den physischen Raum und der eventuell fehlenden Wahrnehmung der Nutzer als visuelle Markierung für eine Positionsbestimmung, ist es möglich, dass dieser den POI als solchen nicht wahrnimmt und er möglicherweise übersehen wird. Hier ist es empfehlenswert sich über eine optische Auszeichnung zur Erhöhung des Wiedererkennungswert konzeptionelle Gedanken zu machen, damit dem Anwender unauffällig, aber deutlich signalisiert wird, dass an einem bestimmten Standort Interaktionsmöglichkeiten bestehen. Des Weiteren ist ein initialer Aufwand zum Bestimmen der Schlüsselwörter bzw. geeigneter Kombinationen dieser notwendig, um eine effektive Standortbestimmung durchführen zu können. Dieser Initialisierungsaufwand ist jedoch auch bei anderen Technologien notwendig und geht immer im Rahmen der Verwendung eines Fingerprinting Algorithmus einher.

Abschließend ist bezüglich der Nachteile noch die Skalierbarkeit zu nennen. Sollte das System auf andere Standorte erweitert werden, dann muss sich die Adaption gleichfalls an dieser optischen Technologie ausrichten und entsprechend konzipiert werden. Andererseits ist anzumerken, dass der Skalierbarkeit in der Prioritätsanalyse eine nachrangige Rolle zugeordnet worden ist. 
Aggregiert man Vor- und Nachteile und wiegt diese gegeneinander ab, so ist deutlich zu erkennen, dass die Vorteile offenkundig überwiegen. Es spricht kein gravierender Nachteil gegen eine Verwendung der Technologie im Rahmen der Konzeption und prototypischen Umsetzung. Somit wird OCR als Positionierungstechnologie für das Frontend verwendet. 


\section{Konzeption des Systems}

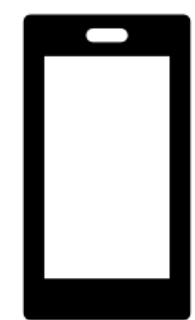

Frontend

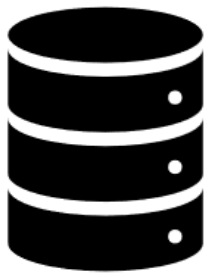

Middleware

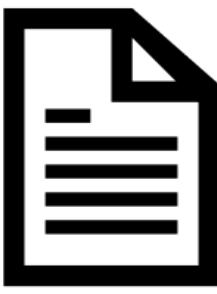

Backend

Abbildung 29: Gesamtarchitektur IPS ${ }^{329}$

Basierend auf den Erkenntnissen des Kapitels 3 "Location-based Services" und Kapitels 6 „Anforderungs- und Zielgruppenanalyse“ lässt sich das zu konzipierende System in insgesamt drei Komponenten strukturieren. Jedes Element übernimmt einen eigenständigen Teil der funktionalen sowie nicht funktionalen Anforderungen und bündelt diese jeweils in einer separaten Funktionseinheit. Die evaluierten Informationen der Anforderungsanalyse und der Testszenarien behalten somit weiterhin ihre Gültigkeit. Sie sind als Grundlage der Konzeption zu betrachten. Dies gilt vor allem für die zu verwendende Positionierungstechnologie.

Die Kommunikation zwischen den einzelnen Elementen des Systems soll via APIs realisiert werden, damit eine Flexibilität bezüglich der Austauschbarkeit der Komponenten gewährleistet ist. Vorteilhaft ist dies, wenn beispielsweise im späteren Produktivbetrieb das Frontend für verschiedene OS angeboten werden soll. Die angestrebte Modularisierung des Systems soll neben der Interoperabilität, gleichermaßen die zukünftige Erweiterbarkeit und Wartungsfähigkeit erleichtern.

Die Konzeption des Systems beschäftigt sich in diesem Kapitel mit den zwei Komponenten Frontend und Middleware. Wie bereits in der Anforderungsanalyse eruiert, wird

\footnotetext{
${ }^{329}$ Quelle: Eigene Darstellung.
} 
als Backend das bestehende CMS, auf Basis von „Fiona“ ${ }^{\text {‘330 }}$, der Auftraggeberin verwendet und soll dahingehend nicht Bestandteil der Konzeption sein. Eine Kommunikation zwischen Middleware und Backend ist nicht vorgesehen, da diese beiden Elemente als eigenständige Funktionseinheiten unabhängig voneinander agieren sollen. Vielmehr verquickt das Frontend beide Systeme miteinander und aggregiert dessen gespeicherte Daten.

Grundlage für den Entwurf des LBS ist die Aufteilung des Philologicums in unterschiedliche POls. Jedem POI und damit Standort, an dem zusätzliche Informationen angeboten werden sollen, ist eine eindeutige Location-ID zugeordnet. Die Verknüpfung zwischen beiden Elementen sollte zur Wahrung der Übersichtlichkeit und Integrität festgeschrieben werden. Dies kann zum Beispiel in analoger Form auf den Bauund Etagenplänen stattfinden oder wäre zukünftig, bei Ausbau der Middleware, wie in Kapitel 8.2 aufgezeigt, gleichermaßen digital möglich. Die Planung und Umsetzung aller POls im Philologicum ist nicht Bestandteil dieser Arbeit, sondern kann im Nachhinein in dessen praktischer Umsetzung für den Produktivbetrieb erfolgen.

Eine weitere Grundlage für das System ist die Arbeit mit Content-IDs, die eine eindeutige Zuordnung zum Inhalt des CMS ermöglichen. Das von der UB genutzte System verwendet diese in Form von eindeutigen Nummern. Zur beispielhaften Darstellung werden beide IDs im Folgenden zur Veranschaulichung genutzt.

Eine logische Schlussfolgerung der Anforderungsanalyse ist die Verwendung eines strukturierten Datenformates, wie Extensible Markup Language (XML) oder JavaScript Object Notation (»JSON«) gewesen. Wenn Daten über die API zwischen den einzelnen Komponenten des Systems übertragen werden sollen, dann ist ein standardisiertes Datenformat, dass alle Komponenten abrufen und auswerten können unumgänglich. Die genauen Spezifikationen können jedoch zunächst in der prototypischen Umsetzung getroffen werden, da diese für die Konzeptionierung zu speziell sind. Möglicherweise sind noch weitere detaillierte Änderungen und Anpassungen im Zuge der Umsetzung im Produktivbetrieb notwendig, die zum Zeitpunkt der Konzeptionierung nicht ersichtlich gewesen sind.

\footnotetext{
${ }^{330}$ Infopark AG.
} 


\subsection{Konzeption des Frontends}

Das Frontend nimmt, wie bereits in der Gesamtarchitektur ersichtlich, eine besondere Rolle innerhalb des Systems ein. Es ist die zentrale Komponente, die neben der Standortbestimmung als Hauptaufgabe, noch zahlreiche weitere verknüpfende und aggregierende Funktionen übernimmt. Zur besseren Darstellung sowie Erhöhung der Anschaulichkeit sollen in diesem Kapitel anhand der prototypischen Graphical User Interface (GUI) Funktionen und Workflows sowie das Zusammenspiel mit den anderen Systemkomponenten erläutert werden.

Wichtig ist anzumerken, dass zur Umsetzung des Konzeptes keine Ausführungen gemacht werden. Diese sind, für ausgewählte Szenarien im Hinblick auf Frontend und Middleware, im Kapitel 9 „Prototypische Umsetzung LMU Spot“ zu finden. Des Weiteren ist zu beachten, dass, wie bereits in der Anforderungsanalyse beschrieben, der Fokus auf den ortsabhängigen Funktionen liegt.

\subsubsection{Frontend GUI - Gesamtüberblick}

Die GUl ist mit dem Tool „Adobe XD CC ${ }^{\star 331}$ entworfen worden. Es handelt sich um ein kostenfreies Programm der Firma Adobe, dass auf die Erstellung von Prototypen und Mockups für unterschiedliche Plattformen ausgelegt ist. Da der Prototyp für die Plattform Android entworfen werden soll, ist das entsprechende User Interface (UI)$\mathrm{Kit}^{332}$ des Material Designs verwendet worden. Komponenten, wie Slider oder Buttons sind daher dem Design von Android angepasst. Der vollständige Entwurf und ein Demonstrationsvideo sind dem Anhang zu entnehmen. ${ }^{333}$

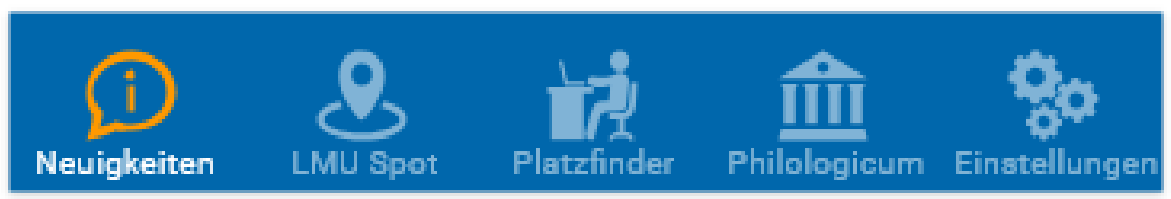

Abbildung 30: Bottom-Navigation-Bar der App „Philologicum ${ }^{\star 334}$

\footnotetext{
${ }^{331}$ Weitere Informationen über das Tool unter: https://helpx.adobe.com/de/xd/how-to/what-is-xd.html

${ }^{332}$ Adobe Systems Software Ireland Limited 2018.

${ }^{333}$ Adobe XD-Entwurf: Pfad: ../Anhang/Ul/Prototyp.xd

Demonstrationsvideo: Pfad: ../Anhang/UI/Prototyp.mp4

${ }^{334}$ Quelle: Eigene Darstellung.
} 
Aus den User Stories, Personas und funktionalen sowie nicht funktionalen Anforderungen hat sich ergeben, das UI in fünf verschiedene Hauptbildschirme zu unterteilen. Wie in den Richtlinien des Material Designs vermerkt, bietet sich für diese Anzahl an Hauptnavigationspunkten eine Bottom-Navigation-Bar an, mit der der Nutzer durch die einzelnen Bildschirme der Anwendung navigieren kann und die immer präsent ist. $^{335}$ Die verwendeten Icons entstammen der Icon-Suchmaschine „Iconmonstr ${ }^{6336}$ und können zur freien, selbst kommerziellen Nutzung verwendet werden. ${ }^{337}$ Im Sinne der Barrierefreiheit sollten die Icons, bzw. die Einheiten aus Bild und Schrift, mindestens eine Größe von 48x48 Pixeln aufweisen. ${ }^{338}$ Wie in Abbildung 30 dargestellt, ist bezüglich Anwendungsfarbgebung das Corporate Design der UB der LMU beachtet worden. ${ }^{339}$

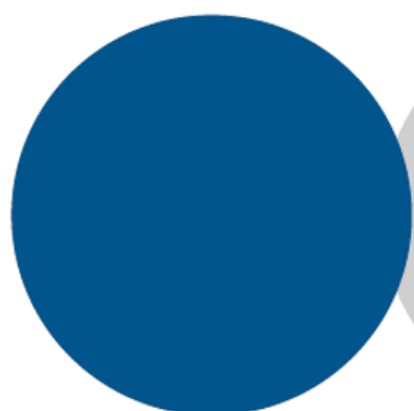

RGB 0/103/172

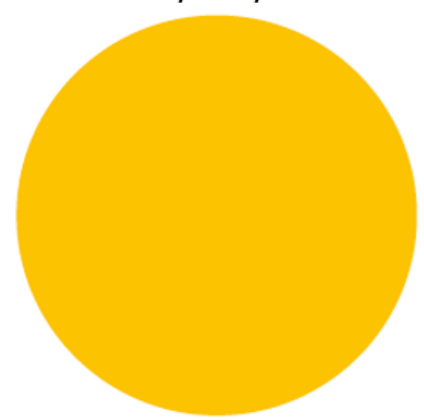

RGB $228 / 187 / 25$

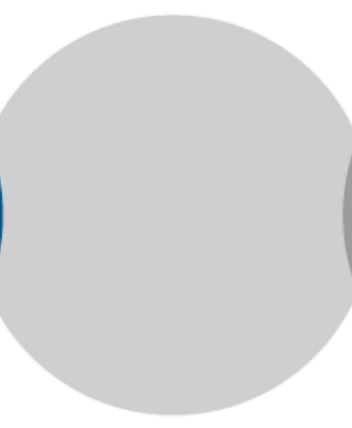

RGB 147/149/152

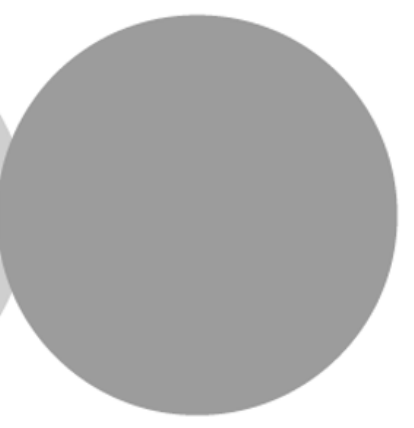

RGB 35/31/32

Abbildung 31: Farbkonzept UB der LMU ${ }^{340}$

Im wesentlich basiert dieses auf vier verschiedenen Farbakzenten, wie in Abbildung 31 dargestellt. Die Farben finden sich in dem Prototyp an unterschiedlichen Stellen

\footnotetext{
${ }^{335} \mathrm{Vgl.}$ Google LLC 2018b.

${ }^{336}$ Kahlkopf 2018a.

${ }^{337}$ Vgl. Kahlkopf 2018b.

${ }^{338} \mathrm{Vgl}$. Google LLC $2018 f$.

${ }^{339}$ Die exakten Farbwerte und weitere detaillierte Angaben sind dem Corporate Design zu entnehmen. Pfad: ../Anhang/Corporate Design/

${ }^{340}$ Quelle: Eigene Darstellung auf Basis des Corporate Design UB der LMU.
} 
wieder. Auf die Verwendung von zusätzlichen Farben ist verzichtet worden, damit der Wiedererkennungswert als ein Produkt der UB erhalten bleibt.

Abschließend ist anzumerken, dass im Rahmen der Konzeptionierung keine umfangreichen Design- oder Usabilitystudien durchgeführt worden sind, um die bestmögliche GUI zu entwerfen. Vielmehr sollen diese den Anforderungen der Auftraggeberin gerecht werden sowie die im Anforderungskatalog aufgeführten Punkte in funktionaler Art und Weise bedienen. Die konzeptionierte GUI soll als Grundlage für die zukünftig zu entwickelnde App dienen und eine Orientierungsgrundlage bieten.

\subsubsection{Frontend - Neuigkeiten}
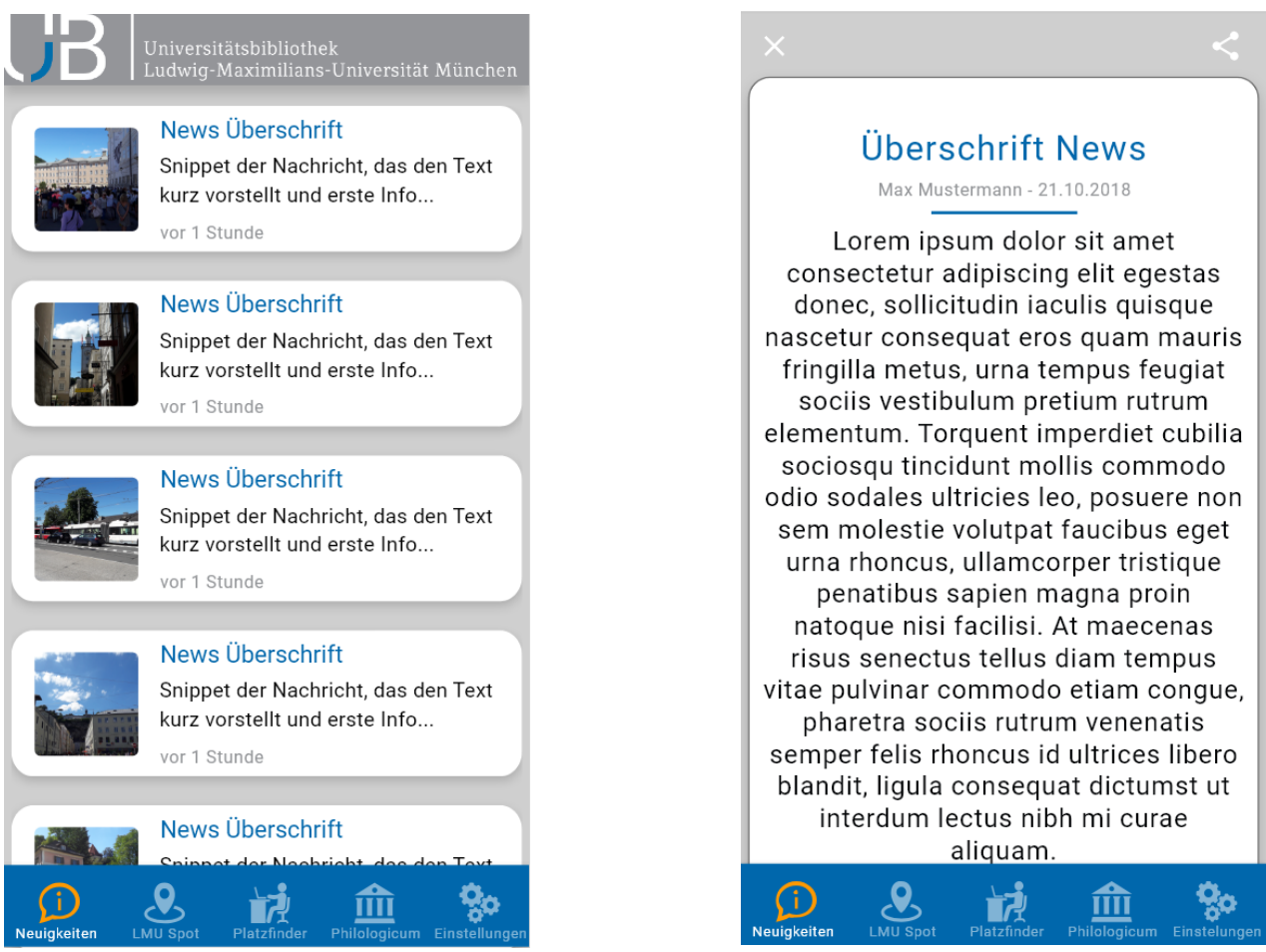

Abbildung 32: Übersicht Neuigkeit und Detailansicht ${ }^{341}$

Bei den Neuigkeiten handelt es sich um eine ortsunabhängige Komponente, die über aktuelle Ereignisse rund um das Philologicum informieren soll. Die Übersicht aller Nachrichten wird, wie in Abbildung 40 gezeigt, als einzelne Kacheln geladen und enthalten neben einem Preview-Bild, Überschrift, Snippet zum Inhalt der Meldung ebenfalls einen Zeitpunkt, wann diese Nachricht veröffentlicht worden ist. Bei initialem Öffnen der Anwendung werden die gültigen Neuigkeiten von der Anwendung geladen.

\footnotetext{
${ }^{341}$ Quelle: Eigene Darstellung.
} 
Zur Aktualisierung kann der Nutzer den Bildschirminhalt nach unten ziehen. Diese Geste ist dem Nutzer als Standard bei gängigen mobilen OS bekannt.

Bei Antippen der einzelnen Information soll dem Benutzer eine Detailansicht präsentiert werden, die den Inhalt der Nachricht enthält. Daneben werden ihm zusätzliche Informationen, wie der Verfasser und Zeitpunkt der Neuigkeit angezeigt. Neben der Hauptnavigation über die Bottom-Navigation-Bar kann ebenfalls über das Kreuz in der linken oberen Ecke zur Übersicht zurückgekehrt werden. Wie bei allen Detailansichten hat der Anwender darüber hinaus die Möglichkeit, den Inhalt der mobilen Anwendung über den „Share“-Button, oben rechts mit anderen zu teilen.

Der grundsätzliche Workflow, zum Abrufen der Informationen kann über die API zwischen Front- und Backend realisiert werden. Die Einbindung der Middleware ist nicht zwingend erforderlich. Vielmehr können alle Informationen direkt vom Backend abgerufen werden. Die Inhalte sind im CMS der UB bereits vollständig enthalten und müssen in der Anwendung lediglich an die mobile Ansicht angepasst werden.

\subsubsection{Frontend - LMU Spot}

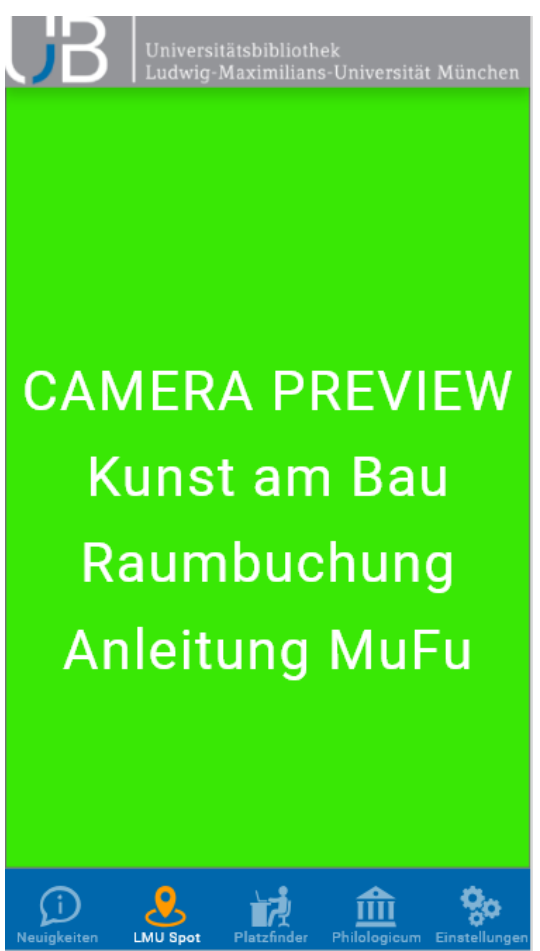

Abbildung 33: LMU Spot ${ }^{342}$
Der Bereich „LMU Spot“ stellt das ortsabhängige Kernelement der Anwendung dar. In diesem Bildschirm werden alle standortbasierten Informationen und Detailansichten der weiterführenden Informationen gebündelt. Wie in Kapitel 7 „Evaluation und Testszenarien“ eruiert, soll die Positionsbestimmung mittels OCR auf Basis der Kamera erfolgen. Der Anwender scannt demnach verschiedene Texte mithilfe der App ein und erwartet zusätzliche Informationen zu diesen.

Abbildung 33 zeigt die Hauptansicht für die Funktion LMU Spot. Bis zur erfolgreichen Positionsbestimmung wird eine Kamera-Preview angezeigt, an dieser Stelle symbolisiert durch die grüne Fläche.

\footnotetext{
${ }^{342}$ Quelle: Eigene Darstellung.
} 
Je nach identifiziertem Standort wird sich einer der drei Detailansichten für die User Szenarien „Kunst am Bau“, „Raumbuchung“ oder „Anleitung technische Geräte“ öffnen.
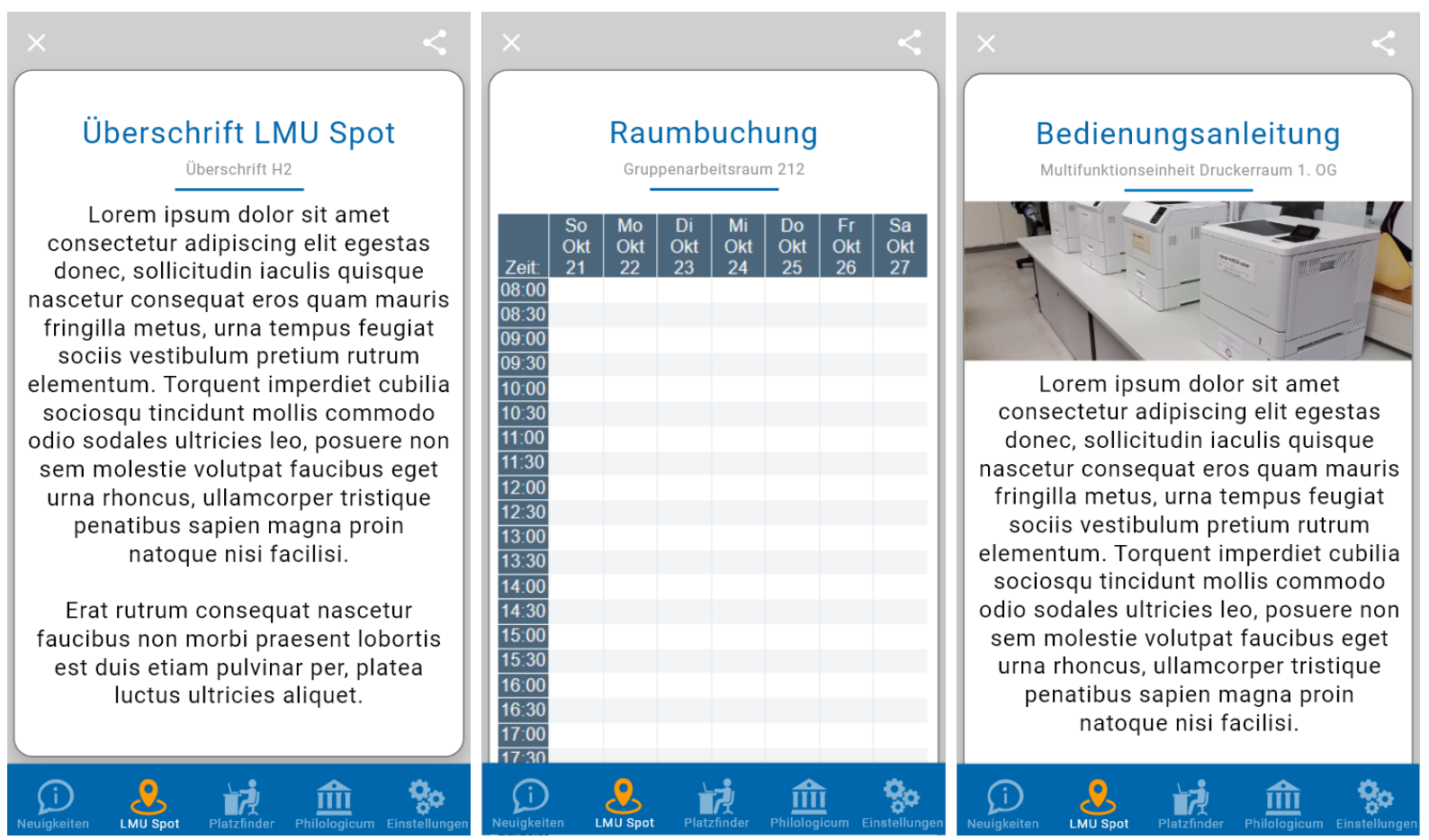

Abbildung 34: Detailansichten LMU Spot ${ }^{343}$

In Abbildung 34 sind die Detailansichten dargestellt. Wie auch die User Szenarien des Hauptbildschirmes, sind die Detailansichten flexibel und an weitere Use Cases anpassbar. Es ist ersichtlich, dass sich die Ansicht generisch in eine Überschrift, Zwischenüberschrift, Trennstrich und Inhalt, überwiegend in Form von Text unterteilt.

Detailansicht 1 zeigt hierbei die weiterführenden Informationen für die Zitate der Kunst am Bau. Detailansicht 2 präsentiert dagegen Visualisierung einer erfolgreichen Zuordnung des Standortes zu einem Gruppenarbeitsraum. Hierbei ist anzumerken, dass das bestehende Raumbuchungssystem, dessen Web-Frontend im responsive Design gestaltet ist, in die Anwendung eingebunden werden kann.

Abschließend ist exemplarisch die GUI für eine Bedienungsanleitung konzipiert worden. Der Nutzer befindet sich dazu in der Nähe eines technischen Gerätes und möchte weiterführende Informationen zu diesem angezeigt bekommen. Wichtig ist zu beachten, dass neben Text auch Bilder mit eingebunden werden müssen.

Hauptaufgabe des Systems ist die Bereitstellung von ortsabhängigen Informationen, da es sich bei dem Tab LMU Spot um diese handelt, sind alle drei Hauptkomponenten

${ }^{343}$ Quelle: Eigene Darstellung. 
des Systems involviert. Abbildung 35 analysiert den Workflow im Hinblick auf das Zusammenspiel der unterschiedlichen Elemente. Neben der Funktion der Anzeige soll in diesem Tab ebenfalls die Positionierung stattfinden.

In dem Testszenario in Kapitel 7.2.3 „Texterkennung - OCR“ wird beschrieben, dass die Anwendung eine Logik zur Standortermittlung implementiert bekommen wird, die eindeutige Kombinationen von Schlüsselwörtern oder Zahlen einem Standort zuordnet. Generell wäre es für den Nutzer vorteilhaft, wenn dieser nach Überschreitung einer bestimmten Zeit, ohne erfolgreiches Positioning von der Anwendung eine Hilfestellung bekommt. Ist als Ergebnis des Positionings eine Location-ID ermittelt worden, wird diese über eine API an der Middleware angefragt. Diese wiederum prüft intern ab, ob eine Verknüpfung für diese Location-ID vorhanden ist. Gemäß dem Fall, dass diese hinterlegt ist, antwortet die Middleware dem Frontend in Form einer Content-ID. Das Frontend fragt im nächsten Schritt die Content-ID beim Backend an. Im Folgenden prüft das Backend nun die Content-ID und liefert einen strukturierten Inhalt zurück. Das Frontend hat nun abschließend die Aufgabe den Inhalt anzuzeigen. Voraussetzung für die Kommunikation zwischen den Systemen ist das Bestehen einer Internetverbindung.

2) Request Location-ID

\section{Middleware 3) Resolve Location-ID}

4) Respond Content-ID

1) Positioning

\section{Frontend}

8) Display Content

5) Request Content-ID

\section{Backend}

6) Resolve Content-ID

7) Respond Content

Abbildung 35: Resolving-Workflow für den LBS $^{344}$

Je nach Grad der angestrebten Robustheit des Systems können hier im Vorgang der Auflösung der IDs, bei Middleware und Backend verschiedene Logiken zur Überprüfung implementiert werden. Grundsätzlich kann die Kommunikation zwischen den

${ }^{344}$ Quelle: Eigene Darstellung. 
einzelnen Komponenten zur Realisierung des LBS auf der demonstrierten Ebene abstrahiert worden.

Im Ergebnis der Evaluation wird darauf hingewiesen, dass die markierungsfreie Positionierungstechnik es dem Nutzer erschweren könnte, die Möglichkeit zur Interaktion zu erkennen. Es wird daher empfohlen, sofern möglich kleine visuelle Markierungen für ein optisches Feedback des Nutzers zu installieren. Dies gilt im Hinblick auf die Raumbuchungen und die technischen Geräte, nicht aber für die Kunst am Bau, da hier die visuelle Einheit nicht zerstört werden soll. Zur Wahrung der Einheitlichkeit wird empfohlen, das Icon, samt Beschriftung von „LMU Spot“, wie in der Bottom Navigation Bar dargestellt zu verwenden. Der Nutzer hat in Folge dessen einen visuellen Hinweis, dass an dieser Position eine Interaktion stattfinden kann.

\subsubsection{Frontend - Platzfinder}

Das System Platzfinder ist ebenfalls, wie in Kapitel 6.2.2 „Anforderungskatalog“ aufgeführt, eine extern einzubindende Plattform, dessen Web-Frontend im responsive Design gestaltet worden ist. ${ }^{345}$ Die Einbindung des Inhaltes kann direkt über das Backend stattfinden, ohne über die Middleware aufzulösen. Abbildung 36 zeigt eine mögliche Einbindung des Platzfinders in der GUI des Frontends. Die gelieferten Daten werden automatisiert über das externe System verarbeitet und müssen im Frontend lediglich zur Anzeige gebracht werden. Hierbei handelt es sich um einen weiteren ortsunabhängigen Service des Systems.

\subsubsection{Frontend - Philologicum}

Ergänzend zu den bisherigen ortsunabhängigen Services ist, gemäß den User Stories ein weiterer Bildschirm mit allgemeinen Informationen zum Philologicum entworfen worden. Dieser Tab kann alle sonstig relevanten Basisinformationen abdecken, die in keinem der anderen ortsunabhängigen Bildschirmen untergebracht werden können. Neben Informationen zu Öffnungszeiten, Kontaktdaten und der Verkehrsanbindung,

\footnotetext{
${ }^{345}$ Ein Beispiel für eine Raumreservierung ist unter https://raum.ub.uni-muenchen.de/week.php?area=7 zu finden.
} 
können gleichfalls Lagepläne oder die exemplarische Integration von Google-Maps in diesem Tab realisiert werden. Abbildung 36 stellt diesen Tab in der UI grafisch dar und kann als Entwurfsmuster betrachtet werden.
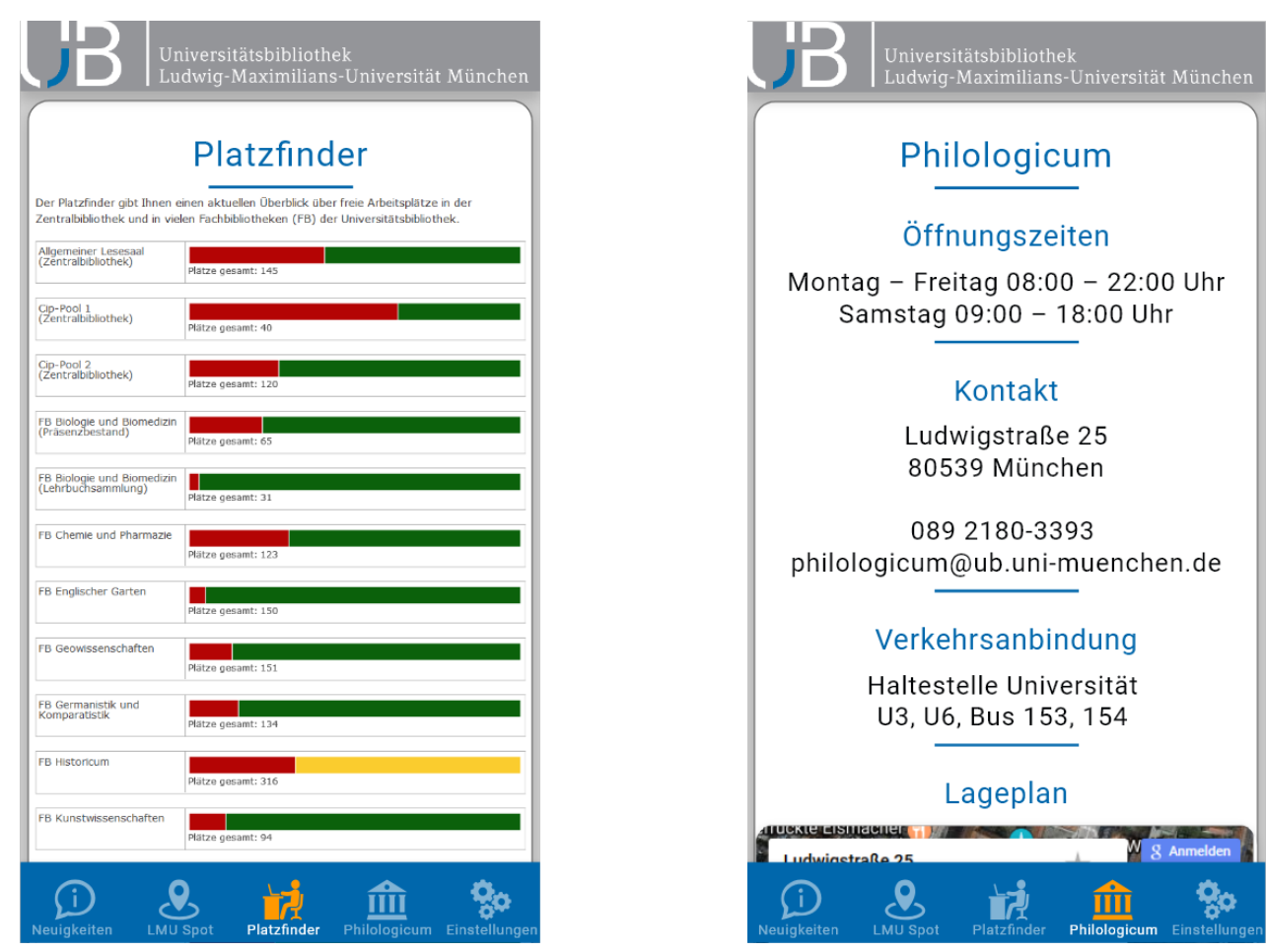

Abbildung 36: Sitzplatzkapazitäten mit dem System Platzfinder \& Basisinformationen zum Philologicum $^{346}$

\subsubsection{Frontend - Einstellungen}

Aufgrund diverser nichtfunktionaler Anforderungen an das Frontend ist ein zusammenfassender Tab, mit der Möglichkeit zur Anpassung der GUI an den Nutzer konzipiert worden. Da die Anwendung ortsunabhängige Informationen in Form von Neuigkeiten zum Philologicum bereithalten wird, bietet es sich an den Nutzer mittels Notifications darauf aufmerksam zu machen. Das Frontend sollte sich deshalb soweit konfigurieren lassen, dass diese Benachrichtigungen deaktiviert werden können.

In Abbildung 37 sind unter anderem zwei Optionen zur Verbesserung der Barrierefreiheit der GUI dargestellt. Neben der Option die gesamte Oberfläche für einen maximalen Kontrast in Schwarz-Weiß darzustellen, sollte die Schriftgröße variiert werden

\footnotetext{
${ }^{346}$ Quelle: Eigene Darstellung.
} 
können. ${ }^{347}$ Die Schrift für bedeutungstragenden Inhalt sollte zu keiner Zeit eine Größe von 14 Pixeln unterschreiten. ${ }^{348} \mathrm{Zu}$ beachten ist im Hinblick auf eine produktive Umsetzung, dass eventuell enthaltene Bilder ebenfalls in Schwarz-Weiß bereitgehalten oder entsprechende programmatische Möglichkeiten zur Konvertierung von Bildern innerhalb der mobilen OS geprüft werden.

Neben diesen Anpassungen hat der Nutzer darüber hinaus die Option, die GUI komplett in englischer Sprache anzeigen zu lassen. In der Konzeption des UI sind in den Einstellungen darüber hinaus Hinweise zu Impressum und Datenschutz der mobilen Anwendung untergebracht. Jedoch sei darauf hingewiesen, dass diese nur exemplarisch hinterlegt sind und im Produktivbetrieb noch der gültigen Rechtsgrundlage angepasst werden müssen.
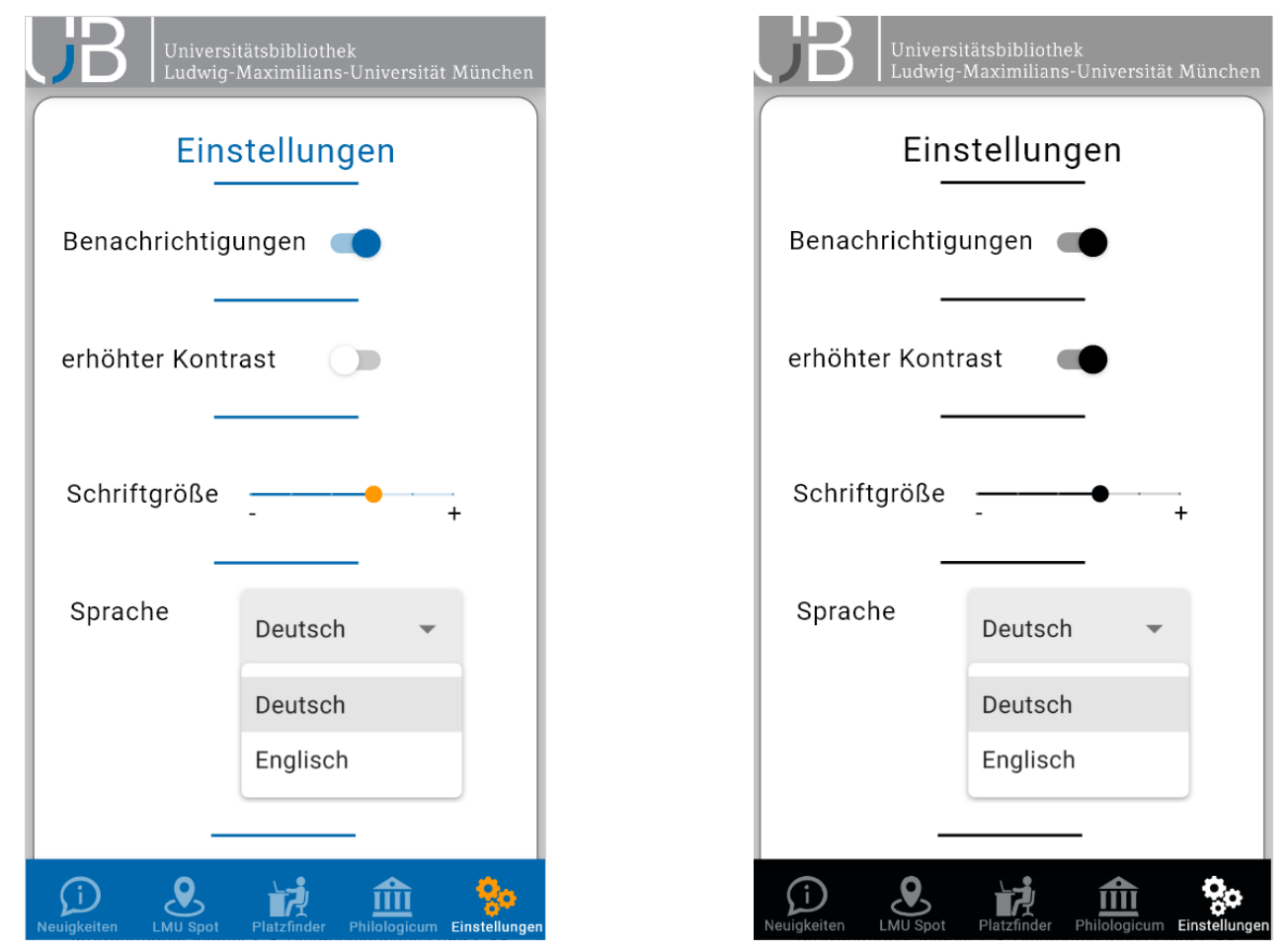

Abbildung 37: Einstellungen in normaler Ansicht und in maximalen Kontrast ${ }^{349}$

\footnotetext{
${ }^{347}$ Weiterführende Informationen werden vom W3C in Form der Web Content Accessibility Guidelines 2.0 angeboten und können unter: https://www.w3.org/TR/2008/REC-WCAG20-20081211/ abgerufen werden.

${ }^{348} \mathrm{Vgl}$. Google LLC $2018 \mathrm{i}$.

${ }^{349}$ Quelle: Eigene Darstellung.
} 


\subsubsection{Frontend - Share und Notifications}

Die in Abbildung 38 gezeigten zusätzlichen Bildschirme visualisieren die Möglichkeit des Nutzers, Inhalte zu teilen und die Benachrichtigungen außerhalb der Anwendung, über die Notification-Bar. Hierbei handelt es sich bei deren Design um Standardkomponenten des Android OS. Eine individuelle Anpassung ist möglich, jedoch nicht weiter erforderlich.

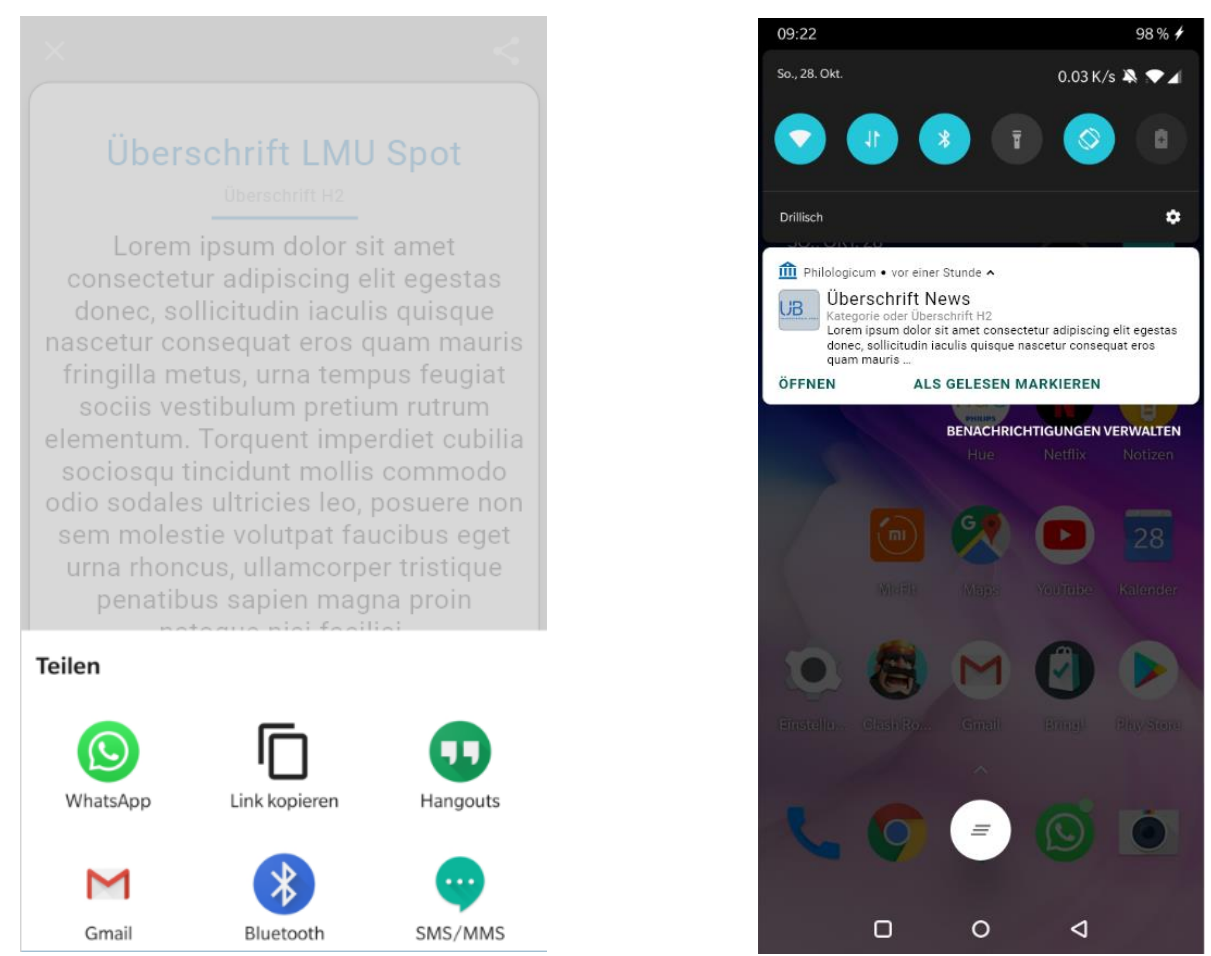

Abbildung 38: Bildschirme für das Teilen von Inhalten und Notifications ${ }^{350}$

\subsection{Konzeption der Middleware}

Die Middleware übernimmt in der Architektur des Systems überwiegend eine verknüpfende Aufgabe zwischen Front- und Backend. Sie soll als einfach zu bedienendes, webbasiertes Tool für die Mitarbeiter der UB entworfen werden. Je nach zukünftiger Verwendung des Systems kann die Middleware funktional flexibel erweitert werden und mehr als nur reines Verbindungselement sein. Das Design spielt eine untergeordnete Rolle und soll sich an der zu Grunde liegenden Funktionalität orientieren.

Wie im Anforderungskatalog benannt, handelt es sich überwiegend um die Verwaltung, genauer gesagt dem Hinzufügen und Löschen, der Verknüpfungen zwischen

\footnotetext{
${ }^{350}$ Quelle: Eigene Darstellung.
} 
Standort (Location) und Inhalt (Content). Darüber hinaus ist es für die Anwender sinnvoll bestehende Verbindungen abzufragen. Eine weitere Funktion, die in der Form nicht in den Anforderungen benannt ist, sondern sich erst im Zuge der Festlegung der zu verwendenden Positionierungstechnologie ergeben hat, ist die Administration der Schlüsselwörter (Keywords) bezüglich der Zuordnung zu einem Standort.

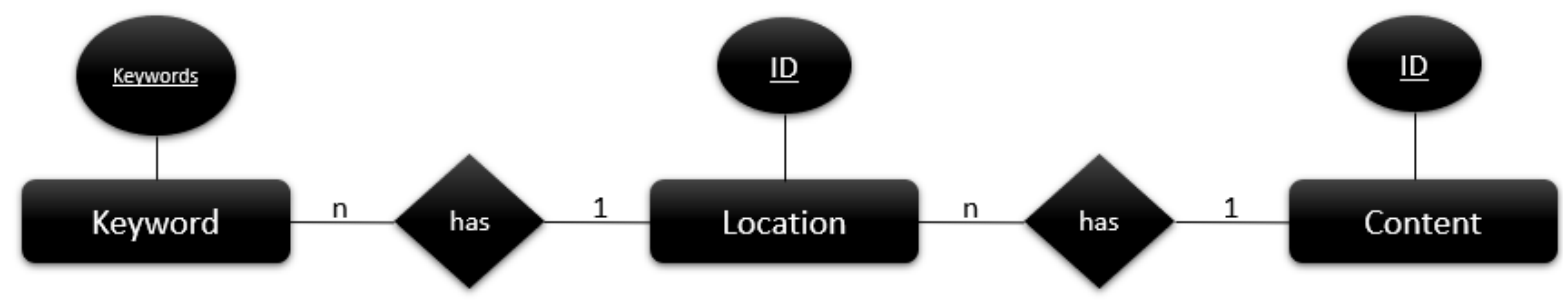

Abbildung 39: Vereinfachtes Entity-Relation-Ship-Modell der Verknüpfungen ${ }^{351}$

Die Beziehung der drei Entitäten zueinander, die in der Middleware verwaltet werden ist in Abbildung 39 dargestellt. Demnach können mehrere Keywords einer Location zugeordnet werden, aber die Keywords zeigen im Umkehrschluss immer genau auf eine Location. Damit kann eine Kombination aus Keywords immer nur einem Standort zugeordnet werden. Ähnlich verhält sich die Beziehung zwischen Location und Content. Mehrere Standorte können auf den gleichen Inhalt zeigen. Jedoch kann eine Location immer nur auf einen Content verweisen und nicht auf mehrere.

Im Rahmen der Konzeption ist bewusst auf eine vollständige Attributierung aller Entitäten verzichtet worden. Es bietet sich an für Location und Content jeweils IDs zu vergeben. Die Keywords hingegen sind für sich bereits eindeutige Zeichenketten, bestehend aus Buchstaben, Zahlen und Sonderzeichen. Diese können als Primärschlüssel verwendet werden und es sind demnach keine zusätzlichen IDs notwendig. Mit diesen Bedingungen werden die Integrität und Eindeutigkeit der Zuordnungen gewahrt und müssen dementsprechend in der Anwendung implementiert werden. Dies kann beispielsweise in Form von Plausibilitätsprüfungen stattfinden.

Durch die Separierung in einzelne Bausteine und Zuordnung einer festen Beziehung zueinander, können die einzelnen Entitäten in Zukunft mit verschiedenen Attributen versehen werden. Das System ist infolgedessen von Anfang an flexibel erweiterbar. Für die Location ist, die in Abbildung 40 gezeigte Attributierung denkbar. Dieser Ausbau, in der Beschreibung der Entitäten, könnte in Zukunft an verschiedenen Stellen

${ }^{351}$ Quelle: Eigene Darstellung. 
für weiterführende Informationen nachgenutzt werden. Als Konsequenz wäre es möglich in der Middleware zum Beispiel weitere Standorte zu verwalten.

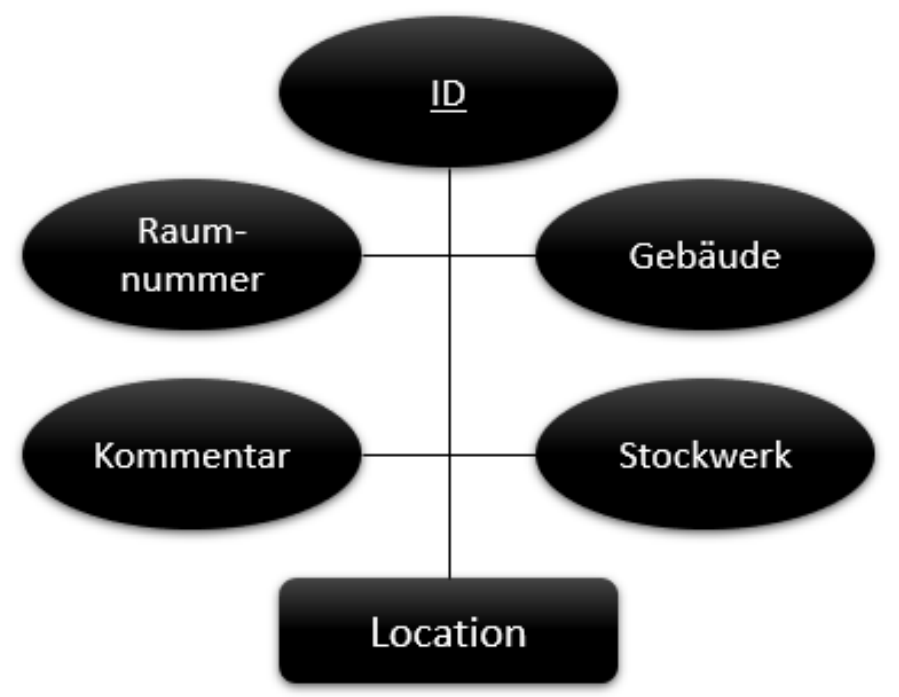

Abbildung 40: Mögliche Attribute der Entität Location ${ }^{352}$

Es ist denkbar, dass neben einer reinen Verwaltung der Verknüpfungen, die in Abbildung 39 dargestellten Entitäten selbst, in der Middleware verwaltet werden könnten. Auf eine umfangreiche Konzeption der einzelnen Arbeitsprozesse und deren Ausgestaltung wird jedoch im Folgenden verzichtet. Um den Aufwand der Umsetzung und die Komplexität der Middleware möglichst gering zu halten, wird empfohlen, zunächst lediglich die Hauptaufgabe des verknüpfenden Resolving-Mechanismus zwischen den Komponenten zu realisieren.

Zur Wahrung der Flexibilität des Systems ist es notwendig, die Kombinationen der Schlüsselwörter und Zuordnung zu einer Location variabel zu gestalten. Ein starres Konstrukt aus initialen Zuordnungen würde im Lauf der Zeit überholt werden und im Worstcase, bei jeder Änderung der Verknüpfungen eine Aktualisierung der mobilen Anwendung, in den jeweiligen Distributionskanälen nach sich ziehen.

Dahingehend ist es sinnvoller diese Zuweisungen ebenfalls über die Middleware zu verwalten. Es ist davon auszugehen, dass nach dem initialen Erfassen aller Verknüpfungen in unregelmäßigen Abständen Änderungen an der Datenbasis vorgenommen werden müssen. Es kann ein simpler Prüfmechanismus beim Initialisieren des Frontends genutzt werden, um zu kontrollieren, ob die verwendeten Kombinationen noch gültig sind. Abbildung 41 zeigt einen exemplarischen Workflow zur Realisierung des Mechanismus.

\footnotetext{
${ }^{352}$ Quelle: Eigene Darstellung.
} 
1) Initializing App

\section{Frontend}

4) Compare Versions
2) Request Version Keyword-Location

3) Respond Version Keyword-Location

\section{Middleware}

Abbildung 41: Workflow Versioncheck ${ }^{353}$

Der Mitarbeiter verwaltet die Verknüpfungen über die Middleware. Sobald die Aktualisierung abgeschlossen ist, generiert er über das Tool eine neue Versionsnummer. Das Frontend fragt die Versionsnummer bei Start der Anwendung ab und vergleicht diese mit der bisherig verwendeten. Sollten die Nummern nicht übereinstimmen, dann lädt das Frontend über die API alle Verknüpfungen, bestehend aus Zuordnung von Schlüsselwörtern zu Standorten, von der Middleware nach und aktualisiert seine interne Datenbasis. Stimmen die Versionen überein muss kein Aktualisierungsprozess stattfinden. Mit diesem Workflow kann neben der Flexibilität die zukünftige Erweiterbarkeit des Systems sichergestellt werden.

Die Eingaben der Anwender sollten an jeder Stelle der Middleware client- und serverseitig validiert werden. Dies erhöht nicht nur die Sicherheit des Systems, sondern ermöglicht ebenfalls eine Konsistenz der gespeicherten Daten. Im Hinblick auf die Verwaltung der Verknüpfungen zwischen Location-ID und Content-ID kann beispielsweise eine prüfende Logik implementiert werden, die nur eine bestimmte Anzahl aufeinanderfolgender Zahlen zulässt.

Bezüglich der Administration von Keywords und Location-IDs ist eine reine Beschränkung auf Zahlen nicht sinnvoll, sondern muss, zumindest bei den Schlüsselwörtern offen gestaltet werden, damit verschiedenste Buchstaben-, Zahlen und Sonderzeichenkombinationen gestattet sind. Es sollten jedoch geeignete Schutzmechanismen gegen gängige Angriffsvektoren, wie zum Beispiel »Structured Query Language (SQL)-Injections«, ergriffen werden, um zumindest ein gewisses Minimum an Sicherheit zu erfüllen.

\footnotetext{
${ }^{353}$ Quelle: Eigene Darstellung.
} 


\section{Prototypische Umsetzung „LMU Spot“}

Im letzten Kapitel der vorliegenden Arbeit sollen Teile der Konzeption prototypisch umgesetzt werden. Aufgrund der gesteckten Rahmenbedingungen, konkret der Priorisierung der standortabhängigen Workflows und den Umfang des Projektes ist eine vollständige Umsetzung im Rahmen der Masterarbeit nicht möglich. Vielmehr soll der Grundstein für die zukünftige Realisierung in der Praxis gelegt werden. Diese Basis soll zudem die Machbarkeit eines solchen Systems beweisen und führt zur Erreichung eines der Ziele dieser Untersuchung.

Die folgenden Kapitel zeigen den aktuellen Stand der prototypischen Systemkomponenten auf und weisen auf zukünftige Entwicklungs- und Erweiterungsmöglichkeiten hin. Es ist anzumerken, dass der, in der Implementierungsphase des Projektes entstandene, Quellcode ebenfalls Teil dieses Kapitels ist. Die entwickelte Logik wird in diesem Kapitel nur in Teilen aufgegriffen und sollte am besten direkt in der Kommentierung der jeweiligen Dateien nachgelesen werden. In dieser ist des Weiteren versucht worden mit „ToDo“-Hinweisen die entsprechenden Teile des Quellcodes zu kennzeichnen, bei dem für einen späteren Produktivbetrieb noch zusätzliche Logik implementiert werden sollte.

\subsection{Prototypische Umsetzung der Middleware - „LMU Linker“}

Im Fokus der Implementierung ${ }^{354}$ stand im Hinblick auf die Middleware die Umsetzung der Basisfunktionen im Vordergrund. Konkret sind die Funktionen „Anzeigen“, „Löschen“ und „Verknüpfen“ programmatisch realisiert worden. Abgerundet worden ist die Middleware durch die Entwicklung einer API zur Abfrage bestehender Verknüpfungen. Zum einen durch den Mitarbeiter selbst, zum Testen des Systems und zum anderen durch das Frontend, um den konzeptionierten Resolvingprozess zu realisieren.

Im Rahmen der Masterarbeit konnten die Workflows und Funktionen bezüglich der Keywords, aufgrund zeitlicher Restriktionen nicht praktisch entwickelt werden. Diese

\footnotetext{
${ }^{354}$ Die aktuelle Implementierung kann unter https://Imulinker.kai-barth.de/linker.html abgerufen werden. Es ist zu beachten, dass zum Zeitpunkt des Abrufs bereits Veränderungen am Quellcode vorgenommen worden sind, die zum Zeitpunkt der Erstellung der Masterarbeit noch nicht implementiert gewesen sind.
} 
Komponenten sind momentan lediglich als Platzhalter in der GUI hinterlegt und müssen bei der zukünftigen Umsetzung mit Logik befüllt werden. Darüber hinaus sind optionale Funktionen, wie das in Kapitel 8 „Konzeption des Systems“ beschriebene Festschreiben der POIs auf einzelne Standorte, beispielsweise mithilfe einer Karte nicht prototypisch realisiert worden. Diese Ergänzung würde jedoch für den produktiven Betrieb äußerst sinnvoll sein, damit die Mitarbeiter keine sekundären Informationsquellen für die möglichen Standorte im Philologicum nutzen müssen. Der Quellcode der Anwendung zum Zeitpunkt der Bearbeitung der vorliegenden Arbeit findet sich vollständig im Anhang ${ }^{355}$ und auf Github ${ }^{356}$.

Des Weiteren sind Videos zur Demonstration der Funktionsweise von LMU Linker im Anhang zu finden. ${ }^{357}$ Diese zeigen die prototypische Umsetzung der bisherigen Hauptfunktionen. Dies sind unter anderem das Verknüpfen einer Standort-ID mit einer Content-ID, sowie die Anzeige bereits hinterlegter Verbindungen und das Löschen bestehenden Verknüpfungen. Daneben wird die Verwendung der API von LMU Linker veranschaulicht.

\subsubsection{GUI der Webanwendung mittels Bootstrap}

Die Basis für die Webanwendung „LMU Linker" bilden HTML-Dateien zur Erstellung des Grundgerüstes. Auf die umfangreiche Entwicklung eigener Cascading Stylesheet (CSS)-Dateien ist zugunsten des Frameworkes Bootstrap ${ }^{358}$ verzichtet worden. Bootstrap ist ein freies Frontend-CSS-Framework, das eine weite Verbreitung aufweisen kann. ${ }^{359}$ Der Vorteil des Frameworkes ist, dass es eine ganze Reihe an typografischen Elementen, Formulare, Buttons und so weiter für die Gestaltung einer Website mitbringt. Darüber hinaus hält die Website von Bootstrap eine umfangreiche Dokumentation zur Verwendung der jeweiligen Elemente bereit. ${ }^{360}$ In Bezug auf unterschiedliche Anzeigeformate bietet Bootstrap eine Vielzahl an unterschiedlichen Viewports an.

\footnotetext{
${ }^{355}$ Pfad: ../Anhang/LMU Linker/Code

${ }^{356} \mathrm{https}: / /$ github.com/KaiBarth/Masterarbeit

${ }^{357}$ Pfad: ../Anhang/LMU Linker/[Dateiname].mp4

${ }^{358}$ Weiterführende Informationen unter: https://getbootstrap.com/

${ }^{359} \mathrm{Vgl}$. Mees 2015.

${ }^{360} \mathrm{Vgl}$. Introduction.
} 
Das Framework ist in der Version 4.0.0 verwendet worden. Die Einbindung in das Projekt erfolgte über einen Link in den jeweiligen HTML-Dateien. Es ist dabei immer sowohl das jeweilige JavaScript-, als auch CSS-Derivat verwendet worden, da sonst einige Animationen nicht korrekt angezeigt werden.

Im Wesentlichen sind die Elemente Navbar ${ }^{361}$, Jumbotron ${ }^{362}$, Forms ${ }^{363}$, Buttons ${ }^{364}$ und Alerts $^{365}$ verwendet worden. Das Element Jumbotron ist als eine Art einheitlicher Header eingesetzt worden, der neben der Navbar auf allen Seiten der Webanwendung präsent ist. Die Alerts hingegen sind für die Anzeige der Resultate der Datenbankabfragen genutzt worden. Je nach Ergebnis weisen diese eine andere Formatierung auf und geben dem Nutzer ein direktes Feedback zu seiner Interaktion. Das Farbschema ist in seiner Standardform in das Projekt integriert worden.

An dieser Stelle könnten zukünftig noch Anpassungen an das CD der Auftraggeberin vorgenommen werden. Im Hinblick auf die Möglichkeiten von Bootstrap lässt sich sagen, dass bisher nur an der Oberfläche gekratzt worden ist. Das Framework bietet noch zahlreiche weitere Komponenten und Funktionen, die sich zukünftig nachnutzen lassen könnten. Bootstrap ist als Framework auf das Prinzip „mobile-first“ ausgelegt und folglich nur sekundär für Desktop-Frontends ausgelegt. ${ }^{366}$ Diese Tatsache spiegelt sich auch in der aktuellen Oberfläche wieder und führt unter anderem dazu, dass beispielsweise die Eingabefelder in den Formularen über die gesamte Bildschirmbreite gestreckt werden. Hier sollten rasch Anpassungen für die Arbeit am Desktop vorgenommen werden, da die Mitarbeiter der UB in der Regel nicht mit mobilen Endgeräten die Middleware bedienen werden.

\subsubsection{Client- und serverseitige Logik mittels jQuery und PHP}

Die clientseitige Logik, um beispielsweise Nutzereingaben zu validieren oder Eingaben der Nutzer zu verarbeiten ist mithilfe von jQuery umgesetzt worden. jQuery ist eine freie JavaScript-Bibliothek, die Funktionen zur Navigation und Manipulation von

\footnotetext{
${ }^{361}$ Dokumentation unter: https://getbootstrap.com/docs/4.0/components/navbar/

${ }^{362}$ Dokumentation unter: https://getbootstrap.com/docs/4.0/components/jumbotron/

${ }^{363}$ Dokumentation unter: https://getbootstrap.com/docs/4.0/components/forms/

${ }^{364}$ Dokumentation unter: https://getbootstrap.com/docs/4.0/components/buttons/

${ }^{365}$ Dokumentation unter: https://getbootstrap.com/docs/4.0/components/alerts/

${ }^{366}$ Vgl. Bootstrap.
} 
HTML-Dokumenten bereithält sowie die asynchrone Kommunikation via Asynchronous JavaScript and XML (»AJAX«) erheblich erleichtert. ${ }^{367}$ Der Prototyp von LMU Linker enthält bereits umfangreichen Code zur Validierung der Nutzereingaben im Hinblick auf die angebotenen Formularfelder. Diese clientseitigen Prüfroutinen können für den produktiven Betrieb genutzt werden und bedürfen keiner weiteren Anpassungen. Jede HTML-Seite die Nutzereingaben verarbeitet hat ihre eigene jQuery-Datei. In der Regel findet neben der Validierung und Steuerung des Nutzerfeedbacks über verschieden farbige Hinweise mittels Alerts, auch die Verarbeitung des Inputs in Form der Weitergabe an die serverseitigen PHP: Hypertext Preprocessor (PHP)-Skripte statt. Des Weiteren haben die jQuery-Skripte die Aufgabe die Antwort des Servers via »AJAX« auszuwerten und dem Nutzer ein visuelles Feedback zu seiner Interaktion zu liefern. Die Kommunikation zwischen Client und Server findet, wie die Kommunikation zwischen Frontend, Middleware und Backend über das Datenformat »JSON« statt.

Der Quellcode kann dahingehend optimiert werden, dass die Anzahl der eingebundenen JavaScript-Dateien reduziert und redundanter Code in einer gemeinsamen Datei zur Verfügung gestellt wird. Eventuell kann darüber hinaus der existierende Quellcode in einer Datei gebündelt werden.

Neben der clientseitigen Validierung der Nutzereingaben ist ein serverseitiges Pendant in PHP implementiert worden, dass kaskadierend alle übermittelten Werte validiert und auf Plausibilität überprüft. Sollte ein Fehlerfall auftreten, übermitteln die Skripte ein Feedback via »JSON« zurück. Im Erfolgsfall übernehmen diese hingegen die jeweiligen Operationen auf der Datenbank und wickeln alle Transaktionen serverseitig ab. In der derzeitigen Version der Routinen ist vor allem Wert darauf gelegt worden, möglichst umfangreiche Filtermechanismen einzubauen, die Angriffsvektoren auf das System, wie beispielsweise "SQL-Injections« minimieren.

Der Quellcode ist bereits in der Lage Verknüpfungen zwischen Location-IDs und Content-IDs herzustellen, anzuzeigen und zu löschen. Die PHP-Skripte enthalten somit die Logik zur Wahrung der Integritäts- und Plausibilitätsregeln aller zu verarbeitenden Daten, wie in Kapitel 8.2 „Konzeption Middleware“ beschrieben.

Teile des Quellcodes sind bereits modularisiert worden, sodass diese in verschiedenen Skripten eingebunden werden können, um redundanten Code zu vermeiden.

\footnotetext{
${ }^{367}$ Vgl. jQuery Foundation 2018.
} 
Jedoch ist an dieser Stelle Potential zur Optimierung im Hinblick auf die einzelnen Validierungsschritte gegeben. Wie bei den jQuery-Dateien können die PHP-Skripte noch mehr vereinheitlicht werden, um die Weiterentwicklung, Wartung und Anpassbarkeit zukunftsfähiger zu gestalten.

Darüber hinaus können die derzeit angebotenen Methoden zur Verwaltung der Verknüpfungen vor allem in Bezug auf die Usability verbessert werden. Momentan ist beispielsweise nur die Verarbeitung einzelner Verknüpfungen möglich. Denkbar wäre es jedoch für den Produktivbetrieb Routinen zu ergänzen, die auch eine Massenverarbeitung möglich machen. Beispielsweise alle Verknüpfungen zu einer bestimmten Content-ID zu löschen, statt dies in einzelnen Arbeitsschritten durchzuführen. Hierbei wird die künftige praktische Handhabung einzelner Workflows maßgeblich für die Weiterentwicklung des Systems sein.

\subsubsection{Persistente Datensicherung mittels InnoDB}

Zur persistenten Sicherung der Nutzereingaben ist eine »InnoDB«-Datenbank für das Datenbankmanagementsystem MySQL verwendet worden. Es ist jedoch ebenfalls problemlos möglich ein anderes Speichersubsystem, zur Sicherung der Daten zu nutzen. Wichtig ist lediglich zu beachten, dass es sich um ein MySQL-Datenbankverwaltungssystem handeln sollte, da sonst Anpassungen an den PHP-Skripten notwendig sind.

Zur Umsetzung der benannten Hauptfunktionen inklusive der API zu LMU Linker wird derzeit die Datenbank „Imulinker_DB“ ${ }^{\text {“368 }}$ unter Verwendung der Tabelle „Iocation_content_link" mit folgenden Datenbankschema verwendet:

\begin{tabular}{|l|l|c|c|c|c|}
\hline \multicolumn{9}{|c|}{ Datenbankschema } \\
\hline 1 & Name & Primary Key & Typ & Null & Auto Increment \\
\hline 2 & location_id & ja & int(10) & Not Null & ja \\
\hline 3 & content_id & nein & $\operatorname{int}(10)$ & Not Null & nein \\
\hline
\end{tabular}

Tabelle 13: Datenbankschema LMU Linker ${ }^{369}$

\footnotetext{
${ }^{368}$ Ein Export der Datenbank in Form von SQL ist dem Anhang zu entnehmen.

Pfad: ../Anhang/LMU Linker/Datenbank/Imulinker_db.sql

${ }^{369}$ Quelle: Eigene Darstellung.
} 
Die Datenhaltung ist bezüglich des Prototyps vergleichsweise simpel, kann jedoch im Verlaufe des Projektes einfach und schnell erweitert werden. Die gewählte OpenSource-Basis MySQL wird zu keiner Limitierung der Möglichkeiten führen. Mit zunehmendem Umfang der Datenbank ist in Betracht zu ziehen, die aufgestellten Integritätsregeln in den serverseitigen PHP-Skripten entsprechend zu erweitern, um eine konsistente Datenbasis zu gewährleisten.

\subsubsection{Bereitstellung der Daten mittels einer API}

Wie in der Konzeptionierung beschrieben kommunizieren die einzelnen Systemkomponenten über Schnittstellen miteinander. Die Middleware muss demnach eine API vorhalten, damit das Frontend die Verknüpfungen zwischen Standort und Inhalt abfragen kann. Im Zuge der prototypischen Implementierung ist es als nützlich erachtet worden, dass der Mitarbeiter zukünftig die Schnittstelle über ein geeignetes Webformular abfragen kann, um die korrekte Funktionsweise zu überprüfen.

Als strukturiertes Datenformat ist »JSON«, aufgrund seiner Verbreitung gerade bei Webanwendungen ausgewählt worden. Prinzipiell würde die API selbstverständlich in gleicher Weise mit anderen strukturierten Datenformaten, wie XML funktionstüchtig sein. Hierzu sind kleinere Justierungen an den client- und serverseitigen Skripten notwendig. Wie bei einer API für Webservices üblich, wird diese über die URL angesprochen. Neben dem Basis Teil der URL besteht ein Request im Moment aus drei weiteren obligatorischen Parametern. So könnte die URL zur Anfrage einer Verknüpfung exemplarisch wie folgt lauten:

„https://Imulinker.kai-barth.de/php/api.php?format=json\&id_type=location_id\&id=1“"

Der Parameter „format“ definiert das Datenformat der Antwort des Servers. Dies ist im Rahmen der prototypischen Umsetzung immer »JSON«. Der zweite Parameter „id_type“ legt fest, welcher Typ an der Datenbank angefragt werden soll. Zukünftig wäre es denkbar Verknüpfungen über Content-IDs abzufragen, oder andere zukünftige Identifikatoren. Der letzte Parameter „id“ schließlich ist die konkrete ID, die abgefragt werden soll. Aufgrund der bereits hinterlegten Parameter, ist eine weitestgehende Flexibilität in der API implementiert worden, die es zukünftig einfacher gestaltet das System anzupassen und zu erweitern. 
Die Schnittstelle liefert als Ergebnis der Abfrage ein Ergebnis im »JSON«-Format zurück. Dieses kann zum Beispiel wie folgt aussehen:

\{"type":"success","requested_id_type":"location_id", "requested_id":"1", "linked_id_type":"content_id","sum":1,"linked_id":"1"\}

Dieser Response würde im Erfolgsfall zurückgegeben werden. Neben der Auflistung der angefragten Parameter wird ausgeliefert, was abgefragt worden ist und wie die verknüpfte ID ist. In diesem Beispiel ist mit Location-ID 1 die Content-ID 1 verknüpft. Sollte keine Verknüpfung vorhanden sein, dann würde der Server folgendes antworten:

\{"type":"norows","text":"Zu dieser ID sind keine Eintraege vorhanden."

Neben dem Typus „success“ und „,norows“ findet derzeit noch der Fehlerstatus „error" Anwendung. Dieser wird jedoch nur verwendet, wenn die Validierung der übermittelten Parameter fehlschlägt oder diese leer sind. Die API könnte in Zukunft dahingehend ausgebaut werden, dass nicht nur Zustände abgefragt werden, sondern zeitgleich Änderungen im System gemacht werden können, direkt über die Schnittstelle ohne eine separate Erfassung über das Webinterface von LMU Linker.

\subsection{Prototypische Umsetzung des Frontends - „Philologicum“}

Das Fundament für die prototypische Entwicklung des Frontends in Form der mobilen Anwendung „Philologicum“ bilden die bisher aggregierten Informationen. Vor allem im Hinblick auf die Evaluation der Methoden zur Standortbestimmung, der Konzeptionierung einzelner Workflows sowie der graphischen Oberfläche der App. Im Rahmen des Versuches zur Umsetzung der Machbarkeit eines Frontends, sind all diese Informationen gebündelt in die Realisierung eines ersten Prototypens geflossen. Die folgenden Abschnitte repräsentieren den aktuellen Stand der Anwendung zum Zeitpunkt der Masterarbeit. Neben kurzen Erklärungen zur Herangehensweise und Lösung verschiedener Probleme soll ein Ausblick auf zukünftige Weiterentwicklungsmöglichkeiten der App gegeben werden.

Im Hinblick auf die evaluierten Methoden zu Standortbestimmung ist bereits an dieser Stelle positiv zu erwähnen, dass der Prototyp der mobilen Anwendung lediglich zwei Berechtigungen benötigt, um vollständig funktionstüchtig zu sein. Diese in der „AndroidManifest"“-Datei verankerten Rechte sind der Zugriff auf die Kamera und auf das 
Internet. Es ist zu beachten, dass lediglich der Hardwarezugriff auf die Kamera einer Zustimmung des Nutzers bedarf. Die Freigabe des Internets ist Standard und wird automatisch von Android gewährt. ${ }^{370}$

Neben dem sparsamen Zugriff auf verschiedene Ressourcen des Smartphones ist abschließend zu nennen, dass bei allen verwendeten Entwicklungskomponenten auf die Abwärtskompatibilität zu Android 4.4, gemäß der Anforderung FE 0240, geachtet worden ist. Zur Sicherstellung der Integrität ist im Android Studio ${ }^{371} \mathrm{im}$ »Gradle«-Skript die „minSDKVersion“ auf das API-Level 19 fixiert worden. Android Studio prüft anschließend eigenständig, ob alle verwendeten Komponenten diese Anforderung erfüllen.

Ein kurzes Demonstrationsvideo, zum Stand der App während der Entwicklungsphase ist im Anhang zu finden. ${ }^{372}$ Unter anderem verdeutlicht diese Darstellung die Einbindung der Fremdsysteme Raumbuchung und Platzfinder. Interessant ist jedoch vor allem die Präsentation von LMU Spot, die anhand von 4 Beispielen demonstriert wird. Die ersten beiden Beispiele sind jeweils Zitate der Kunst am Bau, zu denen weiterführende Informationen eingeblendet werden. ${ }^{373}$ Der dritt eingescannte Text ist eine Raumnummer des Philologicums und zeigt die Einbindung des Raumbuchungssystems. ${ }^{374}$ Das letzte Beispiel schließlich ist stellvertretend für die Einbindung diverser Anleitungen, im Hinblick auf die Multifunktionseinheiten zu sehen. ${ }^{375}$

Der Quellcode der prototypischen Umsetzung ist dem Anhang zu entnehmen. ${ }^{376}$

\subsubsection{Prototypische Umsetzung der Standortbestimmung}

Kernelement der prototypischen App „Philologicum“ ist die Demonstration der Machbarkeit zur Standortbestimmung, Umsetzung des konzeptionierten Resolvingprozesses und damit verbunden die Anzeige weiterführender Informationen, basierend auf der jeweiligen Positionierung im Raum. Grundlage für die Positionsbestimmung ist

\footnotetext{
${ }^{370}$ Vgl. Künneth 2018, S. 298.

${ }^{371}$ Zur Entwicklung des Prototypens ist Android Studio in der Version 3.2.1 verwendet worden.

${ }^{372}$ Pfad: ../Anhang/Philologicum/Philologicum.mp4

${ }^{373}$ Tafel 1: Pfad: ../Anhang/Philologicum/Beispiele/Tafel 1.pdf

Tafel 2: Pfad: ../Anhang/Philologicum/Beispiele/Tafel 2.pdf

${ }^{374}$ Pfad: .../Anhang/Philologicum/Beispiele/Raum.png

${ }^{375}$ Pfad: ../Anhang/Philologicum/Beispiele/Mufu 1.docx

${ }^{376} \mathrm{Pfad}$ : ../Anhang/Philologicum/App/
} 
das Ergebnis der Evaluation aus Kapitel 7. Die bereits eruierte Methode der Texterkennung, unter Zuhilfenahme der Kamera des Smartphones, in Kombination mit der Android Bibliothek „Google Mobile Vision Text APl“ hat sich bei der prototypische Umsetzung bewährt. Der Nutzer scannt die Zitate der Kunst am Bau, Raumnummern oder auch die Namen der Mulitfunktionseinheiten in den Druckerräumen ein. Über einen Fingerprinting Algorithmus wird anschließend der zugeordnete Standort eruiert. Im Gegensatz zur experimentellen App, die im Rahmen der praktischen Tests in Kapitel 7.2.3 entwickelt worden ist, ist der Quellcode für den Prototypen weiter individualisiert worden. Das Rendering in der Kamera-Preview der Anwendung ist beispielsweise entfernt worden, da dieser Vorgang für den Nutzer nicht von Interesse ist. Im Fokus steht allein die rasche Erkennung des Standortes als Ergebnis, aber nicht der Prozess, der zum Ergebnis führt.

Darüber hinaus enthält die „MainActivity“ nun nicht mehr die Logik für die Zuordnung von Schlüsselwörtern zu den entsprechenden Standorten, sondern diese ist in eine separate Klasse „LocationMap“ ausgelagert worden. Diese Hilfsklasse nutzt eine HashMap $^{377}$, um die Verknüpfungen herzustellen. Dabei implementiert sie momentan eine Methode zum initialen Aufbau der Verbindungen beim Laden der Anwendung sowie eine Methode zur Überprüfung, ob der von der Kamera erkannte Text ein Schlüsselwort ist und welchem Standort dieses zugeordnet ist. Beide Methoden werden von der „MainActivity“ aufgerufen und verwendet. Zum jetzigen Zeitpunkt der Entwicklung ist die Umsetzung der Trainingsphase des Systems noch statisch. Alle exemplarischen Verquickungen sind hart codiert hinterlegt. Wie in der Konzeptionierung in Kapitel 8.2 beschrieben sollte für den Echtbetrieb eine geeignete Flexibilisierung integriert werden, sodass die Verbindungen dynamisch abgerufen werden können.

Ist eine erfolgreiche Standortbestimmung über die Methode „findLocationld“ der Klasse LocationMap durchgeführt worden, wird die ermittelte Location-ID an die „DetailsActivity“ weitergereicht. Die Abwicklung findet, wie in Android üblich über einen Intent ${ }^{378}$ statt. Im Zuge verschiedener Tests hat sich herausgestellt, dass das Testgerät, ein One Plus 6 die Logik des Quellcodes so rasch verarbeitet, dass eine Endlosschleife bei der Prozessierung entsteht. Die Kamera detektiert den Text so schnell,

\footnotetext{
${ }^{377}$ Weitere Informationen unter: https://developer.android.com/reference/java/util/HashMap

${ }^{378}$ Weitere Informationen unter: https://developer.android.com/reference/android/content/Intent
} 
dass die „DetailsActivity“ sehr oft hintereinander aufgerufen wird und der Nutzer immer wieder zurück zur „MainActivity“ navigieren muss. Um dies zu unterbinden ist eine »Flag« gesetzt worden, die eine wiederholte Verarbeitung durch die Anwendung sperrt und sicherstellt, dass der Standort nur einmalig bestimmt wird.

Die bereits mehrfach erwähnte Klasse „DetailsActivity“ übernimmt im Folgenden mehrere Aufgaben zur Realisierung des unter Kapitel 8.1.3 beschriebenen Workflows. Zum einen ermittelt sie die verknüpfte Content-ID über die Middleware „LMU Linker" und zum anderen fragt sie diese Content-ID dann bei dem Backend ab. Die Antwort des Backends stellt die „DetailsActivity“ abschließend noch grafisch dar. Zur Abfrage der Middleware wird die im Kapitel 9.1.4 beschriebene API genutzt. Die Abwicklung des Requests wird über die Programmbibliothek „Volley“379 realisiert. Diese Bibliothek bietet eine ganze Palette an Methoden zur Abfrage von Netzwerkverbindungen an. ${ }^{380}$ Es ist zu beachten, dass Volley in den „Dependencies“ von "Gradle“ untergebracht werden muss. ${ }^{381}$ Unter anderem können »JSON«-Objekte über einen Request abgefragt werden. Die App baut hierzu gemäß den API-Spezifikationen eine URL zusammen und fragt diese bei der Middleware ab. Als Ergebnis erhält die Anwendung ein »JSON«-Objekt, dass nach der Content-ID geparst werden kann.

Nach erfolgreichem Abschluss des Resolvingprozesses wird der Inhalt, der der Content-ID zugeordnet ist vom Backend geladen und mittels einer WebView ${ }^{382}$ angezeigt. Detaillierte Informationen zur Nutzung und Anzeige von Inhalten mittels WebView ist dem folgenden Kapitel 9.2.2 zu entnehmen. Es ist anzumerken, dass zum Zeitpunkt der Erstellung des Prototypens noch keine API zum Backend und ebenfalls noch keine konkreten Inhalte, die in der zukünftigen Anwendung zur Anzeige gebracht werden sollen, von Seiten der Auftraggeberin vorgelegen haben. Aus diesem Grund ist mit Platzhaltern zu Demonstrationszwecken gearbeitet worden. Der zu Grunde liegende Mechanismus ist jedoch flexibel erweiterbar und kann mit marginalen Anpassungen am Quellcode für den Echtbetrieb parametrisiert werden.

Auf die Implementierung von Error-Handlern ist an dieser Stelle, aufgrund des prototypischen Charakters verzichtet worden. Für den zukünftigen Betrieb ist jedoch geeigneter Code zu ergänzen, damit Fehlerfälle in korrekter Art und Weise abgefangen

\footnotetext{
${ }^{379}$ Weitere Informationen unter: https://developer.android.com/training/volley/

${ }^{380}$ Vgl. Google LLC 2018j.

${ }^{381}$ Vgl. ebd.

${ }^{382}$ Weitere Informationen unter: https://developer.android.com/reference/android/webkit/WebView
} 
werden können. Des Weiteren sollte generell eine Implementierung von Quellcode für den Fall vorgenommen werden, dass die mobile Anwendung an Knotenpunkten, an denen Inhalt nachgeladen bzw. Interaktion des Nutzers stattfinden wird, zu lange lädt. Beispielsweise kann dies eintreten, wenn ein bestimmter Zeitrahmen zur Erkennung von Schlüsselwörtern oder zum Laden von Inhalten benötigt wird. Ziel ist es dem Anwender ein entsprechendes Feedback zu geben. Dies trifft natürlich generell auch für den Fall zu, dass keine Internetverbindung besteht.

Für den produktiven Einsatz der Anwendung wird darüber hinaus empfohlen eine umfangreiche Trainingsphase des Systems, in Bezug auf die Erkennung der Schlüsselwörter stattfinden zu lassen. Wie in Kapitel 7.2.3 angedeutet sollte die App über eine intensive Vergleichsbasis für das Bestimmen des Standortes verfügen. Es könnten beispielsweise alle Zitate der Kunst am Bau mit verschiedenen Endgeräten eingelesen und über Microsoft Excel Übereinstimmungen in den gefundenen Texten gesucht werden. Diese initiale Trainingsphase kann mit der entwickelten Anwendung aus Kapitel 7.2.3 umgesetzt werden. Vorteil wäre, dass eine solide Ausgangsbasis für eine erfolgreiche Standortbestimmung gewährleistet werden kann. Darüber hinaus kann somit eventuell auch die Erkennung nicht lateinischer Schrift erfolgen und einen der wenigen Nachteile dieser Positionierungsmethodik abgedämpft werden.

\subsubsection{Prototypische Umsetzung der GUI}

Grundlage für die Entwicklung der GUI ist das in Kapitel 8.1 erarbeitete Konzept für die Oberflächengestaltung. Ein komplette 1:1 Adaption ist im Rahmen des Prototyps nicht möglich gewesen, jedoch sind alle elementaren Bestandteile wie die Bottom Navigation Bar bereits integriert. Die einzelnen Tabs der App sind mithilfe von Fragments $^{383}$ umgesetzt worden. Vorteilhaft ist in diesem Kontext, dass diese im Gegensatz zu gewöhnlichen Bedienelementen ebenfalls Logik implementieren können. ${ }^{384}$

Dadurch ist es möglich in der „MainActivity“ lediglich die Logik für die Standortbestimmung unter zu bringen und den Quellcode für die restlichen Tabs in einzelne

\footnotetext{
${ }^{383}$ Weitere Informationen unter: https://developer.android.com/guide/components/fragments

${ }^{384}$ Vgl. Künneth 2018, S. 147.
} 
Fragments zu separieren. Ein „setOnNavigationltemSelectedListener ${ }^{4385}$ auf der Bottom Navigation Bar sorgt nur noch für einen Austausch der Fragments, basierend auf der Auswahl des Nutzers. Die jeweiligen Fragments enthalten nun den Code für die jeweiligen Tabs und befüllen diese mit Inhalt.

Im aktuellen Stand der Entwicklung besteht die Oberfläche des Tabs LMU Spot im Wesentlichen aus der Vorschau des Kamerabildes und einer WebView zur Anzeige der abgerufenen Inhalte. Deswegen soll an dieser Stelle beispielhaft das „PlatzfinderFragment" zur Integration des externen Systems „Platzfinder" betrachtet werden. Innerhalb des Fragments wird der Inhalt, den dieses System liefert über eine WebView eingebunden. Erleichtert wird diese Möglichkeit durch die Tatsache, dass das System seine Daten mittels eines responsiven Layouts unter einer fixen URL zur Verfügung stellt. Ziel ist es somit die informationstragenden Inhalte des Systems in der App darzustellen. Wird die Website von Platzfinder nun einfach in einer WebView geladen, hat der Nutzer dieselben Interaktionsmöglichkeiten, wie bei einem normalen Aufruf der Website. Er kann unter anderem die Suche aufrufen oder innerhalb der WebView navigieren.

Um dies zu unterbinden, ist es möglich die geladene Website mittels JavaScript-Injections nachträglich zu manipulieren. Es wird zusätzlicher Quellcode in die geladene Website eingebunden und ausgeführt. Zur Realisierung des Ziels sind folglich zusätzliche JavaScript-Funktionen implementiert worden, um alle unerwünschten HTMLDOM-Elemente nicht zur Anzeige zu bringen. Nach erfolgreicher Implementierung des Codes ist jedoch aufgefallen, dass die Seite schon während des Ladevorganges in der Anwendung angezeigt wird und der Nutzer sehen kann, dass nachträglich Teile der Seite entfernt werden. Zur Beseitigung dieses Problems kosmetischer Natur ist der WebView, bis zum vollständig Abschluss aller JavaScript-Manipulationen ein Ladebalken vorgeschaltet. Dieser ist mithilfe des Widgets ProgressBar ${ }^{336}$ umgesetzt worden. Ein simples Ein- und Ausblenden des Widgets genügt, damit der Nutzer die Veränderung der Website nicht bemerkt.

Im Rahmen der Realisierung eines Prototypens ist die Anzeige von Inhalten mittels einer WebView ausreichend. Jedoch ist für den zukünftigen produktiven Betrieb zu

\footnotetext{
${ }^{385}$ Weitere Informationen unter: https://developer.android.com/reference/android/support/design/widget/BottomNavigationView.html

${ }_{386}$ Weitere Informationen unter: https://developer.android.com/reference/android/widget/ProgressBar
} 
prüfen, ob eine WebView als Mittel der Wahl zur Darstellung des Inhaltes immer noch zielführend ist. Problemtisch wird es, sofern der Inhalt in seiner Darstellung durch den Nutzer angepasst werden soll. Dies wäre zwar über die bereits erwähnten JavaScriptInjections möglich, jedoch obliegt der Grad der Anpassbarkeit den Möglichkeiten von JavaScript. Geschickter ist es den Inhalt, der vom Backend übermittelt wird, in einzelne Text- ${ }^{387}$ bzw. ImageViews ${ }^{388}$ auszulagern, da diese einfacher und tiefgreifender manipuliert werden können. Vor allem wird dies notwendig werden, wenn die Barrierefreiheit vollständig in der mobilen Anwendung umgesetzt werden soll, wie in den Anforderungen definiert.

Abschließend sei noch erwähnt, dass für die Programmierung der App auf gängige Verfahren, wie der Nutzung der Datei „strings.xml“ zur vereinfachten Anpassbar- und Wartbarkeit des Quellcodes, zurückgegriffen worden ist. Mit dieser Methode ist es prinzipiell möglich Texte an einem zentralen Ort zu speichern und erleichtert die zukünftige Hinterlegung von Übersetzungen in diverse Sprachen massiv. ${ }^{389}$ Gleiches gilt für weitere Ressourcen-Dateien, wie „colors.xml“ oder „styles.xml“ zur Anpassung des Erscheinungsbildes der Oberfläche.

\footnotetext{
${ }^{387}$ Weitere Informationen unter: https://developer.android.com/reference/android/widget/TextView ${ }^{388}$ Weitere Informationen unter: https://developer.android.com/reference/android/widget/ImageView ${ }^{389} \mathrm{Vgl}$. Künneth 2018, S. $115 f$
} 


\section{Resümee}

Zusammenfassend lässt sich sagen, dass mithilfe fundierter Analysen die Machbarkeit eines mit standortbasierten Informationen arbeitenden Systems demonstriert worden ist. Hilfreich ist neben der Untersuchung des aktuellen wissenschaftlichen Stands der Technik, gleichermaßen die Konkretisierung und Abstrahierung des geplanten Vorhabens sowie die umfassende praktische Evaluation gewesen. Die Aggregation aller Informationen hat wesentlich zur Synthese einer, in der Form im Bibliothekswesen bisher ungenutzten, Methodik zur Standortbestimmung beigetragen.

Es ist zu erwähnen, dass dieses Projekt nicht nur für die Auftraggeberin nützlich ist. Vielmehr können die gewonnen Erkenntnisse im Rahmen des bibliothekarischen Ökosystems adaptiert, parametrisiert und weiter ausgebaut werden. Einer Nachnutzung durch Dritte, die ein ähnliches Szenario in Planung haben, steht nichts im Weg. Natürlich müssen konkrete Ausgangsbedingungen eruiert und evaluiert werden, sodass die vorgestellte Methode zur Standortbestimmung nicht in allen Fällen das Mittel der Wahl sein kann. Jedoch ist die konzipierte Architektur des Systems flexibel genug, um gegebenenfalls mit anderen Verfahren zur Positionsbestimmung betrieben zu werden.

Für die UB der LMU ist in Ihrem Anwendungsszenario die optimale Methodik zur Positionsbestimmung gefunden worden. Die erfolgreiche Implementierung des Prototypen auf Basis von Middleware und Frontend bildet den Ausgangspunkt für eine Weiterentwicklung hin zur Nutzung im Echtbetrieb. Mit den gesammelten Forschungserkenntnissen kann erfolgreich das Fundament für einen LBS aufgebaut werden.

Alle Verantwortlichen und Projektteilnehmer müssen sich jedoch im Klaren darüber sein, dass die gemachten Schritte zunächst der Beginn eines langen Weges, hin zum Produktivbetrieb, sind. Auf diesem sind noch zahlreiche bisher unangetastete Hindernisse zu erklimmen, beispielsweise die Trainingsphase des Systems, die Anbindung des bestehenden Backends zur Verwaltung der Inhalte über eine separate API oder auch Planungsarbeiten, wie das Identifizieren konkreter Standorte. Diese Masterarbeit kann jedoch als Wegweiser für das zu realisierende Unterfangen dienen.

Bereits Franz Kafka sagte: „Wege entstehen dadurch, dass man sie geht." - diesen Leitsatz sollte sich die UB für die Zukunft immer vor Augen halten. 


\section{Literaturverzeichnis}

Abowd, Gregory D.; Dey, Anind K.; Brown, Peter J.; Davies, Nigel; Smith, Mark; Steggles, Pete (1999): Towards a Better Understanding of Context and ContextAwareness. In: Hans-Werner Gellersen (Hg.): Handheld and ubiquitous computing. Proceedings, Bd. 1707. Berlin [u.a.]: Springer (Lecture Notes in Computer Science, 1707), S. 304-307. Online verfügbar unter ftp://ftp.cc.gatech.edu/pub/gvu/tr/1999/99-22.pdf, zuletzt geprüft am 09.12.2018.

Adobe Systems Software Ireland Limited (2018): Kostenlose UI-Kits, Icons und Plugins. Adobe XD-Ressourcen. Dublin. Online verfügbar unter https://www.adobe.com/de/products/xd/resources.html\#panel-3, zuletzt geprüft am 08.12.2018.

Agrawal, Lokesh; Toshniwal, Durga (2013 - 2013): Smart Phone Based Indoor Pedestrian Localization System. In: 2013 13th International Conference on Computational Science and Its Applications. 2013 13th International Conference on Computational Science and Its Applications (ICCSA). Ho Chi Minh City, Vietnam, 24.06.2013 - 27.06.2013: IEEE, S. 137-143. Online verfügbar unter https://ieeexplore.ieee.org/document/6681111, zuletzt geprüft am 09.12.2018.

Al Nuaimi, Klaithem; Kamel, Hesham (2011): A survey of indoor positioning systems and algorithms. In: Innovations '11. 7th International Conference on Innovations in Information Technology : April 25-27, 2011, Abu Dhabi, United Arab Emirates. 2011 International Conference on Innovations in Information Technology (IIT). Abu Dhabi, United Arab Emirates, 4/25/2011 - 4/27/2011. Piscataway, N.J.: IEEE, S. 185-190. Online verfügbar unter https://ieeexplore.ieee.org/document/5893813, zuletzt geprüft am 09.12.2108.

Amundson, Isaac; Koutsoukos, Xenofon D. (2009): A Survey on Localization for Mobile Wireless Sensor Networks. In: Richard Fuller (Hg.): Mobile entity localization and tracking in GPS less environnments. Second international workshop ; proceedings, Bd. 5801. Berlin, Heidelberg, New York, NY: Springer (Lecture Notes in Computer Science, vol. 5801), S. 235-254. Online verfügbar unter https://www.researchgate.net/publication/225114031_A_Survey_on_Localization_for_Mobile_Wireless_Sensor_Networks, zuletzt geprüft am 09.12.2018. 
Angelis, Alessio de; Moschitta, Antonio; Comuniello, Antonella (2017): TDoA based positioning using ultrasound signals and wireless nodes. In: I'MTC 2017 IEEE International Instrumentation and Measurement Technology Conference. 2017 proceedings papers : May 22-25, 2017, Politecnico di Torino, Torino, Italy. 2017 IEEE International Instrumentation and Measurement Technology Conference (I2MTC). Torino, Italy, 5/22/2017 - 5/25/2017. Piscataway, NJ: IEEE, S. 1-6. Online verfügbar unter https://ieeexplore.ieee.org/document/7969873, zuletzt geprüft am 09.12.2018.

Apple Inc. (2018a): App Store. Cupertino, CA. Online verfügbar unter https://developer.apple.com/support/app-store/, zuletzt geprüft am 09.08.2018.

Apple Inc. (2018b): iBeacon. Cupertino, CA. Online verfügbar unter https://developer.apple.com/ibeacon/, zuletzt geprüft am 09.12.2018.

Apple Inc. (2018c): iOS 11. Cupertino, CA. Online verfügbar unter https://www.apple.com/de/ios/ios-11/, zuletzt geprüft am 09.08.2018.

AT\&T Laboratories Cambridge (Hg.) (2005): The Bat Ultrasonic Location System. Online verfügbar unter https://www.cl.cam.ac.uk/research/dtg/attarchive/bat/, zuletzt aktualisiert am 01.07.2005, zuletzt geprüft am 09.12.2018.

Bauer, H. H.; Haber, T. E.; Reichardt, T.; Bökamp, M. (2009): Konsumentenakzeptanz von Location Based Services. In: Hans H. Bauer, Melchior D. Bryant und Thorsten Dirks ( $\mathrm{Hg}$.$) : Erfolgsfaktoren des Mobile Marketing. Berlin, Heidelberg:$ Springer Berlin Heidelberg, S. 205-220. Online verfügbar unter https://link.springer.com/chapter/10.1007/978-3-540-85296-4_12, zuletzt geprüft am 09.12.2018.

Beineke, Julius; Spier, Alexander (2018): Das große Warten. Android-Smartphones im Update-Check. In: C't : Magazin für Computertechnik (ct) 2018 (16), S. 120123.

Bendel, Oliver: Wearables. Springer Fachmedien Wiesbaden GmbH. Wiesbaden. Online verfügbar unter https://wirtschaftslexikon.gabler.de/definition/wearables54088, zuletzt geprüft am 13.12.2018.

Bender, Hanno (2018): Sparkassen starten mobil durch. Frankfurt a.M. Online verfügbar unter http://www.bargeldlosblog.de/sparkassen-starten-mobil- 
durch/\#more-3726, zuletzt aktualisiert am 29.07.2018, zuletzt geprüft am 09.12.2018.

Berndt, Ulrike (2012): Wirtschaftswissenschaftliche Lehrbücher als E-Book über QRCode finden. Hg. v. Freie Universität Berlin. Berlin. Online verfügbar unter https://blogs.fu-berlin.de/bibliotheken/2012/05/11/wirtschaftswissenschaftlichelehrbucher-als-e-book-uber-qr-code-finden/, zuletzt aktualisiert am 11.05.2012, zuletzt geprüft am 21.07.2018.

Bitkom e.V. (Hg.) (2017): Zukunft der Consumer Technology - 2017. Marktentwicklung, Trends, Mediennutzung, Technologien, Geschäftsmodelle. Online verfügbar unter https://www.bitkom.org/sites/default/files/file/import/170901-CT-Studie-online.pdf, zuletzt geprüft am 08.12.2018.

Böhner, Dörte (2012): Location Based Services? Jena. Online verfügbar unter http://blog.bibliothekarisch.de/blog/2012/04/20/location-based-services/, zuletzt aktualisiert am 20.04.2012, zuletzt geprüft am 16.07.2018.

Bootstrap. Online verfügbar unter https://getbootstrap.com/, zuletzt geprüft am 08.12.2018.

Brás, Luis; Carvalho, Nuno Borges; Pinho, Pedro; Kulas, Lukasz; Nyka, Krzysztof (2012): A Review of Antennas for Indoor Positioning Systems. In: International Journal of Antennas and Propagation 2012 (6), S. 1-14. DOI:

$10.1155 / 2012 / 953269$.

Carrillo, Daniel; Moreno, Victoria; Úbeda, Benito; Skarmeta, Antonio F. (2015): MagicFinger. 3D Magnetic Fingerprints for Indoor Location. In: Sensors (Basel, Switzerland) 15 (7), S. 17168-17194. DOI: 10.3390/s150717168.

Ceynowa, Klaus (2016): „Leuchtfeuer“ in der Bayerischen Staatsbibliothek. Beacons-Technologie zur digitalen Indoor-Navigation für Bibliotheksbesucher. In: BFB (10), S. 14-17, zuletzt geprüft am 09.12.2018.

Chow, Chi-Wai; Chen, Chung-Yen; Chen, Shih-Hao (2015): Visible light communication using mobile-phone camera with data rate higher than frame rate. In: Opt. Express 23 (20), S. 26080. DOI: 10.1364/OE.23.026080. 
Cooper, Alan (2018): The origin of personas. San Francisco, CA, Brooklyn, NY. Online verfügbar unter https://www.cooper.com/journal/2008/05/the_origin_of_personas?, zuletzt aktualisiert am 15.05.2018, zuletzt geprüft am 08.12.2018.

Correa, Alejandro; Barcelo, Marc; Morell, Antoni; Vicario, Jose Lopez (2017): A Review of Pedestrian Indoor Positioning Systems for Mass Market Applications. In: Sensors (Basel, Switzerland) 17 (8). DOI: 10.3390/s17081927.

Crockford, Douglas: Einführung in JSON. Online verfügbar unter https://www.json.org/json-de.html, zuletzt geprüft am 13.12.2018.

DATACOM Buchverlag GmbH (2013): UWB (ultra wideband). Peterskirchen. Online verfügbar unter https://www.itwissen.info/UWB-ultra-wideband-Ultra-Breitband.html, zuletzt aktualisiert am 17.11.2013, zuletzt geprüft am 24.12.2018.

DATACOM Buchverlag GmbH (Hg.) (2014): Nahfeldkommunikation. Online verfügbar unter https://www.itwissen.info/Nahfeldkommunikation-near-field-communication-NFC.html, zuletzt aktualisiert am 01.11.2014, zuletzt geprüft am 09.12.2018.

DATACOM Buchverlag GmbH (Hg.) (2017): Geofencing. Online verfügbar unter https://www.itwissen.info/Geofencing-geofencing.html, zuletzt aktualisiert am 23.04.2017, zuletzt geprüft am 08.12.2108.

Deeg Christoph (2017): Wie Gamification die Bibliotheksarbeit verändern könnte. In: BIT online : Bibliothek, Information, Technologie (4), S. 315f. Online verfügbar unter https://www.b-i-t-online.de/heft/2017-04-nachrichtenbeitrag-deeg.pdf, zuletzt geprüft am 08.12.2018.

Dierolf, Uwe (2017): BibTip-Beacon. ein neuer ortsbezogener Informationskanal an der KIT-Bibliothek. In: BIT online : Bibliothek, Information, Technologie 20 (5), S. 371-382. Online verfügbar unter https://publikationen.bibliothek.kit.edu/1000075994/4510351, zuletzt geprüft am 08.12.2018.

Dierolf, Uwe (2018): Projekt gescheitert - viel gelernt. In: BuB : Forum Bibliothek und Information 70 (5), S. 256-260. Online verfügbar unter https://publikationen.bibliothek.kit.edu/1000082823/13353514, zuletzt geprüft am 08.12.2018.

Dierstein, Rüdiger (2003): Begriffe. Definitionen und Erklärungen. Hg. v. Ulmer Akademie für Datenschutz und IT-Sicherheit e.V. Oberpfaffenhofen. Online verfügbar 
unter http://wwwbayer.in.tum.de/lehre/WS2003/ITS-dierstein/DefDV03.pdf, zuletzt aktualisiert am 01.2003, zuletzt geprüft am 09.12.2018.

Dübbel, Nadine (2015): Phablet. cocodibu GmbH. München. Online verfügbar unter http://www.digitalwiki.de/phablet/, zuletzt aktualisiert am 23.07.2015, zuletzt geprüft am 13.12.2018.

ECMA International (2017): Standard ECMA-404. The JSON Data Interchange Syntax. Online verfügbar unter http://www.ecma-international.org/publications/files/ECMA-ST/ECMA-404.pdf, zuletzt aktualisiert am 12.2017, zuletzt geprüft am 13.12.2018.

Estimote, Inc. (Hg.) (2018): Estimote. Online verfügbar unter https://estimote.com/, zuletzt geprüft am 09.12.2018.

Farid, Zahid; Nordin, Rosdiadee; Ismail, Mahamod (2013): Recent Advances in Wireless Indoor Localization Techniques and System. In: Journal of Computer Networks and Communications 2013, S. 1-12. DOI: 10.1155/2013/185138.

Feigl, Maximilian (2018): Deutschland bei Location-based Services wieder führend. Hg. v. GFM Nachrichten. Erding. Online verfügbar unter http://www.gfm-nachrichten.de/news/aktuelles/article/deutschland-bei-location-based-services-wieder-fuehrend.html, zuletzt aktualisiert am 23.10.2018, zuletzt geprüft am 08.12.2018.

Fernandez, Jean-Claude; Mounier, Laurent; Pachon, Cyril (2005): A Model-Based Approach for Robustness Testing. In: Ferhat Khendek (Hg.): Testing of communicating systems. Proceedings, Bd. 3502. Berlin: Springer (Lecture Notes in Computer Science, 3502), S. 333-348. Online verfügbar unter https://www.researchgate.net/publication/221046918_A_Model-Based_Approach_for_Robustness_Testing, zuletzt geprüft am 09.12.2018.

Fischer, Philippe (2017): Das bedeuten WLAN N, AC und 5 Ghz. WLAN ist nicht gleich WLAN. Hg. v. Axel Springer SE. Berlin. Online verfügbar unter https://www.techbook.de/easylife/internet-of-things/was-bedeutet-wlan-n-ac5ghz, zuletzt aktualisiert am 16.11.2017, zuletzt geprüft am 09.12.2018.

Garrett, Jesse James (2005): Ajax: A New Approach to Web Applications. Adaptive Path. San Francisco, CA. Online verfügbar unter 
https://web.archive.org/web/20080702075113/http://www.adaptivepath.com/ideas/essays/archives/000385.php, zuletzt aktualisiert am 18.02.2005, zuletzt geprüft am 13.12.2018.

Gartner Says Worldwide Sales of Smartphones Recorded First Ever Decline During the Fourth Quarter of 2017 (2018). Egham. Online verfügbar unter https://www.gartner.com/newsroom/id/3859963, zuletzt aktualisiert am 22.02.2018, zuletzt geprüft am 08.12.2018.

Goldmedia GmbH Strategy (Hg.) (2014): Location-based Services Monitor 2014. Angebote, Nutzung und lokale Werbemarktpotenziale ortsbezogener, mobiler Dienste in Deutschland (Gesamtstudie). Online verfügbar unter https://www.blm.de/files/pdf1/140512_Location-based_Services_Monitor_2014.pdf, zuletzt geprüft am 08.12.2018.

Google LLC (2017): Text Recognition API Overview. Mountain View, CA. Online verfügbar unter https://developers.google.com/vision/android/text-overview, zuletzt aktualisiert am 20.12.2017, zuletzt geprüft am 09.12.2018.

Google LLC (2018a): <uses-sdk>. Mountain View, CA. Online verfügbar unter https://developer.android.com/guide/topics/manifest/uses-sdk-element, zuletzt aktualisiert am 20.11.2018, zuletzt geprüft am 08.12.2018.

Google LLC (2018b): App bars: bottom. Usage. Mountain View, CA. Online verfügbar unter https://material.io/design/components/app-bars-bottom.html\#usage, zuletzt aktualisiert am 30.10.2018, zuletzt geprüft am 08.12.2018.

Google LLC (2018c): Distribution dashboard. Mountain View, CA. Online verfügbar unter https://developer.android.com/about/dashboards/, zuletzt aktualisiert am 23.07.2018, zuletzt geprüft am 09.08.2018.

Google LLC (2018d): Eddystone format. Mountain View, CA. Online verfügbar unter https://developers.google.com/beacons/eddystone, zuletzt aktualisiert am 27.04.2018, zuletzt geprüft am 09.12.2018.

Google LLC (2018e): Get Started with the Mobile Vision iOS API. Mountain View, CA. Online verfügbar unter https://developers.google.com/vision/ios/gettingstarted, zuletzt aktualisiert am 20.04.2018, zuletzt geprüft am 09.12.2018. 
Google LLC (2018f): Make apps more accessible. Mountain View, CA. Online verfügbar unter https://developer.android.com/guide/topics/ui/accessibility/apps, zuletzt aktualisiert am 14.08.2018, zuletzt geprüft am 08.12.2018.

Google LLC (2018g): Overview of Google Play Services. Mountain View, CA. Online verfügbar unter https://developers.google.com/android/guides/overview, zuletzt aktualisiert am 23.08.2018, zuletzt geprüft am 09.12.2018.

Google LLC (2018h): See and Understand Text using OCR with Mobile Vision Text API for Android. Mountain View, CA. Online verfügbar unter https://codelabs.developers.google.com/codelabs/mobile-vision-ocr/\#0, zuletzt aktualisiert am 08.05.2018, zuletzt geprüft am 09.12.2018.

Google LLC (2018i): The type system. Use typography to present your design and content as clearly and efficiently as possible. Mountain View, CA. Online verfügbar unter https://material.io/design/typography/the-type-system.html\#, zuletzt aktualisiert am 30.10.2018, zuletzt geprüft am 08.12.2018.

Google LLC (2018j): Volley overview. Mountain View, CA. Online verfügbar unter https://developer.android.com/training/volley/, zuletzt aktualisiert am 17.07.2018, zuletzt geprüft am 08.12.2018.

Google LLC (2018k): Wi-Fi scanning overview. Mountain View, CA. Online verfügbar unter https://developer.android.com/guide/topics/connectivity/wifi-scan, zuletzt aktualisiert am 04.10.2018, zuletzt geprüft am 09.12.2018.

Gradle Inc. (2018): Gradle. features. San Francisco, CA. Online verfügbar unter https://gradle.org/features/, zuletzt aktualisiert am 11.12.2018, zuletzt geprüft am 13.12.2018.

Griebel, Rolf; Schäffler, Hildegard; Söllner, Konstanze; Frantz, Eva Anne (Hg.) (2015): Praxishandbuch Bibliotheksmanagement. Berlin, München, Boston: de Gruyter Saur (Reference). Online verfügbar unter https://www.degruyter.com/viewbooktoc/product/203487, zuletzt geprüft am 09.12.2018.

Grohnert, Ana-Christina (2016): EY Studentenstudie 2016. Studenten in Deutschland: Werte, Ziele, Perspektiven. Hg. v. Ernst \& Young GmbH Wirtschaftsprüfungsgesellschaft. Stuttgart. Online verfügbar unter https://www.ey.com/Publication/vwLUAssets/EY-studentenstudie-2016-werte-ziele-perspektiven/\$FILE/EY- 
studentenstudie-2016-werte-ziele-perspektiven.pdf, zuletzt aktualisiert am 07.2016, zuletzt geprüft am 08.12.2018.

Grüner, Sebastian (2017): Google will Android-Updates revolutionieren. Project Treble. Hg. v. Golem Media GmbH. Berlin. Online verfügbar unter https://www.golem.de/news/project-treble-google-will-android-updates-revolutionieren-1705-127803.html, zuletzt aktualisiert am 13.05.2017, zuletzt geprüft am 08.12.2018.

Gu, Yanying; Lo, Anthony; Niemegeers, Ignas (2009): A survey of indoor positioning systems for wireless personal networks. In: IEEE Commun. Surv. Tutorials 11 (1), S. 13-32. DOI: 10.1109/SURV.2009.090103.

Gutierrez, Juan F.; Hunt, Charles E.; Quintero, Jesus M. (2014): Visible Light Communication LED based Luminaire for General Lighting. State of Art. In: XII Conferência Panamericana de Iluminação, S. 163-170. Online verfügbar unter https://pdfs.semanticscholar.org/32a4/853c341ea508a7a89224f9fdc49c006e851f.pdf, zuletzt geprüft am 09.12.2018.

Haas, Markus (2018): Smartphone-Markt. Konjunktur und Trends. Hg. v. Bitkom e.V. Berlin. Online verfügbar unter https://www.bitkom.org/sites/default/files/file/import/Bitkom-Pressekonferenz-Smartphone-Markt-22-02-2018-Praesentation-final.pdf, zuletzt aktualisiert am 22.08.2018, zuletzt geprüft am 08.12.2018.

Hahn, Jim (2017): The Internet of Things. Mobile technology and location services in libraries. Chicago, IL: ALA TechSource (Library technology reports, vol. 53, no. 1). Online verfügbar unter https://journals.ala.org/index.php/Itr/issue/download/621/384, zuletzt geprüft am 08.12.2018.

Harle, Robert (2013): A Survey of Indoor Inertial Positioning Systems for Pedestrians. In: IEEE Commun. Surv. Tutorials 15 (3), S. 1281-1293. DOI: 10.1109/SURV.2012.121912.00075.

Hery-Moßmann, Nicole (2017): Wearables - was ist das eigentlich? CHIP Digital $\mathrm{GmbH}$. München. Online verfügbar unter https://praxistipps.chip.de/wearableswas-ist-das-eigentlich_95328, zuletzt aktualisiert am 21.08.2017, zuletzt geprüft am 13.12.2018. 
Holt, Eva-Maria; Winter, Dominique; Thomaschewski, Jörg (2010): Personas als Werkzeug in modernen Softwareprojekten. Online verfügbar unter https://de.slideshare.net/designik/personas-als-werkzeug-in-modernen-softwareprojekten, zuletzt aktualisiert am 11.09.2010, zuletzt geprüft am 08.12.2018.

Huang, Haosheng; Gartner, Georg (2018): Current Trends and Challenges in Location-Based Services. In: IJGI 7 (6), S. 1-199. DOI: 10.3390/ijgi7060199.

identWERK GmbH: 1D-Barcode und 2D-Barcode im Vergleich. Bad Rappenau. Online verfügbar unter https://www.identwerk.de/technologie/1d-barcode-und-2dbarcode-im-vergleich, zuletzt geprüft am 24.12.2018.

IEEE Std 802.11ah-2016 (Amendment to IEEE Std 802.11-2016, as amended by IEEE Std 802.11ai-2016). IEEE Standard for Information technology--Telecommunications and information exchange between systems - Local and metropolitan area networks--Specific require (2017). S.I.: IEEE. Online verfügbar unter http://ieeexplore.ieee.org/servlet/opac?punumber=7920362.

Infopark AG: CMS Fiona. Umsetzung interaktiver und dynamischer Webauftritte. Berlin. Online verfügbar unter https://infopark.com/de/plattform/cms-fiona, zuletzt geprüft am 26.12.2018.

inside-intermedia GmbH (2018): Handy-Finder. Brühl. Online verfügbar unter https://www.inside-handy.de/handy-finder, zuletzt geprüft am 08.12.2018.

International vocabulary of metrology. Basic and general concepts associated terms (2012). 3rd edition: JCGM. Online verfügbar unter https://www.bipm.org/utils/common/documents/jcgm/JCGM_200_2012.pdf. Introduction. Online verfügbar unter https://getbootstrap.com/docs/4.1/gettingstarted/introduction/, zuletzt geprüft am 08.12.2018.

Janssen, Jan-Keno; Porteck, Stefan: Appbezahlen. Smartphone-Bezahl-Apps im Test. In: C't : Magazin für Computertechnik (ct) 2018 (16), S. 68-72.

Jiao, Jichao; Li, Fei; Deng, Zhongliang; Liu, Wen (2016): An indoor positioning method based on wireless signal and image. In: Yaoli Wang (Hg.): CISP-BMEI 2016. Proceedings, 2016 9th International Congress on Image and Signal Processing, BioMedical Engineering and Informatics : 15-17 October 2016, Datong, China. 
2016 9th International Congress on Image and Signal Processing, BioMedical Engineering and Informatics (CISP-BMEl). Datong, China, 10/15/2016 - 10/17/2016. [Piscataway, New Jersey]: IEEE, S. 656-660. Online verfügbar unter https://ieeexplore.ieee.org/document/7852791, zuletzt geprüft am 09.12.2018.

Joachim Herz Stiftung: Beugung und Interferenz. Hamburg. Online verfügbar unter https://www.leifiphysik.de/optik/beugung-und-interferenz, zuletzt geprüft am 14.12.2018.

jQuery Foundation (2018): jQuery. write less, do more. Online verfügbar unter https://jquery.com/, zuletzt geprüft am 08.12.2018.

Kahlkopf, Alexander (Hg.) (2018a): iconmonstr. Online verfügbar unter https://iconmonstr.com/, zuletzt geprüft am 08.12.2018.

Kahlkopf, Alexander (Hg.) (2018b): License Agreement. Online verfügbar unter https://iconmonstr.com/license/, zuletzt geprüft am 08.12.2018.

Karlsruher Institut für Technologie (Hg.) (2009): BibTip macht sich selbstständig. Online verfügbar unter https://www.kit.edu/kit/pi_2009_111.php, zuletzt aktualisiert am 05.05.2009, zuletzt geprüft am 09.12.2018.

Karlsruher Institut für Technologie (Hg.) (2013): Lehrbuchsammlung der KIT-Bibliothek Süd jetzt mit QR-Codes. Online verfügbar unter http://blog.bibliothek.kit.edu/kit_bib_news/index.php/2013/07/03/lehrbuchsammlung-der-kit-bibliothek-sud-jetzt-mit-qr-codes/, zuletzt aktualisiert am 03.07.2013, zuletzt geprüft am 08.12.2018.

Karlsruher Institut für Technologie (Hg.) (2017): Einführung der Infoapp „OnSitelnfo“. Online verfügbar unter http://blog.bibliothek.kit.edu/kit_bib_news/index.php/2017/11/20/einfuehrung-der-infoapp-onsiteinfo/, zuletzt aktualisiert am 20.11.2017, zuletzt geprüft am 08.12.2018.

Ketamo, Harri; Koivisto, Antti; Hammais, Eero (2014): Combining the Dimension of Written and Digital Media in a NFC-based Non-Linear Adventure Game for Children. Online verfügbar unter http://library.ifla.org/872/1/168-koivisto-en.pdf, zuletzt aktualisiert am 24.06.2014, zuletzt geprüft am 08.12.2018. 
Kim, Seong-Eun; Kim, Yong; Yoon, Jihyun; Kim, Eung Sun (2012): Indoor positioning system using geomagnetic anomalies for smartphones. In: C. Rizos (Hg.): 2012 International Conference on Indoor Positioning and Indoor Navigation. 13-15 November, 2012, Sydney, Australia. 2012 International Conference on Indoor Positioning and Indoor Navigation (IPIN). Sydney, Australia, 11/13/2012 - 11/15/2012. Piscataway, NJ: IEEE, S. 1-5. Online verfügbar unter https://www.researchgate.net/publication/261155828_Indoor_positioning_system_using_geomagnetic_anomalies_for_smartphones, zuletzt geprüft am 09.12.2018.

Kontakt.io, Inc. (2018): BLE Beacons and Tags. New York. Online verfügbar unter https://kontakt.io/ble-beacons-tags/, zuletzt geprüft am 09.12.2018.

Krautz, Sebastian (2014): Einflüsse von NFC-Smartphones auf das RFID- Bibliothekssystem // Einflüsse von NFC-Smartphones auf das RFID- Bibliothekssystem : eine Analyse des Bedrohungspotentials durch NFC-Smartphones und Beschreibung von möglichen Gegenmaßnahmen. eine Analyse des Bedrohungspotentials durch NFC-Smartphones und Beschreibung von möglichen Gegenmaßnahmen. In: Frank Seeliger (Hg.): RFID für Bibliothekare. Ein Vademecum. 3. Aufl. Berlin: News \& Media, S. 209-221. Online verfügbar unter https://opus4.kobv.de/opus4th-wildau/frontdoor/index/index/docld/276, zuletzt geprüft am 09.12.2018.

Kumar, Arvind (2016): Sensors in a Smartphone and There Uses. Online verfügbar unter http://www.gadgetinfo.in/2016/10/sensors-in-smartphone-and-there-uses.html, zuletzt aktualisiert am 09.10.2016, zuletzt geprüft am 08.12.2018.

Küng, Roland (2009): Kapitel 2 Ausbreitung von Radiowellen I. ZHAW Zürcher Hochschule für Angewandte Wissenschaften. Winterthur. Online verfügbar unter https://home.zhaw.ch/kunr/NTM1/unterlagen/Kap2_Propagation_2009v1_2.pdf, zuletzt aktualisiert am 2009, zuletzt geprüft am 14.12.2018.

Künneth, Thomas (2018): Android 8. Das Praxisbuch für Java-Entwickler. 5., aktualisierte Auflage. Bonn: Rheinwerk Verlag (Rheinwerk Computing). Online verfügbar unter http://www.rheinwerk-verlag.de/android-8_4564/.

Kushwaha, Amit; Kushwaha, Vineet (2011): Location Based Services using Android Mobile Operating System. In: International Journal of Advances in Engineering \& Technology 1, S. 14-20. Online verfügbar unter 
http://www.ijaet.org/media/Location-Based-Services-using-Android-mobile-Operating-System-Copyright-IJAET.pdf, zuletzt geprüft am 08.12.2018.

Landolt, Niklaus; Reto, List (2016): In der umgebauten Bibliothek Münstergasse ERessourcen sichtbar machen. Hg. v. Universität Bern. Bern. Online verfügbar unter http://www.bis.ch/fileadmin/ressourcen/kongress/2016_luzern/Referate/Landolt_List_Muenstergasse_e-Ressourcen.pdf, zuletzt aktualisiert am 02.09.2016, zuletzt geprüft am 08.12.2018.

Lang, Sabine: Empirische Forschungsmethoden. Skript zur Lehrveranstaltung. Online verfügbar unter https://www.uni-trier.de/fileadmin/fb1/prof/PAD/SP2/Allgemein/Lang_Skript_komplett.pdf, zuletzt geprüft am 08.12.2018.

Lazik, Patrick; Rowe, Anthony (2012): Indoor pseudo-ranging of mobile devices using ultrasonic chirps. In: Rasit Eskicioglu, Andrew Campbell und Koen Langendoen $(\mathrm{Hg}$.): SenSys 2012. Proceedings of the 10th ACM conference on embedded networked sensor systems, Toronto, Canada, 06-09.11.2012. the 10th ACM Conference. Toronto, Ontario, Canada, 11/6/2012 - 11/9/2012. International Conference on Embedded Networked Sensor Systems. New York (NY): A.C.M, S. 99. Online verfügbar unter http://wise.ece.cmu.edu/resources/publications/sensys_12.pdf, zuletzt geprüft am 09.12.2018.

Li, Binghao; Gallagher, Thomas; Dempster, Andrew G.; Rizos, Chris (2012): How feasible is the use of magnetic field alone for indoor positioning? In: C. Rizos (Hg.): 2012 International Conference on Indoor Positioning and Indoor Navigation. 13-15 November, 2012, Sydney, Australia. 2012 International Conference on Indoor Positioning and Indoor Navigation (IPIN). Sydney, Australia, 11/13/2012 11/15/2012. Piscataway, NJ: IEEE, S. 1-9. Online verfügbar unter https://www.researchgate.net/publication/261310654_How_feasible_is_the_use_of_magnetic_field_alone_for_indoor_positioning, zuletzt geprüft am 09.12.2018.

List, Reto; Kirgus, Isabelle (2017): Standortbasierte Online-Informationen vermitteln. cUBe, ein Projekt der Universitätsbibliothek Bern. In: o-bib - das offene Bibliotheksjournal (4), S. 63-70. DOI: 10.5282/O-BIB/2017H4S63-70.

List, Reto [reto.list@ub.unibe.ch] (2018): AW: Projekt cUBe, 22.06.2018. E-Mail an kai.barth87@googlemail.com (Barth Kai). 
Liu, Hui; Darabi, Houshang; Banerjee, Pat; Liu, Jing (2007): Survey of Wireless Indoor Positioning Techniques and Systems. In: IEEE Trans. Syst., Man, Cybern. C 37 (6), S. 1067-1080. DOI: 10.1109/TSMCC.2007.905750.

Liu, Kaixu; Motta, Gianmario; Dong, Juncheng (2017 - 2017): Wi-Fi-Aided Magnetic Field Positioning with Floor Estimation in Indoor Multi-Floor Navigation Services. In: 2017 IEEE International Congress on Intemet of Things (ICIOT). 2017 IEEE International Congress on Internet of Things (ICIOT). Honolulu, HI, USA, 25.06.2017 - 30.06.2017: IEEE, S. 129-136. Online verfügbar unter https://www.researchgate.net/publication/319888274_Wi-Fi-Aided_Magnetic_Field_Positioning_with_Floor_Estimation_in_Indoor_Multi-Floor_Navigation_Services, zuletzt geprüft am 09.12.2018.

Lopez, Carola (2013): Studie zur Bekanntheit und Nutzung von Location-Based-Services (LBS) bei Besitzern und Nicht-Besitzern mobiler Devices. Hg. v. Bundesverband Digitale Wirtschaft (BVDW) e.V. Berlin. Online verfügbar unter https://www.bvdw.org/presseserver/bvdw_mobile_datendienste/chartband_bvdw_mobile_datendienste.pdf, zuletzt aktualisiert am 03.2013, zuletzt geprüft am 08.12.2018.

Ludwig-Maximilians-Universität München (Hg.): Geschichte des Gebäudes. Online verfügbar unter https://www.ub.uni-muenchen.de/bibliotheken/fachbibliotheken_uebersicht/philologicum/geschichte-des-gebaeudes/index.html, zuletzt geprüft am 08.12.2018.

Ludwig-Maximilians-Universität München: Portrait. Fakultät für Sprach- und Literaturwissenschaften. München. Online verfügbar unter https://www.sprach-und-literaturwissenschaften.uni-muenchen.de/fakultaet/portrait/index.html, zuletzt geprüft am 08.12.2018.

Ludwig-Maximilians-Universität München (Hg.) (2013): „Planungen zum Philologicum können beginnen“. Seehofer an der LMU. Online verfügbar unter https://www.uni-muenchen.de/aktuelles/news/2013/seehofer.html, zuletzt aktualisiert am 10.04.2013, zuletzt geprüft am 08.12.2018.

Ludwig-Maximilians-Universität München (Hg.) (2016): Grundsteinlegung für die Fachbibliothek Philologicum. Am 24. November 2016 findet der offizielle 
Startschuss zum Neubau statt. Online verfügbar unter https://www.ub.uni-muenchen.de/aktuelles/archiv/meldungen/grundsteinlegung-philologicum/index.html, zuletzt aktualisiert am 24.11.2016, zuletzt geprüft am 08.12.2018.

Ludwig-Maximilians-Universität München (Hg.) (2017a): Das Philologicum feiert Richtfest. Neue Fachbibliothek der LMU. Online verfügbar unter https://www.unimuenchen.de/aktuelles/news/2017/philologicum_richtfest.html, zuletzt aktualisiert am 25.07.2018, zuletzt geprüft am 08.12.2018.

Ludwig-Maximilians-Universität München (Hg.) (2017b): Das Philologicum hat Richtfest gefeiert. Der Abschluss der Rohbauarbeiten wurde am 25.07.2017 mit einem Richtfest gefeiert. Online verfügbar unter https://www.ub.uni-muenchen.de/aktuelles/archiv/meldungen/philologicum-richtfest/index.html, zuletzt aktualisiert am 25.07.2017, zuletzt geprüft am 08.12.2018.

Ludwig-Maximilians-Universität München (Hg.) (2017c): Fachbibliothek Philologicum. Online verfügbar unter https://www.ub.uni-muenchen.de/bibliotheken/fachbibliotheken_uebersicht/philologicum/index.html, zuletzt geprüft am 08.12.2018.

Ludwig-Maximilians-Universität München (Hg.) (2018): Zahlen und Fakten. Online verfügbar unter https://www.uni-muenchen.de/ueber_die_Imu/zahlen_fakten/index.html, zuletzt geprüft am 08.12.2018.

Ludwig-Maximilians-Universität München Universitätsbibliothek (Hg.) (2018): Jahresbericht 2017. Online verfügbar unter https://epub.ub.uni-muenchen.de/57395/1/Jahresbericht_2017_Universitaetsbibliothek_LMU.pdf, zuletzt aktualisiert am 08.2018, zuletzt geprüft am 08.12.2018.

Malchev, lliyan (2017): Here comes Treble: A modular base for Android. Google LLC. Cupertino, CA. Online verfügbar unter https://android-developers.googleblog.com/2017/05/here-comes-treble-modular-base-for.html, zuletzt aktualisiert am 12.05.2017, zuletzt geprüft am 08.12.2018.

Mandal, Atri; Lopes, Cristina V.; Givargis, Tony; Haghighat, Amir; Jurdak, Raja; Baldi, Pierre (2005): Beep: 3D indoor positioning using audible sound. In: 2005 Second IEEE Consumer Communications and Networking Conference, 2005. CCNC ; 3-6 Jan. 2005, [Caesar's Palace, Las Vegas, Nevada, USA]. Second IEEE Consumer Communications and Networking Conference, 2005. CCNC. 2005. Las 
Vegas, NV, USA, 3-6 Jan. 2005. Consumer Communications and Networking Conference; IEEE Consumer Communications and Networking Conference; CCNC. Piscataway, NJ: IEEE Operations Center, S. 348-353. Online verfügbar unter https://www.ics.uci.edu/ givargis/pubs/C25.pdf, zuletzt geprüft am 09.12.2018.

Massmann, Dirk (2018): Arbeiten mit JSON. Mozilla Foundation. Mountain View, CA. Online verfügbar unter https://developer.mozilla.org/de/docs/Learn/JavaScript/Objects/JSON, zuletzt aktualisiert am 21.08.2018, zuletzt geprüft am 13.12.2018.

Mautz, Rainer (2012): Indoor positioning technologies. Zürich: ETH Zurich (Research Collection). Online verfügbar unter https://www.research-collection.ethz.ch/handle/20.500.11850/54888, zuletzt geprüft am 09.12.2018.

Mees, Matthias (2015): Bootstrap - Segen oder Fluch? UI-Frameworks. Hg. v. Jens Grochtdreis. Mainz. Online verfügbar unter http://webkrauts.de/artikel/2015/bootstrap-segen-oder-fluch, zuletzt aktualisiert am 08.12.2015, zuletzt geprüft am 08.12.2018.

Meindl, Claudia (2013): User Stories in Scrum. Hg. v. AlphaNodes GmbH. München. Online verfügbar unter https://alphanodes.com/de/user-stories-scrum, zuletzt aktualisiert am 16.07.2013, zuletzt geprüft am 08.12.2018.

Meinel, Christoph; Sack, Harald (2012): Internetworking. Technische Grundlagen und Anwendungen. Berlin, Heidelberg: Springer-Verlag Berlin Heidelberg (X.media.press). Online verfügbar unter http://dx.doi.org/10.1007/978-3-540-92940-6, zuletzt geprüft am 09.12.2018.

Minor, Jens (2017): Android-Revolution: Googles Project Treble kann die Fragmentierung durch schnelle Updates beenden. Wien. Online verfügbar unter https://www.googlewatchblog.de/2017/11/android-revolution-das-project/, zuletzt aktualisiert am 26.11.2017, zuletzt geprüft am 08.12.2018.

mysql - sql injection prevention. Online verfügbar unter http://www.tizag.com/mysqlTutorial/mysql-php-sql-injection.php, zuletzt geprüft am 13.12.2018. 
NFC21 GmbH: NFC Sticker weiß \& schwarz. NFC Tags (NFC-Aufkleber / NFC-Sticker). Mülheim an der Ruhr. Online verfügbar unter https://www.nfc-tagshop.de/nfc-aufkleber/nfc-sticker-weiss-schwarz/?p=1, zuletzt geprüft am 24.09.2018.

Nielsen, Lene; Storgaard Hansen, Kira (2014): Personas is applicable. A Study On The Use of Personas In Denmark. In: Matt Jones, Philippe Palanque, Albrecht Schmidt und Tovi Grossman (Hg.): CHI 2014, one of a CHInd. Conference proceedings : Toronto, Canada, April 26 - May 1, 2014 ; the 32nd Annual ACM Conference on Human Factors in Computing Systems. the 32nd annual ACM conference. Toronto, Ontario, Canada, 4/26/2014 - 5/1/2014. Association for Computing Machinery; CHI Conference; Annual ACM Conference on Human Factors in Computing Systems. New York, NY: Assoc. for Computing Machinery, S. 16651674. Online verfügbar unter https://core.ac.uk/download/pdf/50527779.pdf, zuletzt geprüft am 09.12.2018.

Noertjahyana, Agustinus; Wijayanto, Ignatius Alex; Andjarwirawan, Justinus (2017 2017): Development of Mobile Indoor Positioning System Application Using Android and Bluetooth Low Energy with Trilateration Method. In: 2017 International Conference on Soft Computing, Intelligent System and Information Technology (ICSIIT). 2017 International Conference on Soft Computing, Intelligent System and Information Technology (ICSIIT). Denpasar, Bali, Indonesia, 26.09.2017 29.09.2017: IEEE, S. 185-189. Online verfügbar unter https://ieeexplore.ieee.org/document/8262565, zuletzt geprüft am 09.12.2018.

Nowicki, Michal; Rostkowska, Marta; Skrzypczynski, Piotr (2016): Indoor navigation using QR codes and WiFi signals with an implementation on mobile platform. In: Architectures Arrangements and Applications Signal Processing: Algorithms ( $\mathrm{Hg}$.): SPA 2016. Signal Processing: Algorithms, Architectures, Arrangements, and Applications : conference proceedings : Poznan, 21-23rd September 2016. 2016 Signal Processing: Algorithms, Architectures, Arrangements and Applications (SPA). Poznan, 9/21/2016 - 9/23/2016. Signal Processing: Algorithms, Architectures, Arrangements, and Applications; IEEE SPA Conference; SPA. [Piscataway, NJ]: IEEE, S. 156-161. Online verfügbar unter https://ieeexplore.ieee.org/document/7763605, zuletzt geprüft am 09.12.2018. 
NXP Semiconductors (Hg.) (20018): NFC TagWriter by NXP. Google LLC. Online verfügbar unter https://play.google.com/store/apps/details?id=com.nxp.nfc.tagwriter, zuletzt aktualisiert am 26.10.2018, zuletzt geprüft am 09.12.2018.

Oracle (2018): 14.1 Introduction to InnoDB. Redwood City, CA. Online verfügbar unter https://dev.mysql.com/doc/refman/5.6/en/innodb-introduction.html, zuletzt aktualisiert am 12.12.2018, zuletzt geprüft am 13.12.2018.

Ostermann, Klaus: Einführung in die Softwaretechnik. 8. Anfoderungsanalyse. Online verfügbar unter http://ps.informatik.uni-tuebingen.de/teaching/ss16/se/7_Anforderungsanalyse.pdf, zuletzt geprüft am 09.12.2018.

Ozdenizci, Busra; Coskun, Vedat; Ok, Kerem (2015): NFC internal: an indoor navigation system. In: Sensors (Basel, Switzerland) 15 (4), S. 7571-7595. DOI: $10.3390 / s 150407571$.

Porteck, Stefan (2018): Aus P wird Pie. Android 9 ist erschienen. In: C't : Magazin für Computertechnik (ct) 2018 (18), S. 29.

PSA Publishers Ltd. (Hg.) (2014): Fachbibliothek Philologicum LMU München. Online verfügbar unter https://www.german-architects.com/de/cukrowicz-nachbaur-architekten-bregenz/project/fachbibliothek-philologicum-Imu-munchen, zuletzt geprüft am 07.12.2018.

Puertolas-Montaez, Jose Antonio; Mendoza-Rodriguez, Adriana; Sanz-Prieto, Ivan (2013): Smart Indoor Positioning/Location and Navigation: A Lightweight Approach. In: IJIMAI 2 (2), S. 43. DOI: 10.9781/ijimai.2013.225.

Puussaar, Aare (2014): Indoor Positioning Using WLAN Fingerprinting with Post-Processing Scheme. Master Thesis. University of Tartu, Tartu. Physics Department. Online verfügbar unter http://kodu.ut.ee/ srirama/publications/theses/Indoor_Positioning_Using_WLAN_Fingerprinting_with_Post-Processing_Scheme_Aare_Puussaar_Final.pdf, zuletzt geprüft am 09.12.2018.

Sakpere, Wilson; Adeyeye Oshin, Michael; Mlitwa, Nhlanhla B. W. (2017): A State-ofthe-Art Survey of Indoor Positioning and Navigation Systems and Technologies. In: SACJ 29 (3), S. 145-197. DOI: 10.18489/sacj.v29i3.452. 
Schmidt, Florian (Hg.) (2018): Liste von Android-Versionen. Online verfügbar unter https://www.droidwiki.org/wiki/Liste_von_Android-Versionen, zuletzt aktualisiert am 05.09.2018, zuletzt geprüft am 08.12.2018.

Schmidt, Julia (2013): Gradle als Googles Build-System für Android. Heise Medien $\mathrm{GmbH} \&$ Co. KG. Hannover. Online verfügbar unter https://www.heise.de/developer/meldung/Gradle-als-Googles-Build-System-fuer-Android-1867576.html, zuletzt aktualisiert am 22.05.2013, zuletzt geprüft am 13.12.2018.

Schnabel, Patrick (Hg.): Bluetooth 1.0/1.1/1.2 (IEEE 802.15). Ludwigsburg. Online verfügbar unter http://www.elektronik-kompendium.de/sites/kom/0803301.htm, zuletzt geprüft am 09.12.2018.

Schnabel, Patrick (Hg.): Funktechnik (Grundlagen). Online verfügbar unter https://www.elektronik-kompendium.de/sites/kom/0810301.htm, zuletzt geprüft am 14.12.2018.

Schüller-Zwierlein, André (2014): Architektenwettbewerb für das Philologicum ist entschieden. In: BFB (4), S. 313-315. Online verfügbar unter https://www.bibliotheksforum-bayern.de/fileadmin/archiv/2014-4/PDF-Einzelbeitraege/BFB_0414_16_Schueller_V04.pdf, zuletzt geprüft am 08.12.2018.

Schwaber, Ken; Sutherland, Jeff (2018): The Scrum Guide. Online verfügbar unter https://www.scrumguides.org/scrum-guide.html, zuletzt aktualisiert am 23.02.2018, zuletzt geprüft am 08.12.2018.

SELFHTML e.V. (2018): JavaScript/Ajax. Hof Kreien. Online verfügbar unter https://wiki.selfhtml.org/wiki/JavaScript/Ajax, zuletzt aktualisiert am 09.10.2018, zuletzt geprüft am 13.12.2018.

Shahidi, Shervin; Valaee, Shahrokh (2015): GIPSy: Geomagnetic indoor positioning system for smartphones. In: Kyle O’Keefe, Adriano Moreira, Mark Petovello und Gérard Lachapelle (Hg.): 2015 International Conference on Indoor Positioning and Indoor Navigation. 13-16 October 2015, Banff, Alberta, Canada. 2015 International Conference on Indoor Positioning and Indoor Navigation (IPIN). Banff, AB, Canada, 10/13/2015 - 10/16/2015. [Piscataway, New Jersey]: IEEE, S. 1-7. Online verfügbar unter https://ieeexplore.ieee.org/document/7346761, zuletzt geprüft am 09.12.2018. 
Shao, Wenhua; Zhao, Fang; Wang, Cong; Luo, Haiyong; Muhammad Zahid, Tunio; Wang, Qu; Li, Dongmeng (2016): Location Fingerprint Extraction for Magnetic Field Magnitude Based Indoor Positioning. In: Journal of Sensors 2016, S. 1-16. DOI: 10.1155/2016/1945695.

Simon, Oliver (2018): EY Studentenstudie 2018. In welchen Branchen zieht es Studenten in Deutschland? Hg. v. Ernst \& Young GmbH Wirtschaftsprüfungsgesellschaft. Stuttgart. Online verfügbar unter https://www.ey.com/Publication/vwLUAssets/ey-studentenstudie-2018/\$FILE/ey-studentenstudie-2018.pdf, zuletzt aktualisiert am 07.2018, zuletzt geprüft am 08.12.2018.

Spiekermann, Sarah (op. 2004): General aspects of location-based services. In: Jochen $\mathrm{H}$. Schiller und Agnès Voisard (Hg.): Location-based services. San Francisco, Amsterdam, Paris: Morgan Kaufmann, S. 9-26. Online verfügbar unter http://citeseerx.ist.psu.edu/viewdoc/download;jsessionid=1A0F06A071618BE73C79AF06B182A7A3?doi=10.1.1.59.1289\&rep=rep1\&ty pe=pdf, zuletzt geprüft am 09.12.2018.

Sroke, Norbert (Hg.) (2018): QRCode Monkey. Der kostenlose QR Code Generator. Online verfügbar unter https://www.qrcode-monkey.com/de\#url, zuletzt geprüft am 09.12.2018.

Stabenau, Edlef (2010): QR Codes in der Bibliothek. Hg. v. Universitätsbibliothek Dortmund. Dortmund. Online verfügbar unter https://log.netbib.de/archives/2010/03/22/qr-codes-in-der-bibliothek/, zuletzt aktualisiert am 22.03.2010, zuletzt geprüft am 09.12.2018.

Stack Exchange, Inc. (Hg.) (2011): Can NFC tags be protected from being overwritten? Online verfügbar unter https://stackoverflow.com/questions/5669833/cannfc-tags-be-protected-from-being-overwritten, zuletzt aktualisiert am 10.10.2016, zuletzt geprüft am 09.12.2018.

Statista (2017): Leben der Studenten in Deutschland. Dossier. Hamburg. Online verfügbar unter https://de.statista.com/statistik/studie/id/54729/dokument/lebender-studenten-in-deutschland/, zuletzt geprüft am 08.12.2018.

Statista (2018): Vorbildung der Studierenden in Deutschland in den Jahren von 2003 bis 2012. Hamburg. Online verfügbar unter 
https://de.statista.com/statistik/daten/studie/156015/umfrage/vorbildung-der-studierenden--in-deutschland-seit-2003/, zuletzt geprüft am 08.12.2018.

Štefanička, Tomáš; Ďuračiová, Renata; Seres, Csaba (2017): Development of a WebBased Indoor Navigation System Using an Accelerometer and Gyroscope: A Case Study at The Faculty of Natural Sciences of Comenius University. In: Slovak Journal of Civil Engineering 25 (4), S. 389. DOI: 10.1515/sjce-2017-0022.

Steiniger, Stefan; Neun, Moritz; Edwardes, Alistair (2006): Foundations of Location Based Servives. Lesson 1. CartouCHe - Lecture Notes on LBS, V. 1.0. Hg. v. University of Zurich. Zürich. Online verfügbar unter https://www.researchgate.net/publication/230777155_Foundations_of_Location_Based_Services, zuletzt aktualisiert am 01.2006, zuletzt geprüft am 09.12.2018.

Stephan, Peter; Frey, Georg; Heck, Ines; Kraus, Peter (2009): Evaluierung von Indoor-Positioning Systems anhand EN ISO 9283 in der SmartFactory. Eine Technologieanalyse unter realitätsnahen Einsatzbedingungen. In: Automation 2009. Der Automatisierungskongress in Deutschland : Kongress Baden-Baden, 16. und 17. Juni 2009. Düsseldorf: VDI Verlag (VDI Berichte, 2067), S. 199-202. Online verfügbar unter https://www.uni-saarland.de/fileadmin/user_upload/Professoren/FreyG/PS_IH_PK_GF_Automation_June_2009.pdf, zuletzt geprüft am 09.12.2018.

Strobel, Christoph (2015): Beacon-Technologie. Das große Ding der kleinen Dinger. Hg. v. CyberForum e.V. Karlsruhe. Online verfügbar unter https://www.techtag.de/it-und-hightech/warum-das-jahr-der-ibeacons-wird/, zuletzt aktualisiert am 09.01.2015, zuletzt geprüft am 09.12.2018.

Sustainable App Developer (Hg.) (2018): QR Code Reader. Google LLC. Online verfügbar unter https://play.google.com/store/apps/details?id=com.qrcodereaderapp, zuletzt aktualisiert am 17.11.2018, zuletzt geprüft am 09.12.2018.

Taentzer, Gabriele (2014): Grundlagen der Anforderungsanalyse. Einführung in die Softwaretechnik. Online verfügbar unter https://www.uni-marburg.de/fb12/arbeitsgruppen/swt/lehre/files/est1415/EST141028.pdf, zuletzt aktualisiert am 28.10.2014, zuletzt geprüft am 08.12.2018. 
Taskin, Deniz (2017): Design of Bluetooth Low Energy based Indoor Positioning System. In: Balkan Journal of Electrical and Computer Engineering, S. 60-65. DOI: 10.17694/bajece.334388.

TERRITORY Embrace (2017): studi.report 2017. Die große Studie zur Situation von Studierenden in Deutschland. Gütersloh. Online verfügbar unter https://www.territory.de/content/uploads/2017/10/studi_report2017.pdf, zuletzt geprüft am 08.12.2017.

Tesche, Yannick (2018): Digitale Nutzung in Deutschland 2018. Abbildung der aktuellen digitalen Mediennutzung in Deutschland und Darstellung möglicher Trends, sowie Analyse des grundsätzlichen Verständnisses von Digitalisierung. Hg. v. Bundesverband Digitale Wirtschaft (BVDW) e.V. Düsseldorf. Online verfügbar unter https://www.bvdw.org/fileadmin/user_upload/BVDW_Marktforschung_Digitale_Nutzung_in_Deutschland_2018.pdf, zuletzt aktualisiert am 02.02.2018, zuletzt geprüft am 08.12.2018.

The PHP Group: SQL Injection. Online verfügbar unter http://php.net/manual/de/security.database.sql-injection.php, zuletzt geprüft am 13.12.2018.

Thomson Reuters (Hg.) (2017): Global Indoor Positioning and Indoor Navigation (IPIN) Market to Grow at a CAGR Of 58.90\% During The Period 2017-2021. Online verfügbar unter https://www.reuters.com/brandfeatures/venture-capital/article?id=4584, zuletzt aktualisiert am 17.04.2017, zuletzt geprüft am 08.12.2018.

Tostmann, Moritz (2016): Tabula rasa im Uni-Viertel: Nur die Fassade steht noch. Ludwigstraße, Ecke Schellingstraße. Hg. v. Abendzeitung Digital GmbH \& Co KG. München. Online verfügbar unter https://www.abendzeitung-muenchen.de/inhalt.ludwigstrasse-ecke-schellingstrasse-tabula-rasa-im-uni-viertel-nur-die-fassade-steht-noch.b02bff08-9ee8-4257-b1a3-0ae3dfe5db1b.html, zuletzt aktualisiert am 09.08.2016, zuletzt geprüft am 08.12.2018.

Tschersich, Markus (2010): Was ist ein mobiles Endgerät? Hg. v. Platingroup GmbH. Stuttgart. Online verfügbar unter https://www.mobile-zeitgeist.com/was-ist-einmobiles-endgeraet/?cookie-state-change $=1531558362220$, zuletzt aktualisiert am 09.03.2010, zuletzt geprüft am 09.12.2018. 
Unger, Ludwig (2016): Baufreigabe für zentralen Bauabschnitt des Philologicum Umfang 33 Millionen Euro - Wissenschaftsminister Dr. Spaenle: "Die Steuergelder sind bestens investiert". Pressemitteilung Nr. 009. Hg. v. Bayerisches Staatsministerium für Unterricht und Kultus. München. Online verfügbar unter https://www.km.bayern.de/pressemitteilung/9921/nr-009-vom-14-01-2016.html, zuletzt aktualisiert am 14.01.2016, zuletzt geprüft am 08.12.2018.

Universität Bern (Hg.) (2018): cUBe. Online-Informationen erkunden. Online verfügbar unter http://www.unibe.ch/universitaet/dienstleistungen/universitaetsbibliothek/ub/cube/index_ger.html, zuletzt geprüft am 08.12.2018.

Universitätsbibliothek Augsburg (Hg.) (2016): QR-Codes für E-Books. Neues Angebot: E-Books auf dem Smartphone. Online verfügbar unter https://www.bibliothek.uni-augsburg.de/bibliothek_nutzen/benfuehrer/nutzung/elektronisch/qrcodes.html, zuletzt aktualisiert am 12.04.2016, zuletzt geprüft am 08.12.2018.

Unni, Ramaprasad; Harmon, Robert (2007): Perceived Effectiveness of Push vs. Pull Mobile Location Based Advertising. In: Journal of Interactive Advertising 7 (2), S. 28-40. DOI: 10.1080/15252019.2007.10722129.

Vatter, André (2011): Foursquare, Facebook Places, Gowalla: Wie Bibliotheken von Location Based Services profitieren können. Hg. v. ZBW - Leibniz-Informationszentrum Wirtschaft. Kiel. Online verfügbar unter https://www.zbw-mediatalk.eu/de/2011/03/foursquare-facebook-places-gowalla-wie-bibliotheken-vonlocation-based-services-profitieren-konnen/, zuletzt aktualisiert am 24.03.2011, zuletzt geprüft am 08.12.2018.

Want, Roy; Hopper, Andy; Falcão, Veronica; Gibbons, Jonathan (1992): The active badge location system. In: ACM Trans. Inf. Syst. 10 (1), S. 91-102. DOI: 10.1145/128756.128759.

Werner, Martin (2014): Indoor Location-Based Services: Prerequisites and Foundations. Place of publication not identified: Springer Science and Business Media. Online verfügbar unter https://www.springer.com/de/book/9783319106984, zuletzt geprüft am 09.12.2018. 
Wikimedia Foundation Inc. (2018a): 2D-Code. San Francisco, CA. Online verfügbar unter https://de.wikipedia.org/w/index.php?oldid=183766564, zuletzt aktualisiert am 23.12.2018, zuletzt geprüft am 24.12.2018.

Wikimedia Foundation Inc. (2018b): EAN-13-5901234123457 - European Article Number. San Francisco, CA. Online verfügbar unter https://de.wikipedia.org/w/index.php?oldid=183585893, zuletzt aktualisiert am 21.12.2018, zuletzt geprüft am 24.12.2018.

Zachár, Gergely; Vakulya, Gergely; Simon, Gyula (2016): Long Distance VLC-based Beaconing for Indoor Localization Applications. Hg. v. University of Pannonia. Ungarn. Online verfügbar unter http://real.mtak.hu/46170/1/IPIN2016.pdf, zuletzt geprüft am 09.12.2018.

Zigbee Alliance (2018): Zigbee 3.0. Online verfügbar unter https://www.zigbee.org/zigbee-for-developers/zigbee-3-0/, zuletzt geprüft am 24.12.2018. 


\section{Abbildungsverzeichnis}

Abbildung 1: Entwurf der Fassade zum Innenhof - Fink Thurner und Cukrowicz Nachbaur.

Abbildung 2: Entwurf der zweigeschossigen Hauptebene mit zentraler Galerieebene

Abbildung 3: Entwurf der zweigeschossigen Hauptebene mit separierten

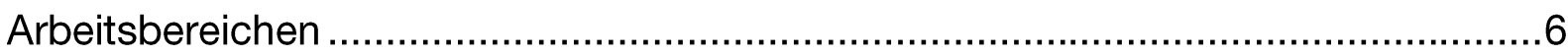

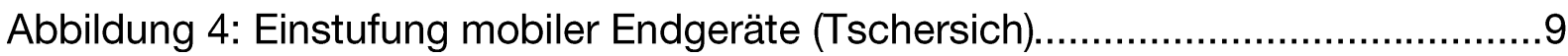

Abbildung 5: LBS Kommunikationsmodell nach Spiekermann ..............................10

Abbildung 6: granulare LBS-Komponenten .................................................11

Abbildung 7: Zusammenspiel und Informationsfluss einzelner LBS-Komponenten .12

Abbildung 8: Absatzentwicklung Smartphones in Deutschland..............................21

Abbildung 9: Absatzentwicklung Smartphones in Deutschland.............................21

Abbildung 10: Smartphone-Verkäufe nach OS ..................................................22

Abbildung 11: Fragmentierung iOS - Stand 31.05.2018 ................................23

Abbildung 12: Fragmentierung Android - Stand 23.07.2018 .............................24

Abbildung 13: Ausschnitt der Architektur von Projekt „Treble“ ..............................25

Abbildung 14: Sensoren der Produktreihe Samsung Galaxy S............................26

Abbildung 15: Hardwarekomponenten für die Standortbestimmung.......................26

Abbildung 16: Überblick über verbaute Sensoren in Smartphones ........................27

Abbildung 17: Überblick über verbaute Sensoren in Smartphones .........................28

Abbildung 18: nicht-funktionale Anforderungen und technische Parameter.............32

Abbildung 19: Techniken zur Positionsbestimmung anhand der Signaleigenschaften

Abbildung 20: Zusammenfassung der betrachteten Techniken.............................38

Abbildung 21: Algorithmen zur Positionsbestimmung ......................................38

Abbildung 22: Klassifikation von Technologien für IPS .......................................41

Abbildung 23: Persona Lisa Huber.................................................................64

Abbildung 24: Persona Krista Vogeler...............................................................66

Abbildung 25: Persona Kevin Lehmann ...........................................................68

Abbildung 26: Persona Louis Momodou...........................................................

Abbildung 27: Strukturelemente eines Textes der Mobile Vision API ......................86 
Abbildung 28: Beispiel „Kunst am Bau“ im Philologicum.....................................87

Abbildung 29: Gesamtarchitektur IPS ......................................................94

Abbildung 30: Bottom-Navigation-Bar der App „Philologicum“ ............................96

Abbildung 31: Farbkonzept UB der LMU ....................................................97

Abbildung 32: Übersicht Neuigkeit und Detailansicht......................................98

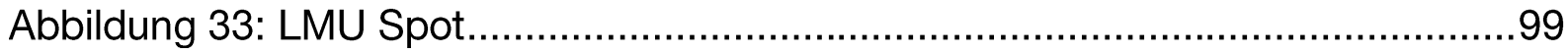

Abbildung 34: Detailansichten LMU Spot ...................................................100

Abbildung 35: Resolving-Workflow für den LBS .............................................101

Abbildung 36: Sitzplatzkapazitäten mit dem System Platzfinder \& Basisinformationen zum Philologicum ............................................................................... 103

Abbildung 37: Einstellungen in normaler Ansicht und in maximalen Kontrast ........104

Abbildung 38: Bildschirme für das Teilen von Inhalten und Notifications ...............105

Abbildung 39: Vereinfachtes Entity-Relation-Ship-Modell der Verknüpfungen.......106

Abbildung 40: Mögliche Attribute der Entität Location .....................................107

Abbildung 41: Workflow Versioncheck .....................................................108

Abbildung 42: Prozessfluss einer Anwendung unter Nutzung von AJAX...............149

Abbildung 43: Beispiel einer JSON-Datei........................................................151

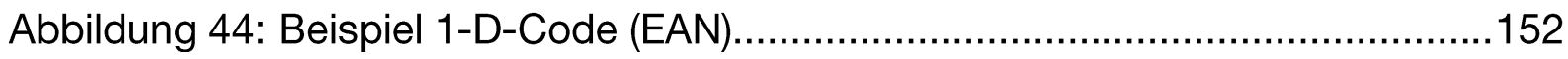

Abbildung 45: Beispiele 2-D-Code (QR-Code, DataMatrix, Aztec-Code) ...............153

Abbildung 46: Beispiel für eine SQL-Injection in Zusammenspiel mit PHP ............155 


\section{Tabellenverzeichnis}

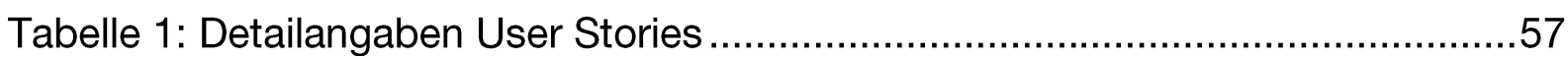

Tabelle 2: Auszug Anforderungskatalog - Frontend ..........................................58

Tabelle 3: Auszug Anforderungskatalog - Indoor Positioning.................................59

Tabelle 4: Auszug Anforderungskatalog - Middleware.........................................60

Tabelle 5: Auszug Anforderungskatalog - Backend..............................................61

Tabelle 6: Auszug Anforderungskatalog - Sonstige..............................................61

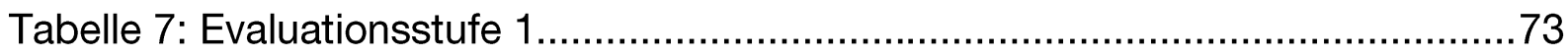

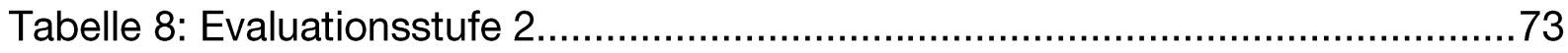

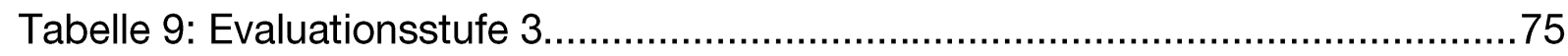

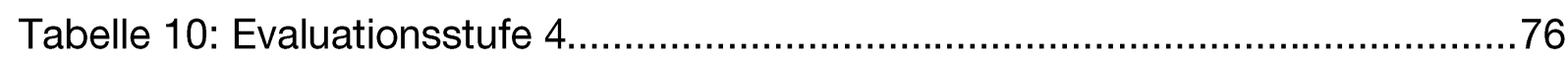

Tabelle 11: Auszug der Messwertanalyse mittels der App „MagneticFingerprint“....77

Tabelle 12: Auszug der Messwertanalyse mittels der App „WLANScanner“ .............77

Tabelle 13: Datenbankschema LMU Linker .....................................................113 


\section{Glossar}

\section{AJAX}

AJAX steht für Asynchronous JavaScript and $\mathbf{X m l}$ und ist ein Konzept der asynchronen Datenübertragung, dass es Webanwendungen ermöglicht neue Daten vom Server zu empfangen oder dem Server für die weitere Verarbeitung zu senden, ohne dass die Seite als Ganzes neu geladen wird. ${ }^{390}$ Die Besonderheit dieser Form der Programmierung ist, dass HTTP-Anfragen durchgeführt werden können, während eine Website angezeigt wird, ohne diese komplett neu zu laden.

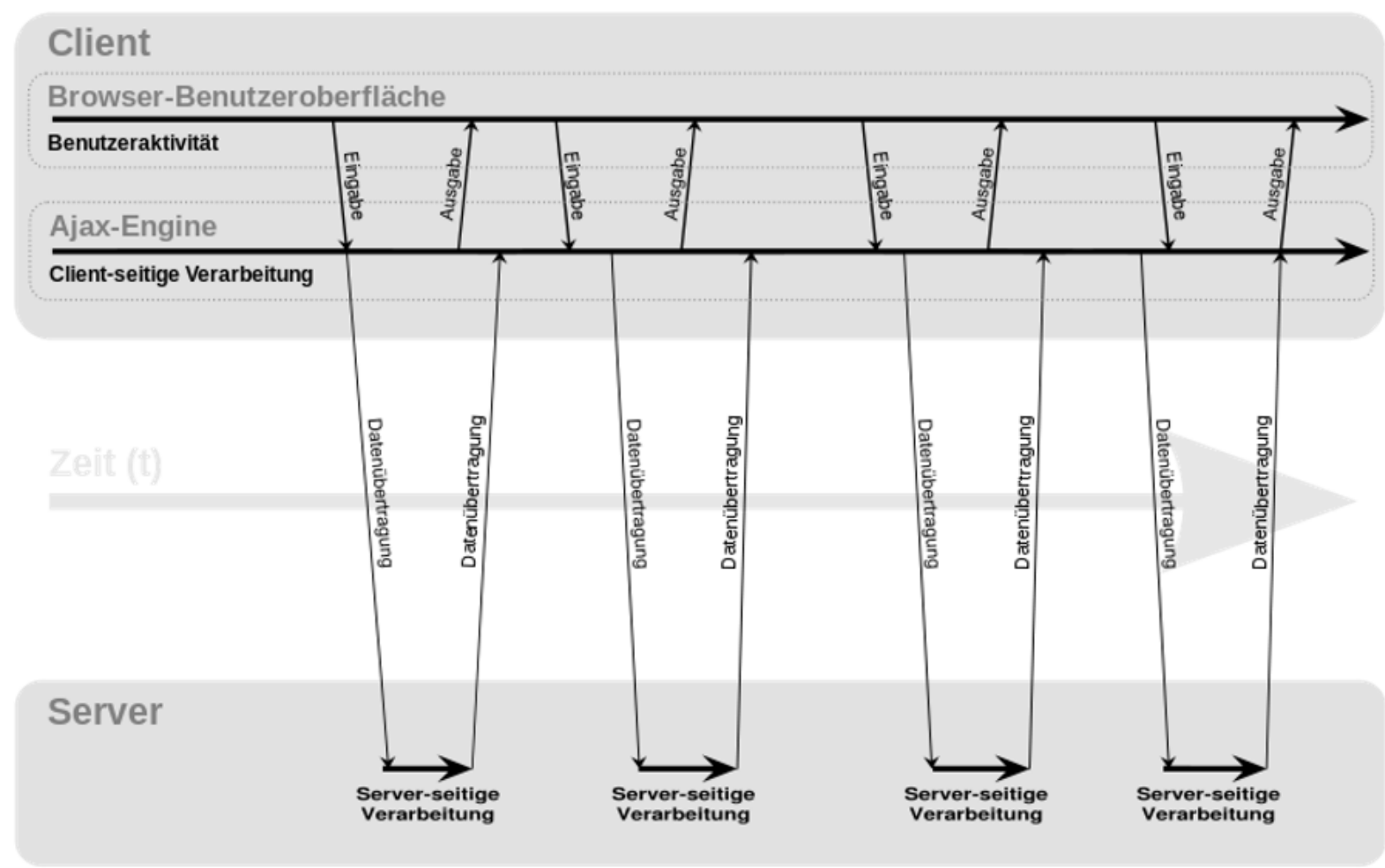

Abbildung 42: Prozessfluss einer Anwendung unter Nutzung von AJAX ${ }^{391}$

Abbildung 42 verdeutlicht die Asynchronität im Vergleich zu einer klassischen Synchronität bei der Kommunikation zwischen Client und Server. Die dargestellt AJAXEngine übernimmt neben der Kommunikation mit dem Server ebenfalls die Anzeige und damit das Rendering der UI. ${ }^{392}$

\footnotetext{
${ }^{390}$ Vgl. SELFHTML e.V. 2018.

${ }^{391}$ Quelle: Garrett 2005.

${ }^{392}$ Vgl. ebd.
} 


\section{Flag}

Eine Flag wird in der Informatik bzw. der Programmierung als ein Indikator für einen bestimmten Status verwendet. Häufig wird dieses Hilfsmittel mit dem Datentyp Boolean umgesetzt, da ein Indikator in der Regel nur den Zustand „An“ oder „Aus“ bzw. „Wahr" oder „Falsch“ annehmen kann. Eine Flag kann gesetzt, gelöscht oder aber überprüft werden, um den aktuellen Zustand abzufragen. Es ist darüber hinaus möglich eine Flag mit mehr als den beiden genannten Zuständen zu belegen. Hierbei ist zu beachten, dass der Datentyp Boolean nicht mehr geeignet ist. Im klassischen Sinne sollte die erst genannte Variante geläufiger sein. Flags finden Verwendung in Kontrollschleifen oder Bedingungsabfragen und repräsentieren zustandsabhängige Entscheidungsmöglichkeiten.

\section{Gradle}

Gradle basiert auf JAVA und ist, wie Apache Mave oder ANT, ein Build-ManagementAutomatisierungs-Tool, dessen Hauptaufgabe es ist, den Erstellungsprozess eines Anwendungsprogrammes, in Form von Code-Kompilierung zu realisieren. ${ }^{393}$ Gradle übernimmt somit alle für die Erstellung einer Anwendung notwendigen Schritte und prüft beispielsweise die Einhaltung aller Abhängigkeiten. Im Rahmen der I/O-Konferenz im Jahr 2013 hat Google bekannt gegeben, zukünftig für seine DIE Android Studio Gradle zu nutzen, da diese unter anderem gut an die Android Gegebenheiten anzupassen gewesen sei. ${ }^{394}$

\section{InnoDB}

InnoDB ist die Database Engine oder auch Storage Engine für das Datenbankmanagementsystem MySQL. ${ }^{395}$ InnoDB bietet transaktionssichere Lese- und Schreibzugriffe, d. h. es bietet Begin-, Commit- und Rollback-Funktionen. Dadurch wird sichergestellt, dass eine Abfrage oder ein Satz zusammengehöriger Abfragen entweder ganz oder gar nicht, auf keinen Fall jedoch unvollständig ausgeführt wird. ${ }^{396}$

\footnotetext{
${ }^{393}$ Vgl. Gradle Inc. 2018.

${ }^{394}$ Vgl. Schmidt 2013.

${ }^{395}$ Vgl. Oracle 2018.

${ }^{396}$ Ebd.
} 


\section{JSON}

JavaScript Object Notation oder kurz JSON ,ist ein schlankes Datenaustauschformat, das für Menschen einfach zu lesen und zu schreiben und für Maschinen einfach zu parsen (Analysieren von Datenstrukturen) und zu generieren ist. ${ }^{‘ 397}$ Syntaktisch besteht das Austauschformat aus einer spezifischen Anordnung aus Klammern, Doppelpunkten und Kommas. ${ }^{398}$ Als Textformat ist es unabhängig von Programmiersprachen und somit prädestiniert für eine enorm hohe Interoperabilität.

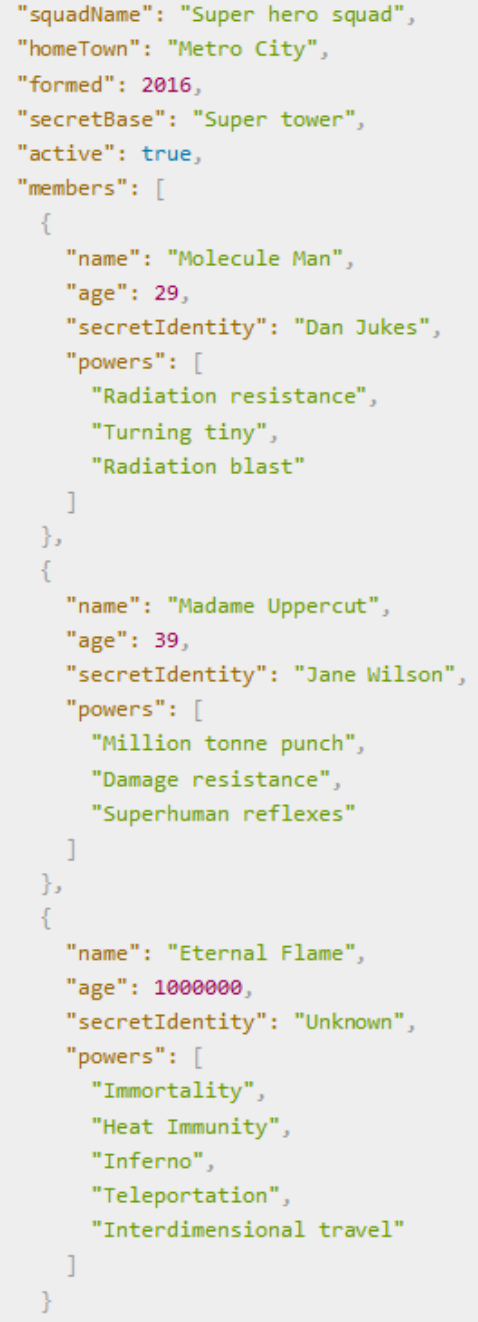

Abbildung 43: Beispiel einer JSON-Datei ${ }^{399}$

\footnotetext{
${ }^{397}$ Crockford.

${ }^{398} \mathrm{Vgl}$. ECMA International 2017, S. 3.

${ }^{399}$ Quelle: Massmann 2018.
} 
JSON baut grundsätzlich auf zwei Strukturen auf. Zum einen sind dies Namen-WertPaare und zum anderen geordnete Listen von Werten. ${ }^{400}$ Als textbasiertes Datenformat ist es an die JavaScript Object Syntax angelehnt, damit können unterschiedlichste Datentypen wie Strings, Zahlen, Arrays, Booleans oder andere Objekttypen verwendet werden. ${ }^{401}$ Abbildung 43 zeigt ein Beispiel für eine JSON-Datei mit unterschiedlichen Paaren aus Namen und Werten sowie Arrays. So ist beispielsweise „members“ ein Array mit insgesamt 3 Felden, zu erkennen an den eckigen Klammern. Die einzelnen Felder wiederrum sind durch einfache Kommata voneinander getrennt. Anhand von „powers“ wird gezeigt, dass auch multidimensionale Arrays mit JSON einfach abbildbar sind. Wichtig ist anzumerken, dass JSON immer doppelte Hochkommata benötigt. Einfache Hochkommata sind unzulässig. Abschließend sei noch zu nennen, dass JSON ein reines Datenformat ist und dementsprechend nur Eigenschaften, aber keine Methoden bereitstellt. ${ }^{402}$

\section{Optoelektronisch lesbare Codes}

Optoelektronisch lesbare Codes lassen sich in 1-D- und 2-D-Codes unterteilen. 1-DCodes sind klassische Strichcodes, also eine Abfolge von unterschiedlich breiten Strichen und Lücken, teilweise ergänzt durch Zeichenreihen. ${ }^{403}$ Die Strichfolgen sind immer in einer einzelnen Reihe angeordnet, man spricht deswegen von eindimensionalen Code. Bekannte Strichcodearten sind Handelsstrichcodes wie die European Article Number, kurz EAN, oder auch ISBN und ISSN, die wiederum im EAN-13-Format codiert werden.

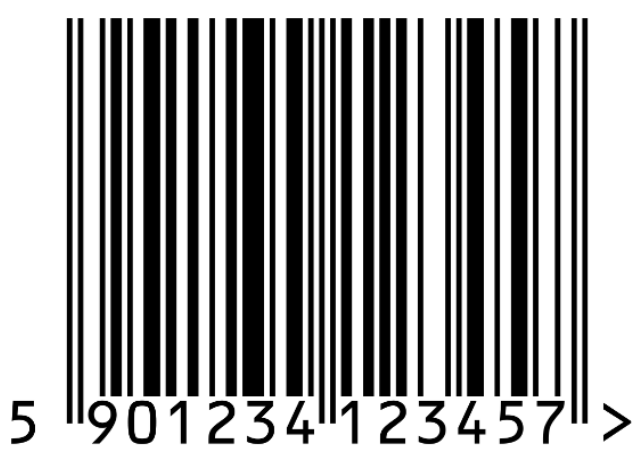

Abbildung 44: Beispiel 1-D-Code (EAN) ${ }^{404}$

\footnotetext{
${ }^{400}$ Vgl. Crockford

${ }^{401}$ Vgl. Massmann 2018.

${ }^{402}$ Vgl. Massmann 2018.

${ }^{403}$ Vgl. identWERK GmbH.

${ }^{404}$ Quelle: Wikimedia Foundation Inc. 2018b.
} 
2-D-Codes sind hingegen rechteckige Symbole, die sich hauptsächlich aus kleinen Quadraten, Strichen oder Punkten zusammensetzen. ${ }^{405}$ Es wird versucht in den Lücken einen möglichst maximalen Kontrast, für eine erhöhte Erkennungsrate zu erreichen. Die Daten sind im Vergleich zum Strichcode nicht nur in eine Richtung codiert, sondern in Form einer Fläche über zwei Dimensionen. Beispiele für solche Codierungen sind gestapelte Codes, wie Codablock oder Code 49 sowie Matrix-Codes, wie QR-Codes, DataMatrix oder Aztec-Code. Alle Formen der Codierungen können von einem optischen Lesegerät, wie Barcodelesegeräten oder Kameras maschinell eingelesen und weiterverarbeitet werden.

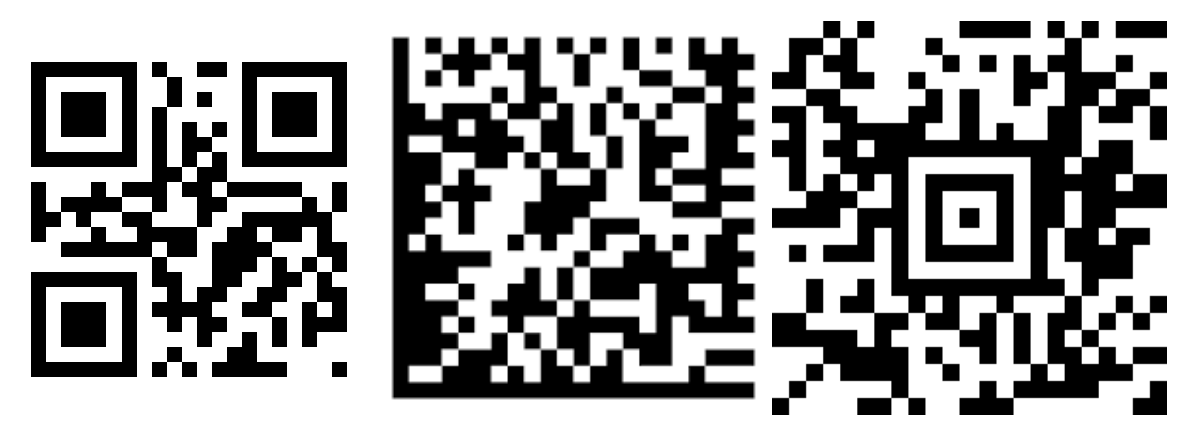

Abbildung 45: Beispiele 2-D-Code (QR-Code, DataMatrix, Aztec-Code) $)^{406}$

\section{Phablet}

Bei einem Phablet handelt es sich um ein Hybridgerät aus Tablet und Smartphone. Aus dieser Symbiose ergibt sich die Wortschöpfung „Phablet“, abgeleitet aus „Phone“ und „Tablet“. In der Regel handelt es sich um Smartphones mit einer Bildschirmdiagonale zwischen fünf und sieben Zoll. ${ }^{407}$ Hatten Geräte dieser Klasse in der Vergangenheit noch teilweise unhandliche Maße, so sind Phablets in der heutigen Zeit, aufgrund nahezu randloser Displays, beinahe genauso ergonomisch, wie Smartphones unter fünf Zoll. Darüber hinaus haben Phablets meist softwareseitig spezielle Hilfsprogramme implementiert, die eine erleichterte Bedienung ermöglichen.

\section{Störfaktoren}

Funkfrequenzen entstehen mittels elektrischer sowie magnetischer Felder und dienen der Übertragung von Daten oder der Kommunikation. ${ }^{408}$ Aufgrund ihrer physikalischen

\footnotetext{
${ }^{405}$ Vgl. identWERK GmbH.

${ }^{406}$ Quelle: Wikimedia Foundation Inc. 2018a.

${ }^{407}$ Vgl. Dübbel 2015.

${ }^{408}$ Vgl. Schnabel.
} 
Eigenschaften können elektromagnetische Funkfrequenzen bei einer freien Ausbreitung im Raum potentiellen Störfaktoren unterliegen.

Die Reflexion tritt auf, wenn eine elektromagnetische Frequenz auf ein Objekt, wie beispielsweise eine Wand eines Raumes trifft. Die einfallende Welle teilt sich dann in einen reflektierten Anteil und einen Anteil, der in das Material eindringt und entsprechend absorbiert bzw. gedämpft wird. ${ }^{409}$ Je nach Beschaffenheit des Materials können die beiden Anteile prozentual voneinander abweichen. Darüber hinaus ist es möglich, dass das Signal gänzlich zurückgeworfen wird. Gerade bei rauen Oberflächen steigt die Wahrscheinlichkeit, dass die Funkwellen nicht nur reflektiert, sondern gar in alle Richtungen zerstreut werden. ${ }^{410}$

Als Beugung bezeichnet man die Ablenkung einer elektromagnetischen Welle an einem Hindernis. ${ }^{411}$ Die Feldstärke des Signales nimmt, aufgrund der Dämpfung rasch ab. Darüber hinaus werden Funkwellen mit zunehmender Reichweite immer schwächer. Prinzipiell gilt, je höher die Reichweite desto anfälliger ist das Funksignal für Störungen. Sollten sich die Signale überlagern können Interferenzen entstehen. Die sich überlagernden Wellen können sich im positiven Fall verstärken, oder im negativen Fall gegenseitig auslöschen. ${ }^{412}$

\section{SQL-Injection}

Eine SQL-Injection (engl. für Einschleusung, Injektion oder Einspritzung) ist das Ausnutzen einer Sicherheitslücke im Zusammenhang mit SQL-Datenbanken. Der Angreifer versucht über die Anwendung eigene Datenbankbefehle einzuschleusen und somit die Datenbank unbefugt zu manipulieren. Verhindert werden kann dieser Angriffsvektor, durch Maskieren oder Escapen, allgemein gesagt durch eine solide Validierung von Nutzereingaben auf bestimmte Muster und Vorgaben hin. Eine weitere Maßnahme zum Absichern ist es die Datenbankverbindung nicht als Nutzer mit Administrationsrechten aufzubauen, sondern ein gesondertes Nutzerkonto mit verringerten Rechten zu nutzen. ${ }^{413}$

\footnotetext{
${ }^{409}$ Vgl. Küng 2009, S. 10.

${ }^{410}$ Vgl. ebd., S. 15.

${ }^{411}$ Vgl. Joachim Herz Stiftung.

${ }^{412} \mathrm{Vgl}$. ebd.

${ }^{413}$ Vgl. The PHP Group.
} 


\section{MySQL \& PHP Code:}

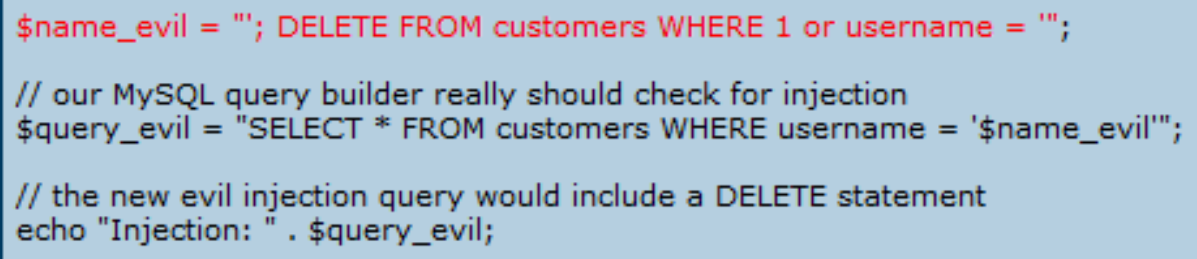

Display:

SELECT $*$ FROM customers WHERE username $={ }^{\prime}$ '; DELETE FROM customers WHERE 1 or username $={ }^{\prime}$ '

Abbildung 46: Beispiel für eine SQL-Injection in Zusammenspiel mit PHP ${ }^{414}$

\section{Ultra Wideband}

Ultra Wideband ist ein Breitbandsignal mit einer Bandbreite von mehr als $500 \mathrm{Mhz}$ Bandbreite im Frequenzbereich zwischen 3,1 Ghz und 10,6 Ghz. Die UWB-Technik ist eine Technik für WLANs, die höhere Übertragungsgeschwindigkeiten bietet als die Spezifikation $802.11 .{ }^{415}$

\section{Wearables}

Der Begriff steht für kleine, vernetzte Computer, die am Körper getragen werden und den Nutzer bei der Bewältigung seines Alltages unterstützen sollen. ${ }^{416}$ Wearables finden sich vor allem in den Bereichen Gesundheit, Lifestyle und Fitness. Beispiele für diese Geräteklasse sind Smartwatches, Fitnesstracker und digitale Brillen. „WearableComputing" ist die tangierende Disziplin in der Informatik und beschäftigt sich unter anderem mit spezifischen Gesichtspunkten dieser speziellen Form der Mensch-Maschine-Interaktion. ${ }^{417}$ Begünstigt wird der Trend zu Wearables durch hochentwickelte Sensorik auf kleinsten Raum, die zu einer Miniaturisierung der Komponenten führt sowie durch eine permanente Verarbeitung von Daten. ${ }^{418}$

\footnotetext{
${ }^{414}$ Quelle: mysql - sql injection prevention.

${ }^{415} \mathrm{Vgl}$. DATACOM Buchverlag GmbH 2013.

${ }^{416}$ Vgl. Hery-Moßmann 2017.

${ }^{417}$ Bendel.

${ }^{418}$ Vgl. ebd.
} 


\section{Zigbee}

Zigbee ist eine Spezifikation für drahtlose Netzwerke mit geringem Datenaufkommen und wird überwiegend für beispielsweise Hausautomation genutzt. Die Distanz für die Kommunikation im Netzwerk zeichnet sich durch eine kurze Reichweite bis etwa 100 Meter aus. ${ }^{419}$

${ }^{419}$ Vgl. Zigbee Alliance 2018. 


\section{Eigenständigkeitserklärung}

Hiermit versichere ich, dass ich die vorliegende Arbeit selbstständig und ohne Benutzung anderer als der angegebenen Quellen und Hilfsmittel angefertigt habe. Alle Stellen, die wörtlich oder sinngemäß veröffentlichten oder nicht veröffentlichten Quellen entnommen wurden, sind als solche kenntlich gemacht.

Kai Barth

München, den 02.01.2019 